(1) GenHotel-EA3886, Evry-Paris VII Universités, Evry-Genopole ; (2) Faculté de Médicine, Université de Coimbra, Portugal ; (3) CHSF, Corbeil-Essonnes ; (4) Service de Rhumatologie, Hôpital Lariboisière, Paris ; (5) Unité de Génétique Clinique, Hôpital Lariboisière, Paris

Introduction : La Polyarthrite Rhumatoïde (PR) est une maladie inflammatoire chronique caractérisée par l'inflammation persistante du tissu synovial conduisant à la destruction des articulations affectées. L'apoptose insuffisante des macrophages, des fibroblastes et des lymphocytes synoviaux est un mécanisme qui pourrait contribuer à la persistance de la maladie. Les caspases sont des acteurs importants de l'initiation (initiateurs) et du déroulement (effecteurs) de la mort cellulaire programmée. La caspase 7 est un effecteur qui possède quatre variants de transcription codant pour trois isoformes distinctes (bêta, alpha et delta). L' isoforme bêta de la caspase 7 est dépourvue de site actif et peut agir en tant qu'inhibiteur dominant des isoformes actives alpha et delta de la procaspase 7 . Une étude cas/contrôles dans une population caucasienne espagnole a montré une association significative du génotype GG du polymorphisme rs2227309 $\mathrm{A}>\mathrm{G}$ du gène $C A S P 7$ avec la $\mathrm{PR}$. Le but de notre travail est d'étudier l'association et la liaison de ce polymorphisme rs2227309 avec la PR dans des familles trio caucasiennes françaises (un patient atteint et ses 2 parents). De plus, le niveau de l'expression de l'ARNm des isoformes bêta et alpha de la CASP7 est évalué chez 24 patients atteints de PR et chez 15 contrôles caucasiens français. Patients et Méthodes. Le polymorphisme rs2227309 du gène CASP7 est génotypé dans un échantillon de 100 familles trio. Une réplication dans un deuxième échantillon de 100 familles trio a été réalisée. Le génotypage a été effectué par PCR avec sondes fluorescentes (TaqMan). L'information d'association et de liaison est obtenue par le test de déséquilibre de transmission et le risque relatif de génotype. L'expression relative de l'ARNm des isoformes bêta et alpha de la CASP7 a été quantifiée dans les cellules sanguines périphériques de 24 patients atteints de PR et de 15 contrôles par transcription inverse à partir de l'ARN total puis par PCR quantitative. Résultats. Nous avons observé une transmission significativement plus élevée du génotype AA chez les patients avec facteur rhumatoïde que chez les contrôles $(P=0.04)$. La réplication dans un second échantillon de familles n'a pas reproduit ce résultat. L'analyse combinée des deux échantillons testés ne présente donc aucun résultat d'association entre le SNP testé et la maladie. Les isoformes beta et alpha de CASP7 sont moins exprimées chez les patients que chez les contrôles $(P=0.07, P=0.0006$, respectivement). Discussion : Les résultats de l'étude d'association nous permettent de conclure à l'absence d'implication du polymorphisme rs2227309 du gène CASP7 dans la composante génétique de la $\mathrm{PR}$ pour la population testée. Le locus du gène CASP7 n'est pas représenté parmi les 19 régions liées à la maladie dans la population française caucasienne. De plus, la dernière étude du génome entier par l'analyse de SNPs dans une population Caucasienne (UK) a permis de mettre en évidence de nouveaux loci associés à la PR, mais aucun ne contient la région du gène CASP7. Les données d'expression ont permis de mettre en évidence une baisse significative de l'expression de l'isoforme alpha de la caspase 7 entre les contrôles et les patients PR. Cela suggère que l'activité apoptotique médiée par la caspase 7 au niveau des cellules du sang périphérique est moins importante chez les malades par rapport aux témoins. L'expression de l'isoforme beta est également moins élevée chez les patients, mais de manière non significative. Donc son activité inhibitrice des formes actives des caspases peut également conduire à une baisse de l'activité apoptotique chez les patients PR. Conclusion. Le polymorphisme rs2227309 du gène CASP7 n'est pas associé à la PR dans la population française caucasienne. Des investigations complémentaires de la régulation de l'expression du gène CASP7 sont nécessaires pour confirmer son implication dans les mécanismes apoptotiques impliqués dans cette maladie.

Mots-clés : CASP7, polyarthrite rhumatoïde, expression.

\section{Génétique Moléculaire}

\section{- 305 /2. CONTRIBUTION OF BRCA1 AND BRCA2 MUTATIONS TO BREAST CANCER IN TUNISIA}

W. Troudi (1, 2), N. Uhrhammer (3), C. Sibille (4), W. Mahfoudh (5), T. Jalabert (3), L. Chouchane (5), Y.J. Bignon (3), F. Ben Ayed (2), A. BenAmmar Elgaaied (1)

(1) Laboratory of Genetics, Immunology and Human Pathology at the Faculty of Sciences of Tunis. Faculty of Sciences of Tunis. University El Manar I; (2) Salah Azaiez Institute of Carcinology of Tunis ; (3) Laboratoire Diagnostic Génétique et Moléculaire. Centre Jean-Perrin. Clermont-Ferrand, France ; (4) Laboratory of Molecular Genetic of Hereditary Pathologies. Center of Human Genetics UCL. Bruxelles; (5)
Laboratory of Molecular Immuno-Oncology, Faculty of Medecine of Monastir. Tunisia

Hereditary breast cancer accounts for 3-8 \% of all breast cancers. Combination of $B R C A I$ and $B R C A 2$ genes mutations is responsible for $30 \%$ of hereditary breast cancer cases. To investigate the prevalence of BRCAI and $B R C A 2$ gene mutations in familial breast cancer patients with affected relatives in Tunisia, 36 patients who had at least one first degree relative affected with breast and/or ovarian cancer were analysed. Thirty three patients were suggestive of $B R C A I$ mutation and 3 were suggestive of $B R C A 2$ mutation. Four mutations in BRCAI gene were detected among which, one novel frame-shift mutation (c.211dupA) described in two unrelated patients and 3 frameshift mutations ; c.4041delAG, c.2551delG and c.5266dupC. In this study we describe the c.5266dupC mutation which was described only among Jewish Ashkenazi population. Two frameshift mutations (c.1309delAAAG and c.5682ins A) were observed in BRCA2 gene. Nineteen percent $(7 / 36)$ of the familial cases were altered on BRCAI or $B R C A 2$ genes with deleterious mutations. Almost all the cases mutated by deleterious mutations on $B R C A l$ gene reported a family history of breast and/or ovarian cancer in the index case or in their relatives. Our data are the first to contribute to information on mutation spectrum of BRCA genes in Tunisia and offer a recommendation for clinical genetic testing policy.

Mots-clés : $B R C A 1, B R C A 2$, mutation analysis breast cancer, tunisia.

\section{aP306 / 6. PARTICULARITÉS PROTÉIQUES ET MOLÉCU- LAIRES D'UNE COHORTE DE 69 PATIENTS ÉVOQUANT UNE} SARCOGLYCANOPATHIE

M. Trabelsi (1), N. Kavian (1), V. Commere (1), N. Deburgrave (1), C. Beugnet (1), S. Llens (1), J.C. Barbot (1), J.C. Kaplan (1-3), F. Leturcq (1-3), J. Chelly (1-3)

(1) Laboratoire de Biochimie et Génétique Moléculaire. Hôpital Cochin, Paris, France; (2) Institut Cochin, Université Paris Descartes, CNRS (UMR 8104), Paris, France ; (3) Inserm, U567, Paris, France

Notre étude a consisté à définir le spectre de mutations au niveau des gènes alpha-, beta-, gamma-, delta-Sarcoglycanes (SGCA, SGCB, SGCG et $S G C D$ ) chez des patients évoquant cliniquement et biologiquement une sarcoglycanopathie (myopathie des ceintures à transmission autosomique récessive, LGMD type 2C-F). Après avoir exclu les patients présentant les trois mutations les plus fréquentes, en France, à savoir la c.229C $>\mathrm{T}(\mathrm{R} 77 \mathrm{C})$ du (SGCA), la c.525delT et la c. $848 \mathrm{G}>\mathrm{A}(\mathrm{C} 283 \mathrm{Y})$ du (SGCG), nous avons étudié une cohorte de 69 patients. La biopsie musculaire et l'étude par Western-Blot des protéines musculaires n'ont été réalisées que chez 48 patients révélant des anomalies qualitatives des alpha- et gamma-sarcoglycanes (SG). La recherche de mutations a été basée sur le séquençage des gènes alpha-, beta-, gamma-, delta-Sarcoglycanes et l'étude par PCR semiquantitative fluorescente (QF-PCR) des SGCA et SGCG. Le diagnostic a été confirmé chez $75 \%$ (36/48) des patients présentant une anomalie qualitative des a- et g-s (SG) alors qu'il n'a été établi que chez $52 \%(11 / 21)$ des patients n'ayant pas eu de biopsie musculaire. Le SGCA était muté dans $55.3 \%$ des cas alors que le $S G C G$ et $S G C B$ l'étaient dans 25.5 et $17 \%$ des cas, respectivement. Au niveau du $S G C D$, une seule mutation hétérozygote a été mise en évidence chez un patient double hétérozygote pour le $S G C B$ et le $S G C D$. Parmi les 43 mutations identifiées dans notre cohorte, 22 ont été décrites pour la première fois. Concernant les patients restants, dont 12 avec un profil Western Blot anormal, une seule mutation a été identifiée dans $45.5 \%$ des cas (10/22) alors qu'aucune mutation n'a été mise en évidence dans $54.5 \%(12 / 22)$ des cas. Ces résultats, associés à l'exclusion des quatre gènes des sarcoglycanes par génotypage utilisant des marqueurs microsatellites polymorphes, évoquent l'implication, dans ce phénotype sarcoglycane-like, d'autres gènes codant pour des protéines interagissant avec les SG. Les résultats de notre travail nous ont permis de révéler l'importance du Western-Blot dans la stratégie diagnostic des sarcoglycanopathies, la fréquence élevée des délétions d'exons $(15.8 \%)$ au niveau des $S G C A$ et $S G C G$ ainsi que la présence d'une région hot spot de duplications au niveau de l'exon 1 du $S G C B(5 / 69)$.

Mots-clés : Sarcoglycanopathies Western Blot séquençage.

שP307 / 17. DÉFAUT DE LOCALISATION NUCLÉAIRE DE LA PROTÉINE CDKL5 DÛ À DES MUTATIONS FAUX-SENS DU DOMAINE KINASE DE CDKL5 CHEZ DES PATIENTES PRÉSENTANT UNE ENCÉPHALOPATHIE CONVULSIVANTE SÉVÈRE

H. Rosas-Vargas (1), N. Bahi-Buisson (2), C. Philippe (3), B. Girard (4), J. Nectoux $(1,4,5)$, M. Ange N'Guyen (6), C. Gitiaux (2), P. Jonveaux (3), J. Chelly $(1,4,5)$, T. Bienvenu $(1,4,5)$

(1) Institut Cochin, Universite Paris Descartes, CNRS UMR (8104), Paris, France; (2) Department of Neuropediatrics, Necker-Enfants-Malades 
Hospital, Paris, France; (3) Laboratoire de Génétique, EA3441, CHU Brabois, Vandauvre-Les-Nancy, France ; (4) Assistance Publique-Hôpitaux de Paris, Hôpital Cochin, Laboratoire de Biochimie et Genetique Moleculaire, Paris, France ; (5) Inserm, U567, Paris, France ; (6)Service de Pédiatrie, CHU Grenoble, Grenoble, France

Des mutations dans la séquence codante du gène $C D K L 5$ sont à l'origine de spasmes infantiles, d'épilepsies sévères pharmacorésistantes et de formes atypiques de syndrome de Rett. Actuellement une vingtaine de mutations distinctes ont été identifiées à travers le monde. Aucune corrélation claire génotype-phénotype n'a encore été établie. Nous avons examiné par chromatographie liquide haute pression en condition dénaturante et par séquençage direct la totalité de la région codante du gène $C D K L 5$ chez 151 filles présentant soit une encéphalopathie convulsivante sévère inexpliquée, soit un syndrome de Rett atypique en absence de mutations dans le gène $M E C P 2$. Nous avons ainsi identifié trois nouvelles mutations faux-sens situées dans le domaine catalytique kinase de $C D K L 5$ (p.Ala40Val, p.Arg65Gln, p.Leu220Pro). L'analyse de ségrégation familiale a montré que la variation $\mathrm{p}$.Arg65Gln était présente chez le père asymptomatique, suggérant que cette variation est un polymorphisme non délétère. Toutefois, les deux autres mutations faux-sens p.Ala40Val et p.Leu220Pro étaient de novo. La mutation p.Ala40Val a été identifiée chez deux patientes non apparentées et représente la première mutation récurrente du gène $C D K L 5$. Pour les deux mutations de novo, nous avons effectué une mutagénèse dirigée du gène $C D K L 5$ à partir de l'ADNc de $C D K L 5$ cloné dans un vecteur plasmidique permettant l'expression d'une protéine de fusion CDKL5-GFP. Après transfection des différentes constructions sauvage et mutées dans des cellules COS-7, nous avons montré que ces mutations faux-sens entraînent une localisation cytoplasmique de la protéine CDKL5 mutée, alors que la protéine CDKL5 sauvage présente une localisation essentiellement nucléaire. Ainsi, une anomalie de localisation subcellulaire de la protéine CDKL 5 est associée à la survenue d'un retard sévère du développement, avec hypotonie sévère, spasmes infantiles, stéréotypies manuelles, et épilepsie précoce.

Mots-clés : CDKL5, MECP2, épilepsie.

IP308 / 18. L'EFFET PROTECTEUR DU POLYMORPHISME P.VAL66MET DU FACTEUR NEUROTROPHIQUE BDNF (BRAIN. DERIVED NEUROTROPHIC FACTOR) SUR LA SURVENUE DES CRISES D'ÉPILEPSIE DANS LE SYNDROME DE RETT

J. Nectoux (1, 2), N. Bahi-Buisson (3), I. Guellec (3), J. Coste (4), N. De Roux (5), H. Rosas-Vargas (1, 2), M. Tardieu (6), J. Chelly (1, 2, 7), T. Bienvenu $(1,2,7)$

(1) Institut Cochin, Université Paris-Descartes, CNRS (UMR 8104), Paris, France; (2) Inserm U567, Paris, France; (3) Service de Neuropédiatrie, Hôpital Necker-Enfants Malades, AP-HP, Paris, France; (4) Service de Biostatistiques et d'Informatique Médicale, AP-HP, Hôpital Cochin, Paris, France; (5) Service de Biochimie et Génétique Moléculaire, Hôpital Robert-Debré, Paris, France; (6) Service de Neurologie, CHU Bicêtre, Le Kremlin-Bicêtre, France; (7) Laboratoire de Biochimie et Genetique Moleculaire, Hôpital Cochin, Paris, France

Les mutations du gène $M E C P 2$ sont à l'origine des formes classiques et atypiques du syndrome de Rett (RTT) qui touchent essentiellement les filles. Chez la fille RTT, deux facteurs génétiques sont susceptibles d'influencer le phénotype : l'inactivation du chromosome X (XCI) et le type et la position des mutations $M E C P 2$. Cependant, l'XCI (étudiée sur les lymphocytes) et le génotype MECP2 ne permettent pas d'expliquer clairement les variations phénotypiques chez les patientes RTT suggérant la participation d'autres facteurs environnementaux et génétiques. Le facteur neurotrophique BDNF, essentiel à la survie neuronale, à la différenciation, et à la plasticité synaptique, est l'une des cibles de MeCP2. BDNF présente un polymorphisme fonctionnel p.Val66M et, affectant le trafic intracellulaire et la sécrétion de BDNF. Notre travail avait pour objectif de mettre en évidence une association entre le polymorphisme p.Val66M et (c.G196A) dans BDNF et la sévérité de la symptomatologie dans le RTT en tenant compte du génotype MECP2. 81 patientes RTT présentant une mutation $M E C P 2$ ont été inclues. Dans ce travail, nous avons mis en évidence une relation entre le génotype MECP2 et certains critères cliniques, comme la sévérité des troubles moteurs, la précocité et la sévérité des crises d'épilepsie. Les mutations faux-sens, essentiellement celles localisées dans le domaine MBD, sont ainsi associées à une plus grande sévérité des crises d'épilepsie $(\mathrm{p}=0.003)$, et les mutations non-sens à une plus grande sévérité des atteintes moteurs $(p=0.05)$. Aucune différence significative dans la distribution du polymorphisme p.Val66M et n'a été observée en fonction de la sévérité des troubles moteurs, de la communication verbale et non verbale, des stéréotypies gestuelles, de la dyspraxie
Toutefois, les filles RTT présentant le génotype p.Val66Val présentent des crises d'épilepsie plus précocement (avant 2 ans) que les filles RTT porteuses de l'allèle Met. Dans notre étude, aucune patiente présentant une crise d'épilepsie précoce ne présentait l'allèle Met, suggérant que l'allèle Met présente un effet protecteur sur la survenue précoce des crises d'épilepsie dans le syndrome de Rett.

Mots-clés : BDNF, MECP2, Rett syndrome.

-P309/21. MORT SUBITE DU NOURRISSON ET SYNDROME DU QT LONG CONGÉNITAL

G. Millat (1), B. Kugener (2), P. Chevalier (3), H. Huang (4), M. Chahine (4), D. Malicier (5), R. Rousson (1), C. Rodriguez-Lafrasse (1)

Laboratoire de Cardiogénétique Moléculaire, Centre de Biologie Est, Hospices Civils de Lyon ; (2) Urgences Nourissons, Hôpital Debrousse, Lyon ; (3) Unité de Cardiologie et Soins Intensifs, Hôpital CardioVasculaire et Pneumologique L-Pradel, Bron ; (4) Centre de recherche Université Laval Robert-Giffard, Québec, Canada ; (5) Institut Médico-légal, Domaine Rockefeller, Lyon, France

La définition de la Mort Subite du Nourrisson est le décès brutal, inattendu, d'un nourrisson âgé de moins de 1 an, apparemment en bonne santé, pendant son sommeil. Malgré des mesures de prévention d'ordre comportemental (couchage des bébés sur le dos sans couette ni oreiller, limitation du tabagisme passif,...), la Mort Subite du Nourrisson reste la première cause de décès au cours de la $\mathrm{I}^{\mathrm{re}}$ année de vie. En plus de facteurs de risque environnementaux, différents facteurs de risques biologiques, y compris génétiques, ont également été décrits. Quelque soit le gène suspecté, la composante génétique peut s'envisager de 2 façons : existence de mutations qui par elles-mêmes suffisent à l'expliquer le décès ou présence de polymorphismes qui peuvent prédisposer certains nourrissons aux décès dans des conditions critiques (fièvre, infection...). De précédentes études ont ainsi permis de démontrer que des variants exoniques (mutations et/ou polymorphismes) de gènes impliqués dans le syndrome du QT Long pouvaient expliquer certains cas de Mort Subite Inexpliquée du Nourrisson. Le syndrome du QT long congénital est une pathologie cardiaque cliniquement et génétiquement hétérogène caractérisée par un allongement de l'espace QT à l'ECG. Cet allongement est associé à un risque élevé de survenue de troubles graves du rythme ventriculaire (torsades de pointes, fibrillation ventriculaire) pouvant entraîner syncope et mort subite. Environ $75 \%$ des cas de syndrome du QT Long sont dues à des mutations affectant des gènes codant des sous unités de canaux sodiques et potassiques [KCNQ1, KCNH2, SCN5A, KCNE1 et KCNE2]. Afin de mieux préciser le lien entre la Mort Subite du Nourrisson et le Syndrome du QT long, une cohorte de 55 nourrissons décédés de façon inexpliquée avant 1 an a été étudiée en simple aveugle. Pour chacun des cas, il a été réalisé d'une part un protocole d'autopsie standardisé (radiographies, toxicologie, neuropathologie, histologie, microbiologie, virologie,...) et d'autre part une étude moléculaire des gènes $K C N Q 1, K C N H 2, S C N 5 A, K C N E 1$ et $K C N E 2$. Le profil moléculaire établi pour chaque cas contient non seulement la ou les mutations probables, mais également les polymorphismes préalablement décrits dans la littérature comme ayant un effet sur l'intervalle QT. Le protocole d'autopsie standardisé a pu expliquer 19 des 55 décès. Pour ces 19 cas, aucune mutation n'a été identifiée sur les gènes étudiés. Inversement, pour les 36 décès qui demeuraient sans diagnostic après l'autopsie, 6 sont porteurs de mutations affectant les gènes du syndrome du QT long. Tous les mutants identifiés n'ont pas été retrouvés après analyse de 200 chromosomes témoins. La moitié de ces mutants sont localisés sur le gène SCN5A. La caractérisation fonctionnelle de chacun d'entre eux est actuellement en cours. Cette étude confirme que les mutations affectant les gènes du QT long représentent une des causes génétiques majeures des cas de Mort Subite du Nourrisson. Si l'autopsie ne permet pas de déterminer une cause au décès d'un nourrisson, l'étude médico-légale devrait donc être complétée par l'analyse des gènes impliqués dans le syndrome du QT Long. L'identification de ces mutations pourrait donc permettre de réduire le nombre de morts subites inexpliquées du nourrisson et permettre d'envisager de nouvelles perspectives physiopathologiques.

Mots-clés : mort subite du nourrisson, QT long, arrythmies.

GP310 / 25. UN NOUVEAU LOCUS POUR LES ÉPILEPSIES GÉNÉRALISÉES AVEC CONVULSIONS FÉBRILES (GEFS+) EN 8P23-P21

S. Baulac (1), I. Gourfinkel-An (2), P. Couarch (1), M. Baulac (2), O. Dulac (3), R. Nabbout (3), E. LeGuern (1)

(1) INSERM, UMR 679, Hôpital de la Pitié-Salpêtrière, Paris, France ; (2) Center of Epileptology, AP-HP, Hôpital de la Pitié-Salpêtrière, Paris, France ; (3) Neuropediatrics department, AP-HP, Hôpital Necker-Enfants Malades, Paris, France 
Le contexte familial GEFS+ (Generalized epilepsy with febrile seizures plus) associe des convulsions fébriles à des crises épileptiques afébriles À ce jour, des mutations dans 3 gènes, SCN1A, SCNIB et GABRG2, ont été identifiées suggérant une hétérogénéité génétique. Nous avons réalisé une cartographie génétique à l'aide de marqueurs microsatellites espacés de $10 \mathrm{~cm}$ dans sept familles françaises dont le phénotype était compatible avec le tableau GEFS+ et une transmission autosomique dominante. Un nouveau locus a été identifié sur le bras court du chromosome 8, en 8p23-p21 après exclusion du reste du génome dans la famille la plus informative. Le génotypage des six autres familles avec les marqueurs de la région suggère qu'une seconde famille est liée à ce locus, permettant ainsi de réduire l'intervalle candidat à $9 \mathrm{Mb}$. Le séquençage des gènes candidats localisés dans la région est en cours.

Mots-clés : épilepsie, convulsions fébriles, locus.

P311 / 26. LONG QT SYNDROME CAUSED BY LARGE DELETIONS INCLUDING THE KCNH2 (HERG) GENE UNDETEC TABLE BY CURRENT POLYMERASE CHAIN REACTIONBASED EXON-SCANNING METHODOLOGIES

S. Schmitt (1, 2), F. Briec (1), M. Le Cunff (1), C. Vieyres (3), C. Le Caignec $(1,2)$, F. Kyndt $(1,2,4)$, H. Le Marec $(1,4,5)$, V. Probst $(1,4$, 5), J.J. Schott $(1,2,4)$

(1) Inserm U533, l'institut du thorax, Faculté de médecine Nantes, France ; (2) Laboratoire de Génétique Moléculaire Service de Génétique Médicale ; (3) Cabinet de cardiologie Angoulême, France ; (4) Centre de référence de maladies rares; (5) Clinique cardiologique, l'institut du thorax, $\mathrm{CHU}$ de Nantes, France

Long-QT syndrome (LQTS) is an inherited cardiac arrhythmia characterized by a prolonged QT interval on the surface ECG associated with syncope and sudden death from torsades de pointes polymorphic ventricular tachycardia. It is estimated to affect 1 in 5000 individuals. LQTS may be caused by mutations in 4 major genes encoding $\mathrm{K}+$ channels pore forming (KCNQ1, KCNH2) and auxiliary (KCNE1, KCNE2) subunits and in the gene encoding the cardiac specific Na+ channel SCN5A. Genetic testing for congenital LQTS has been performed in research laboratories for the past decade. Approximately $75 \%$ of patients diagnosed with LQTS have mutations in one of five LQTS genes. Possible explanations for the remaining genotype-negative cases include LQTS mimickers, novel LQTScausing genes, unexplored regions of the known genes, and genetic testing detection failures. The purpose of this study was to determine the relative copy number in the 5 major LQTS genes in a series of 100 mutationnegative LQTS probands. A multiplex ligation-dependent probe amplification (MLPA) approach was used using SALSA P114 MLPA kit (MRC Holland, Amsterdam, The Netherlands). Aberrant exon copy numbers were confirmed by Quantitative Multiplex PCR of Short Fluorescent Fragment (QMPSF). Array based CGH analysis was performed using Agilent Human Genome $244 \mathrm{~K}$ Micro arrays to further map the genomic rearrangements. Whenever possible, familial investigation was performed to test segregation of the copy number variation. This study identified two large deletions in $K C N H 2$ gene in 2 probands. A $K C N H 2$ deletion spanning exon 4 to 14 was identified in a 20 years old symptomatic woman with QTc $>554$ milliseconds. QMPSF analysis of exon 15 identified a deletion of the last $\mathrm{KCNH} 2$ exon. CGH analysis identified a much larger genomic deletion of $650 \mathrm{~Kb}$ deletion including $K C N H 2$ exon 4 to 15 but also 19 additional genes downstream of $\mathrm{KCNH} 2$ including the amiloride binding protein 1 gene $(A B P 1)$ and a cluster of genes encoding immunity-associated proteins (GIMAPs). Familial and clinical investigations identified 3 additional affected individuals with a $K C N H 2$ deletion. A second large deletion was identified in a second proband with a QTc $=478$ milliseconds who experienced syncope after emotional stress. MLPA analysis identified the deletion from exon 1 to 14 and exon 15 QMPSF analysis confirmed full length deletion of $\mathrm{KCNH} 2$ gene. Using Agilent array CGH $244 \mathrm{~K}$, the size of the deletion was estimated to be $145 \mathrm{~Kb}$. 5 ' breakpoint maps between $K C N H 2$ and NOS3 genes intergenic region whereas 3' breakpoint maps after the $A B P I$ gene. No familial investigation was possible since the proband was adopted. Both genomic deletions were not found in 100 controls and no deletions or duplications were identified in the KCNQ1,KCNE1, KCNE2 or $S C N 5 A$ genes. Both deletions are expected to be non functional thereby decreasing IKr current in ventricular cardiomyocytes suggesting haploinsufficiency as the most likely mechanism leading to LQTS in both probands and relatives. Further clinical investigations are currently underway to investigate for additional phenotypes in all patients. Despite a quit limited number of LQTS probands screened in this study, the identification of 2 large deletions in LQTS genes strongly suggests screening for copy number variant in mutation negative LQTS probands.

Mots-clés : cœur, arythmie, biologie moléculaire.
EP312 / 35. SEARCH FOR THE RP25 GENE IN A CONSANGUINEOUS FAMILY WITH AUTOSOMAL RECESSIVE RETINITIS PIGMENTOSA

E. Lafont (1), A. Sénéchal (1), C. Barbezier (1), G. Humbert (1), D. Hillaire (1), C.P. Hamel (1)

(1) INSERM U583 INM, Hôpital Saint-Éloi, Montpellier

Retinitis Pigmentosa (RP) belongs to the group of pigmentary retinopathies and is the most common inherited retinal dystrophy. This disease is characterized by a progressive loss of vision, initially manifesting as night blindness and a reduction of the peripheral visual field. There are only $50-60 \%$ of all cases of autosomal dominant RP and 30-60\% of all cases of autosomal recessive (ar) RP for which the gene is identified. To date 22 genes and loci have been implicated in arRP. We performed locus exclusion followed by a genome wide scan in a consanguineous Tunisian family with arRP comprising 31 members in six generations, among which 18 were affected. We found evidence of linkage on $6 \mathrm{q}$ (lod score at theta $=$ 0 of 6.60 for D6S257 and of 6.35 for D6S1053) corresponding to the previously described RP25 locus, and for which the gene remains to be identified. We then searched for the mutation in a $6.7-\mathrm{Mb}$ interval. We have studied the 10 candidate genes located in this interval including EGFL11, since this gene is strongly expressed in the retina. No causative mutation was found. We are currently sequencing $17 \mathrm{LOC}$ sequences. The discovery of new genes will provide perspectives to understand the pathophysiology of RP and to develop therapeutic approaches.

Mots-clés : candidate genes, RP25, genetic disease.

IP313 / 36. RECHERCHE DE MUTATIONS MITOCHONDRIALES CHEZ DES PATIENTS TUNISIENS ATTEINTS DU SYNDROME DE LEIGH

E. Mkaouar-Rebai (1), F. Kammoun (2), C. Triki (2), F. Fakhfakh (1)

(1) Laboratoire de Génétique Moléculaire Humaine, Faculté de Médecine de Sfax, Tunisia; (2) Service de Neurologie, CHU Habib Bourguiba de Sfax, Tunisia

Le syndrome de Leigh est une maladie neurologique progressive caractérisée par des lésions neuropathologiques qui débute typiquement chez le nourrisson de moins d'un an mais il existe de rares cas de début tardif, jusqu'à l'âge adulte. Parmi les premiers signes de la maladie, on note souvent une régression psychomotrice, une hypotonie générale ou axiale, une régression des acquisitions motrices, et des vomissements. L'IRM montre des lésions du type hypersignaux en $\mathrm{T} 2$ des noyaux gris, en particulier des putamens et de la protubérance. Une rétinopathie, une atrophie optique et une surdité sont souvent présentes. Les causes du syndrome de Leigh sont multiples, mais dans tous les cas, ils impliquent un défaut de la production énergétique aérobie. Les gènes altérés responsables peuvent être d'origine mitochondriale ou nucléaire ; ils codent pour l'une des sousunités du complexe de la pyruvate déshydrogénase ou des complexes respiratoires I ou II, ou encore pour une protéine intervenant dans l'assemblage du complexe $\mathrm{V}$. Les mutations mitochondriales associées au syndrome de Leigh sont multiples et elles touchent essentiellement les gènes ND3, ND4, ND4L, ND5, ND6, l'ATPase 6, l'ARNtLeu(UUR), l'ARNtTrp et l'ARNtLys mitochondriaux. L'étude a porté sur 15 patients Tunisiens présentant un tableau clinique évocateur d'un syndrome de Leigh. Le diagnostic a été basé sur des données neurologiques, radiologiques et biochimiques. La recherche de mutations mitochondriales associées à cette maladie a été effectuée par PCR-RFLP ou par séquençage automatique. Les résultats ont montré l'absence de toute mutation mitochondriale décrite dans le syndrome de Leigh dans les gènes testés. Cependant, nous avons détecté 58 polymorphismes connus au niveau des gènes de l'ATPase $6, N D 3, N D 4 L, N D 4, N D 5, N D 6$. Tous ces 58 polymorphismes sont rapportés dans les bases de données de l'ADN mitochondrial. Nous avons aussi repéré 16 nouveaux polymorphismes et 2 nouvelles mutations responsables d'un changement d'acide aminé au niveau des gènes de NADH déshydrogénase 2 et de l'ATPase 8 . Nous avons soumis toutes ces nouvelles variantes dans la base de données de l'ADN mitochondrial: www.mitomap.org. Elles peuvent être consultées en accédant à http://www.mitomap.org/cgi-bin/mitomap/tbl15gen.pl sous les références suivantes: \#2007072403, \#2007072404, \#2007072406, \#2007072407, \#2007072408, \#20070724010, \#20070724011, \#20070724012, \#20070724013, \#20070327005, \#20070327006, \#20070327008, \#20070327009, \#20070327010, \#20070327011, \#20070327012, \#20070609002, \#20070609003

La recherche par modélisation de l'effet des ces deux mutations est en cours.

Mots-clés: mutations mitochondriales, syndrome de Leigh, maladie mitochondriale. 
IP314 / 40. FRÉQUENCE ÉLEVÉE DE MICRORÉARRANGEMENTS DE LA RÉGION AZFC DANS DEUX POPULATIONS INFERTILE ET NORMOZOOSPERMIQUE FRANÇAISES

V. Roze (1), J.L. Bresson (1, 2), F. Fellmann (3)

(1) EA MENRT 3185 Génétique et Reproduction, Faculté de Médecine et de Pharmacie: (2) Service de Génétique, Histologie, BDR, CHU, place St-Jacques, 25030 Besançon, France; (3) Service de génétique médicale, CHUV, 1011 Lausanne, Suisse

La région AZFc du bras long du chromosome $\mathrm{Y}$ est une des régions les moins stables du génome humain. Elle est composée de multiples palindromes entraînant différents types de réarrangements: délétions complètes ou partielles, duplications complètes ou partielles ou encore des évenements de délétion/duplication. L'impact de ces réarrangements sur l'infertilité masculine est encore discuté. Les gènes les plus étudiés dans $\mathrm{AZFc}$ sont $D A Z$ et $C D Y 1$; présents en 4 et 2 exemplaires respectivement. Nous avons recherché les microréarrangements d'AZFc dans 2 populations : 344 patients infertiles et 136 contrôles. Pour cela, l'analyse par PCR de marqueurs spécifiques d'AZFc, l'analyse de polymorphismes SNVs de $D A Z$ et $C D Y I$ ainsi que le dosage du nombre de copies de ces gènes par PCR quantitative «en temps réel » (Q-PCR) ont été réalisés. Tous les patients ont été haplotypés pour les polymorphismes YAP, $12 \mathrm{f} 2$ et $92 \mathrm{R} 7$ et les individus présentant un réarrangement d'AZFc également pour les polymorphismes Tat, M9 et SRY-4064, définissant 5 haplogroupes de l'Y: E, J, $\mathrm{K}(\mathrm{xN} 3, \mathrm{P}), \mathrm{N} 3$ et $\mathrm{P}$ et un paragroupe $\mathrm{Y} *(x \mathrm{D}, \mathrm{E}, \mathrm{J}, \mathrm{K})$. Quarante-deux réarrangements $(8,7 \%)$ ont été identifiés représentant une fréquence élevée de recombinaisons, avec des fréquences de délétions et de duplications similaires dans les deux populations. L'analyse des polymorphismes SNVs de DAZ et $C D Y 1$ ainsi que le dosage du nombre de copies de $C D Y 1$ par Q-PCR nous ont permis de mieux caractériser ces réarrangements et leurs mécanismes.Les réarrangements complexes (délétion /duplication) ont été identifiés uniquement chez les patients infertiles, incluant deux de l'haplogroupe N3 et deux de l'haplogroupe J. Cette étude apporte des données complémentaires sur les réarrangements de la région $\mathrm{AZFc}$ et leurs potentiels impacts sur l'infertilité masculine.

Mots-clés : rearrangements chromosomiques, infertilité masculine, chromosome Y.

口P315 / 45. RECHERCHE DE MUTATIONS DANS LE GÈNE MECP2 CHEZ DES PATIENTS TUNISIENS ATTEINTS DU SYNDROME DE RETT

N. Fendri-Kriaa (1), Z. Abdelkafi (2), I. Ben Rebeh (2), F. Kammoun (1), C. Triki (1), F. Fakhfakh (2)

(1) Unité de Neuropédiatrie, CHU Habib Bourghiba de Sfax; (2) Laboratoire de génétique moléculaire humaine, Faculté de Médecine de Sfax

Le syndrome de Rett (SR) est une maladie neurodégénérative chronique d'origine inconnue qui touche presque exclusivement les filles. Il est caractérisé par un retard psychomoteur apparaissant entre l'âge de 6 et 18 mois avec plusieurs critères de diagnostic dont la stéréotypie des mains est le signe le plus caractéristique. Il constitue l'une des causes les plus connues du retard mental chez les femmes avec une fréquence de $1 / 10000$ à $1 / 15000$. Les cas sont le plus souvent sporadiques. Ce syndrome de Rett est une maladie héréditaire liée au chromosome $\mathrm{X}$ qui se transmet selon un mode dominant. Elle est causée essentiellement par des mutations dans le gène $\mathrm{MeCP} 2$ (en Xq28) codant pour une protéine appelée Methyl-CpGBinding Protein 2 (MeCP2). L'objectif de ce travail est de chercher les mutations et/ou les polymorphismes au niveau du gène $\mathrm{MeCP} 2$ chez deux patientes Tunisiennes (P1 et P2) atteintes du syndrome de Rett. Cette recherche a été réalisée par séquençage direct des 4 exons du gène et des séquences introniques flanquantes. Les résultats de séquençage ont montré l'absence de mutations et polymorphismes dans les régions explorées du gène chez la patiente $\mathrm{P} 2$. Cependant, la patiente $\mathrm{P} 1$ a montré la présence de la mutation faux sens R306C qui change l'acide aminé Arginine par une Cystéine. Cette mutation est une transition $(916 \mathrm{C}>\mathrm{T})$ qui a été décrite comme la première mutation qui touche le domaine TRD (Transcription Repression Domain) de la protéine MeCP2. D'autre part, les résultats ont montré l'absence de cette mutation chez les parents de la patiente P1. Il s'agit donc d'une mutation de novo. En conclusion, cette première étude sur le syndrome de Rett en Tunisie a permis de confirmer l'implication du gène $\mathrm{MeCP} 2$ dans le syndrome de Rett. Une analyse des régions promotrices et non traduites du gène $\mathrm{MeCP} 2$ est prévue pour chercher la mutation chez la patiente $\mathrm{P} 2$.
EP316 / 47. ALTÉRATIONS DU MÉTABOLISME DE L'ARNM DU GÈNE LAMA2 GÉNÉRANT UN DÉFICIT TOTAL EN LAMININE ALPHA 2 ET UNE ATTEINTE SÉVẼRE DE DYSTROPHIE MUSCULAIRE CONGÉNITALE

O. Siala (1, 2), N. Louhichi (1), C. Triki (3), M. Morinière (2), F. Fakhfakh (1)*, F. Baklouti (2)* ; * Les deux derniers auteurs doivent être considérés comme équivalents

(1) Laboratoire de Génétique Moléculaire Humaine, Faculté de Médecine de Sfax; (2) Université Lyon 1, Lyon, F-69003, France ; CNRS, UMR5534, Centre de génétique moléculaire et cellulaire; (3) Service de Neurologie, CHU Habib Bourguiba de Sfax

La Dystrophie Musculaire Congénitale (DMC) est une maladie neuromusculaire rare et hétérogène à transmission autosomique récessive. À une hétérogénéité clinique est associée une hétérogénéité génétique, donnant les différentes formes décrites. Cependant, toutes les formes montrent une faiblesse musculaire et une hypotonie générale dès la naissance ou dans les 6 mois qui la suivent, avec un taux de CPK élevé. Cinquante pourcent des cas atteints montrent un déficit total primaire en laminine alpha 2 , causé par des mutations sur le gène LAMA2 (6q22), et donnant naissance à la forme MDC1A. Nous présentons dans ce travail une atteinte sévère et chevauchante de DMC chez une patiente ayant présenté une scoliose lombaire et des difficultés respiratoires, menant à son décès à l'age de 10 ans. Une immunofluorescence réalisée sur des coupes de biopsie musculaire de la patiente ainsi que d'un témoin normal a été réalisée avec deux anticorps monoclonaux spécifiques de la laminine alpha 2 . Une étude de liaison génétique a été réalisée utilisant des marqueurs microsatellites spécifiques des différentes formes décrites de DMC y compris la forme $\mathrm{MDC} 1 \mathrm{~A}$. Le séquençage des 65 exons codants du gène LAMA2 ainsi que leurs jonctions introniques a été également effectué. Afin d'explorer l'impact des mutations retrouvées dans le gène LAMA2 sur le métabolisme de l'ARNm, une approche ex-vivo de construction de minigène suivie d'une transfection cellulaire transitoire de cellules Hela et de RT-PCR ont été requises. Les études de liaison génétique n'ont montré de liaison évidente à aucune des formes décrites de DMC. Cependant, la déficience totale en laminine alpha 2 retrouvée chez la patiente était en faveur de son atteinte par la forme MDC1A. Le séquençage des 65 exons du gène LAMA2 ainsi que leurs jonctions introniques a révélé que la patiente est hétérozygote composite pour deux nouvelles mutations: la première, héritée du coté maternel, est une délétion frameshift sur l'exon 57 (8007delT) générant un codon stop. La deuxième est une mutation de novo consistant en une délétion de $7 \mathrm{pb}$ dans l'intron 17 (c.2450+5_c.2450+11del). Les résultats de l'étude fonctionnelle par RT-PCR a montré que la mutation (c. $2450+5$ c. $2450+11$ del) entraîne un skipping total de l'exon17. Les résultats de l'immunofluorescence ainsi que ceux de l'approche fonctionnelle suggèrent que les deux mutations sont en trans, génèrant des $\mathrm{ARNm}$ nonsens qui sont potentiellement cibles à la dégradation par NMD (nonsens mRNA meditaed decay). L'ensemble de ces résultats montrent que la mutation (c. $2450+5$ c. $2450+11 \mathrm{del}$ ) altère le métabolisme de l'ARNm du gène LAMA2 malgré sa présence hors des dinucléotides consensus des sites 5 et 3' d'épissage (5'ss et 3'ss). En effet, cette délétion de 7pb altére l'extrémité 3 ' du consensus d'épissage en 5' qui semble être cruciale dans le recrutement de la protéine U6snRNP. L'emplacement des U1snRNA et U6snRNA au 5'ss est nécessaire pour activer le spliceosome, en apportant le site catalytique dans la région proche du 5'ss.

Mots-clés : dystrophie musculaire congénitale, exon skipping, non-sens mRNA mediated decay.

PP17 / 48. CORRÉLATION PHÉNOTYPE-GÉNOTYPE DANS LE DÉFICIT HÉRÉDITAIRE EN PYRUVATE CARBOXYLASE

S. Monnot $(1,2)$, V. Serre (1), B. Chadefaux (3), S. Romano (4), P. de Lonlay (4), J. Aupetit (3), V. Paquis (2), A. Munnich (1), J.-P. Bonnefont (1)

(1) Service de génétique médicale, hôpital Necker-Enfants Malades, Paris : (2) Service de génétique médicale, hôpital l'Archet 2, Nice; (3) Service de biochimie métabolique, hôpital Necker-Enfants Malades, Paris; (4) Service de maladies métaboliques pédiatriques, hôpital Necker-Enfants Malades, Paris

Le déficit primaire en pyruvate carboxylase (PC) (non biotine-sensible), enzyme catalysant la première étape de la néoglucogenèse, est une anomalie génétique rare de transmission autosomique récessive. Trois phénotypes ont été décrits selon la sévérité de l'expression clinique. Seulement 6 mutations du gène codant pour la PC ont été rapportées à ce jour. Parmi les quatre patients de la présente étude, trois enfants d'origine caucasienne (patients $1,3,4)$ présentaient la forme la plus sévère, dite forme "française », de la maladie. Deux sont décédés dans la première semaine de vie et le troisième à l'âge de 7 mois, dans un contexte d'acidose lactique 
majeure. La dernière patiente, issue d'un couple consanguin originaire d'Arabie Saoudite (patiente 2) présentait à l'age de 2 ans un retard de développement psychomoteur et des épisodes récurrents d'acidose lactique (forme dite « américaine »). L'activité de la PC était indétectable dans les fibroblastes de tous les patients. Le séquençage direct des 23 exons du gène codant pour la $\mathrm{PC}$ a permis d'identifier 6 nouveaux variants, i) l'un à l'état homozygote chez la patiente 2 (forme américaine), à savoir c.467G >A (exon 3, p.Arg156Gln), et ii) les 5 autres en situation d'hétérozygotie composite, à savoir c. $911 \mathrm{~A}>\mathrm{G}$ (exon 7, p.Tyr304Cys) et IVS7-1G $>\mathrm{T}$ (intron 7, anomalie d'épissage) chez le patient $1, \mathrm{c} .1748 \mathrm{G}>\mathrm{T}$ (exon 12, p.Arg583Leu) et c.2876dupT (exon 17, stop prématuré) chez le patient 3, et c.3392_3400del9nt (exon 20, stop prématuré) pour le patient 4 (deuxième mutation non identifiée). Les arguments suggérant la responsabilité des variants de séquence identifiées vis-à-vis du phénotype clinique sont i) pour la délétion, la prédiction d'un décalage du cadre avec survenue d'un codon stop prématuré, ii) pour l'anomalie d'épissage et la duplication, la mise en évidence d'un phénomène de « RNA decay » après séquençage du cDNA, iii) pour les mutations «faux-sens », 1/ leur absence sur 100 chromosomes témoins, 2/ leur localisation dans des domaines protéiques très conservées, 3 / la prédiction des effets de ces mutations sur la conformation de la protéine après modélisation in silico, fondée sur les similarités de la PC humaine (non cristallisée) avec la sous-unité biotine carboxylase de la PC d'Aquifex aeolicus et avec la transcarboxylase de Propionibacterium shermanii, dont la structure tridimensionnelle est connue. Ces données, jointes à celles (rares) de la littérature, suggèrent que les mutations tronquantes sont plus fréquemment associées à la forme « française » de la maladie, alors que les mutations faux-sens prédomineraient dans la forme « américaine ».

Mots-clés : pyruvate carboxylase, corrélation génotype-phénotype, analyse mutationnelle.

IP318 / 55. LES MUTATIONS DANS LE GÈE TCF 8 ENTRAîNENT UNE EXPRESSION ECTOPIQUE DE LA E-CADHÉRINE AU COURS DE LA DYSTROPHIE POSTÉRIEURE POLY. MORPHE DE LA CORNÉE

S. Valleix (1), B. Nedelec (1), C.M. Bera (1), F. Niel (1), B. Kantelip (2), B. Delbosc (3), M. Muraine (4), G. Renard (5), M. Delpech (1)

(1) Service de Biochimie et Génétique Moléculaire et U567-CHU Cochin de Paris ; (2) Service d'Anatomie Pathologique-CHU Besançon; (3) Service d'Ophtalmologie-CHU Besançon ; (4) Service d'Ophtalmologie-CHU Rouen ; (5) Service d'Ophtalmologie-CHU Hôtel-Dieu de Paris

La dystrophie postérieure polymorphe de la cornée (PPCD) est une dystrophie héréditaire autosomique dominante qui affecte l'endothélium et la membrane de Descemet de la cornée. Au cours de cette maladie, les cellules endothéliales de la cornée, qui normalement forment une monocouche de cellules hexagonales amitotiques, subissent une transformation épithéliale avec changement morphologique, prolifération et élaboration d'une membrane basale anormale. Récemment, le gène TCF 8 (GeneBank accession number NM_030751) s'est révélé être un des gènes impliqués dans cette pathologie. TCF8 est un répresseur majeur de la transcription de nombreux gènes dont certains codent pour des collagènes et des molécules impliquées dans les jonctions intercellulaires, notamment la E-cadhérine. Les souris invalidées pour ce gène présentent de nombreuses anomalies du squelette, des anomalies des cellules $\mathrm{T}$ et meurent peu après la naissance montrant que TCF8 joue un rôle majeur au cours du développement embryonnaire. Parallèlement, $T C F 8$ s'est révélé être crucial dans les mécanismes moléculaires impliqués dans la transition épithélio-mésenchymateuse (EMT), mécanisme mis en jeu dans de nombreux processus de développement pour la mise en place des tissus, mais également anormalement réactivé dans la progression tumorale. Nous présentons l'étude de 5 familles atteintes de PPCD chez lesquelles nous avons identifié des mutations nouvelles du gène $T C F 8$. Dans 2 familles, nous rapportons également des manifestations extraoculaires qui récapitulent en partie le phénotype des souris invalidées pour $t c f 8$. De plus, à partir de spécimens de cornée pathologiques obtenus lors de greffes de cornée de ces patients atteints de PPCD nous avons mené une étude histologique en microscopie optique, en microscopie électronique ainsi qu'une étude immunohistochimique. Nous montrons que chez ces patients mutés dans TCF8, les cellules endothéliales ont acquis un phénotype épithélial exprimant anormalement des cytokératines et qu'elles ont également anormalement proliféré. De plus, ces cellules endothéliales pathologiques ont élaboré une membrane de Descemet anormale suggérant que la pathologie a débuté avant la $12^{\mathrm{e}}$ semaine de gestation. En microscopie électronique ce phénotype épithélial des cellules endothéliales pathologiques s'accompagne de l'apparition anormale de jonctions cellulaires de type desmosomes et jonctions adhérentes. Sur le plan moléculaire, nous avons mis en évidence pour la $1^{\mathrm{re}}$ fois que ces cellules endothéliales pathologiques ayant un gène $T C F 8$ muté expriment anormalement la E-cadhérine. Au cours de cette étude, nous avons également découvert que les patients mutés dans TCF8 présentent également une pathologie de l'épithélium cornéen.

Mots-clés : dystrophie cornéenne héréditaire, gène $T C F 8$, E-cadhérine.

EP319 / 61. MUTATION MONOALLÉLIQUE DU GÈNE BUB1B DANS UN CAS FAMILIAL DE MVA/PCS

E. Bohers (1), A. Moncla (2), R. Sesboüé (1), N. Philip (2), T. Frebourg (1)

(1) Inserm U614, Faculté de Médecine et de Pharmacie, Institut Hospitalo-Universitaire de Recherche Biomédicale de Rouen; (2) Département de Génétique Médical Hôpital des Enfants de la Timone, Marseille

Le point de contrôle du fuseau mitotique est un mécanisme de surveillance essentiel permettant de maintenir un nombre correct de chromosomes au cours du cycle cellulaire. L'une des protéines majeures de ce point de contrôle est la kinase BUBR1 (codée par le gène $B U B I b$ ). Récemment, des mutations bialléliques du gène $B U B I b$ ainsi que des mutations hétérozygotes associées à un variant spécifique en trans ont été identifiées dans le cadre du syndrome MVA (mosaic variegated aneuploidy). Le syndrome MVA, dont la transmission est considérée comme autosomique récessive, est caractérisée par des aneuploïdies constitutionnelles en mosaïque associées à un retard sévère de croissance intra-utérine, une microcéphalie, une prédisposition aux cancers et, dans la plupart des cas, à une séparation prématurée des chromatides (PCS). Nous avons réalisé une analyse extensive du locus BUBlb dans le cadre d'une récurrence de syndrome MVA avec PCS au sein de la même fratrie. Le premier fotus présentait une microcéphalie avec une malformation de Dandy-Walker et le caryotype sur liquide amniotique a mis en évidence des aneuploïdies multiples en mosaïque dans $72 \%$ des cellules. La surveillance échographique de la seconde grossesse a révélé une microcéphalie, une anomalie de la fosse postérieure et retard de croissance et le caryotype a objectivé des aneuploïdies multiples dans $71 \%$ des cellules, confirmant la récurrence de MVA. Des images typiques de PCS ont pu être détectées dans $21 \%$ des cellules fotales et l'évaluation retrospective du liquide amniotique de la première grossesse a confirmé la PCS dans $33 \%$ des cellules. Le criblage de la séquence codante du gène $B U B I b$, réalisé chez les 2 foetus n'a permis de mettre en évidence chez les 2 fœtus qu'une seule mutation hétérozygote délétère touchant le site canonique donneur d'épissage de l'exon 7 (c. $968+1 \mathrm{G}>\mathrm{A})$. Une QMPSF explorant les 23 exons de $B U B I b$ a exclu l'existence d'un remaniement génomique hétérozygote. Ne disposant pas de l'ADN des parents, nous avons mis au point une PCR quantitative des deux marqueurs $26020 \mathrm{GT} / \mathrm{rs} 35585912$ et D15S994 localisés respectivement à $54 \mathrm{~kb}$ en amont et $69 \mathrm{~kb}$ en aval du gène $B U B 1 \mathrm{~b}$. Cette analyse a révélé que les deux fotus ne partageaient en fait qu'un seul allèle de $B U B I b$. Ainsi, ces résultats suggèrent que le déterminisme génétique du syndrome MVA est probablement complexe : autosomique récessif dans certaines familles, résultant de l'association d'une mutation nulle et d'un variant hypomorphe dans d'autres, il pourrait être même oligogénique. Cette hétérogénéité pourrait s'expliquer par la biologie de la protéine BUBR1 qui, comme toutes les protéines impliquées dans le fuseau mitotique, est particulièrement sensible au dosage.

Mots-clés : MVA, BUB1b, hétérogénéité génétique.

\section{IP320 / 62. ÉTUDE FONCTIONNELLE DES CELLULES ENDO- THÉLIALES PROVENANT DU FOIE DE PATIENTS ATTEINTS DE MALADIE DE RENDU-OSLER}

G. Lesca $(1,2)$, B. Bancel $(1,3)$, O. Boillot (4), S. Giraud (2), M.-F Bourgeade (5), H. Plauchu (6), C. Trépo (1), M.-J. Marion (1)

(1) Inserm U871, Lyon; (2) Laboratoire de génétique, Hôpital ÉdouardHerriot, Hospices Civils de Lyon; (3) Laboratoire d'anatomie pathologique, Hôpital de la Croix-Rousse, Hospices Civils de Lyon ; (4) Fédération des spécialités digestives, Hôpital Edouard Herriot, Hospices Civils de Lyon; (5) Inserm U542, Hôpital Paul-Brousse, Villejuif; (6) Service de génétique, Hôtel-Dieu, Hospices Civils de Lyon

La maladie de Rendu-Osler ou HHT (hereditary hemorrhagic telangiectasia) est une maladie vasculaire autosomique dominante dont la prévalence dépasse 1/8 000. Elle est caractérisée par des malformations artérioveineuses et s'exprime le plus souvent par des épistaxis spontanés et répétés et des télangiectasies cutanéo-muqueuses. Le pronostic de la maladie est surtout déterminé par la survenue de complications pulmonaires, hépatiques, intestinales ou cérébrospinales. La HHT est principalement causée par les mutations germinales des gènes $E N G$ et $A C V R L I$, codant respectivement pour l'endogline et ALK1, qui sont des récepteurs appartenant à la superfamille du TGF-beta. Il est généralement admis que les mutations produisent une haploinsuffisance. Les 
atteintes hépatiques sont plus fréquentes chez les patients porteurs d'une mutation du gène ACVRL1. Elles ont longtemps été sous-estimées et, si elles demeurent silencieuses durant plusieurs décennies, elles peuvent être à l'origine d'une insuffisance cardiaque ou d'une cholangite ischémique nécessitant le recours à une transplantation orthotopique. Bien que ces lésions hépatiques soient dépistées de plus en plus précocement, leur histoire naturelle est encore très mal connue. Les cellules endothéliales pourraient jouer un rôle clé dans leur initiation et leur progression. Le but de notre travail était d'étudier les conséquences des mutations germinales au niveau des cellules provenant du foie explanté de quatre patients ayant bénéficié d'une transplantation orthotopique et porteurs de mutations différentes du gène ACVRL1. Nous avons pu isoler des cellules endothéliales et des myofibroblastes que nous avons caractérisées d'après leur morphologie et l'expression de marqueurs spécifiques en immunofluorescence et en RT-PCR. Nous avons recherché l'existence d'une mutation sur le deuxième allèle ou une perte d'hétérozygotie par QMPSF. Nous avons effectué plusieurs tests fonctionnels classiques de l'angiogénèse : test de prolifération et de synthèse d'ADN, test de blessure, test de tubulogénèse sur Matrigel. Nous avons étudié l'expression d'endogline, d'ALK1 et des Smad1 et 2 en western blot et en immunofluorescence. Nous avons également étudié la sécrétion de TGF-beta actif par les cellules endothélailes et les myfibroblastes. Nos résultats montrent que les cellules endothéliales des patients ont une morphologie normale et ne présentent pas d'anomalie majeure de prolifération, de migration ou de capacité à former des structures tubulaires sur Matrigel. On ne retrouve pas de mutation sur le deuxième allèle. Le taux normal d'expressoin d' $A L K I$ en western blot n'est pas en faveur d'une haploinsuffisance. Il existe une sécrétion de TGF-beta actif, tant par les cellules endotheliales que par les myofibroblastes des patients, qui n'est pas observée chez les témoins. En conclusion, les cellules endotheliales provenant du foie de patients HHT ne présentent pas d'altération morphologique et fonctionnelle majeure, in vitro, et en particulier en ce qui concerne les voies de signalisation du TGF-beta. Nos résultats suggèrent que les lésions vasculaires de la maladie résulteraient de perturbations des relations entre les cellules endothéliales et leur environnement tissulaire, débutant dès la période embryonnaire.

Mots-clés : rendu-Osler, cellule endotheliale, ACVRLI.

EP321 / 68. IMPLICATION D'UNE DÉLÉTION DU PROMOTEUR DE SLC39A4 DANS L'ACRODERMATITE ENTÉROPATHIQUE

S. Schmitt (1), M. Giraud (1), C. Scoul (2), J. Morissard (3), S. Küry (2), S. Bezieau $(1,2)$

(1) Service de Génétique Médicale-CHU Nantes ; (2) Laboratoire d'étude du polymorphisme de l'ADN (LEPA)-Faculté de médecine de Nantes; (3) INSERM U533-Nantes

L'acrodermatite entéropathique (AE) est une maladie génétique autosomique récessive rare due à des mutations dans le gène SLC39A4 (8q24.3) codant pour un transporteur du zinc exprimé majoritairement au niveau duodénal, jejunal et rénal. Il s'agit d'une affection liée à un déficit du zinc caractérisé sur le plan clinique par une dermite acrale et péri-orificielle, une diarrhée et une alopécie généralisée. Le séquençage direct du gène permet l'identification d'une anomalie moléculaire dans environ $80 \%$ des cas. Afin d'augmenter le taux de détection des mutations, la recherche de grands réarrangements a été développée par QMPSF au sein du laboratoire et a permis l'identification d'une délétion de $1.9 \mathrm{~kb}$ située $3 \mathrm{~kb}$ en amont du promoteur. L'analyse in-silico a permis la mise en évidence de 7 éléments MRE (Metal Response Elements) dans cette région. Ces motifs sont impliqués dans la régulation de l'expression des gènes ce qui suggère un rôle potentiel dans le mécanisme moléculaire de l'AE. Parmi notre cohorte de 42 cas index, cette délétion est retrouvée à l'état homozygote avec une fréquence allèlique de $7.1 \%$ contre $0.36 \%$ dans une population de 275 témoins et avec une fréquence de $5.95 \%$ à l'état hétérozygote contre $4.9 \%$ pour les témoins. Pour les patients $\mathrm{AE}$ présentant la délétion à l'état homozygote, un variant c. $192+19 \mathrm{G}>\mathrm{A}$ est retrouvé systématiquement en déséquilibre de liaison à la différence de la population témoin délétée. En conclusion, la délétion du promoteur observée chez les patients atteints d'AE correspondrait à un polymorphisme associé à la mutation c. 192+19G >A . Des études complémentaires de ségrégation dans les familles AE et le dosage du zinc plasmatique chez les hétérozygotes permettrait de statuer sur un éventuel rôle modulateur de cette délétion dans l'absorption du zinc.

Mots-clés : acrodermatite enteropathique, SLC39A4, délétion.

IP322 / 70. EFFETS FONDATEURS RÉGIONAUX POUR LA MALADIE DE RENDU-OSLER LIÉE AUX MUTATIONS DU GÈNE ACVRLI CHEZ LES PATIENTS FRANÇAIS ET ITALIENS

G. Lesca (1), E. Genin (2), C. Blachier (1), C. Olivieri (3), F. Coulet (4), G. Brunet (5), S. Dupuis-Girod (6), E. Buscarini (7), F. Soubrier (4), A.
(1) Laboratoire de Génétique, Hôpital Édouard-Herriot, Hospices Civils de Lyon; (2) INSERM U535, Villejuif; (3) Genetica Medica, Università di Pavia et Fondazione IRCCS S. Matteo, Pavia ; (4) Laboratoire d'Oncogénétique et d'Angiogénétique Moléculaire, Hôpital Pité-Salpêtrière. Paris ; (5) Centre d'Études Démographiques, Université Lumière Lyon 2 ; (6) Service de Génétique, Hôtel-Dieu, Hospices Civils de Lyon; (7) Gastoenterologia ed Endoscopia Digestiva, Ospedale Maggiore, Crema

La maladie de Rendu-Osler est une maladie autosomique dominante caractérisée par des malformations artérioveineuses. Elle est principalement causée par les mutations germinales de deux gènes, ENG et ACVRLI. Il y a une vingtaine d'années, une étude épidémiologique effectuée en France a montré que la prévalence de la maladie était largement sous évaluée (actuellement considérée comme supérieure à $1 / 8000$ ) et qu'elle pouvait beaucoup varier d'une région à l'autre. Deux concentrations de patients ressortaient de façon évidente : la première était située dans la région du Haut-Jura et la deuxième, de moindre importance, dans le département des Deux-Sèvres. Bien que les mutations associées à la maladie de Rendu-Osler soient majoritairement des mutations privées, certaines d'entre elles ont été rapportées à plusieurs reprises, parfois dans des pays différents, surtout dans le cas du gène $A C V R L 1$. Nous avons étudié les haplotypes de 116 patients vivant en France ou dans le Nord de l'Italie et porteurs de 13 mutations différentes du gène ACVRLI, à l'aide de 10 microsatellites couvrant une région de 20 mégabases. Pour cinq mutations, nous avons pu estimer l'âge de l'ancêtre commun le plus récent à l'aide du programme ESTIAGE. Nous avons également mis en relation nos données génétiques avec les données de l'étude épidémiologique antérieure. Nos résultats montrent que la plupart des mutations étudiées résultent vraisemblablement à la fois d'évènements mutationnels indépendants et d'un effet fondateur. L'âge de l'ancêtre commun le plus récent variait entre 100 et 550 ans, selon la mutation. La mutation c.1112dupG, qui a été exclusivement rapportée chez des patients d'origine française, est responsable de la grande concentration de familles qui a été mise en évidence dans les montagnes du Haut-Jura au cours de l'étude épidémiologique. Cette mutation serait survenue chez un habitant de la vallée de la Valserine il y a environ 325 ans puis sa fréquence s'y serait accrue du fait de la dérive génétique. À partir de la fin du $\mathrm{XIX}^{*}$ siècle et au cours du $\mathrm{XX}^{*}$ siècle, elle s'est répandue dans la région environnante, particulièrement dans les centres urbains locaux et régionaux. De façon originale, cette mutation constitue donc un marqueur biologique de la migration d'une population rurale. Inversement, la mutation p.Arg374Gln est survenue indépendamment dans plusieurs aires géographiques différentes. Dans le cas de l'haplotype porté par les familles du département des Deux-Sèvres, l'âge de l'ancêtre commun le plus récent à été estimé à environ 100 ans (4 générations) ce qui est compatible avec une distribution des familles plus concentrée que dans le cas du Haut-Jura. Parmi les 5 mutations communes aux patients français et italiens, deux d'entre elles étaient associées à des haplotypes différents. Pour les 3 autres, et en particulier dans le cas de la mutation p.Arg144X, certains patients des deux pays partageaient un haplotype commun plus ou moins étendu. Compte tenu de l'histoire des migrations récentes, il est vraisemblable que certaines de ces mutations soient survenues chez un ancêtre commun vivant en Italie, puis aient été introduites en France ultérieurement par certains de ses descendants.

Mots-clés : ACVRLI, Rendu-Osler, effet fondateur.

\section{DP23 / 73. IDENTIFICATION DE GRANDS REMANIEMENTS DANS LE GENE $M V K$ PAR QF-PCR}

S. Soler, C. Rittore, L. Philibert, S. Grandemange et I. Touitou

Unité médicale des maladies auto-inflammatoires, $\mathrm{CHU}$ A.-de-Villeneuve Montpellier

Le déficit en mévalonate kinase (MKD) est une maladie auto-inflammatoire héréditaire caractérisée par des épisodes récurrents de fièvre et d'inflammation. Des mutations dans le gène codant la mevalonate kinase $(M V K)$ sont responsables de MKD. Actuellement, nous utilisons dans notre laboratoire, des techniques classiques nous permettant de détecter uniquement des mutations ponctuelles. Il nous a paru intéressant de développer pour ce gène, une technique identifiant de plus larges réarrangements tels que des délétions ou des duplications d'exons. C'est pourquoi, nous avons mis au point la technique de Quantitative Fluorescent-PCR (QF-PCR) pour l'étude de l'ensemble des 10 exons du gène $M V K$ en adaptant la technique décrite par Casilli et al. L'analyse par QF-PCR d'une cohorte de 51 témoins, a permis de détecter pour 20 d'entre eux, une duplication de l'exon 11 qui semble être un polymorphisme de nombre de copie (copy number polymorphism, CNP). Cette technique de PCR multiplex, sensible et reproductible, va être utilisée afin d'identifier de possibles grands remaniements chez certains patients, dont le diagnostic clinique MKD a été confirmé, alors qu'aucune mutation ponctuelle du gène n'a été trouvée.

Mots-clés : mevalonate kinase, QF-PCR, PCR multiplex. 
IP324 / 75. HYPERGLYCINÉMIE SANS CÉTOSE : APPORT DE LA MLPA À L'IDENTIFICATION DES DÉLÉTIONS DANS LE GÈNE GLDC

C. Acquaviva, M.-O. Rolland, S. Ruet, I. Maire, R. Froissart

Service des Maladies Héréditaires du Métabolisme, Centre de Biologie Est, Hospices Civils de Lyon, 59 Bd Pinel, 69500 Bron

Introduction : L'hyperglycinémie sans cétose (NKH), transmise selon le mode autosomique récessif, est due à un déficit de l'un des quatre composants du complexe clivant la glycine (CCG). La forme la plus classique débute en période néonatale et son pronostic est sévère. Le diagnostic biochimique repose sur la mise en évidence d'une élévation majeure et isolée de la glycine à la fois dans le sang et le LCR. Le diagnostic est confirmé par la mise en évidence du déficit enzymatique dans une biopsie de foie. Il doit être complété par l'identification de mutations dans un des gènes codant les protéines du CCG. Parmi ces protéines, la protéine $\mathrm{P}$ codée par le gène $G L D C$, est déficitaire dans plus de $80 \%$ des cas de NKH. Le gène GLDC localisé sur le chromosome 9 (en 9p24) comprend 25 exons. La plupart des mutations identifiées sont privées. Matériel et méthodes : L'étude mutationnelle du gène $G L D C$ a été réalisée en première intention par séquençage direct des exons et des jonctions intron-exon chez 56 patients. Dans certains cas, la recherche de délétion a ensuite été effectuée en utilisant le kit MLPA (Multiplex Ligation dependent Probe Amplification) (Salsa MLPA kit P209 GLDC, MRC-Holland). Résultats et discussion: L'étude de première intention a permis de déterminer le génotype complet de 43 patients : 38 avec des altérations de petite taille et 5 avec une délétion d'un ou plusieurs exons à l'état homozygote. Pour les 11 patients restants, 1 seul allèle a été identifié. L'utilisation de la technique MLPA a alors permis de compléter le génotype de ces patients par la mise en évidence de délétions de taille variable (emportant 1 à 25 exons) à l'état hétérozygote. Cette technique a d'autre part confirmé la présence des 5 délétions à l'état homozygote. Au total, le génotype complet a donc été établi pour tous les patients. Des délétions de taille variable dans le gène $G L D C$ ont été identifiées chez 16 patients soit une fréquence allélique de $19 \%$. Conclusion: Ce travail confirme la fréquence importante des délétions dans le gène $G L D C$ et l'intérêt de l'utilisation de la MLPA pour leur identification. Le diagnostic prénatal de l'hyperglycinémie sans cétose par la technique enzymatique étant souvent d'interprétation délicate, il est indispensable d'avoir les outils pour identifier les mutations des patients et de pouvoir ainsi proposer un diagnostic prénatal fiable aux couples à risque.

Mots-clés : NKH, délétions, autosomique recessif.

\section{"P325 / 77. DÉTECTION DE MICRODUPLICATIONS PAR MLPA CHEZ DES SUJETS TUNISIENS PRÉSENTANT UN RETARD MENTAL INEXPLIQUÉ}

L. Hila (1), H. Tebourbi (1), L. Ben Jemaa (2), F. Maazoul (2), R. M'rad, H. Chaabouni (2)

(1) laboratoire de génétique humaine-Faculté de médecine-tunis : (2) Service des maladies congénitales et héréditaires-EPS Charles Nicole-Tunis Introduction :La prévalence du retard mental dans la population générale est estimée à $3 \%$. Des anomalies chromosomiques sont retrouvées dans environ $10 \%$ des cas et constituent la première cause génétique du retard mental. Cependant pour $60 \%$ des patients aucune étiologie n'est retrouvée. Grâce à la cytogénétique moléculaire il a été démontré qu'environ $5 \%$ des retards mentaux sont dus à des remaniements cryptiques. Matériel et méthodes : Nous avons testé 25 sujets tunisiens présentant un retard mental idiopathique avec signes dysmorphiques et 8 témoins avec deux kits commerciaux P036B - P070 (MRC-Holland.Amsterdam.Netherlands). Les critères d'inclusion ont été un retard mental inexpliqué, un caryotype normal à un ordre de résolution de 500 bandes et l'exclusion du diagnostic du syndrome X Fragile. Résultats : Les résultats ont été interprétés par comparaison à des témoins normaux et/ou présentant une anomalie connue. L'analyse des données a été basée sur le calcul du rapport de la hauteur/aire d'un pic donné, divisée par la somme des hauteurs/aires de tous les pics pour un individu sur la hauteur/aire du même pic, divisée par la somme des hauteurs/aires de tous les pics du témoin. Nous avons mis en évidence des anomalies à type de duplications pures avec 5 cas de dup $4 \mathrm{q}$ et deux duplications dup 15q plus dup Xq retrouvées chez trois sœurs. Discussion : L'existence de séquences recombinogènes localisées aux frontières des remaniements et en rapport avec des recombinaisons homologues non alléliques est connue. Ce mécanisme devrait être à l'origine aussi bien de délétions que de duplications. Cependant peu de syndromes microduplicationnels sont connus à ce jour. Cette différence semble être due à un biais technique, en effet, la variabilité phénotypique des remaniements cryptiques et en particulier des duplications nécessite un criblage indépendant de la symptomatologie clinique. La MLPA (Multiplex Ligation - dependent Probe Amplification) est une technique récente pour l'exploration des extrémités télomériques, riches en séquences répétées, a la recherche de variations du nombre de copies au niveau de ces régions. Elle présente l'avantage de détecter les délétions et duplications partielles qui ne peuvent être décelées par la technique de FISH. Conclusion : La technique MLPA est une technique efficace, simple, rapide, à coût faible et pertinente pour un premier screening des remaniements télomériques.

Mots-clés : MLPA, retard mental, remaniement télomérique.

EP326 / 78. LA DYNAMIQUE MOLÉCULAIRE : UN OUTIL POUR L'INTERPRÉTATION DES CONSÉQUENCES DES MUTATIONS E. Cadet (1), C. Cézard (2), K. Foulon (1), F.Y. Dupradeau (2), J. Rochette $(1,2)$

(1) Laboratoire de Génétique \& INSERM 0351, CHU Amiens, France 2, UPJV, UFR-Pôle Santé Amiens

Introduction : Dans le génome des eucaryotes supérieurs, le taux de mutations est estimé à environ $8.5 \times 10-9$ par génération. Le taux de mutations délétères est mathématiquement dérivé du taux de mutations précédent et il est estimé à 1.2 par génome haploïde et par génération (Hague-Liautard et al., 2007). Un nombre important de mutations est donc sans effet notable. Les conséquences fonctionnelles des mutations dépendent uniquement de leur nature et de leur localisation. Lorsqu'il s'agit de mutations présentes dans les séquences codantes, l'effet sur la protéine n'est pas nécessairement évident, même lorsqu'il existe une nouvelle différence de charge, de taille ou de polarité. Des effets de compensation par de nouvelles interactions peuvent conserver les structures, si ce n'est tout à fait intactes, au moins suffisantes pour ne pas entraîner de conséquences pathoglogiques notables (Dupradeau et al., 2000). Matériel et Méthodes: En l'absence de tests fonctionnels parfois difficiles à réaliser, il existe une alternative de plus en plus utilisée : la modélisation moléculaire. Trois principaux outils existent à l'heure actuelle : i) la modélisation par homologie (logiciel What if), ii) la modélisation en solvant implicite (logiciel Amber 8), iii) la modélisation en solvant explicite (autres programmes Amber). Nous avons utilisé les deux premières techniques dans le but d'interpréter un certain nombre de mutations déjà décrites ou nouvelles, liées à des gènes impliqués dans l'hémochromatose. Leurs applications sont décrites. Résultats : La modélisation par homologie, simple et rapide donne des résultats statiques et très approximatifs. Cette technique est exemplifiée par la construction de modèles originaux mutés du gène HFE (Hémochgromatose de type 1, mutation Q283P, C282Y) et de celui qui code la ferroportine (G490D). Nous en soulignons les limites. Les deux dernières techniques beaucoup plus résolutives donnent des résultats dynamiques et permettent par exemple d'illustrer les conséquences des mutations non seulement sur les structures $3 \mathrm{D}$ mais aussi renseignent sur les interactions avec un ligand. En particulier, nous montrons que notre stratégie permet de renseigner sur l'interprétation des conséquences de deux mutations en cis, traitées séparément et conjointement (HFE : Gly43Asp+His63Asp) par rapport à la structure sauvage. Cet allèle complexe a été découvert, hérité avec le second allèle porteur de la mutation Cys282Tyr. Nous montrons également les conséquences de mutations du gène HFE sur les interactions avec le récepteur 1 de la transferrine (TfR1). Nous avons ainsi mis en évidence le rôle crucial du résidu G43 dans une interaction avec le récepteur de la transferrine. Sa substitution par un aspartate entraîne la perte de flexibilité d'une boucle liant le domaine alpha-1 au domaine alpha-2 de la protéine HFE interagissant avec TfR1. Nous démontrons l'absence d'effet de la mutation His63Asp sur la structure 3D de la protéine HFE et l'absence de conséquence dans sa liaison avec le TfR1 (Dupradeau et al. sous presse). Conclusion : L'innocuité de la mutation His63Asp est une indication pour le séquençage de la totalité du gène HFE chez les homozygotes His63Asp surchargés en fer, voire chez certains C282Y/H63D dont le phénotype de présentation de la surchage en fer est le même que celui des patients Cys282Tyr homozygotes. La recherche de mutations dans d'autres gènes impliqués dans le métabolisme du fer peut être aussi envisagé dans ces cas précis. Notre approche de modélisation moléculaire constitue une aide à la décision du séquençage, elle peut bénéficier à de nombreux généticiens, la seule limite étant la puissance des outils de calcul disponibles dans les laboratoires.

Mots-clés : Mutation, modélisation moléculaire, hémochromatose.

EP327 / 79. CONSÉQUENCES MOLÉCULAIRES D'UNE DÉLÉTION INTRONIQUE DU GENE WNKI RESPONSABLE D'HYPERTENSION HYPERKALIEMIQUE FAMILIALE (HHF) : MISE EN ÉVIDENCE D'ÉLÉMENTS RÉGULATEURS

E. Elvira-Matelot, C. Delaloy, A.M. Houot, X. Jeunemaitre, J. Hadchouel Unité INSERM U772, Collège de France 
L'étude génétique de l'HHF, une forme rare autosomique dominante d'hypertension artérielle, a révélé un nouveau mécanisme de régulation de la pression artérielle grâce à l'identification des gènes WNKI et WNK4 (With No lysine (K) kinase let 4). Ces kinases, exprimées notamment dans le tubule distal rénal, jouent un rôle complexe dans la régulation du transport ionique $(\mathrm{Na}+, \mathrm{K}+, \mathrm{Cl}-)$. Le gène $W N K I$ est à l'origine de plusieurs isoformes : des isoformes longues (L-WNK1) ubiquitaires issues de deux promoteurs proximaux $\mathrm{P} 1$ et $\mathrm{P} 2$, et des isoformes courtes rénales (KSWNK1), issues d'un promoteur rénal rP. Les mutations observées dans l'HHF sont de larges délétions au niveau de l'intron 1, et des résultats préliminaires du laboratoire montrent que cette délétion a pour conséquences une surexpression généralisée de L- et de KS-WNK1 ainsi qu'une expression ectopique généralisée de KS-WNK1. Ces données suggèrent la présence dans l'intron 1 d'un ou plusieurs éléments répresseurs et/ou insulateurs. L'objectif de l'étude a donc consisté à rechercher et caractériser ces différents éléments régulateurs. Cinq séquences candidates (C1-5), toutes localisées dans la délétion minimale $(22 \mathrm{~Kb})$ observée chez les patients HHF, ont été identifiées par recherche bioinformatique de séquences conservées entre espèces. Après clonage de $\mathrm{C} 1$ à $\mathrm{C} 5$ en aval des trois promoteurs de $W N K I$, la recherche d'un effet répresseur in vitro a été effectuée en mesurant l'activité luciferase après transfection transitoire dans trois types cellulaires. La recherche d'une séquence insulateur a été effectuée après insertion de $\mathrm{Cl}$ à $\mathrm{C} 5$ de part et d'autre de l'enhancer rénal en amont du promoteur $\mathrm{rP}$. Les résultats suggèrent que la séquence C4 pourrait jouer le rôle d'un élément répresseur spécifique du promoteur $\mathrm{P} 2$; de même en ce qui concerne la séquence $\mathrm{Cl}$ vis-à-vis du promoteur rP. Ceci pourrait expliquer en partie la surexpression des isoformes de WNK1 observées après délétion de l'intron 1. La séquence C5 bloque l'activité de l'enhancer rénal et pourrait jouer le rôle d'un insulateur. Ceci est aussi cohérent avec l'expression ectopique de KS-WNK1 chez la souris transgénique. Si confirmés, ces résultats seraient en faveur d'un mécanisme transcriptionnel complexe à l'origine de l'HHF qui serait lié à la suppression d'au moins deux répresseurs et un insulateur.

Mots-clés : hypertension hyperkaliémique familiale, WNK1, répresseurs, insulateurs.

\section{EP328 / 84. ANALYSE MOLÉCULAIRE DU GÈNE CUL7 DANS UNE SÉRIE DE 58 PATIENTS PORTEURS D'UN SYNDROME 3 M}

C. Huber (1), A.-L. Delezoide (2), C. Bauman (2), V. Malan (1), A. Munnich (1), V. Cormier-Daire (1) et le groupe collaboratif sur le syndrome 3 $\mathrm{M}$

(1) Department of Genetics and INSERM U781, Hôpital Necker-Enfants Malades, Paris, France; (2) Department of Genetics, Hôpital RobertDebré, Paris, France

Le syndrome 3 M (OMIM 273750) est une maladie autosomique récessive, caractérisée par un retard de croissance pré et post-natal, une dysmorphie faciale, une macrocéphalie relative, une intelligence et un bilan endocrinien normaux. Au cours du développement, des manifestations osseuses apparaissent telles qu'un aspect grêle des os longs, une augmentation de la hauteur des vertèbres et un retard d'âge osseux. L'étude d'une série de 29 familles nous a permis de localiser le locus de la maladie sur le chromosome $6 \mathrm{p} 21.1$ puis d'identifier le gène $C U L 7$ comme responsable du syndrome $3 \mathrm{M}$. A la suite de cette étude, nous avons pu collecter les prélèvements de 29 nouvelles familles porteuses d'un syndrome $3 \mathrm{M}$, et identifier des mutations dans 16 familles sur 29 comprenant 18 nouvelles mutations. Au sein de cette série, le diagnostic de syndrome $3 \mathrm{M}$ avait été évoqué chez un fæutus interrompu à $33 \mathrm{SA}$, présentant un retard de croissance sévère, un périmètre crânien normal, des caractéristiques faciales (lèvres épaisses, nez court, narines antévertées, front haut), des talons proéminents et des os longs grêles. Par séquençage direct, nous avons identifié une mutation située sur un site d'épissage (c.1215+1G $>\mathrm{A})$, héritée du père. De plus, nous avons mis en évidence une absence de contribution maternelle et une complète isodisomie du chromosome 6 , d'origine paternelle. L'étude histologique de la plaque de croissance du fémur de ce fæetus montre une augmentation de la densité et de la taille des chondrocytes localisés dans les zones de réserve et de prolifération. Aucune anomalie majeure des zones préhypertrophique et hypertrophique n'a été notée, suggérant que $C U L 7$ est principalement impliqué dans la prolifération des chondrocytes mais pas dans leur différentiation. Nous concluons que $C U L 7$ est le gène majeur à l'origine du syndrome $3 \mathrm{M}$ avec des mutations identifiées dans $77,6 \%$ des cas (45/58). L'absence de mutation chez 13 de nos patients et l'exclusion du locus $6 \mathrm{p} 21.1$ dans 5 familles consanguines est en faveur d'une hétérogénéité génétique du syndrome $3 \mathrm{M}$.

Mots-clés : syndrome $3 \mathrm{M}, C U L 7$, isodisomie uniparentale chromosome 6.

\section{שP329 / 87. IDENTIFICATION D'UN NOUVEAU LOCUS DE} DÉPLÉTION DE L'ADN MITOCHONDRIAL

J. Mollet (1), J.-P. Jais (2), E. Sarzi (1), D. Chretien (1), A. Munnich (1), A. Rötig (1)

(1) INSERM U781, Hôpital Necker-Enfants Malades, 149 rue de Sèvres, 75015 Paris, France; (2) SBIM, Hôpital Necker-Enfants Malades, 149 rue de Sèvres, 75015 Paris, France

Les déplétions de l'ADN mitochondrial (ADNmt) sont caractérisées par la réduction du nombre de copies de l'ADNmt responsable d'un déficit multiple des enzymes de la chaîne respiratoire mitochondriale. Elles représentent un groupe de patients cliniquement et génétiquement hétérogène. Ces déplétions résultent de mutations de gènes nucléaires impliqués dans le maintien de l'ADNmt. Dans notre série de plus de 100 patients avec déplétion sévère de l'ADNmt, la mutation provoquant la maladie a été identifiée chez seulement $30 \%$ d'entre eux. Nous avons identifié une grande famille consanguine d'Afrique du Nord avec un déficit multiple de la châne respiratoire associé à une déplétion de l'ADNmt. Les quatre patients ont présenté une hypotonie néonatale, un retard de croissance et/ou une hépatomégalie. Les bilans métaboliques ont révélé une hyperlactatémie et/ou un taux élevé de thymidine et d'uracile dans les urines, mais ces caractéristiques n'ont pas été systématiquement trouvées pour tous les patients. Les analyses biochimiques du muscle squelettique effectuées sur trois des patients ont révélé un déficit multiple de la chaîne respiratoire. Un tour de génome (SNP 10K Affymetrix) a été réalisé, mais n'a pas permis d'identifier une région d'homozygotie commune aux quatre enfants atteints. Une étude plus fine des données cliniques, biologiques et enzymologiques a soulevé l'hypothèse de l'existence de deux maladies dans cette famille. En partant de cette hypothèse, nous avons identifié une première région d'homozygotie sur le chromosome 1 contenant le gène $D P Y D$ codant pour la dihydropyrimidine déshydrogenase. Une mutation homozygote de DPYD a été trouvée chez les trois patients ayant un taux élevé de thymidine et d'uracile dans les urines. Une deuxième région de $21 \mathrm{Mb}$, commune aux deux patients présentant des taux élevés de lactate et donc a priori une maladie mitochondriale, a été identifiée sur le chromosome 6. Les gènes candidats de cette région sont actuellement en cours d'analyse. Ce travail permettra l'identification d'un nouveau gène nucléaire responsable de déplétion de l'ADNmt.

Mots-clés : mitochondrie, déplétion, ADNmt.

-P330 / 88. UNE NOUVELLE MUTATION DÉCALANTE DU GÈNE FGA (CHAÎNE ALPHA DU FIBRINOGÈNE) INDUIT L'APPARITION D'UN MOTIF PEPTIDIQUE VLITL TRES AMYLOÏDOGËNE CONDUISANT À UNE AMYLOSE RÉNALE

S. Valleix (1), P. Le Pogamp (2), P. Derreumaux (3), C. Vasseur (1), G. Grateau (1), M. Delpech (1)

(1) Service de Biochimie et Génétique Moléculaire et U567-CHU CochinParis; (2) Service de Néphrologie-CHU de Rennes; (3) Laboratoire de Biochimie théorique et Institut de Biologie Physico-Chimique-UPR9080 CNRS et Université Paris VII

Une nouvelle mutation décalante du gène $F G A$ (chaîne alpha du fibrinogène) induit l'apparition d'un motif peptidique VLITL très amyloïdogène conduisant à une amylose rénale. Les amyloses héréditaires liées à la chaîne alpha du fibrinogène sont des entités très rares, à tropisme rénal, qui se manifestent vers la $4^{\circ}$ décennie par une protéinurie et évoluent inéluctablement vers l'insuffisance rénale chronique terminale. La transmission de cette maladie est autosomique dominante avec une pénétrance variable expliquant l'absence fréquente d'histoire familiale. Le pronostic rénal défavorable nécessite, dans les cinq ans suivant le diagnostic, une transplantation rénale qui elle-même se solde rapidement par un échec dû à la récidive des dépôts amyloïdes. Sur le plan moléculaire, on distingue deux types de mutations, les mutations fauxsens et les mutations décalantes, mais toutes ces mutations amylö̈dogènes surviennent exclusivement au niveau de l'exon 5 du gène FGA codant la partie C-terminale de la chaîne alpha. Les formes les plus sévères d'amylose rénale sont celles liées aux mutations décalantes. Trois mutations décalantes « privées » ont été décrites, chacune d'entre elles n'ayant été rapportée que dans une seule famille. Ces trois mutations décalantes conduisent, toutes, à un arrêt prématuré de la chaîne alpha du fibrinogène au niveau du codon 548 au lieu du codon 610, produisant une chaîne alpha avec une partie C-terminale plus courte et dont la séquence des derniers 27 acides aminés est modifiée. Ici, nous rapportons une nouvelle famille française atteinte d'amylose rénale et chez laquelle nous avons identifié une nouvelle mutation décalante dans le gène $F G A$, conduisant à la synthèse d'une chaîne alpha mutante dont la séquence $\mathrm{C}$-terminale est similaire à celles résultantes des trois autres mutations décalantes rapportées jusque-là. Les mécanismes à l'origine de la fibrillogénèse de la chaîne alpha du fibrinogène restent totalement inconnus, et à la suite de cette nouvelle famille, nous avons émis l'hypothèse que la partie 
C-terminale mutante résultant de cette nouvelle mutation décalante pouvait contenir une séquence peptidique particulière responsable du caractère amyloïdogène de FGA. Cette hypothèse était également fondée sur le fait que seuls des fragments protéolysés contenant la partie $\mathrm{C}$-terminale mutée de la chaîne alpha du fibrinogène sont retrouvés dans les fibrilles amyloïdes. À cette fin, nous avons utilisé différents logiciels informatiques, notamment les algorythmes TANGO et CSSP, qui ont précédemment détecté avec succès les régions amyloïdogènes de nombreuses protéines amylö̈des comme le prion, le lyzosyme, la transthyrétine et le peptide A-béta de la maladie d'Alzheimer. Les analyses in silico que nous avons menées ont montré que la chaine alpha du fibrinogène sauvage ne présente pas de caractéristique intrinsèque amylö̈dogène et que les différents mutants amylö̈dogènes (mutations faux-sens versus mutations décalantes) ne partagent pas un mécanisme commun d'aggrégation. Pour les mutations décalantes, les résultats indiquent que le peptide de séquence VLITL, présent uniquement dans la partie C-terminale des chaînes alpha dérivant des mutations décalantes et absent dans la chaîne alpha normale est une région importante pour promouvoir l'aggrégation en feuillets béta plissés et qu'il constitue probablement le noyau fibrillogénique. La prédiction que ces 5 résidues hydrophobes VLITL sont aptes à former des structures pouvant favoriser la conformation en feuillets béta plissés est en accord avec l'observation récente que la séquence peptidique VII, très proche de VLI, est capable à elle seule de former, in vitro, des fibrilles amyloïdes. L'identification de ce pentapeptide amyloïdogène pourrait permettre d'envisager une nouvelle voie d'approche thérapeutique pour ces amyloses par l'élaboration de drogues inhibitrices ciblant de façon spécifique la séquence VLITL.

Mots-clés : amylose héréditaire, chaîne alpha du fibrinogène, atteinte rénale.

口P331 / 94. INFERTILITÉ MASCULINE PAR ABSENCE DES CANAUX DÉFÉRENTS : INTÉRÊT DE LA RECHERCHE DE RÉARRANGEMENTS DU GENE CFTR POUR LE DIAGNOSTIC ET LE CONSEIL GÉNÉTIQUE

I. Ratbi (1), M. Legendre (1), F. Niel (1), J. Martin (1), J.-C. Soufir (2), V. Izard (3), B. Costes (1), A. Lefloch (1), C. Costa (1), M. Goossens (1) E. Girodon (1)

(1) Service de Biochimie et Génétique Moléculaire, AP-HP et INSERM U841, Hôpital Henri-Mondor, 94010 Créteil, France ; (2) Service de Biologie de la Reproduction et du développement, AP-HP, Hôpital de Bicêtre, 94270 Le Kremlin-Bicêtre, France; (3) Service d'Urologie, AP-HP, Hôpital de Bicêtre, 94270 Le Kremlin-Bicêtre, France

Introduction: La recherche de mutations du gène de la mucoviscidose (gène CFTR) fait partie du bilan étiologique des infertilités masculines par absence bilatérale des canaux déférents $(\mathrm{ABCD})$. Les patients sont souvent hétérozygotes composites pour une mutation à effet «modéré » et une mutation à effet "sévère ", associée à la mucoviscidose, et cette notion est importante pour le conseil génétique des couples bénéficiant d'une procréation assistée. Cependant, selon les études, au moins une mutation n'est pas identifiée chez 20 à $55 \%$ des patients. La recherche de grands réarrangements du gène CFTR, récemment introduite dans les études génétiques des patients atteints de mucoviscidose, a été effectuée dans une cohorte de patients infertiles par $\mathrm{ABCD}$ au phénotype bien caractérisé, ce qui a permis de faire le point sur l'ensemble des génotypes associés à cette pathologie. Méthodes: L'exploration du gène CFTR chez 222 patients infertiles par $\mathrm{ABCD}$ a reposé sur une combinaison de méthodes de balayage (DGGE, DHPLC et séquençage) et, pour une sélection de 61 patients, sur une technique d'amplification multiplex semi-quantitative de l'ensemble des régions codantes, à la recherche de grandes délétions, duplications et insertions. Résultats : Près de $88 \%$ des allèles ont ainsi été caractérisés et $82 \%$ des patients sont porteurs de deux mutations. Les 10 mutations les plus fréquentes rendent compte de $74.6 \%$ des allèles identifiés. Quatre grands réarrangements ont été identifiés chez des patients porteurs d'une mutation modérée: deux délétions partielles déjà rapportées (emportant les exons $17 \mathrm{a}$ à 18 et 22 à 23 ), une délétion complète et une nouvelle duplication des exons 11 à 13. Ces réarrangements rendent compte de $7 \%$ des allèles précédemment non identifiés et de $1 \%$ du total des allèles. Conclusion : Ces résultats illustrent l'intérêt pour le diagnostic et le conseil génétique d'une étude complète du gène CFTR chez les patients infertiles par $\mathrm{ABCD}$, incluant la recherche de grands réarrangements.

Mots-clés : mucoviscidose, CFTR, infertilité masculine.

-P332 / 96. ÉTAT DES LIEUX DES SURDITÉS GÉNÉTIQUES EN ALGÉRIE

C. Giannesini (1), F. Ammar-Khodja (2), V. Faugère (1), S. Léonard (1), M. Makrelouf (3), R. Malek (4), M. Djenaoui (5), A. Zenati (3), M. Claustres $(1,6,7)$, A-F Roux $(1,6)$
(1) CHU Montpellier, Laboratoire de Génétique Moléculaire, Montpellier ; (2) Department of Molecular and Cell Biology, Biological Faculty of Sciences, University of USTHB, El Alia, Bab-Ezzouar, Alger, Algérie; (3) Genetic Unit of Biochemistry, Central Laboratory, CHU Bab-El-Oued, Alger, Algérie ; (4) Department of Otolaryngology, CHU Bab El Oued, Alger, Algérie ; (5) Department of Otolaryngology, CHU Mustapha, Alger, Algérie; (6) Inserm, U827, Montpellier; (7) Univ, Montpellier I, Montpellier

Afin d'identifier les origines génétiques des surdités en Algérie, 54 familles d'enfants scolarisés dans des écoles spécialisées pour malentendants ont été prélevées et analysées à différents loci connus comme étant impliqués dans les surdités non syndromiques (DFN). L'origine génétique de la surdité était supposée par la présence de plusieurs membres atteints au sein de chaque fratrie. Les enfants scolarisés ont pu bénéficier d'un suivi clinique et des audiogrammes ont permis d'évaluer précisément leur degré de déficience auditive. Tous les patients présentaient une surdité congénitale moyenne à profonde. Deux enfants présentaient par ailleurs des troubles de la vision nocturne pouvant laisser suggérer un syndrome de Usher. Une consanguinité était connue dans $50 \%$ des familles étudiées. Le locus DFNB1 se trouve majoritairement impliqué dans les surdités congénitales non syndromiques. Dans un premier temps, l'étude moléculaire exhaustive de ce locus, par analyse des exons codant et non codant et des délétions GJB6-D13S1830 et GJB6-D13S1854, a été réalisée sur l'ADN des 54 cas index. Vingt patients ont été génotypés avec des mutations délétères établissant l'implication du locus DFNBI à $37 \%$. Parmi les patients DFNB1, $70 \%$ portent la mutation $35 \mathrm{delG}$ à l'état homozygote. Cependant l'identification de 5 autres mutations du gène GJB2 (E47X, V37I, c.167delT, $\mathrm{N} 206 \mathrm{~S}$ et IVS1+1G $>\mathrm{A}$ ) montre qu'une étude restreinte à la mutation majoritaire n'est pas satisfaisante. Chez trente-quatre cas index, aucun génotype $D F N B 1$ pathogène n'a pu être identifié. L'ADN des apparentés étant disponible pour 16 d'entre eux, une analyse des haplotypes DFNB1 a alors été effectuée dans le but d'exclure définitivement son implication. Ce locus a été exclu pour 10/16 familles analysées; l'hypothèse d'une mutation $D F N B 1$ Algérienne commune à toutes ces familles a pu être éliminée. Le laboratoire de Montpellier est par ailleurs spécialisé dans le diagnostic moléculaire du syndrome de Usher (analyse exhaustive de 8 gènes Usher). Certains des loci USH sont également impliqués dans les surdités non syndromiques: DFNB2/USH1B (MYO7A), DFNB12/USH1D (CDH23), DFNB23/USH1F (PCDHI5), DFNB18/USH1C (USHIC), USH1G (USHIG) et DFNB31/USH2D (WHRN). Une étude de l'ensemble de ces loci a été effectuée sur la cohorte. L'efficacité de cette approche est renforcée par un taux de consanguinité élevé au sein des familles Algériennes et une homozygotie à un locus donné peut alors être recherchée. Le séquençage du gène correspondant a permis d'identifier les mutations pathogènes dans les gènes MYO7A ( 2 familles) et $C D H 23$ ( 1 famille). La même démarche appliquée aux 2 patients suspectés d'un syndrome de Usher a conduit à la caractérisation de 2 mutations délétères, dans $M Y O 7 \mathrm{~A}$ et $P C D H 15$, confirmant ainsi le diagnostic clinique chez ces deux enfants. Cette étude démontre la très forte hétérogénéité génétique de la population algérienne qui rend complexe toute mise en place d'un diagnostic moléculaire efficace. Une des causes majeures des surdités congénitales reste le taux très important des mariages consanguins, ce qui constitue un véritable problème de santé publique.

Mots-clés : surdité, génotype, haplotype.

\section{aP333 / 98. MOSAÏQUES SOMATIQUES MASCULINES : EST-CE SI RARE EN HÉMOPHILIE ?}

C. Costa (1, 2), A.M. Frances (3), S. Letourneau (1), P. Conteville (1), E. Girodon-Boulandet $(1,2)$, M. Goossens $(1,2)$

(1) AP-HP, Groupe Henri Mondor-Albert Chenevier, Laboratoire de Génétique Moléculaire ; (2) INSERM U841, Département de Génétique, équipe 11, Université Paris 12, Créteil F-94000 ; (3) Hôpital Font-Pré, Génétique Médicale, Toulon, 83000 France

Environ $1 / 3$ des cas d'hémophilie sont sporadiques, dont $20 \%$ sont dus à une néo mutation survenue soit au niveau germinal soit très tôt durant l'embryogenèse, ainsi à l'origine de mosaïques germinale ou somatique. En hémophilie, la plupart des cas de mosaïque somatique ont été rapportés chez des femmes conductrices. Seuls trois cas ont été rapportés chez des hommes dans la littérature. Nous présentons ici un cas exceptionnel de mosaïque somatique chez le grand-père asymptomatique d'un hémophile A sévère. La mutation délétère identifiée par séquençage direct chez le propositus est également retrouvée chez la mère et la tante maternelle de celui-ci, suggérant une grand-mère conductrice. La mutation n'étant retrouvée ni dans les leucocytes ni dans les cellules buccales et uro-épithéliales de cette dernière, cette hypothèse est remise en cause et soulève la question de l'origine de l'anomalie. L'étude de la ségrégation des 
marqueurs liés au gène du facteur VIII permet d'identifier l'origine de l'allèle délétère chez le grand père paternel asymptomatique. Parce que les mosaïques somatiques peuvent être difficiles à détecter par les méthodes conventionnelles, elles nécessitent des procédures d'enrichissement non utilisables en routine. Grâce à l'évolution technologique, de nouvelles méthodes plus sensibles telles la DHPLC (denaturing-high-liquid-pressurechromatography) couplée éventuellement à l'utilisation des nucléases, enzymes reconnaissant les mésappariements, semblent être prometteuses. Ainsi, dans le cas présenté ici, leur utilisation en comparaison au séquençage direct montre chez le grand-père de l'hémophile une meilleure sensibilité à détecter l'allèle muté. Dans les cas sporadiques il est important pour le conseil génétique de déterminer s'il existe un risque familial. Un résultat négatif n'élimine pas la possibilité d'une mosaïque et peut conduire à rassurer faussement sur le statut de conductrice. Ce cas suggère que les mosaïques somatiques sont vraisemblablement sous-estimées, particulièrement dans les cas sporadiques et soulève l'intérêt de réaliser une étude de liaison pour identifier l'origine de l'allèle délétère et d'utiliser des méthodes sensibles pour détecter les mosaïques.

Mots-clés : mosaïque masculine, hémophilie, conseil génétique.

口P334 / 106. ANALYSES MOLÉCULAIRES DU GÈNE LRRK2 CHEZ 233 FAMILLES EUROPÉENNES ET NORD-AFRICAINES ATTEINTES DE LA MALADIE DE PARKINSON

S. Lesage (1), C. Condroyer (1), E. Lohmann (1), A. Lannuzel (4), F. Tison (5), P. Damier (6), A. Dürr (1, 2), A. Brice $(1,2,3)$, et le réseau PDG (1) INSERM U679 Neurologie et Thérapeutique Expérimentale, Hôpital de la Pitié-Salpêtrière, AP-HP, et Université Pierre-et-Marie-Curie, Faculté de Médecine, Paris, France ; (2) Département de Génétique, Cytogénétique et Embryologie, CHU Pitié-Salpêtrière, AP-HP, 75013 Paris, France; (3) Fédération de Neurologie, CHU Pitié-Salpêtrière, AP-HP, 75013 Paris, France; (4) Service de Neurologie, Hôpital Ste-Anne, 75014 Paris, France; (5) Service de Neurologie, Hôpital Haut-Lévêque, 33604 Pessac, France; (6) Service de Neurologie, Hôpital Guillaume-et-RenéLaënnec, 44093 Nantes, France

Introduction : La maladie de Parkinson (MP) est une affection neurodégénérative progressive, caractérisée cliniquement par la triade bradykinésie, rigidité et tremblement de repos, dus à la dégénérescence des neurones dopaminergiques de la voie nigro-striatale. Des mutations dans le gène $L R R K 2$ (Leucine-Rich-Repeat Kinase 2) au locus PARK8 ont été identifiées dans des familles présentant une transmission de type autosomique dominant. Parmi elles, la mutation G2019S (c.6055 G>A) est la plus communément retrouvée, mais avec des fréquences très variables selon l'origine géographique et ethnique des populations étudiées. Du fait que le gène $L R R K 2$ est un grand gène comprenant 51 exons codants, peu d'études ont porté sur le criblage entier du gène dans un grand nombre de familles avec une transmission autosomique dominante. But du travail : Déterminer le spectre mutationnel du gène $L R R K 2$ dans une grande série de familles MP compatibles avec une transmission autosomique dominante et établir des corrélations génotype-phénotype. Patients et methodes: Nous avons sélectionné 233 familles MP compatibles avec une transmission autosomique dominante. Ces familles sont essentiellement d'origine française ( $78 \%$ ), et aussi d'Afrique du Nord $(n=14)$. L'âge moyen de début de la maladie des cas index est de $50,4 \pm 12,3$ ans (extrêmes, 18-86). Les 51 exons du gène $L R R K 2$ et leurs régions flanquantes ont été séquencés dans les 2 sens. RESULTATS : Nous avons identifié 8 mutations potentielles chez 22 cas index $(9,4 \%)$, comprenant 5 substitutions nouvellement identifiées (S52F, D334K, N363S, I810V, R1325Q). La mutation G2019S a été identifiée chez 13 cas index $(5,6 \%)$ dont 6 sont d'origine maghrébine $(6 / 14=43 \%)$. Les mutations hétérozygotes connues $\mathrm{R} 1441 \mathrm{H}$ et I1371V ont été retrouvées, chacune dans une famille. La plupart des mutations identifiées dans notre série sont localisées dans les domaines fonctionnels de la protéine. Le phénotype associé à la présence de ces mutations ressemble à celui d'un syndrome parkinsonien typique avec cependant des âges de début de la maladie très variables [53,2 99,6 ans (extrêmes, 35-71)]. CONCLUSION : L'analyse du gène entier de $L R R K 2$ dans une grande série de familles majoritairement françaises atteintes de MP présentant une transmission autosomique dominante a montré que les mutations sont rares, à l'exception de la mutation G2019S. Le tableau clinique des patients porteurs de mutations de LRRK2 est celui d'un Parkinson typique. En terme de diagnostic moléculaire du gène $L R R K 2$, les mutations connues les plus fréquentes seront criblées en priorité.

Mots-clés : maladie de Parkinson, LRRK2, mutations.

IP335 / 110. UN NOUVEAU SYNDROME POLYMALFORMATIF ASSOCIÉ À UNE MUTATION HOMOZYGOTE DU GÈNE FTO

S. Boissel (1), O. Reisch (2), F. Molinari (1), H. Etchevers (1), A. Munnich
(1) INSERM U781 and Département de Génétique, Hôpital Necker-Enfants Malades, Paris; (2) Department of Medical Genetics, Assaf Harofeh, Israel

Nous présentons ici l'étude d'une famille israélienne consanguine et multiplex, dans laquelle 8 enfants atteints présentent un nouveau syndrome polymalformatif à transmission autosomique récessive et caractérisé par un retard psychomoteur sévère, un retard de croissance intra-utérin, un retard de myélinisation, des anomalies cardiaques, une fente palatine, une dysmorphie faciale et une mort prématurée. Les analyses biochimiques et métaboliques, ainsi que le caryotype haute résolution sont normaux. Mettant à profit la stratégie de cartographie par autozygotie en analysant 5 enfants atteints et 15 apparentés sains, nous avons identifié une région unique d'homozygotie de $6,5 \mathrm{Mb}$ en $16 \mathrm{q} 12-\mathrm{q} 13$, entre les marqueurs D16S411 et D16S3140 (D16S3136, Zmax $=4,16$ à theta $=0)$. Celle-ci contient 29 gènes connus. Le séquençage du gène FTO (fat mass and obesity associated), a ensuite permis de caractériser une mutation homozygote faux-sens dans l'exon 5: c.947G >A, p.R316Q. Cette mutation coségrège avec la maladie et n'a pas été retrouvée chez 400 allèles contrôles dont 100 allèles de la même origine ethnique. Chez la souris, Fto est un des gènes affectés par la mutation Fused toes $(\mathrm{Ft})$, une délétion hétérozygote des gènes du cluster des Iroquois B, Fts, Ftm et Fto. Les souris hétérozygotes $\mathrm{Ft}$ présentent une syndactylie des membres antérieurs. Les embryons homozygotes $\mathrm{Ft} / \mathrm{Ft}$ montrent des anomalies de la formation du tube neural et du développement cérébral qui entraînent des malformations craniofaciales sévères ainsi qu'une mort embryonnaire précoce. De façon très surprenante, des études d'association ont récemment montré qu'un variant hétérozygote dans l'intron 1 de FTO prédispose à l'obésité chez l'enfant et l'adulte. Toutefois, le rôle précis de FTO dans cette pathologie reste inconnu. Des études d'expression par RT-PCR sur des ARNs de différents tissus humains nous ont déjà permis de montrer que FTO est exprimé de façon ubiquitaire au cours du développement embryonnaire et à l'âge adulte. Par ailleurs, des expériences d'hybridation in-situ sur des embryons et des fœetus humains sont en cours afin d'établir plus précisément le patron d'expression de FTO. Enfin, des expériences de transfection avec la protéine sauvage ou mutée (p.R316Q) dans des cellules HEK 293T ont démontré que la protéine est localisée dans le noyau et que la mutation n'affecte pas cette localisation. Nos résultats confirment l'efficacité de la cartographie par autozygotie pour identifier de nouveaux gènes responsables de pathologies autosomiques récessives. Ils mettent également en lumière les bases moléculaires d'un nouveau syndrome polymalformatif. Les analyses en cours devraient nous permettre de mieux caractériser la fonction de FTO au cours du développement embryonnaire et également de comprendre comment un variant hétérozygote peut être associé à l'obésité alors qu'une mutation homozygote du même gène entraîne un syndrome polymalformatif sévère.

Mots-clés : cartographie par autozygotie, syndrome polymalformatif, retard psychomoteur.

IP336 / 114. IDENTIFICATION DE NOUVEAUX TRANSCRITS ALTERNATIFS DU GENE MEFV DANS DES LEUCOCYTES HUMAINS

S. Grandemange (1), C. Notarnicola (2), I. Touitou (1)

(I) Unité médicale des maladies auto-inflammatoires, CHU A.-de-Villeneuve, Montpellier: (2) ERI 25 Muscle et Pathologies, INSERM, CHU A.-de-Villeneuve, Montepllier

Introduction : La fièvre méditerranéenne familiale (FMF) est une maladie autosomique récessive qui se caractérise par des attaques récurrentes de fièvre et inflammation des séreuses. Des mutations dans le gène $M E F V$ sont responsables de la FMF. MEFV, exprimé principalement dans les neutrophiles et les monocytes, code la protéine Marenostrine/Pyrine qui semble être un régulateur de l'inflammation. Récemment, différents transcrits d'épissage alternatifs ont été caractérisés, suggérant l'existence de plusieurs protéines in vivo. Matériels et Méthodes: À ce jour, le profil d'expression de la protéine native n'est pas connu. Dans ce but, nous avons effectué une analyse des messagers MEFV par RT-PCR, à partir d'ARN de leucocytes humains d'individus contrôles. Les produits de PCR, effectuée à l'aide d'oligonucléotides localisés dans les exons 1 et 10 , ont été clonés et directement séquencés. Résultats : Nous avons ainsi identifié des nouveaux messagers issus d'un épissage alternatif. Le transcrit complexe, MEFV-d2-9ext, résulte d'une délétion en phase de l'exon 2 et d'une extension de $117 \mathrm{pb}$ en 5' de l'exon 9, engendrant un codon stop prématuré. Le second transcrit, MEFV-d2-3-4, généré par la délétion des exons 2, 3 et 4, résulte en un décalage du cadre de lecture et prédit une courte protéine correspondant uniquement à la séquence codante de l'exon 1 . Deux isoformes dérivées du MEFV-d2-3-4 présentent en plus, une délétion de l'exon 7 ou des exons 7 et 8 . Conclusion et perspectives: Nos résultats 
confortent les données de la littérature et confirment l'existence d'un nombre important de transcrits MEFV, mais à ce jour, nous ne savons pas quelle(s) variant(s) protéique (s) est (sont) présent(s) dans la cellule. Parce que l'altération de la régulation de l'expression de $M E F V$ pourrait être impliquée dans la physiopathologie de la FMF, nous établirons le profil d'épissage des transcrits MEFV et le profil protéique d'individus contrôles, et nous le comparerons aux profils caractérisés chez des patients atteints de FMF.

Mots-clés : FMF, expression, épissage alternatif.

\section{- 337 / 122. UNE NOUVELLE MUTATION DU GÈNE FRAS1 EN 4Q21CHEZ UN NOURRISSON TUNISIEN ATTEINT D'UN SYN- DROME DE FRASER}

W. Ben Romdhan (4), M. Zenker (2), M. Jallouli (3), R. Louati (1), F. Kallebi (1), R. Mhiri (3), T. Rebai (1), N.B. Abdelmoula (1) (1) Laboratoire d'Histologie, Faculté de Médecine de Sfax Tunisie ; (2) Institut de génétique Humaine, Erlangen Allemagne: (3) Unité de Chirurgie Pédiatrique, CHU Hédi Chaker Sfax Tunisie ; (4) Service de Pédiatrie, CHU Hédi Chaker Sfax Tunisie

Le syndrome de Fraser est un syndrome polymalformatif très rare caractérisé par la présence d'une atteinte oculaire, la cryptophtalmie, d'une syndactylie, d'anomalies génitales, d'une agénésie rénale et de malformations laryngées. Deux gènes du syndrome de Fraser ont été identifiés: le gène FRAS1 en $4 \mathrm{q} 21$ et le gène FREM2 en 13q13. Des mutations ont été identifiées à l'intérieur de FRASI mais rarement au niveau de FREM2. Nous rapportons l'observation clinique d'un nourrisson tunisien de sexe masculin originaire de Gafsa chez qui un syndrome de Fraser a été cliniquement diagnostiqué et pour lequel une étude moléculaire du gène FRAS1 a été pratiquée. Le nourrisson a été hospitalisé à l'âge de deux mois pour cure chirurgicale d'une hernie scrotale droite. Il est issu d'un couple consanguin de second degré ayant déjà un enfant de sexe masculin de 5 ans en bon état de santé. À l'examen clinique, il pesait $4500 \mathrm{~g} \mathrm{(M)}$ et mesurait $50 \mathrm{~cm}(-2 \mathrm{DS})$. Il présentait une dysmorphie faciale avec notamment des anomalies oculaires. Il s'agissait d'une microphtalmie à droite avec fusion des moitiés internes des deux paupières laissant une petite fente palpébrale avec une cornée inaccessible. À l'IRM orbitaire, le globe oculaire était déformé de petite taille avec absence du cristallin et déformation du segment antérieur. Le nerf optique était de morphologie et de localisation normale. Le nourrisson présentait par ailleurs, un hypertélorisme, des anomalies du nez avec une racine large et des ailes hypoplasiques, une agénésie du pavillon de l'oreille droite avec hypoplasie du conduit auditif externe et fusion des deux berges antérieure et postérieure de celui ci à l'exploration tomodensitométrique des rochers. Le nourrisson présentait en outre une syndactylie bilatérale des doigts et des orteils ainsi qu'un stridor audible. L'imagerie par résonance magnétique du cerveau montrait à l'étage sus tentoriel, un discret amincissement du corps calleux et une discrète dilatation des ventricules latéraux. Les organes génitaux externes se caractérisaient par une verge coudée de petite taille avec hypospadias et présence d'une hernie inguinale droite. Les échographies cardiaques et abdominales étaient sans anomalie décelable. Une étude cytogénétique avec marquage des chromosomes en bandes RGH et GTG s'est révélée normale $46, \mathrm{XY}$. Une étude moléculaire indirecte à l'aide de marqueurs microsatellites à proximité des gènes FRAS1 et FREM 2 a permis de montrer une homozygotie pour un large intervalle autour du locus FRAS1. Le séquençage direct de tous les exons et des régions introniques flanquants du gène FRASI a permis de mettre en évidence une mutation homozygote du site d'épissage de l'exon 23 : IVS23+1G $>$ A. Il s'agit d'une nouvelle mutation qui n'a pas été décrite auparavant.

Mots-clés : Fraser syndrome, FRAS1, mutation.

EP338 / 123. RÉDUCTION DE LA RÉGION 2P11.1-Q12.2 RESPONSABLE DU SYNDROME DE TREMBLEMENT MYOCLONIQUE CORTICAL FAMILIAL ASSOCIÉ À UNE ÉPILEPSIE ET EXCLUSION DE GÈNES CANDIDATS

D. Bouteiller (1), C. Saint-Martin (1), G. Stevanin (1, 2), C. Popescu (1), M. Ruberg (1), E. LeGuern (1, 2), P. Labauge (3), C. Depienne $(1,2)$ (1) INSERM U679, Paris ; (2) Département de génétique et cytogénétique, AP-HP, Hôpital Pitié-Salpêtrière, Paris; (3) Service de Neurologie, Hôpital Caremeau, Nîmes

Le tremblement myoclonique cortical familial associé à une épilepsie (TMCFE), également connu sous le nom de FAME (Familial Adult Myoclonic Epilepsy) est un syndrome transmis sur un mode autosomique dominant. Il est caractérisé par un tremblement myoclonique cortical qui débute généralement chez l'adulte et qui peut survenir seul ou être accompagné de crises épileptiques qui sont le plus souvent de type généralisé tonico-clonique. Deux loci, situés dans les régions chromosomiques 8q23.3-q24.11 (FAME1) et 2p11.1-q12.2 (FAME2), ont été précédemment décrits mais aucun gène pathogène n'a été identifié à ce jour. Dans le but d'identifier le locus morbide et le gène en cause dans une large famille d'origine espagnole, nous avons réalisé une cartographie génétique. L'analyse de liaison a montré que la famille est liée à une région située en 2p11-q12 chevauchante avec le locus FAME2. Ce résultat a permis de réduire la région critique à un intervalle de $9,42 \mathrm{~cm}$. Par ailleurs, des mutations dans la séquence codante de cinq gènes candidats contenus dans l'intervalle réduit (KCNIP3, DRDSPI, SIAT9, REEPI et VAMP5) ont été exclues par séquençage direct et par MLPA (multiplex ligation-dependent probe amplification) dans le cas de REEP1. Etant donné qu'aucun gène connu codant pour un canal ionique ou une protéine associée n'est contenu dans l'intervalle réduit, nous proposons des stratégies alternatives pour identifier le gène pathogène de cette région impliqué dans le TMCFE.

Mots-clés : epilepsie, tremblement cortical, liaison génétique.

nP339 / 124. MUTATIONS DE SCN1A HERITÉES ASSOCIÉES À L'ÉPILEPSIE MYOCLONIQUE SÉVÈRE DU NOURRISSON

O. Trouillard (1), C. Saint-Martin (2), I. Gourfinkel-An (2), E. Fedirko (1), R. Nabbout (3), E. LeGuern $(1,2)$, C. Depienne $(1,2)$

(1) Département de génétique et cytogénétique, AP-HP, Hôpital PitiéSalpêtrière, Paris; (2) INSERM U679, Paris ; (3) Service de neuropédiatrie, Hôpital Necker, Paris

Les mutations du gène SCNIA, codant pour le canal sodique neuronal dépendant du voltage Nav1.1, sont associées soit à une épilepsie généralisée avec des convulsions fébriles prolongées (phénotype épileptique variable connu sous le nom de GEFS+) soit à un syndrome beaucoup plus sévère appelé épilepsie myoclonique sévère du nourrisson (EMSN ou syndrome de Dravet). Seules des mutations de type faux-sens, héritées sur un mode autosomique dominant, sont retrouvées dans le GEFS+ alors que la plupart des mutations décrites dans l'EMSN sont des mutations de novo de tous types, en particulier tronquantes ou faux-sens. Cependant, un petit nombre de mutations responsables d'EMSN sont héritées d'un parent asymptomatique ou modérément atteint, soulevant la question d'une expressivité variable de ces mutations et des facteurs déterminant la sévérité de l'épilepsie. L'objectif de ce travail a été de déterminer les mécanismes à l'origine de la variabilité du phénotype chez les parents porteurs d'une mutation associée à une EMSN chez leur(s) enfant(s). Nous avons analysé 11 familles avec un (7 familles) ou deux enfants (4 familles) atteints d'EMSN, pour lesquelles une mutation dans SCNIA héritée d'un parent avait été identifiée. Dans 7 familles, nous avons montré qu'une mutation tronquante était survenue de novo et était à l'état de mosaïque somatique chez le parent transmetteur dans au moins 5 cas sur 7. L'absence d'épilepsie ou la présence d'une épilepsie modérée chez le parent s'explique par le mosaïcisme dans ces familles. Dans 3 familles, une mutation faux-sens était transmise de façon autosomique dominante chez plusieurs membres de la familles présentant un phénotype épileptique de type GEFS + et était également présente chez le patient atteint d'EMSN. Le pronostic clinique chez ces patients était meilleur que l'évolution classique de l'EMSN, bien que celui-ci puisse être aussi observé chez des patients avec des mutations de novo. Nos résultats sont en faveur de deux mécanismes génétiques distincts responsables d'EMSN et de l'existence de facteurs génétiques ou environnementaux additionnels déterminant donc la sévérité du phénotype épileptique. Le mosaïcisme parental pourrait représenter juqu'à $10 \%$ des familles avec au moins un enfant atteint d'EMSN.

Mots-clés : épilepsie, SCNIA, mosaïque.

DP340 / 125. SPECTRE DES MUTATIONS ET CARACTÉRITIQUES CLINIQUES ASSOCIÉES AU GENE SPG4 DANS LES PARAPLÉGIES SPASTIQUES

E. Denis (1), C. Depienne (1, 2), E. Fedirko (1), B. Bricka (1), A. Durr $(1,2)$, A. Brice $(1,2)$

(1) Département de génétique et cytogénétique, AP-HP, Hôpital PitiéSalpêtrière, Paris; (2) INSERM U679, Paris

Les mutations du gène SPAST/SPG4, qui code pour la protéine spastin, représentent la cause la plus fréquente de paraplégie spastique (PS) autosomique dominante pure. Dans le cadre d'un diagnostic moléculaire de routine, nous avons analysé le spectre des mutations de SPG4 et les caractéristiques cliniques présentes chez les patients portant des mutations. Nous avons criblé une large série de 543 patients avec paraplégie spastique pure ou complexe (ie avec des signes cliniques additionnels) par DHPLC (denaturing high performance liquid chromatography), séquençage et MLPA (multiplex ligation-dependent probe amplification). Nous avons identifié 164 familles avec mutations, 133 présentant des substitutions ou des petites 
délétions/insertions et 31 présentant des microréarrangements du gène allant de la délétion d'un exon à celle du gène entier. La grande majorité des mutations ( $>90 \%$ ) étaient associées à une PS pure dans les familles. Cependant, quelques familles ont présenté des signes additionnels tels que des troubles cognitifs variables, une démence, un retard mental, une neuropathie périphérique ou un syndrome parkinsonien. Une grande proportion des mutations, plus particulièrement de type faux-sens (subsitutions d'acides aminés), a été identifiée chez des cas avec PS mais sans histoire familiale. L'analyse détaillée des familles a mis en évidence une pénétrance réduite et dépendante de l'âge. De plus, nous avons également identifié une mutation de novo associée à un mosaicisme somatique chez un des patients. Nos résultats sont en faveur de l'existence de facteurs modulateurs du phénotype pour expliquer pénétrance réduite et expressivité variable. Ces résultats ont des conséquences pour les indications de l'analyse moléculaire de SPG4, pour l'interprétation des résultats et pour le conseil génétique.

Mots-clés : paraplégie spastique héréditaire, SPG4/SPAST, MLPA.

\section{EP341 / 126. CARACTÉRISATION MOLÉCULAIRE DES POINTS DE CASSURE DU GÈNE CREBBP}

D. Simon (1), I. Burgelin (1), M. Stef (1), C. Rooryck (1, 2), D. Lacombe (2), I. Coupry (1), B. Arveiler $(1,2)$

(1) Laboratoire de Génétique Humaine, Pôle Génotypage Séquençage, Université Victor Segalen Bordeaux 2, France ; (2) Service de Génétique Médicale, CHU de Bordeaux, France

Introduction: Le syndrome de Rubinstein-Taybi (SRT) est une maladie rare, autosomique dominante causée par des mutations dans le gène CREBBP (CREB- binding protein) et plus rarement dans le gène EP300. Ce syndrome est connu pour être associé à des délétions du gène CREBBP dans 10 à $15 \%$ des cas. Matériel et méthodes : Par analyse en CGH-array et en PCR Quantitative Multiplex Fluorescente (QMF-PCR) nous avons montré que les réarrangements étaient présents chez $20 \%$ des patients, et que la taille des délétions observées était comprise entre $1245 \mathrm{pb}$ et $6,5 \mathrm{Mb}$. Notre objectif actuel est de mettre en évidence les mécanismes intervenant dans ces remaniements. La présence répétée de points de cassure dans certaines portions du gène conduit à penser que des séquences particulières, comme par exemple des duplications segmentales ou des séquences répétées sont présentes dans ces régions. Nous avons entrepris de séquencer 13 points de cassure intragéniques. Résultats : Actuellement, six délétions sont caractérisées, avec des tailles comprises entre $1245 \mathrm{pb}$ et $48327 \mathrm{pb}$. L'une d'entre elles est un réarrangement complexe avec une insertion d'un fragment de $435 \mathrm{pb}$ localisé à $3 \mathrm{~kb}$ du segment délété. Aucune séquence particulière n'a été identifiée à proximité du point de cassure. Chez un des patients, les séquences bordant le point de cassure sont des éléments AluSc et AluSx comportant 30 bases $100 \%$ identiques. Dans un autre cas, seules cinq bases sont identiques entre la séquence LINE L1ME2 et la séquence SINE MIR3, et enfin chez un autre patient, sept bases sont identiques avec à proximité une séquence AluSx d'un coté et de l'autre une séquence AluSg localisée à $250 \mathrm{pb}$. Dans les deux derniers cas, il n'y a pas de séquences homologues. Les sept autres réarrangements sont en cours de caractérisation. Conclusion et perspectives: Les résultats obtenus nous indiquent que les séquences Alu sont souvent impliquées dans les réarrangements intragéniques du gène CREBBP. Nous n'avons pas encore séquencé les points de cassure des duplications. En parallèle nous caractérisons également des points de cassure extragéniques car il serait intéressant de savoir si les mécanismes de réarrangements intragéniques et extragéniques sont différents.

Mots-clés : $C R E B B P$, points de cassure, séquences répétées.

EP342 / 128. MUTATION PRÉVALENTE AVEC EFFET FONDATEUR DANS LE XERODERMA PIGMENTOSUM DU GROUPE C EN AFRIQUE DU NORD : L'ESPOIR D'UN DIAGNOSTIC PRÉNATAL ET PRÉSYMPTOMATIQUE SIMPLIFIÉ

N. Soufir (1), C. Ged (2), A. Sarasin (3), A. Bourillon (1), C. Chemin (2), E. Bourrat (4), J. Armier (3), D. Pham (3), C. Blanchet-Bardon (4), K Khadir (5), M. Zghal (6), C. Robert (7), B. Bouadjar (8), A. Stary (3), A. Taieb (2), H. Benchiki (5), H. de Verneuil (2), B. Grandchamp (2)

(1) Laboratoire de Biochimie hormonale et génétique, Hôpital Bichat, APHP, Paris, France; (2) INSERM U876, Université V.-Segalen-Bordeaux-2 et CHU Pellegrin Bordeaux, France ; (3) Genomes and Cancers, FRE 2939 CNRS, Institut Gustave-Roussy, Villejuif, France ; (4) Service de Dermatologie, Hôpital Saint-Louis, APHP, Paris, France; (5) Service de Dermatologie, CHU Ibn Rochd, Casablanca, Maroc; (6) Service de Dermatologie, Hôpital Habib Thameur, TUNIS, Tunisie; (7) Service de Dermatologie, Institut Gustave-Roussy, Villejuif, France ; (8) CHU de Bab
Introduction : Le xeroderma pigmentosum (XP) est une génodermatose rare, transmise de façon autosomale récessive, causée par des mutations germinales de certains gènes de réparation de l'ADN (système NER). Les patients montrent une sensibilité extrême au soleil et présentent une fréquence de cancers cutanés environ 1000 fois supérieure à la population générale. Sept groupes de complémentation ont été rapportés (XP-A à XP-G), ainsi qu'un groupe variant (XPV ou POL H). Les groupes XP-C, XP-A, XPV sont les plus fréquents en Europe et en Afrique du Nord. La présence d'une mutation sur les deux allèles du gène de réparation concerné est responsable d'un phénomène d'hypermutagénèse qui conduit au développement anormalement précoce de cancers cutanés multiples. Patients et Méthodes : Nous avons étudié prospectivement 38 patients présentant une symptomatologie typique du XP, originaires en majorité d'Afrique du Nord: 18 patients suivis à Casablanca, 10 patients suivis à Alger et 10 patients suivis en France dont 6 originaires du Maghreb. Trois quarts des patients étaient issus de parents consanguins, aucun ne présentait d'atteinte neurologique. De plus, nous avons étudié rétrospectivement I'ADN provenant de lignées fibroblastiques de 15 patients XPC (10 originaires d'Afrique du Nord, 5 d'Europe) authentifiés préalablement par test de complémentation. Les ADN ont été préparés à partir de prélèvements sanguins ou cutanés, après obtention d'un consentement éclairé. Les mutations du gène $X P C$ ont été identifiées par séquençage automatique (région codante et jonctions intron-exon). Les haplotypes ont été reconstruits à l'aide de polymorphismes fréquents du gène XPC et de 2 microsatellites intragéniques. La mutation prévalente du gène XPA a été analysée par RFLP et/ou par séquençage de l'exon 6. Résultats et discussion : La mutation prévalente du gène XPA (p.R228X) est présente à l'état homozygote chez 5 patients $(13 \%)$, tous d'origine consanguine. Une mutation fortement prévalente du gène XPC (p.V548A fsX572) est présente à l'état homozygote chez 29 patients $(76 \%)$ dont la majorité d'origine consanguine. Cinq autres mutations du gène XPC (dont 4 non publiées) ont été identifiées chez 3 patients : 2 sont hétérozygotes composites et le $3^{\text {e}}$, homozygote, est issu de parents consanguins. Un haplotype rare est commun chez 18 patients porteurs de la mutation prévalente, originaires d'Afrique du Nord, ce qui suggère fortement un effet fondateur dans le bassin méditerranéen. Ces données ont une influence directe sur la stratégie à employer pour l'analyse moléculaire des patients XP, en tenant compte du degré élevé de consanguinité. L'étude des lignées XPC retrouve la mutation prévalente p.V548A fsX572 à l'état homozygote dans 10 cas ( 9 patients originaires d'Afrique du Nord et un d'Espagne). De plus, 5 autres mutations du gène XPC ont été identifiées, l'une chez un patient d'Afrique du Nord à l'état homozygote, les 4 autres chez des patients européens, à l'état hétérozygote composite. Conclusion: La forme XP-C, la plus commune en Europe et en Afrique du Nord, est une maladie invalidante, caractérisée par une lourde morbidité et une mortalité précoce. La fréquence très élevée de la même mutation chez les malades d'Afrique du Nord va considérablement simplifier le diagnostic moléculaire du XP dans le Maghreb. Ceci aura 2 conséquences: d'une part, faciliter le diagnostic prénatal de la maladie dans les familles qui le souhaitent, et d'autre part permettre la réalisation du diagnostic présymptomatique dès la naissance afin de mettre en place une photoprotection adaptée.

Mots-clés : genodermatose, effet fondateur, dignostic moléculaire.

EP343 / 134. RÔLE FONCTIONNEL DE L'ISOFORME LYMPHOCYTAIRE DE LA DYSTROPHINE TOUJOURS EN QUESTION : NOUVEAUX ÉLÉMENTS DÉDUITS DE L'ANALYSE D'UN SUJET BECKER PRÉSENTANT UN ALLÈLE COMPLEXE DU GENE DMD

P. Khau-Van-Kien, D. Thorel, C. Saquet, D. Méchin, S. Tuffery-Giraud, M. Claustres

Laboratoire de Génétique Moléculaire, CHU de Montpellier/INSERM U827

Introduction : La régulation transcriptionnelle du gène dystrophine, le plus grand gène humain est un phénomène complexe et peu connu, qui permet de générer selon les tissus de nombreuses isoformes de la protéine. Parmi celles-ci, l'existence et le rôle d'une isoforme lymphocytaire (Dp4271), spécifiquement transcrite à partir d'un promoteur (juxtaposé à l'exon 1 non codant) situé à plus de 4,5 Mégabases de l'exon 2 est débattue dans la littérature. Nous rapportons ici les données issues de l'analyse d'un cas index présentant une dystrophinopathie. Sujets et Méthodes - Sujet (cas index) étudié exhaustivement en génétique moléculaire: recherche de grands réarrangements (MLPA), étude des transcrits musculaires (RTPCR/PTT) et séquençage de la totalité des 79 exons et des 8 promoteurs - Etude de ségrégation familiale des variations identifiées - Etude épidémiologique des variations identifiées à partir de 164 chromosomes issus de la population générale et de 109 témoins masculins apparentés sains 
sélectionnés dans des familles DMD antérieurement caractérisées - Etudes in silico : conservation inter et intra espèces, modélisation 3D de la mutation c.170T>C (p.Leu57Pro). Résultats - Seules deux variations potentiellement délétères et non décrites sont identifiées chez le cas-index : 1 transition $\mathrm{G}>\mathrm{A}$ en +1 du site donneur d'épissage de l'exon 1 de l'isoforme lymphocytaire : IVS1+1G $>$ A (Dp4271) ; $2-$ c.170T $>$ C ou p.Leu57Pro (retrouvée sur les transcrits DMD sans anomalie d'épissage) dans l'exon 3 du gène $D M D$. - La ségrégation de ces deux variants dans la famille du cas index, montre que ceux-ci coségrègent en cis avec le statut des conductrices obligatoires. - La mutation c.170T $>\mathrm{C}$ (p.Leu57Pro) n'est pas retrouvée sur les 273 chromosomes témoins testés par contre, la deuxième variation : IVS+1G $>A$ (Dp4271) est identifiée sur l'ADN du frère sain d'un cas index Duchenne présentant une duplication de l'exon 2 du gène DMD. Cette variation est également retrouvée chez la mère conductrice asymptomatique (notamment pas d'élévation des CPK). - Les études complémentaires, conservation inter et intra espèces, prédictions in silico et modélisation 3D de la variation c.170T $>C$ (p.Leu57Pro) renforcent fortement son caractère délétère supposé (faux-sens situé au niveau du domaine de liaison à l'actine). Discussion/ Conclusion :La présence d'une variation altérant le site invariant GT d'épissage chez: 1 - un sujet témoin indemne de la maladie, 2 - en trans d'une mutation délétère chez sa mère conductrice obligatoire mais asymptomatique, 3 - en cis d'une autre mutation (p.Leu57Pro) dont la causalité est fortement suggérée ; indique que celle-ci correspond vraisemblablement à un polymorphisme rare, sans retentissement fonctionnel remarquable. Notre observation confirme certaines données de la littérature qui suggèrent un caractère artéfactuel et putatif de l'isoforme lymphocytaire Dp427l du gène dystrophine.

Mots-clés : dystrophine, isoforme lymphocytaire (Dp4271), myopathies de Duchenne et de Becker.

P344 / 135. LARGES DÉLÉTIONS DU GÈNE SHOX ET DE LA RÉGION PAR1 CHEZ UN PATIENT PRÉSENTANT UNE PETITE TAILLE ASSOCIÉE À UNE ÉPILEPSIE ET RETARD MENTAL

L. Mutesa, J.F. Vanbellinghen, K. Segers, M. Jamar, A.C. Hellin, G. Pierquin, V. Bours

Centre de Génétique Humaine, CHU de Liège Belgique

L'haploinsuffisance du gène SHOX (short-stature homeobox gene on the $\mathrm{X}$ chromosome) est habituellement associée à une petite taille idiopathique mais aussi à des anomalies radiographiques diverses. Cependant, des délétions au niveau de ce gène (Xp22.32-Yp11.3) et de la région PAR1 (pseudoautosomal region 1) voisine ont été mises en évidence dans la majorité des cas de dyschondrostéose Leri-Weill mais également dans une proportion significative des patients présentant une petite taille. Nous rapportons ici le cas inhabituel d'un patient présentant une petite taille associée à une épilepsie et retard mental idiopathiques, chez qui des délétions totales du gène $S H O X$ et de la région PARI ont été mises en évidence par les techniques de FISH (fluorescent in situ hybridization) et de MLPA (Multiplex Ligation-dependent Probe Amplification).

Mots-clés : gène SHOX, région PAR1, délétions.

P345 / 139. DU SYNDROME DE STÜVE-WIEDEMANN (SWS) AU SYNDROME DE CRISPONI

N. Dagoneau (1), S. Bellais (1), B. Leheup (2), P. Blanchet (3), P. Sarda (3), L.I. Al-Gazali (4), M. Di Rocco (5), C. Le Goff (1), A. Munnich (1), V. Cormier-Daire (1) et Le groupe international collaboratif sur le Syndrome de Stüve-Wiedemann

(1) Département de Génétique et INSERM U781, Hôpital Necker-EnfantsMalades, Paris, France ; (2) Département de Génétique, Hôpital Universitaire, Nancy, France; (3) Département de Génétique, Hôpital Arnaudde-Villeneuve, Montpellier, France; (4) Département de Pédiatie, Université des Émirats Arabes Unis, Al Ain, Émirats Arabes Unis ; (5) Seconde unité de Pédiatrie, Institut G.-Gaslini, Gênes, Italie

Le syndrome de Stüve-Wiedemann (SWS) est un syndrome autosomique récessif sévère caractérisé par une incurvation des os longs, avec un épaississement de la corticale interne, des anomalies métaphysaires, une camptodactylie, des difficultés alimentaires, un trismus, une détresse respiratoire et des épisodes d'hyperthermie responsables du décès dans les premiers mois de vie. Grâce aux prélèvements de 19 familles, nous avons localisé le gène responsable de la maladie en $5 \mathrm{p} 13$ puis identifié des mutations dans le gène LIFR (Leukemia Inhibitory Factor Receptor). Nous avons pu montrer dans les fibroblastes de patients SWS que la perte de fonction du LIFR entraîne une perturbation de la voie de signalisation JAK/STAT. Depuis, nous avons étudié 26 nouvelles familles SWS, et identifié 18 mutations dont 11 nouvelles dans 17 familles. Dans les 9 autres familles, une étude de liaison réalisée au sein de 4 familles consanguines nous a permis d'exclure le locus LIFR (5p13). De plus, pour 4 d'entre elles, l'étude de l'activation de la voie JAK/STAT en présence du LIF montre une activation normale. Le syndrome de Crisponi est proche du SWS caractérisé par les mêmes manifestations de dysautonomie mais distinct par l'absence de manifestation osseuse. Nous avons exclu une mutation LIFR dans ce syndrome pour trois familles comprenant 4 enfants atteints. Puis, nous avons considéré CRLF1 (Cytokine Receptor-Like Factor 1) impliqué dans le CISS (Cold-Induced Sweating Syndrome), comme gène candidat. Par séquençage direct, nous avons identifié dans $C R L F 1$ une mutation faux sens (c.226T $>\mathrm{G}$, p.W76G) et quatre mutations tronquantes (c.242G $>\mathrm{A}$, c. $527+5 \mathrm{G}>\mathrm{A}$, c.676dupA, c.707dupC) dans quatre familles à ce jour. L'ensemble de ces résultats nous permet de suggérer l'implication de la voie CNTFR dans les manifestations de dysautonomie alors que la voie LIFR aurait un rôle spécifique dans les manifestations osseuses.

Mots-clés : syndrome de Stüve-Wiedemann, syndrome de Crisponi, CRLF1.

\section{EP346 / 143. SYNDROME DE TREACHER-COLLINS : EXPLORA- TION MOLÉCULAIRE ET CARACTÉRISATION DE DEUX NOU- VELLES MUTATIONS}

C. Collet (1), C. Baumann-Morel (2), J. Callebert (1), A. David (3), J.M. Launay (1), M. Lenne (1), S. Marlin (4), D. Martin-Coignard (5), A. Verloes (2), J.L. Laplanche (1)

(1) Service de Biochimie et Biologie Moléculaire, Hôpital Lariboisière, Paris, France : (2) Service de Génétique Clinique, Hôpital Robert-Debré, Paris, France; (3) Unité de Génétique Clinique, Hôpital mère-enfants, Nantes, France ; (4) Service de Génétique Clinique, Hôpital Trousseau, Paris, France ; (5) Service de Génétique, CH du Mans, Le Mans

Le syndrome de Treacher-Collins (TCS) ou syndrome de FranceschettiKlein (OMIM 154500) est une affection congénitale responsable de malformations cranio-faciales de transmission autosomique dominante avec une pénétrance de $90 \%$ et une expressivité variable. Les principaux signes cliniques sont une hypoplasie des os malaires bilatérales symétriques, avec une obliquité anti-mongoloïde des fentes palpébrales, un colobome des paupières inférieures, une microtie et une fente palatine. Son incidence est estimée à $1 / 50000$ naissances. Le gène responsable de TCS est TCOF1, localisé en 5q32-33.1. De nombreuses mutations chez des patients atteints de TCS ont été rapportées dans les 28 exons du gène TCOFI. Il apparaît que cette pathologie résulte de mutations privées qui, dans leur majorité, introduisent un codon de terminaison prématuré dans la protéine TREACLE. Cette protéine localisée dans le noyau joue un rôle dans la production d'ARNr en s'associant avec le facteur de transcription UBF (upstream binding factor) lors de la transcription des gènes de l'ADN ribosomal. Il est supposé que les mutations dans le gène $T C O F 1$, pourraient entrainer une mauvaise localisation de la protéine tronquée et en conséquence, une perte des fonctions de TREACLE. Depuis la mise en place du diagnostic moléculaire au laboratoire en 2006, nous avons analysé l'ADN de 8 patients âgés de 4 ans à 26 ans ( 2 filles et 6 garçons), par dHPLC et séquençage direct bidirectionnel. Les 28 exons, incluant le 3'UTR, et la région promotrice ont été étudiés. Cinq mutations ont été détectées dans le gène TCOF1: c.305-1G>T, p.S617X, c.4135_4139delGAAAA ou c.4138_4142delAAGAA, p.P446NfsX17, p.P517AfsX9. Les mutations c.305-1G>T (site accepteur de l'exon 3) et p.P517Afs X9 (exon 11: NM 000356) sont nouvelles. Aucune mutation n'a été détectée chez trois patients, aussi la présence d'un polymorphisme dans les exons 21 et/ou 23 n'exclut pas une délétion hétérozygote de certains exons en amont, hypothèse actuellement en cours d'étude. Les cinq mutations que nous avons identifiées semblent toutes responsables de la production d'une protéine tronquée, résultats en accord avec les études précédemment publiées. L'intérêt de l'analyse moléculaire du gène TCOFI se justifie par l'importante variabilité intra familiale encore inexpliquée, la description d'un cas de mosaïcisme et les relations éventuelles avec le syndrome voisin de Goldenhar.

Mots-clés : syndrome de Treacher-Collins-Franceschetti-Klein, mutation, analyse moléculaire.

EP347 / 144. FORME PRÉCOCE D'HÉMOCHROMATOSE CHEZ DEUX PATIENTS PRÉSENTANT DE NOUVELLES MUTATIONS DE TFR2

A.M. Jouanolle (1), A. Mosser (1), V. David (2), S. Sauvion (3), M. Ropert (4), B. Turlin (5), O. Loréal (6), Y. Deugnier $(6,7,8)$, P. Brissot $(6,8)$ (1) Laboratoire de Génétique Moléculaire, IFR 140, CHU PontchaillouRennes; (2) UMR6061-IFR140, Université de Rennes I; (3) Service de Pédiatrie, CHU Jean-Verdier, Bondy ; (4) Laboratoire de Biochimie, CHU Pontchaillou, Rennes: (5) Département d'Anatomie et Cytopathologie, CHU Pontchaillou, Rennes; (6) Inserm U 522, IFR140, Université de 
Rennes 1 ; (7) CIC Inserm 0203, CHU Pontchaillou, Rennes; (8) Service des Maladies du Foie-CHU Pontchaillou, Rennes, Université

Introduction L'hémochromatose de type 3 due à des mutations du gène codant le récepteur 2 de la transferrine (TfR2) est une pathologie de surcharge en fer dont la présentation clinique et l'évolution sont le plus souvent similaires à celles de l'hémochromatose de type 1 relevant de l'homozygotie pour la mutation p.Cys282Tyr. La prévalence de cette entité n'étant pas connue, une étude portant sur des patients suivis dans notre centre ou adressés pour avis a été mise en place afin d'évaluer l'implication de TfR2 dans le développement d'une surcharge en fer. Patients et méthodes 31 patients non apparentés ont été inclus dans l'étude ; ils présentaient une élévation des paramètres sériques du bilan martial : coefficient de saturation de la transferrine supérieur à $60 \%$, taux de ferritine plasmatique compris entre 566 microgrammes/L et 7460 microgrammes $/ \mathrm{L}$. Tous avaient une surcharge hépatique en fer avérée, évaluée par coloration de Perl's sur ponction de biopsie ou par IRM. La surcharge en fer ne relevait ni des gènes $H F E$ et $S L C 40 A I$ ni des gènes HJV et HAMP. L'analyse du gène TfR 2 a été réalisée par séquençage des exons et des régions introniques flanquantes. Résultats Deux patients présentant des mutations de $T f R 2$ ont été identifiés. - Un jeune homme de 27 ans originaire de Bretagne qui consultait pour asthénie, arthralgies de la main droite et perturbation du bilan martial : élévation du fer sérique ( 48 micromoles/L), du coefficient de saturation de la transferrine $(64 \%)$ et de la ferritine plasmatique (1 015 microgrammes/L). L'analyse de la ponction de biopsie hépatique a révélé une fibrose de stade IV et une concentration en fer massive. Deux mutations du gène TfR 2 ont été mises en évidence, l'une dans l'exon 17 (p.Met705HisfsX87) non rapportée dans la littérature, l'autre dans l'exon 18 (p.Gly792Arg) déjà décrite. - Une fillette âgée de 11 ans née de parents consanguins originaires d'Afrique du Nord. Le bilan biologique réalisé pour asthénie a révélé des anomalies du bilan martial : fer sérique 50 micromoles $/ \mathrm{L}$, coefficient de saturation de la transferrine $98 \%$, ferritinémie 560 microgrammes/L. La concentration hépatique en fer évaluée par IRM atteignait 200 micromoles/g de foie sec $(\mathrm{N}>36)$. L'étude du gène $H F E$ avait mis en évidence une hétérozygotie pour la mutation p.His63Asp, aucune mutation n'avait été identifiée dans les gènes HJV et HAMP impliqués dans l'hémochromatose juvénile. L'étude du gène TfR2 a permis d'identifier une nouvelle mutation p.Arg678Pro retrouvée à l'état homozygote. Conclusion: Deux cas d'hémochromatose de type 3 relevants de mutations non décrites de $T f R 2$ ont été mis en évidence dans un groupe de 31 patients présentant une surcharge en fer inexpliquée. Le phénotype differe d'une hémochromatose de type 1 puisque les 2 patients présentent très jeunes des signes de surcharge hépatique en fer et qu'il n'existe pas de différence d'expression phénotypique entre les 2 sexes. Cette étude confirme l'existence d'une corrélation génotype-phénotype majeure dans l'hémochromatose de type 3. En pratique, le diagnostic d'hémochromatose relevant de $T f R 2$ devrait être évoqué devant une surcharge en fer survenant chez un sujet jeune n'impliquant ni $H J V$ ni HAMP.

Mots-clés : hémochromatose, HFE, TfR2.

EP348 / 146. LA LOCALISATION SUBCELLULAIRE DE L'HOMOLOGUE HUMAIN DE MUTS, HMSH4 EST RÉGULÉE D'UN PART, PAR UN EXPORT NUCLÉAIRE ACTIF DÉPENDANT DE CRM1 ET D'AUTRE PART, PAR SA DIMÉRISATION AVEC SON PARTENAIRE HMSH5

F. Lespinasse (1), F. Lahaye (1), S. Neyton (1), P. Staccini (2), V. PaquisFlucklinger (1), S. Santucci-Darmanin (1)

(I) UMR CNRS 6543, Faculté de Médecine, Nice; (2) Stic, Faculté de Médecine, Nice

Les protéines MSH4 et MSH5 appartiennent à la famille des homologues de la protéine bactérienne MutS. Il s'agit d'une famille de protéines hautement conservées au cours de l'évolution et impliquées dans différents aspects du métabolisme de l'ADN tels que la réparation des mésappariements de paires de bases et la recombinaison homologue. Plusieurs études indiquent que MSH4 et MSH5 fonctionnent sous forme d'un hétérodimère lors des étapes précoces et tardives du processus de recombinaison méiotique chez les mammiferes. Néanmoins, le rôle précis de ces protéines est encore mal compris et des travaux récents suggèrent que la protéine humaine MSH4 pourrait également exercée un fonction cytoplasmique. Nous montrons ici, que la protéine MSH4 est présente dans le cytoplasme et le noyau des cellules germinales et aussi de cellules somatiques transfectées, et nous démontrons, pour la première fois, que la localisation subcellulaire de MSH4 est régulée au moins en partie par un mécanisme d'export nucléaire actif. Nous observons en effet que la protéine MSH4 s'accumule dans le compartiment nucléaire après un traitement des cellules avec la leptomycine B, un inhibiteur spécifique de l'exportine CRM1. La
MSH4 nous a permis d'identifier deux séquences d'export (NES) fonctionnelles dans la région $\mathrm{C}$-terminale de cette protéine, ces deux séquences étant requises pour permettre un export nucléaire via la protéine CRM1. Nos résultats suggèrent que CRM1 est également impliquée dans l'export nucléaire de MSH5. Enfin, nous observons que la dimérisation des protéines MSH4 et MSH5 facilite la localisation nucléaire de ces protéines ce qui suggère que l'interaction entre MSH4 et MSH5 est potentiellement impliquée dans la régulation de leur trafic intracellulaire. La navette entre le cytoplasme et le noyau s'est avérée être un mécanisme important dans la régulation de protéines multifonctionnelles impliquées dans la réparation de l'ADN et le maintient de la stabilité génétique, telles que BRCA1, p53 et FANCA. Nos résultats suggèrent qu'une navette nucléocytoplasmique pourrait participer également à la régulation des fonctions des homologues de MutS, MSH4 et MSH5.

Mots-clés : gènes $M S H$, transport nucléocytoplasmique, recombinaison méiotique.

\section{T349 / 149. ANALYSE MOLÉCULAIRE DE 22 CAS ATTEINTS DE DYSTONIE DOPA-SENSIBLE SANS MUTATION PONC-} TUELLE DU GENE $\mathbf{G C H} 1$

F. Clot (1, 2, 3, 4), D. Grabli (1, 2, 3, 5), E. Roze (6), M. Vidailhet (1, 2, $3,5,7)$, A. Afenjar (4, 8), D. Doummar (8), A. Durr (1, 2, 3, 4), A. Brice $(1,2,3,4,5,7)$ et Réseau des Dystonies Françaises

(1) INSERM U679, Paris; (2) Université Pierre-et-Marie-Curie, UMR S679, Hôpital de la Pitié-Salpêtrière, Paris; (3) Institut Fédératif des Neurosciences (IFR70), Hôpital de la Pitié-Salpêtrière, Paris ; (4) AP-HP, Hôpital de la Salpêtrière, Département de Génétique, Cytogénétique et Embryologie, Paris ; (5) AP-HP, Hôpital de la Pitié-Salpêtrière, Fédération de Neurologie, Paris ; (6) AP-HP, Hôpital Saint-Antoine, Service de Neurologie, Paris ; (7) Université Pierre-et-Marie-Curie, École Médicale de la Pitié-Salpêtrière, Paris ; (8) AP-HP, Hôpital Armand-Trousseau, Service de Neuropédiatrie, Paris

La dystonie dopa-sensible (DRD pour Dopa-responsive dystonia) dans sa forme typique débute par des troubles de la marche pendant l'enfance dus à une dystonie des membres inférieurs pouvant s'étendre progressivement. Chez l'adolescent et le jeune adulte, un torticolis spasmodique ou une crampe de l'écrivain peut être la seule manifestation. La maladie se transmet sur le mode autosomique dominant ou récessif. Dans les formes à transmission autosomique dominante, de nombreuses mutations du gène de la GTP cyclohydrolase I ( $G C H I)$ ont été identifiées. Le gène code pour une enzyme limitante de la biosynthèse de la tétrahydrobioptérine (BH4) elle-même cofacteur important de la tyrosine hydroxylase responsable de la conversion de la tyrosine en L-dopa. La majorité des mutations décrites dans le gène sont des mutations ponctuelles, responsables de formes typiques de la maladie. Quelques grandes délétions exoniques ont été identifiées, mais leur fréquence reste probablement sous estimée car leur recherche n'est pas systématique. Récemment la technique MLPA (Multiplex Ligation-Dependent Probe Amplification), facilement réalisable et fiable, a été mise au point pour doser les exons du gène $G C H I$. De plus, deux autres gènes codant pour la sépiaptérine réductase (SPR) et pour la tyrosine hydroxylase $(\mathrm{TH})$ sont responsables de formes autosomiques récessives de $\mathrm{DRD}$, dont l'expression clinique est hétérogène. Afin de préciser la fréquence des mutations de ces gènes causant les DRD, nous avons recherché des délétions du gène GCHI par MLPA chez 22 patients non porteurs d'une mutation ponctuelle. Nous avons également analysé par séquençage les gènes $S P R$ et $T H$ chez les patients pour lesquels le gène $\mathrm{GCH} l$ était exclu. Nous avons identifié 6 patients porteurs de 5 délétions différentes à l'état hétérozygote dans le gène $\mathrm{GCH} I$ (délétion complète du gène, de l'exon 1, de l'exon 6 , des exons 2 à 6 et des exons 5 à 6 ). Trois des 6 patients étaient des cas $\propto$ sporadiques $»$ et 3 étaient des cas familiaux. L'âge de début moyen de la maladie était de 4,4 ans $(+/ 2,9)$. La dystonie avait débuté aux membres inférieurs chez tous les patients et un des patients présentait un retard mental. L'analyse par séquençage des autres gènes a permis d'identifier deux mutations dans le gène $S P R$ à l'état homozygote (c.596-2A > G et p.R150G) chez deux patients. L'un des patients présentait un retard mental et une dystonie qui a débuté au niveau des yeux et du tronc à l'âge de 6 ans. Le second patient présentait depuis la naissance des crises oculogyres et après quelques mois un syndrome parkinsonien. Enfin, nous avons détecté deux patients porteurs de mutations hétérozygotes composites dans le gène TH (p.F375L/S647G et p.R319P/G414R). Ces patients présentaient un tableau clinique à début précoce caractérisé par un retard moteur, un syndrome akinéto-rigide et une dystonie généralisée. Les 12 patients restants ( 9 cas isolés et 3 cas familiaux) pour lesquels aucune mutation n'a été identifiée dans les gènes étudiés, avaient un âge de début de la maladie de 6,7 ans $(+1-6,6)$. Trois des patients avaient une dystonie ayant débuté à un membre supérieur et à l'exception d'un patient 
qui présentait un discret retard mental, aucune caractéristique clinique particulière n'a été observée. Dans notre cohorte de patients DRD, non porteurs d'une mutation ponctuelle du gène $G C H 1$, l'utilisation de la MLPA a permis d'améliorer de $27 \%$ l'identification des mutations dans le gène $G C H 1$. D'autre part, le séquençage des gènes $S P R$ et $T H$ a permis d'identifier 4 patients supplémentaires porteurs de mutations. Ainsi sur les 22 patients DRD étudiés sans mutation ponctuelle du gène GCH1, $46 \%$ ont une origine génétique identifiée.

Mots-clés : dystonie dopa-sensible, MLPA-GCH1, séquençage TH-SPR.

\section{IP350 / 152. CDKL.5 ET ENCÉPHALOPATHIES ÉPILEPTIQUES} PRÉCOCES CHEZ LES FILLES : UN GÈNE À NE PAS NEGLIGER L. Lambert (1), A. Saunier (1), F. Verneau (1), F. Giuliano (2), B. Doray (3), A. Goldenberg (4), M.A. Nguyen Morel (5), P. Jonveaux (1), C. Philippe (1)

1) Laboratoire de Génétique, CHRU Brabois, rue du morvan, Vandæuvrelès-Nancy ; (2) Unité de Génétique Médicale, Hôpital de l'Archet II, CHU Nice; (3) Service de Génétique Médicale, Hôpital Hautepierre, Strasbourg ; (4) Unité de Génétique Clinique, Hôpital C.-Nicolle, Rouen ; (5) Département de Pédiatrie, Hôpital A.-Michallon, Grenoble

Les mutations dans le gène $C D K L 5 / S T K 9$ localisé en Xp22 sont responsables d'une encéphalopathie épileptique dominante liée à l'X. CDKL5 a été impliqué dans des spasmes infantiles syndromiques (syndrome de West) ainsi que chez des jeunes filles présentant un phénotype recoupant celui du syndrome de Rett (variant Hanefeld). L'immense majorité des allèles délétères de $C D K L 5$ entraîne l'apparition de convulsions précoces (avant 2 mois) de type spasme infantile. Jusqu'à présent, des mutations ponctuelles (faux-sens, décalages du cadre de lecture, anomalies de l'épissage) ont été principalement décrites chez des filles bien que peu de garçons avec épilepsie précoce aient été explorés à ce jour. Suite à l'implication du gène $C D K L 5$ dans des encéphalopathies épileptiques précoces, nous avons mis en place le criblage de ce gène en 2005 chez les patientes présentant un syndrome de Rett avec épilepise de caractère inaugural sans mutation délétère dans le gène $M E C P 2$. Par la suite, nous avons élargi notre recrutement aux encéphalopathies sévères et précoces ( $<6$ mois) ainsi qu'aux spasmes infantiles principalement chez la fille mais également chez le garçon. Au préalable, nous avions éliminé chez les sujets de sexe masculin les mutations récurrentes touchant les deux expansions de polyalanines dans le gène $A R X$. Nous avons mis en place un criblage du gène $C D K L 5$ par séquençage systématique des 23 exons ( $\mathrm{y}$ compris les exons 1, 1A et 1B non codants) ainsi qu' une recherche de grands réarrangements (RGT) par QMPSF (une PCR multiplexe pour l'analyse des exons $2,3,8,11,12,14,20$ et 21). Nous avons étudié une cohorte de 112 patients (22 garçons et 90 filles), dans la majorité des cas les deux gènes (MECP2 et $C D K L 5)$ ont été analysés. Outre des polymorphismes connus (publiés dans la littérature ou répertoriés dans la base de données italienne http://www.biobank.unisi.it/Elencorett.asp) nous présentons des nouveaux variants polymorphes ou délétères du gène $C D K L 5$. Toutes ces variations de séquences ont été mises en évidence par séquençage. Au total nous avons détecté un allèle délétère à l'état hétérozygote chez 7 filles sur 90 testées $(7,8 \%)$. Ces 7 patientes présentent toutes sans exception une épilepsie sévère très précoce (entre 2 semaines et 8 mois) et un profil d'inactivation de l'X aléatoire. L'analyse du gène $C D K L 5$ par QMPSF n'a révélé, à ce jour, aucun RGT. De la même façon, aucune mutation dans le gène $C D K L 5$ n'a été retrouvée chez des garçons avec encéphalopathie épileptique sévère précoce. Nous décrivons sept nouvelles mutations dans $C D K L 5: 5$ mutations faux-sens (p.Ala40Val chez deux patientes, p.Asp153Gly, p.Arg178Pro, p.Leu227Arg), une mutation affectant le site donneur d'épissage de l'intron 7 (c. $463+1 \mathrm{G}>\mathrm{A})$, une duplication d'un nucléotide entraînant un décalage du cadre de lecture (c.1311dupC). Toutes ces mutations sont des anomalies de novo. Les mutations faux-sens touchent des aa localisés dans le domaine serine-thréonine kinase (aa 13 à 297), elles sont sans effet sur l'épissage (tests in vitro sur ARN totaux extraits de sang prélevé sur tubes PAXgene). Notre étude démontre clairement qu'il est important de cribler le gène CDKL 5 chez les filles présentant une encéphalopathie épileptique sévère précoce avec ou sans signes cliniques évocateurs d'un syndrome de Rett, ce gène est sans doute beaucoup plus rarement impliqué dans l'étiologie moléculaire des épilepsies précoces chez les garçons. Nous n'avons pas détecté de grands réarrangements dans $C D K L 5$ par QMPSF, ce qui tend à démontrer que ce type de mutation n'est pas fréquent. Cependant nous n'avons analysé que 8 des 23 exons de CDKLS sur un panel de 90 filles et il sera nécessaire de cribler une plus large cohorte de patientes pour l'ensemble des exons afin d'évaluer la part des RGT dans les allèles délétères responsables de cette encéphalopathie épileptique dominante liée à l'X.

Mots-clés: CDKLS, éncéphalopathie épileptique précoce, spectre mutationnel.
- 351 / 153, QUANTIFICATION RELATIVE DE LA TRANSCRIP. TION DU GENE MECP2 CHEZ DES PATIENTES PRÉSENTANT UN SYNDROME DE RETT

L. Lambert, A. Saunier, C. Nemos, S. Yilmaz, P. Jonveaux, C. Philippe Laboratoire de Génétique, Hôpitaux de Brabois, CHRU, Vandouvre-lès-Nancy

Environ $10 \%$ des formes typiques de syndrome de Rett restent inexpliquées au terme d'une stratégie de diagnostic moléculaire comprenant 1) un séquençage complet du gène $M E C P 2$ (et de $C D K L 5$ pour la forme variante avec épilepsie sévère précoce) et 2) la recherche de grand réarrangement génomique touchant $M E C P 2$ par une méthode quantitative. Des altérations affectant le taux de transcrits du gène $M E C P 2$ pourraient être responsables d'une proportion des cas de Rett typiques sans mutation délétère touchant la séquence codante de $M E C P 2$. L'appréciation du niveau de transcription du gène $M E C P 2$ chez ces patientes est une approche indirecte pour repérer des altérations touchant des éléments régulateurs de la transcription de ce gène (en cis : promoteur, enhancer, silencer ou en trans : facteur de transcription) ou des séquences introniques importantes pour un épissage correct de $M E C P 2$. Nous avons donc choisi de développer une nouvelle approche diagnostique, permettant d'évaluer le niveau de trancription du gène $M E C P 2$ par quantification relative à l'aide du SYBR Green par la méthode des delta delta $\mathrm{Ct}$ en utilisant deux endogènes différents ( $\beta$-actine et ESD). Nous avons tout d'abord testé l'efficacité et la spécificité de notre méthode à l'aide de témoins sains qui montrent une faible variabilité interindividuelle du niveau d'expression des 2 transcrits MECP2_e1 et MECP2_e2 lorsque les ARN sont extraits de sang frais prélevé sur tubes PAXgene. À l'inverse, les essais de RT-qPCR sur ARN extraits de lymphoblastes de temoins sains ou de patientes montrent une forte variabilité interindividuelle. Après cette étape de validation technique, nous avons appliqué ce protocole de RT-qPCR pour rechercher, chez des patientes atteintes d'un syndrome de Rett typique et non porteuses d'une mutation délétère dans $M E C P 2$, une variation du niveau de transcription de MECP2_el et/ou MECP2_e2. Nous avons appliqué ce protocole de quantification relative sur des ARN totaux extraits du sang frais pour une série de 8 patientes Rett typiques. Nous avons détecté une diminution de 50 à $80 \%$ du taux des transcrits MECP2_el et MECP2_e2 dans 3 cas sur 8 . Pour ces trois patientes montrant une haploinsuffisance au niveau transcriptionnel, le profil d'inactivation de l' $\mathrm{X}$ au locus HUMARA est aléatoire. Des analyses complémentaires (Western blot, étude des séquences régulatrices de $M E C P 2$ ) sont en cours pour d'une part confirmer les résultats de RTqPCR au niveau protéique mais également pour rechercher une cause à cette diminution du taux de transcription, élément essentiel pour confirmer au niveau moléculaire le diagnostic clinique de syndrome de Rett chez ces trois patientes.

Mots-clés : syndrome de Rett, $M E C P 2, \mathrm{RT}$ qPCR.

IP352 / 155. EXPRESSION ET MÉTHYLATION DU GÈNE DU TRANSPORTEUR DE LA SÉROTONINE (SLC6A4) DANS DES LIGNÉES LYMPHOBLASTOÏDES DE PATIENTS AUTISTES

R. Tabagh $(1,2)$, S. Védrine (2), R.-A. Thépault (1), P. Vourc'h $(1,2), C$. R. Andres $(1,2)$

(1) INSERM 619; (2) Faculté de Médecine, 10, Bd Tonnellé, 37032 Tours Cedex, Université François-Rabelais de Tours, CHRU de Tours

Le gène $S L C 6 A 4$ (17q12), qui code le transporteur de la sérotonine (5-HTT), est un gène candidat dans l'autisme. En effet une augmentation du taux de sérotonine sanguine a été observée chez plus de $30 \%$ des patients autistes. Des inhibiteurs de la recapture de la sérotonine agissant sur 5-HTT sont efficaces pour traiter l'anxiété et l'agressivité chez les patients autistes. Nous avons étudié quatre polymorphismes du gène SLC6A4 (SNP A/G et 5 HTTLPR dans le promoteur, VNTR dans l'intron 2, SNP dans l'UTR3') dans des populations françaises de patients autistes $(n=103)$, de patients avec déficience mentale (DM) non syndromique $(\mathrm{n}=100)$ et d'individus contrôles $(n=164)$. Nous n'avons pas observé d'association entre la DM et les polymorphismes testés. Mais nous avons mis en évidence une association significative $(\& \# 61539 ; 2=13.38, \mathrm{p}=0.00025)$ entre l'autisme et l'allèle court du polymorphisme 5 HTTLPR situé dans le promoteur. (Odds ratio de 1,$93 ; \mathrm{CI} 95 \%: 1,35-2,75$ ). Nous étudions actuellement les niveaux d'expression et la méthylation de la région promotrice du gène SLC6A4 dans des lignées lymphoblastoïdes d'autistes portant différents génotypes de 5HTTLPR. Ces lignées sont cultivées avec ou sans BDNF (Brain derived neurotrophic factor), un facteur connu pour agir sur l'activité de 5-HTT. Nos résultats sont en faveur de l'implication du gène SLC6A4 dans l'autisme, et suggèrent plus précisément qu'une forme particulière du promoteur de ce gène pourrait être un facteur de susceptibilité à l'autisme. Remerciements : INSERM, Région Centre.

Mots-clés : gène du transporteur de la sérotonine, expression et méthylation, autisme. 
घP353 / 156. BASE DE DONNÉES MAROCAINE DES MUTATIONS HUMAINES - THE MOROCCAN HUMAN MUTATION DATABASE (MOHUMUDA)

I. Ratbi, A. Gati, A. Sefiani

Département de Génétique médicale, Institut National d'Hygiène, Rabat, Maroc

La population marocaine est hétérogène, composée notamment d'amazighs, d'arabes, de personnes d'origine negro africaines et d'une minorité de marocains de confession juive. Quelques millions de marocains vivent actuellement en dehors du pays en particulier suite aux immigrations de la deuxième moitié du siècle dernier. La population marocaine se caractérise par un taux de consanguinité élevé, près de $25 \%$ et une natalité qui reste importante malgré une forte diminution ces dernières années. Les maladies génétiques représentent un vrai problème de santé au Maroc et leur importance relative va croitre avec les progrès réalisés dans les domaines des maladies environnementales et infectieuses. Nous rapportons la construction d'une base de données marocaine des mutations humaines, initiée en 2007, et maintenue par l'équipe du Département de génétique médicale de l'Institut National d'Hygiène (Rabat, Maroc). Elle est hébergée par le site de l'Institut et librement accessible à l'adresse http://www.sante.gov.ma/Departements/INH/MoHuM uDa/index.htm. La base de données répertorie les mutations humaines et les polymorphismes des maladies génétiques identifiés chez la population marocaine natale et chez les patients d'origine marocaine vivant à l'étranger rapportées dans la littérature (Pubmed et autres ressources en ligne), lors des réunions scientifiques, ainsi que des mutations non publiées, directement soumises au comité scientifique de la base de données par courrier électronique. Les études de marqueurs microsattelites ne sont pas rapportées. Le but de cette base de données est de rendre accessible aux généticiens, chercheurs et professionnels de la santé, grâce à l'outil Internet, des informations régulièrement mises à jour sur les mutations géniques détectées dans la population marocaine, et d'encourager les projets de collaboration entre différentes équipes.

Mots-clés : base de données, mutations, Maroc.

IP354 / 157. ÉTUDE DE L'IMPLICATION DU GÈNE NF2 DANS LES SCHWANNOMES VESTIBULAIRES ET PROPOSITION D'UNE STRATÉGIE D'ÉTUDE

F. Petit (1), C.-M. Dhaenens (1), X.-N. Bonne (1), C. Piriou (1), C.-A. Maurage (2), C. Vincent (3), D. Allorge (1)

(1) Laboratoire de biologie moléculaire, Centre de biologie, CHRU de Lille; (2) Service d'anatomopathologie, Centre de biologie, CHRU de Lille; (3) Service d'Otologie et Otoneurologie, Hôpital Salengro, CHRU de Lille

Le schwannome vestibulaire est une tumeur bénigne, qui survient dans la grande majorité des cas de façon isolée et sporadique chez l'adulte ou le sujet âgé. Plus rarement, il survient dans un contexte de neurofibromatose de type 2 et se présente classiquement de façon bilatérale, en association avec d'autres tumeurs bénignes. Nous avons étudié l'implication du gène $N F 2$ dans le développement de schwannomes vestibulaires isolés sporadiques, ainsi que dans les formes atypiques de NF2 ne répondant pas aux critères conventionnellement reconnus. En effet, les critères diagnostiques cliniques utilisés pour la NF2 sont particulièrement non adaptés pour le diagnostic initial des patients se présentant sans schwannome vestibulaire bilatéral, notamment chez les patients à histoire familiale négative, et d'autant plus lorsqu'il s'agit d'une tumeur isolée. Chez ces patients, le pourcentage de mutations détectées dans l'ADN leucocytaire apparaît quasi-nul et seule l'analyse de l'ADN tumoral, quand elle est possible, semble apporter un bénéfice. Au total, les résultats de notre travail nous ont permis de redéfinir une stratégie d'étude du gène $N F 2$ en fonction de la présentation clinique.

Mots-clés : schwannome vestibulaire, neurofibromatose de type 2, NF2.

\section{IP355 / 170. MISE EN PLACE D'UN TEST DE FONCTIONNALITE IN VITRO POUR LES MUTATIONS FAUX-SENS DU GÈNE BMPR2 IMPLIQUÉ DANS L'HYPERTENSION ARTÉRIELLE PULMO-} NAIRE PRIMITIVE

F. Coulet (1), S. Martin (1), K. Montagne (1), F. Lebrin (1), B. Sztrymf (2), G. Simonneau (2), M. Humbert (2), F. Soubrier (1)

(1) Laboratoire d'Oncogénétique et d'Angiogénétique Moléculaire, Groupe hospitalier Pitié-Salpêtrière, Paris; (2) UPRES EA2705, Service de Pneumologie et Réanimation Respiratoire, Hôpital Antoine-Béclère, Clamart

L'hypertension artérielle pulmonaire (HTAP) (OMIM 178600) est une remodelage des artères pulmonaires de petit calibre menant à une insuffisance cardiaque droite et à une diminution marquée de la survie. La forme familiale de la maladie est liée à des mutations hétérozygotes sur le gène BMPR2 (Bone Morphogenetic receptor II) codant pour un récepteur de type II du BMP de la voie de signalisation de la superfamille du TGF-beta. La fixation du ligand BMP sur le récepteur BMPRII entrâne la formation d'un complexe avec le récepteur de type I qui est alors phosphorylé, et induit la phosphorylation des récepteurs de type Smads 1,5,8 qui se lient à Smad4 pour intégrer le noyau et activer ou inhiber des cibles transcriptionnelles de façon directe ou indirecte. Des mutations sur le gène BMPR2 sont retrouvées dans $60 \%$ des formes familiales et 20 à $30 \%$ des formes sporadiques d'HTAP. Une grande variété de mutations ponctuelles a été rapportée sur le gène BMPR2 (Lane, Nat Genet, 2000 ; Machado, Hum Mutat, 2006) : non-sens, avec décalage du cadre de lecture, ou faux-sens. L'interprétation clinique des mutations faux-sens est plus difficile que celle des mutations tronquantes. L'incidence sur la fonctionnalité des mutations faux-sens repose actuellement sur les arguments de conservation interespèces, d'identification de ces variations dans des populations de sujets sains, de caractères physico-chimiques des acides aminés concernés et de la description dans les bases de données de mutations identifiées. Pour une meilleure interprétation des mutations faux-sens, notre objectif est de mettre en place un test visant à caractériser in vitro la fonctionnalité de ces mutations. Le modèle cellulaire utilisé est un modèle de cellules endothéliales de souris « Mouse Embryon Endothelial Cells (MEEC)». Nous avons mis en place un test fonctionnel mesurant l'effet sur les cibles transcriptionnelles de la voie de signalisation BMP passant par la voie des Smads par mesure de luminescence après cotransfection d'un plasmide contenant un élément de réponse aux BMP en amont du gène rapporteur luciférase. Les transfections transitoires sont réalisées par cotransfection du plasmide rapporteur contenant l'élément de réponse au BMP, de 2 vecteurs (pcDNA6.2-GW/EmGFP-miRNA) permettant l'invalidation du gène $B M P R 2$ murin d'une part et de l'Activine A receptor de type II (ACVR2) murin d'autre part, ainsi que du vecteur d'expression de BMPR2 humain sauvage ou muté. Nous avons sélectionné 12 mutations d'intérêt réparties sur tout le gène $B M P R 2$, qui ont été introduites dans le vecteur par mutagénèse dirigée. Après transfection et traitement par le ligand BMP4 (50 $\mathrm{ng} / \mathrm{ml}$ ), nous mesurons la restauration de la réponse au BMP4 en présence de BMPR2 humain sauvage ou muté. Les mutations faux-sens que nous avons testées et qui sont situées dans le domaine kinase de la protéine (exons 5 à 11) ne permettent pas la restauration de la réponse au BMP, résultat permettant d'affirmer leur caractère délétère. En revanche, certaines mutations faux-sens ou tronquantes situées hors du domaine kinase, en c-terminal, ne compromettent pas la réponse au BMP suggérant la perte d'activité d'une autre voie de signalisation du récepteur, alternative aux Smads, dont l'invalidation peut prédisposer également à la maladie. Cette voie pourrait être celle des MAPkinases. En conclusion, d'une part, nous avons mis en place un test fonctionnel qui nous permettra de conclure sur l'aspect délétère ou non des mutations faux-sens situées dans le domaine kinase du gène BMPR2. D'autre part, nous avons démontré que l'effet de mutations tronquantes situées dans la queue cytoplasmique n'est pas médié par la voie des Smads mais par d'autres voies qu'il reste à explorer.

Mots-clés : BMPR2, HTAP, faux-sens.

\section{IP356 / 180. DIAGNOSTIC MOLÉCULAIRE DES ANOMALIES CONSTITUTIVES DES PROTÉINES DU SURFACTANT PULMO-} NAIRE : STRATÉGIE ET BILAN

D. Feldmann (1), L. Jonard (1), R. Abou Taam (2), M. Bahuau (3), M. Tredano (1), R. Couderc (1), A. Clément (4), J. de Blic (2), R. Epaud (4) (1)Service de Biochimie, AP-HP, Hôpital Armand-Trousseau, Paris ; (2) Service de Pneumologie Pédiatrique, AP-HP, Hôpital Necker-Enfants Malades, Paris; (3) Unité Fonctionnelle de Génétique, AP-HP, Hôpital Henri-Mondor, Créteil ; (4) Unité de Pneumologie Pédiatrique, AP-HP, Hôpital Armand-Trousseau, Paris ; Inserm, UMR-S U719, Paris ; Université Pierre-et-Marie-Curie, Paris

Des anomalies constitutives des protéines du surfactant pulmonaire ont été récemment décrites. Elles constituent un ensemble phénotypiquement hétérogène. Le déficit en protéine $B$ du surfactant (SP B), transmis sur le mode autosomique récessif (mutations du gène $S F T P B$ ), se caractérise par une détresse respiratoire gravissime du nouveau né à terme, peu ou pas sensible au traitement par le surfactant exogène et à la ventilation assistée. Les anomalies de la protéine SP C (gène SFTPC), de déterminisme autosomique dominant, ont été décrites chez des patients pédiatriques ou adultes atteints de pathologies interstitielles et/ou alvéolaires chroniques. Enfin, des mutations récessives du gène $A B C A 3$, codant pour une protéine membranaire de la famille ABC des transporteurs utilisant l'ATP, sont responsables de tableaux de détresse respiratoire relativement hétérogènes dans 
lesquels le métabolisme du surfactant est globalement affecté avec des déficits non spécifiques en protéine SP B et/ou des tableaux éventuellement évocateurs de protéinose alvéolaire. L'étude moléculaire des gènes SFTPB, $S F T P C$ et $A B C A 3$ constitue une aide diagnostique majeure pour cet ensemble hétérogène de pathologies pulmonaires et s'inscrit dans l'arbre décisionnel proposé. Cent-quarante-deux patients non apparentés atteints de détresse respiratoire néonatale inexpliquée $(n=42)$, de pathologie respiratoire définie par une hypoxie chronique et/ou des signes de bronchopathie et/ou insuffisance respiratoire chronique (toux productive, crépitants, tirage intercostal), en association à une radiographie du thorax anormale $(\mathrm{n}=100)$ ont été étudiés. La mutation prévalente de chacun des trois gènes (c.361C $>$ GAA ou 12 lins2 pour SFTPB, p.lle73Thr pour $S F T P C$ et p.Glu292Val pour $A B C A 3$ ) a été recherchée en première intention. Ensuite, le choix du séquençage de l'un des trois gènes a été orienté par la symptomatologie clinique et les examens complémentaires (étude du lavage broncho-alvéolaire, biopsie pulmonaire). -4 déficits en SP-B ont été identifiés: 3 patients étaient homozygotes pour la mutation c. $361 \mathrm{C}>\mathrm{GAA}$ et 1 patient était homozygote pour la mutation c. $672+2$ delTGAGG. - 13 pathologies liées à SP C ont été observés: la mutation p.Ile73Thr était présente chez 9 patients; 4 nouvelles mutations c.325-1G>A, c.424delC soit p.His142ThrfsX26, c.563T $>$ C soit p.Leu 188Pro, c.566G >A soit p.Cys189Tyr ont été identifiées. L'effet délétère de chacune de ces nouvelles mutations est probable. Aucune n'était présente dans 100 chromosomes contrôles. La mutation c.325-1G>A affecte le site accepteur d'épissage de l'exon 4 et un effet délétère dominant négatif de la délétion de l'exon 4 a déjà été démontré. La mutation c.424delC dans l'exon 4 induit des modifications d'acides aminés dans la partie terminale de l'exon 4 et dans l'exon 5 qui sont des régions cruciales pour la maturation du précurseur protéique proSP-C. Les nouvelles mutations faux sens observées sont situées sur des acides aminés conservés de la protéine, l'acide aminé 188 est un point chaud de mutation, alors que le résidu 189 est le siège d'un pont disulfure. Parmi les patients ayant une mutation du gène SFTPC, 9 avaient hérité la mutation d'un de leur parent et 4 présentaient probablement une néomutation. Une grande hétérogénéité phénotypique intrafamiliale a été observée. Un déficit en ABCA3 a été identifié chez un patient porteur du génotype Q215K/R288K. 3 patients portaient la mutation p.Glu292Val à l'état hétérozygote et la séquence complète de $A B C A 3$ est en cours d'étude chez ces patients. Les atteintes dues à des mutations dans $S F T P B, S F T P C$ ou $A B C A 3$ sont responsables de pathologies respiratoires suivant des mécanismes physiopathologiques qui commencent seulement à être élucidés. Cependant la complexité du métabolisme du surfactant, les familles de patients chez qui aucune anomalie moléculaire n'a encore été identifiée et les phénotypes intrafamiliaux variables suggèrent que de nombreux facteurs moléculaires restent encore à découvrir

Mots-clés : surfactant, SFTPB, SFTPC.

IP357 / 183. DIAGNOSTIC MOLÉCULAIRE DES ALPHA-DYSTROGLYCANOPATHIES NON LIÉES A FKRP

C. Bouchet (1), S. Vuillaumier-Barrot (1), M. Gonzales (2), F. EnchaRazavi (3), A. Yanagisawa (4, 5), S. Maugenre $(4,5)$, F. Leturq (6), S. Quijano-Roy $(4,7)$, P. Guicheney $(4,5)$, N. Seta (1), SOFFOET et réseau Français DMC (GIS-MR)

(1) AP-HP, Hôpital Bichat Claude Bernard, Biochimie Métabolique, Paris, France ; (2) AP-HP, Hôpital Armand-Trousseau, Génétique et Embryologie Médicale, Paris, France : (3) AP-HP, Hôpital Necker, Embryologie, Paris, France; (4) INSERM U582, Institut de Myologie, Groupe Hospitalier Pitié-Salpêtrière, Paris, France; (5) Université Pierre-et-MarieCurie Paris 6, UMR S582, IFRI4, Paris, France; (6) AP-HP, Hôpital Cochin, Biochimie et Génétique Moléculaire, Paris, France ; (7) AP-HP. Hôpital Raymond-Poincaré, Pédiatrie, Biochimie Métabolique, Paris, France

Introduction: l'alpha-dystroglycan (alpha-DG) est une des protéines majeures du complexe de la dystrophine. Des anomalies de O-glycosylation (O-mannosylation) de cette protéine sont à l'origine de certaines dystrophies musculaires congénitales (DMC) et de formes plus sévères d'apparition fætale, les lissencéphalies de type II (LIS II) ou syndrome de Walker Warburg (WWS). Matériel et Méthodes : Notre étude porte sur deux populations de patients : 58 familles présentant au moins un cas fœtal de LIS II inclus sur les critères suivants : hydrocéphalie, agyrie, leptoméninges épaissies, ectopie neurogliale, dysplasie cérébrale ; et 62 patients DMC de sévérités diverses mais présentant tous un défaut de glycosylation de l'alpha-DG sur muscle ou en western blot. Une famille comprend à la fois un cas LIS II et un cas DMC. Les gènes codant pour les glycosyltransférases potentiellement impliquées dans le processus de $\mathrm{O}$-mannosylation (POMT1, POMT2, POMGnT1, FCMD, LARGE) ont été séquencés sur
ADN génomique. Le gène LARGE a été dans un second temps étudiés au niveau du cDNA. Résultats: L'analyse de ces 119 familles d'alpha-dystroglycanopathies, nous a permis d'identifier le gène en cause pour 49 d'entre elles. 58 mutations différentes, dont 46 nouvelles ont été trouvées dans les 5 gènes étudiés. Le diagnostic moléculaire a pu être posé dans $52 \%$ des cas de LIS II : 15 familles POMTI, 9 POMGnTI, 5 POMT2, 1 LARGE, 0 FCMD. Pour POMTI, 21 mutations ont été identifiées, pour $P O M G n T 110$ mutations, pour POMT2 8 mutations et une mutation homozygote dans LARGE. Dans $31 \%$ des cas de DMC, le gène a été identifié, avec une prédominance de formes liées à POMT2: 10 POMT2, 3 POMGnTI, 3 FCMD, 2 POMTI, 1 LARGE. Huit familles POMT2 présentent la même mutation p.Tyr666Cys pour laquelle un effet fondateur a été trouvé. Cinq mutations ( 4 faux sens et un codon stop) ont été identifiées chez trois patients non apparentés dans le gène FCMD. Au total 21 mutations différentes ont été retrouvées pour les patients DMC. Discussion: Nous présentons ici le bilan de l'étude moléculaire des alpha-dystroglycanopathies non liées à FKRP regroupant à la fois les LIS II et les DMC. Cette étude montre une bonne efficacité de diagnostic moléculaire notamment pour les formes cliniques les plus typiques. La corrélation phénotype/génotype dans les LIS II nous permet désormais de cibler le diagnostic moléculaire en fonction de l'examen foetopathologique. Les cas les plus graves de LIS II, ectopie neurogliale sévère et atteintes multisystémiques semblent plus souvent liés à $P O M T 1$ et $P O M T 2$ tandis que les formes plus modérées, sans atteintes multisystémiques sont liées à POMGnT1. Les mutations POMT1 des LIS II se traduisent toutes par une protéine tronquée (codon stop, décalage du cadre de lecture ou mutation d'épissage) alors que les 2 patients atteints de DMC présentent au moins une mutation faux sens. La sévérité de la pathologie dépendrait donc non seulement du gène impliqué mais également du type de mutation. Chez les patients DMC, le gène POMT2 doit être étudié en priorité mais on ne peut exclure l'étude de POMTI. Trois patients atteints de DMC liée à FCMD ont été diagnostiqués, indiquant que ce gène doit aussi être étudié chez des patients non originaires du Japon. L'identification d'une mutation $L A R G E$ dans un cas de LIS II montre qu'il est important de séquencer ce gène non seulement dans des formes peu sévères de DMC comme initialement décrit mais également dans les formes plus sévères de type LIS II ou WWS.

Mots-clés : alpha-dystroglycan, dystrophie musculaire congénitale, Lissencéphalie type II

\section{P358 / 188. DOMINANT-NÉGATIVE GCMB MUTATIONS CAUSE HYPOPARATHYROIDISM}

M. Mannstadt (1), G. Bertrand (2), M. Muresan (3), G. Weryha (3), B. Leheup (4), SR. Pulusani (1), B. Grandchamp (2), H. Jüppner (1, 5), C. Silve $(2,6)$

(1) Endocrine Unit and (5) Pediatric Nephrology Unit, Massachusetts General Hospital and Harvard Medical School, Boston, MA, USA ; (2) AP-HP, Hôpital Bichat Claude Bernard, Service de Biochimie Hormonale et Génétique, Paris; (3) Service d'Endocrinologie, CHU de Nancy-Brabois, Nancy; (4) Service d'Endocrinologie Pédiatrique et Génétique Clinique, CHU de Nancy-Brabois, Nancy; (6) INSERM U773, Faculté de Médecine Xavier-Bichat, Paris

Hypoparathyroidism (HP) is characterized by low parathyroid hormone (PTH) levels, hypocalcemia and hyperphosphatemia. Heterozygous mutations in preproPTH or the calcium-sensing receptor (CaSR) cause some forms of autosomal dominant HP (AD-HP), while homozygous mutations in glial-cell missing $\mathrm{B}(G C M B)$ have been implicated in autosomal recessive HP. Importantly, heterozygous carriers of these mutations were healthy. The transcription factor GCM, first described in the fruit fly as a master regulator of glial cell development, belongs in mammals to a small family of key regulators of the development of parathyroid glands. We now report the identification of heterozygous GCMB mutations as the cause of autosomal dominant hypoparathyroidism in two unrelated families with several affected members ; in both families, mutations in the genes encoding PTH and the CaSR were excluded. Because of the dominant mode of inheritance, we therefore considered the possibility of dominant negative $G C M B$ mutations. Indeed, direct nucleotide sequence analysis of $G C M B$ led to the identification of two novel, heterozygous mutations in genomic DNA of the affected, but not in the unaffected individuals in each family. Both heterozygous $G C M B$ mutations are single nucleotide deletions within GCMB exon 5 (c1389delT and c1399delC) that introduce a frame-shift resulting in the truncation of the $\mathrm{C}$-terminal GCMB region, which contains the putative second transactivation domain. To further elucidate the mechanism through which these heterozygous GCMB mutations cause autosomal dominant hypoparathyroidism, we tested in HEK and DF-1 cells whether the mutant GCMB inhibits the actions of the wild-type transcription factor using a GCMB-activated luciferase reporter. These experiments 
demonstrated that both GCMB mutants impaired dose-dependently the transactivation capacity and thus the biological activity of wild-type GCMB ; when expressed alone, the mutants exhibited a reduced capacity to activate the reporter. These findings strongly indicate that the newly discovered GCMB mutants have dominant-negative properties. The identification of two similar heterozygous GCMB mutations and the dominant negative effect observed in vitro establish the molecular basis for this form of AD-HP and provide a plausible explanation for HP in both families. These results indicate that $G C M B$ is a novel gene candidate to be tested in autosomal dominant HP, in addition to PTH and CaSR.

Mots-clés : hypoparathyroidism, transcription factor, autosomal dominant.

\section{G359 / 190. X-LINKED MENTAL RETARDATION - FIVE YEAR EXPERIENCE}

M. Nawara (1, 2), M. Jurek (1), K. Szcza\&\#322 ;uba (1), J. Bal (1), T. Mazurczak (1)

(1) Departement de Genetique Medicale, Institut de la Mere et de l'Enfant, Kasprzaka 17a, 01-211 Varsovie, Pologne; (2) Postgraduate School of Molecular Medicine, Pasteura 3, 02-093 Varsovie, Pologne

Mental retardation is the main reason of referral to genetic counselling unit in the Institute of Mother and Child. Despite its high incidence, about $50 \%$ of patients remain without the complete molecular and clinical diagnosis. Our laboratory is the only one in Poland offering full molecular analysis of Fragile X syndrome. Since 1992 we have collected in our database 2795 FraX negative patients. Out of this group we have selected patients for further investigations of X-linked mental ratardation (XLMR). Selected families (204) were divided into two groups: a) 94 pXL (potentially $\mathrm{X}$-linked) with at least two males affected in a family and one obligate female carrier ; b) $110 \mathrm{BP}$ (brother pairs) with at least two brothers affected. In these BP families 26 had three or more affected brothers. In the group pXL we also included 10 , and in the group of BP 3 families with less severely affected females. All probands were tested for mutations in the coding sequence of $A R X$ and $M E C P 2$. We identified five duplications in $A R X$, and one mutation and seven polymorphic variants in $M E C P 2$. All patients with severe/moderate mental retardation were also analysed for MECP2 duplications. The duplication was confirmed in three cases. In one patient we identified a deletion in SLC6A8 gene by using the same MLPA kit. Additionnaly, FRAXE analysis was previously performed for total number of 842 patients (most of them sporadic, few with cytogenetic fragile site). No FRAXE expansion was found. We also recontacted many families from the group $\mathrm{PXL}$ and collected samples for linkage analysis of 181 individuals from 18 families. For two large families a lod score $>2$ was calculated. For both probands known MRX (nonspecific XLMR) genes in the linkage intervals were analysed. In one family a partial deletion of ILIRAPLI was identified. For small families with lod score $<2$ the exclusion mapping and mutation analysis is in progress. In total, causative mutations were identified in ten families.

Mots-clés : retard mental, analyse de liaison, ARX.

\section{IP360 / 191. MUTATIONS DU GÈNE DE L'ANGIOGÉNINE DANS UNE POPULATION FRANCAISE DE PATIENTS ATTEINTS DE SCLÉROSE LATÉRALE AMYOTROPHIQUE SPORADIQUE}

A. Paubel $(1,2)$, P. Vourc'h $(1,2)$, J. Praline $(1,3)$, M. Amy (1), C. Cherpi-Antar (1, 2), W. Camu (4), P. Corcia $(1,3)$, C. Andres $(1,2)$, Groupe Français de Recherche de la SLA

(1) INSERM U619-CHU de Tours; (2) Service de Biochimie-CHU de Tours ; (3) Service de Neurologie, CHU de Tours ; (4) Service de Neurologie-CHU de Montpellier

La Sclérose Latérale Amyotrophique (SLA) est une affection neurodégénérative secondaire à une perte progressive des neurones moteurs du tronc cérébral, de la mœlle épinière et/ou du cortex cérébral, dont l'évolution est fatale en 2 à 5 ans. En France, elle touche environ 1000 personnes par an et les formes familiales représentent $10 \%$ des cas.

Plusieurs facteurs intervenant dans la néovascularisation ont été impliqués dans la physiopathologie de la SLA. Des haplotypes corrélés à une faible concentration sanguine de VEGF (Vascular Endothelial Growth Factor) ont été associés à un risque plus élevé de SLA. Des mutations du gène ANG (14q11.2) codant l'Angiogénine, un inducteur de la néovascularisation, ont récemment été identifiés dans des populations de SLA sporadique et familiale. Le gène ANG est composé d'un exon et code une protéine de 123 résidus d'acides aminés appartenant à la famille des ribonucléases A,dont le rôle physiologique exact reste méconnu. Nous avons séquencé la région codante du gène ANG chez 800 patients d'origine française atteints de SLA sporadique. Nous avons identifié trois mutations faux sens

différentes chez quatre patients (K17R, I46V $\times 2, \mathrm{R} 124 \mathrm{H})$. La mutation
R124H (G434A), affectant une arginine située dans la région C-terminale correspond à une nouvelle mutation du gène de l'Angiogénine dans la SLA. Des tests fonctionnels permettront de montrer si ces mutations entraînent une perte de fonction de l'Angiogénine. Nos résultats sur une grande population française de patients atteints de SLA sporadique confirment l'implication possible du gène de l'Angiogénine dans l'étiologie de cette maladie neurodégénérative pour laquelle il n'existe encore aucun traitement curatif.

Mots-clés: sclérose latérale amyotrophique, angiogénine, maladie neurodégénérative.

IP361 / 196. RECHERCHE DE MUTATIONS PONCTUELLES DANS LE GENE FMRI

N. Boutry-Kryza (1), G. Lesca (1), P. Edery (2), V. Des Portes (3), M.P. Cordier (4), J. Lespinasse (5), A. Calender (1)

(1) Laboratoire de Génétique Clinique et Moléculaire, Hôpital Edouard Herriot, Hospices Civils de Lyon; (2) Service de Cytogénétique Constitutionnelle, Centre de Biologie et de Pathologie Est, Hospices Civils de Lyon ; (3) Service de Neuropédiatrie, Hôpital Debrousse, Hospices Civils de Lyon: (4) Consultation de génétique, pavillon $K$, Hôpital ÉdouardHerriot, Hospices Civils de Lyon; (5) Service de Cytogénétique, hôpital de Chambéry

Le syndrome de l'X fragile est la première cause de retard mental lié au chromosome X. Sa fréquence est estimée à $1 / 4000$ hommes et $1 / 6000$ femmes. Sur le plan clinique, les patients présentent un retard mental sévère à modéré, une dysmorphie caractéristique mais inconstante et une macro-orchidie présente après la puberté. La confirmation du diagnostic au niveau moléculaire est très importante pour le conseil génétique. La maladie est causée, dans sa forme courante, par l'expansion d'une répétition de triplets CGG située dans la partie 5' non traduite du gène FMRI, associée à une méthylation anormale du locus et aboutissant à l'absence de production de la protéine FMRP. À côté de plusieurs délétions de grande taille, seules trois mutations ponctuelles ont été décrites jusqu'à présent. Elles comprennent une insertion/délétion de $2 \mathrm{pb}$, une délétion d'une $\mathrm{pb}$ ainsi que la substitution $1304 \mathrm{~N}$, située dans la région codant pour le domaine $\mathrm{KH} 2$ de la protéine. L'étude moléculaire du gène $F M R 1$ est indiquée chez la plupart des patients présentant un retard mental mais, compte tenu de la taille du gène, seule l'expansion de trinucléotides est recherchée dans le cadre du diagnostic de routine. L'objectif de notre étude était d'estimer la prévalence des mutations ponctuelles du gène $F M R I$ dans une cohorte de 50 patients présentant un retard mental non syndromique sans cause identifiée et sans expansion CGG. Les patients ont été recrutés dans le cadre d'une consultation de génétique médicale ou de neuropédiatrie et ont bénéficié d'un bilan étiologique étendu. Nous avons séquencé les 17 exons du gène FMRI, ainsi que les jonctions intron-exon chez ces patients et dans un groupe de 100 témoins. Nous avons mis en évidence 7 variations de séquence. Cinq d'entre-elles correspondent à des polymorphismes et sont retrouvées avec une fréquence similaire chez les témoins et les patients. Elles comprennent deux substitutions exoniques isosémantiques (c.18G > T et c.144G > A) et trois mutations introniques (c. $801+31 \mathrm{C}>\mathrm{T}$, c. $990+14 \mathrm{C}>\mathrm{T}$ et c. $1654+59 \_60$ dupTG). La substitution c. $1737+8 C>A$, située dans l'intron 16 est retrouvée chez un seul patient. L'étude de l'ARNm ne retrouve pas de conséquence au niveau de l'épissage. La substitution isosémantique c. $651 \mathrm{G}>\mathrm{A}$, située dans l'exon 8 est présente chez deux patients mais absente chez les témoins. Nous ne disposons pas d'ARNm pour étudier un éventuel effet sur l'épissage. Bien que nous n'ayons pas mis en évidence de mutation pathogène dans le groupe de patients étudiés, le gène $F M R I$, de par sa fonction, reste un bon candidat chez les patients porteurs de retard mental potentiellement lié à I'X. Le développement de tests protéiques et de techniques de biologie moléculaire à haut-débit permettrait de mieux cibler les patients candidats à de telles études et de réévaluer la participation de ces mutations dans les retards mentaux liés à l'X.

Mots-clés : X-fragile, mutation ponctuelle, séquençage.

D362 / 203. DÉLÉTION DU GÈNE DE LA PROGRANULINE : UNE NOUVELLE CAUSE DE DÉMENCE FRONTOTEMPORALE A. Rovelet (1), S. Legallic (1), F. Pasquier (2), I. Leber (3), T. Frebourg (1), D. Campion (1)

(1) Inserm U614, Faculté de Médecine et de Pharmacie, Institut Hospitalo-Universitaire de Recherche Biomédicale de Rouen; (2) Service de Neurologie, CHU de Lille; (3) Inserm U679, Hôpital de la Pitié-Salpêtrière, Paris

Ces dernières années, le développement de méthodes d'analyse des remaniements chromosomiques telles que la CGH-array a permis de mettre en 
exergue l'importance des variations du nombre de copies (CNV) en génétique humaine, notamment dans le développement de pathologies sporadiques, dues à des altérations de novo. Par la suite, il a également été mis en évidence un rôle des CNVs dans la transmission de pathologies mendéliennes, notamment dans le cas de duplications segmentaires de gènes observées dans la maladie d'Alzheimer et la maladie de Parkinson. L'étude des réarrangements génomiques dans les maladies neurodégénératives à transmission autosomique dominante permet non seulement d'identifier des altérations causales, mais également de mieux comprendre les mécanismes physiopathologiques de ces maladies. Les démences frontotemporales (DFT) sont les démences les plus répandues chez les individus de moins de 65 ans, et sont transmises de façon mendélienne dans la moitié des cas. Plusieurs gènes ont été impliqués dans ces formes autosomiques dominantes : le gène $V C P$ (Valosin-containing protein), situé en $9 \mathrm{p} 21$, le gène CHMP2B (Charged Multivesicular Protein 2B), situé en 3p13, le gène MAPT (Microtubule-associated Protein), situé en 17q21-22 et plus récemment le gène PGRN (Progranuline), également situé en 17q21-22, qui code pour un facteur de croissance. De nombreuses mutations ont été identifiées sur le gène PGRN, au niveau des régions exoniques ou introniques. Ces mutations de type non sens ou d'épissage ont pour conséquence commune d'aboutir à une perte d'expression de l'allèle muté. Dans ce cadre, nous avons émis l'hypothèse qu'une délétion du gène de la progranuline pourrait également être à l'origine de DFT. Afin de tester cette hypothèse, nous avons utilisé la technique de QMPSF (Quantitative Multiplex of Short Fragments), une méthode basée sur l'amplification simultanée de courts fragments génomiques dans des conditions quantitatives et permettant la détection d'anomalies quantitatives hétérozygotes. Nous avons analysé 60 patients recrutés par le CHU de Lille présentant une DFT, ainsi que 120 sujets contrôles. Dans une famille présentant une forme autosomique dominante de DFT, nous avons identifié une délétion impliquant l'ensemble des exons du gène de la progranuline. L'exploration par QMPSF des gènes adjacents a révélé que seul ce gène était délété, et par la suite les bornes de la délétion ont été déterminées par séquençage. Une délétion du gène de la progranuline est donc à l'origine d'une forme autosomique dominante de DFT. Ceci non seulement confirme le mécanisme d'haploinsuffisance de la progranuline dans la physiopathologie des DFT, mais est également une nouvelle preuve du rôle causal des CNVs dans les maladies neurodégénératives.

Mots-clés : Progranuline, CNV, QMPSF.

\section{DP363 / 204. SCREENING OF THE CHOROIDEREMIA GENE IN} CANINE PROGRESSIVE RETINAL ATROPHY

L. Robert (1), A. Sénéchal (1), A. Thomas (2), C. André (3), C. Hamel (1)

(1) INSERM U583, Institut des Neurosciences de Montpellier ; (2) Société Antagène, Limonest; (3) CNRS 6061, Université Rennes I

Introduction : Choroideremia is an X-linked, inherited retinal dystrophy involving the choroid, the retinal pigment epithelium and the photoreceptors, leading patients to complete blindness. $C H M$, the gene for choroideremia, located on chromosome Xq21.2, is ubiquitously expressed. The CHM product is known as the Rab escort protein (REP)-1, which prenylates small Rab proteins in the eye. In choroideremia, all CHM mutations currently identified lead to C-terminal truncated proteins or to the loss of the protein. The clinical features and some genes of canine progressive retinal atrophies (PRA) still remain poorly described in many breeds. In particular, it is not known whether choroideremia exists in dogs. We therefore searched for mutations in a panel of dogs with PRA. Finding mutations on this gene could allow to potentially using dogs as models of gene therapy in choroideremia. Matériel et Méthodes. Genomic DNAs of dog representatives from 72 breeds and 4 crossed animals were randomly amplified. The 15 exons and flanking sequence of $\mathrm{CHM}$ were PCR-amplified and subjected to direct sequencing. The resulting sequence was compared with the CHM mRNA deposited in Genbank (XM_538092). Résultats : We first carried out a literature search of all the $\mathrm{CHM}$ mutations currently identified in humans since 1987 . We found 89 nonsense, splice site or frameshift (deletion or insertion) mutations, 3 translocations and 40 exon skipping. These mutations are distributed along the gene with a majority situated in exons 5 and 6 . We then sequenced $C H M$ in 88 DNA samples from dogs. On the 5 exons sequenced so far, we detected three variants. Two of them, c. $314+64 A>G$ (intron 4) and c.1411-9A $>\mathrm{G}$ (intron 11), were found in 18 and $20 \%$ of the studied population, respectively. It is of note that the intron 11 variant is found in the consensus acceptor splice site of exon 12. The third variant, c. $1478 \mathrm{C}>\mathrm{A}$ was found in exon 12 and leads to Ser493Tyr. It was found in only one dog which is a male Gordon setter. Discussion: The finding of Ser493Tyr in one dog suggests that it could be a rare polymorphism or a mutation. Serine 493 is indeed conserved in mammals, exposed and predicted as a functional residue (ConSeq program). In addition, the tyrosine at this position is not tolerated and is predicted to affect the protein function with a score of 0.05 (SIFT program). The sequencing of the other $\mathrm{CHM}$ exons and investigations on Ser493Tyr are undergoing.

Mots-clés : choroideremia, progressive retinal atrophy, canis familiaris.

\section{-P364 / 217. LES DÉPLETIONS DE L'ADN MITOCHONDRIAL SONT UNE CAUSE MAJEURE DE DÉFICIT MULTIPLE DE LA CHAÎNE RESPIRATOIRE CHEZ L'ENFANT}

E. Sarzi (1), A. Bourdon (1), D. Chrétien (1), M. Zarhrate (2), J. Corcos (2), A. Slama (3), V. Cormier-Daire (1, 2), P. de Lonlay (4), A. Munnich $(1,2)$, Agnès Rötig (1)

(1) INSERM U781, Hôpital Necker-Enfants Malades, 149 rue de Sèvres, 75015 Paris : (2) Service de Génétique, Hôpital Necker-Enfants Malades, 149 rue de Sèvres, 75015 Paris ; (3) Laboratoire de Biochimie 1, Hôpital Bicêtre, Le Kremlin-Bicêtre; (4) Maladies du Métabolisme, Hôpital Necker-Enfants Malades, 149 rue de Sèvres, 75015 Paris

Les déplétions de l'ADN mitochondrial (ADNmt) consistent en une réduction importante du nombre de copies de l'ADN mitochondrial et conduisent à un déficit enzymatique de la phosphorylation oxydative. Elles sont dues à des mutations de gènes nucléaires impliqués dans le maintien de l'ADNmt. Les déplétions regroupent un ensemble cliniquement et génétiquement hétérogène de patients. Afin de déterminer l'incidence des déplétions de l'ADN mitochondrial dans les déficits multiples de la chaîne respiratoire, nous avons mis au point la quantification de l'ADNmt par PCR quantitative en temps réel. Cette quantification a été réalisée dans le foie et/ou le muscle de 300 patients présentant un déficit multiple de la chaîne respiratoire. Nous avons ainsi mis en évidence que 126 patients présentaient une diminution du nombre de copies de l'ADNmt (inférieure à $35 \%$ d'ADNmt résiduel). Une grande majorité de ces patients (55\%) présente une forme néonatale avec une atteinte hépatique sévère, un second groupe présente une encéphalopathie convulsivante et une hyperlactacidémie $(25 \%)$. Un troisième groupe comprend les syndromes d'Alpers (13\%) et enfin un dernier groupe de patients présente des atteintes cliniques plus hétérogènes telles qu'encéphalopathies, myopathies, tubulopathies, retards psychomoteurs...Des mutations ont été mises en évidence majoritairement dans les gènes DGUOK et $P O L G$ pour le groupe des atteintes hépatocérébrales et les syndromes d'Alpers respectivement. Cinq patients présentant une encéphalopathie et une tubulopathie ont des mutations dans un nouveau gène de déplétion $p 53 R 2$ et un seul patient atteint de myopathie présentait une mutation dans le gène $T K 2$. Aucune mutation n'a été retrouvée pour le groupe de patients avec encéphalopathie convulsivante et hyperlactacidémie ou cardiomyopathie. Cette étude a permis de montrer que les déplétions de l'ADNmt sont une cause majeure de déficit multiple de la chaîne respiratoire en période néonatale. Cependant dans $70 \%$ des cas, la mutation responsable n'a pu être identifiée. Publié dans The Journal of Pediatrics. 2007 May ;150(5) :531-4, 534.e1-6

Mots-clés : déplétions, DGUOK, POLG.

- 9365 / 220. UNE NOUVELLE MUTATION DANS LE GÈNE MITOCHONDRIAL CODANT POUR LE CYTOCHROME B (MTCYB) RESPONSABLE D'UNE DÉFAILLANCE POLYVISCÉRALE NÉONATALE

K. Fragaki (1), S. Bannwarth (1), G. Augé (1), F. Casagrande (2), J.-C Lambert (1), V. Paquis-Flucklinger (1)

(1) Service de Génétique Médicale, Centre de référence des pathologies mitochondriales, $\mathrm{CHU}$ de Nice ; (2) Service de Pédiatrie, CHU de Nice

Les déficits de la chaîne respiratoire sont extrêmement hétérogènes en termes de présentation clinique, de déficit biochimique et d'origine génétique. Néanmoins, les mutations identifiées dans le gène mitochondrial $M T C Y B$ sont presque toujours responsables d'un déficit en complexe III dans le muscle avec une présentation clinique de type intolérance à l'effort. Nous décrivons un enfant né à terme, eutrophique, qui a présenté une détresse respiratoire en salle de naissance. Les parents sont non consanguins et il n'existe aucun antécédent familial. L'examen retrouve une hypotonie avec une souffrance cérébrale diffuse et des convulsions infracliniques subintrantes focalisées à l'EEG. Le reste du bilan objective une acidose lactique majeure, une insuffisance hépatocellulaire et une myocardiopathie hypertrophique. L'enfant décède à $24 \mathrm{~h}$ de vie dans un tableau de défaillance polyviscérale. Le dosage des complexes de la chaîne respiratoire ne retrouve pas de déficit dans le muscle, mais un déficit isolé en complexe III est identifié à partir du prélèvement hépatique. Le séquençage du gène $M T C Y B$ a permis de retrouver une nouvelle transition $\mathrm{T} \rightarrow \mathrm{C}$ en position 15635 qui entraîne le remplacement d'un acide aminé hydrophile 
par une proline (S297P) dans un domaine transmembranaire, très conservé, de la protéine. Cette transition est retrouvée à un haut niveau d'hétéroplasmie dans tous les tissus étudiés (muscle, foie, myocarde, leucocytes et fibroblastes) ; ceci pouvant expliquer la gravité du tableau clinique présenté. L'étude des ascendants maternels, sur différents types cellulaires, devrait permettre de vérifier si l'hypothèse d'une origine sporadique peut être envisagée. De plus, la mutation m.15635 n'est pas présente dans la base de données MITOMAP (www.mitomap.org/) alors qu'elle est retrouvée 1 seule fois dans $\mathrm{mtDB}$ (www.genpat.uu.se/mtDB/), indiquant que cette transition n'est pas retrouvée fréquemment dans la population générale. Tous ces éléments sont en faveur du caractère pathogène de cette mutation qui devrait être confirmé par les tests fonctionnels en cours.

Mots-clés : mutation dans le gène $M T C Y B$, complexe III, déficit de la chaîne respiratoire.

\section{-P366 / 222. TRANSLECTURE DE CODONS STOP PRÉMATURES PAR LA GENTAMICINE : APPLICATION AU SYNDROME DE STÜVE-WIEDEMANN}

Samuel Bellais, Nathalie Dagoneau, Carine Le Goff, Arnold Munnich, Valérie Cormier-Daire

Département de Génétique Médicale et INSERM U 781, Hôpital NeckerEnfants Malades, Paris, France

Le syndrome de Stüve-Wiedemann (SWS) est une chondrodysplasie sévère caractérisée par une incurvation des os longs associée à des accès d'hyperthermie responsables du décès dans les premiers mois de vie. Aucun traitement n'est actuellement disponible pour ce syndrome rare transmis sur un mode autosomique récessif et en rapport avec des mutations dans le gène LIFR (Leukemia Inhibitory Factor Receptor). Nous avons pu montrer que la perte de fonction du récepteur au LIF entraîne une perturbation de la voie de signalisation JAK/STAT. L'identification de mutations non sens chez les patients nous permet d'envisager une approche pharmacologique basée sur la capacité des aminoglycosides à favoriser la translecture de codons stop prématurés. Nous disposons des fibroblastes de 6 patients SWS porteurs de mutations de type codon stop prématuré à l'état homozygote dans le gène LIFR et nous avons montré pour chacun une absence de phosphorylation de STAT3 en présence de LIF. Nous disposons également de fibroblastes contrôles et nous avons ainsi pu déterminer à l'aide de ce test fonctionnel, qu'après un traitement de $48 \mathrm{~h}$ par $200 \mathrm{ou} 500 \mathrm{mg} / \mathrm{l}$ de gentamicine le taux de restauration du signal pouvait atteindre environ $5 \%$ dans le cas d'un codon stop prématuré de type UGAc. L'efficacité de translecture varie en effet en fonction de l'environnement nucléotidique proche du codon stop impliqué. Nous avons également étudié la stabilité des ARNm LIFR de fibroblastes de patients traités ou non par $200 \mathrm{mg} / \mathrm{l}$ de gentamicine. Les analyses préliminaires de RT-PCR quantitative révèlent que la gentamicine augmente d'un facteur trois la stabilité des ARNm LIFR présentant un codon stop prématuré de type UGAc chez deux de nos patients. Nous venons d'obtenir la molécule PTC124 auprès de la firme PTC Therapeutics permettant de poursuivre les essais de translecture de codons stop prématurés sur nos fibroblastes de patients avec une molécule peu toxique et au potentiel supérieur à celui de la gentamicine dans l'optique de futurs essais cliniques.

Mots-clés : syndrome de Stuve-Wiedemann, translecture de codons stop prématurés, aminoglycosides.

\section{EP367 / 225. CARACTÉRISATION DE 25 NOUVELLES MUTA- TIONS CHEZ 94 PROBANDS DE GRANULOMATOSE SEPTIQUE CHRONIQUE}

C. Kannengiesser (1), B. Gérard (1), J. El Benna (2), D. Henri (1), S. Chollet-Martin (2, 3), M.-A. Gougerot-Pocidalo (2, 3), C. Elbim (2, 3), B. Grandchamp (1)

(1) Service de Biochimie Hormonale et Génétique, Hôpital Bichat-Claude Bernard, 46 rue Henri Huchard, 75018 Paris, France; (2) INSERM U773, Faculté Xavier Bichat, 16 rue Henri Huchard, 75018 Paris, France ; (3) Service d'Immmunologie et d'Hématologie, Hôpital Bichat-Claude Bernard, 46 rue Henri Huchard, 75018 Paris, France

Introduction : La granulomatose septique chronique (chronic granulomatous disease, $\mathrm{CGD}$ ) résulte de mutations inactivatrices constitutionnelles de l'un des 4 gènes $C Y B B, C Y B A, N C F 1$ et $N C F 2$ codant respectivement pour les sous-unités de la NADPH oxydase, gp91phox, p22phox, p47phox et p67phox. Les mutations de $C Y B B$, gène localisé sur le chromosome $\mathrm{X}$ sont retrouvées dans la forme la plus fréquente transmise sur le mode récessif lié à I'X. Les autres formes sont transmises sur le mode autosomique récessif. Matériel et Méthodes: Une série de 94 cas index (88 hommes et 6 femmes) ont été diagnostiqués comme CGD sur des critères cliniques et biologiques (absence de production de formes réactives de l'oxygène par les polynucléaires neutrophiles); l'identification de la sous unité manquante a été réalisée par immunoblot. En fonction des résultats, une recherche de mutations dans les séquences codantes, les jonctions exons-introns de $C Y B B, C Y B A, N C F 1$ ou $N C F 2$ a été réalisée par séquençage direct. Des marqueurs microsatellites furent étudiés pour certaines mutations récurrentes de $C Y B B$. Résultats : l'exploration moléculaire de 93 probands sur 94 ( 88 hommes et 6 femmes) s'est révélée positive. Soixante seize ( 73 hommes et 3 femmes) étaient porteurs d'une mutation de $C Y B B$. Les 3 femmes présentaient un biais d'inactivation de l'X. Douze patients portaient une mutation de $N C F 1$ : 9 hommes homozygotes pour la mutation récurrente delGT et 3 femmes (dont 2 homozygotes pour deux mutations non sens distinctes et 1 hétérozygote composite pour la delGT et une mutation non sens). Trois patients présentaient une mutation de $N C F 2$ et 2 patients une mutation de $C Y B A$, tous à l'état homozygote. Parmi 67 mutations différentes identifiées, 30 n'ont jamais été rapportées à notre connaissance: 25 nouvelles mutations de $C Y B B$ dont 5 mutations faux sens, 5 mutations affectant les sites d'épissage, 9 mutations non sens, 3 délétions et 1 duplication décalant le cadre de lecture, 6 grandes délétions. Nous décrivons aussi une nouvelle mutation non sens de NCFI, une nouvelle mutation affectant les sites d'épissage et une délétion pour $N C F 2$, de même que pour CYBA. Les données expérimentales (analyse fonctionnelle des polynucléaires neutrophiles et immunoblot) montrent que ces mutations diminuent fortement ou abolissent l'expression de la sous unité en cause et la fonction de la NADPH oxydase. L'analyse des microsatellites montrent que 5 mutations de $C Y B B$ qui constituent des mutations récurrentes, rapportées plusieurs fois dans les bases de données et plusieurs fois dans notre série (supérieur ou égal à 2) correspondent à des points chauds mutationnels. Discussion: Cette série confirme la grande hétérogénéité génétique des CGD. Pour les formes de CGD non liées à l'X, on retrouve une homozygotie vraie dans 16 sur 17 cas, traduisant une fréquente consanguinité dans ces formes. Les CGD liées à l'X sont les formes génétiques les plus fréquentes $(82 \%)$ dans notre série. Cette proportion est supérieure à celle de $70 \%$ rapportée dans la plus grande série publiée (États-Unis : 368 patients, Winkelstein et al., 2000). Notre recrutement comprend probablement moins de familles consanguines. Une autre observation est le faible nombre de cas index de sexe féminin dans les formes récessives ( $3 / 17$ cas, $p=0.012)$, suggérant qu'il puisse exister un sousdiagnostic de la granulomatose septique chronique chez les filles. Il est intéressant à cet égard de noter que nous retrouvons chez 2 parmi les 3 filles ayant une forme récessive avec mutations de $N C F 2$ un tableau clinique atténué.

Mots-clés : granulomatose septique chronique, CYBB, NADPH oxydase.

"P368 / 226. DÉTECTION D'ÉLÉMENTS EXONIQUES RÉGULATEURS D'ÉPISSAGE ET IDENTIFICATION DES PROTÉINES IMPLIQUÉES DANS CETTE RÉGULATION : L'EXEMPLE DES GËNES MMR DANS LE SYNDROME DE LYNCH

M. Vezain (1), J. Soret (2), J. Tazi (2), T. Frébourg (1, 3), M. Tosi (1) A. Martins (1)

(1) Inserm U614, Faculté de Médecine et de Pharmacie, Institut Hospitalo-Universitaire de Recherche Biomédicale de Rouen; (2) Institut de Génétique Moléculaire de Montpellier (IGMM), CNRS UMR 5535, Montpellier: (3) Service de Génétique, CHU de Rouen

De nombreux variants de signification biologique inconnue sont identifiés chez les patients, dans le cadre de l'analyse de gènes impliqués dans les maladies mendéliennes. De tels variants ne peuvent pas être utilisés sur le plan médical. Certains de ces variants pourraient avoir un effet sur la maturation de l'ARN pré-messager en altérant des éléments exoniques de régulation d'épissage, tels que les ESE (Exonic Splicing Enhancers) ou les ESS (Exonic Splicing Silencers). Les prédictions informatiques de l'effet des mutations sur ces séquences régulatrices d'épissage ne sont pas fiables car ces séquences sont actuellement peu caractérisées. Grâce au développement d'un test d'épissage ex vivo, nous avons pu reclasser, en mutations d'épissage, certains variants identifiés dans les gènes $M L H 1$ et $M S H 2$ impliqués dans le cancer colorectal héréditaire non polyposique ou syndrome de Lynch (voir résumé d'I. Tournier et coll). En particulier, nous avons identifié dans les exons 10 et 11 de $M L H 1$ et dans l'exon 5 de $M S H 2$ des mutations situées à distance des sites d'épissage et provoquant des sauts d'exon, ce qui suggère qu'elles affectent des séquences ESE et/ou qu'elles créent des séquences ESS. Dans le but d'identifier les séquences ESE présentes dans les gènes $M L H 1$ et $M S H 2$ et de mieux comprendre le mécanisme des anomalies d'épissage associées au syndrome de Lynch, nous avons développé un test ex vivo indicateur d'ESE. Ce test est basé sur l'analyse des produits d'épissage d'un minigène portant un exon central ESE-dependant dans lequel sont insérées de courtes séquences exoniques d'intérêt, de l'ordre de $30 \mathrm{pb}$. Les minigènes sont alors transfectés dans 
des cellules HeLa et les produits d'épissage sont analysés par RT-PCR et séquençage. Nous avons utilisé ce test pour cribler toutes les régions exoniques de $M L H 1$ ou de $M S H 2$ soupçonnées de contenir des éléments régulateurs d'épissage. Nos résultats montrent que toutes les séquences testées induisent effectivement l'inclusion de l'exon central dans le transcrit du minigène et qu'elles correspondent effectivement à des ESE. Ainsi, nous avons défini 12 régions ESE, d'environ 30 nucléotides, dans les gènes MMR : 8 dans $M L H 1$ et 4 dans $M S H 2$. Nous avons constaté que, parmi les 15 mutations d'épissage touchant ces séquences et détectées dans des familles présentant un syndrome de Lynch, 10 ont également un effet négatif sur l'épissage dans le contexte du test ESE-dépendant. Plusieurs facteurs agissant en trans ont été impliqués dans la régulation de l'épissage, notamment les protéines SR (serine-arginine rich) et les protéines hnRNP (heterogeneous nuclear ribonucleoproteins). Nous avons alors entrepris la recherche des protéines capables de reconnaître spécifiquement les 12 séquences exoniques régulatrices d'épissage de $M L H 1$ et $M S H 2$ et de moduler l'épissage. Nous analysons actuellement les interactions ARNprotéine qui se produisent lorsque des ARNs transcrit in vitro correspondants aux 12 séquences exoniques, sauvages ou mutées, sont incubés avec des extraits nucléaires de cellules HeLa. Les interactions ARN-protéine sont étudiées par des tests tels que le retard sur gel, le pontage UV, l'immuno-marquage ou la chromatographie d'affinité. Ces approches nous ont déjà permis de mettre en évidence certains complexes protéiques qui reconnaissent différemment les régions ESE en version sauvage et mutée. Nous envisageons de définir la composition de ces complexes et d'utiliser cette information pour développer des modèles de correction in vitro et en culture cellulaire de ces épissages défectueux.

Mots-clés : épissage, ESE, syndrome de Lynch.

IP369 / 228. NOUVELLE MUTATION DU GÈNE DE L'ENDOGLINE DANS LA MALADIE DE RENDU OSLER

M. Borsik (1), C. Gourme (2), J. Callebert (3), P. Tran Ba Huy (1), L. Drouet (2), J.-M. Launay (3), K. Peoc'h $(2,3)$

(1) Service d'ORL, Hôpital Lariboisière, 2, rue Ambroise Paré, 75475 Paris cedex 10, France; (2) Service d'Hématologie Biologique, Hôpital Lariboisière; (3) Service de Biochimie et Biologie Moléculaire, Hôpital Lariboisière

La maladie de Rendu-Osler ou télangiectasie hémorragique héréditaire (HHT) se traduit par une dilatation permanente de petits vaisseaux de la peau et des muqueuses, appelées télangiectasies. Elle peut se compliquer d'hémorragies, d'anémie et de fistules artério-veineuses surtout hépatiques, pulmonaires ou au niveau du système nerveux central, et se manifeste fréquemment de manière initiale par des saignements de nez importants et répétés, parfois dès l'enfance. Cette maladie autosomique dominante touche moins de 10000 personnes en France. La pénétrance est élevée et l'expressivité variable, même si la maladie est symptomatique chez presque tous les patients de plus de 40 ans. Elle est principalement associée à des mutations du gène de l'endogline (HHTl, chr 9q33-34, OMIM : 187300) ou de «l'activine-like kinase receptor» (HHT2, chr 12q13, OMIM : 600376). Ces deux protéines sont impliquées dans la transduction du signal $\mathrm{du} \mathrm{TGFb}$, qui joue un rôle important dans la formation du système vasculaire. Nous décrivons une famille de patients atteints de maladie de RenduOsler pour lesquels une insertion d'une base $t$ a été détectée à l'état hétérozygote dans l'exon 2 du gène de l'endogline (p. Val66Cys fx X148, c. ins 195-196 t). Le propositus, une femme de 37 ans, a présenté des épistaxis dès l'enfance puis des angiomes linguaux et labiaux, suivis de fistules artério-veineuses pulmonaires ayant nécessité des embolisations successives. Les patients de cette famille atteints de la maladie présentent la mutation, les sujets sains ne présentent pas cette anomalie. Cette insertion entraîne l'apparition d'une forme tronquée de la protéine qui passe de 625 à 147 acides aminés. Il a été montré dans la littérature que les formes tronquées de l'endogline étaient associées soit à des anomalies du signal liée à une dimérisation anormale, soit à une sécrétion dans le milieu extracellulaire de ces glycoprotéines transmembranaires. Plus de 140 mutations de l'endogline ont été décrites à ce jour ; il s'agit à notre connaissance de la première description de cette mutation.

Mots-clés : Rendu Osler, endogline, télangiectasie.

DP370 / 229. VKORC1 ET CYP2C9 DANS LA SENSIBILITÉ AUX ANTI-VITAMINE K : À PROPOS D'UN PATIENT

K. Peoc'h, C. Bal, S. Pruvot, C. Gourmel, S. Thomas, D. Paulin, L. Drouet Service d'Hématologie Biologique, Hôpital Lariboisière, 2 rue Ambroise Paré, 75475 Paris cedex 10

Les antivitamines K (AVK) coumariniques (Warfarine, Acenocoumarol) ou indanedione (Fluindione) sont le seul traitement anticoagulant par voie orale disponible dans le traitement et la prévention des récidives des événements thromboemboliques. Ces deux familles exercent leur activité anticoagulante en inhibant le complexe « vitamin $\mathrm{K}$ epoxide reductase multiprotein " (VKORC1). Ce complexe recycle la vitamine K 2,3-epoxide en vitamine $\mathrm{K}$ hydroquinone, un cofacteur essentiel pour la g-carboxylation et donc la fonctionnalité de plusieurs facteurs inhibiteurs de la coagulation. Le traitement est rendu difficile par la nécessité d'une adaptation rigoureuse des doses et par une très grande variabilité interindividuelle de réponse, sous tendue par des facteurs génétiques et environnementaux en particulier médicamenteux et nutritionnels. Des polymorphismes du gène d'un isoforme du cytochrome, notamment le CYP2C $9 * 2$ (p.Arg144Cys), ont été associés à une faible métabolisation des coumariniques et des indanediones. Des polymorphismes du gène $V K O R C I$ ont été associés à une différence de sensibilité de VKORC1 aux AVK. Les phénotypes par associations des polymorphismes de VKORC1 et CYP2C9 expliquent près de $50 \%$ de la variabilité individuelle de sensibilité aux AVK. Plus récemment des mutations du gène $V K O R C I$ ont été découvertes chez des patients constitutionnellement résistants aux AVK. Il s'agit du mécanisme le plus fréquent de résistance aux coumariniques utilisés comme raticides chez les rongeurs. Nous rapportons l'histoire d'un patient antillais, âgé de 38 ans, présentant une résistance clinique et biologique aux AVK. Ce patient, hétérozygote pour la mutation du facteur V Leiden, a présenté un épisode de thrombose veineuse profonde compliquée d'une embolie pulmonaire. Son INR est resté proche de 1 malgré une augmentation des doses de fluindione puis de warfarine à plus de 4 fois les doses habituellement efficaces ( $>40 \mathrm{mg} /$ jour de warfarine). L'étude du gène $V K O R C l$ a permis d'identifier l'existence d'une mutation du codon 68 portée à l'état hétérozygote dans l'exon 2 (p.His68Tyr, c. $202 \mathrm{c}>\mathrm{t}$ ). Cette mutation, vient d'être décrite dans une population suédoise, mais n'avait pas été associée à une résistance aux AVK (Osman et al., J Thromb. Haemost., 2006). De manière surprenante, ce patient présentait également le polymorphisme CYP2C $9 * 2$ à l'état hétérozygote. Ce polymorphisme est associé à un phénotype de faible métaboliseur de la Warfarine et par conséquent à un risque accru de surdosage aux AVK (Rettie et al., Pharmacogenetics, 1994). Cette description met en évidence l'impact majeur des mutations de VKORC1 sur la sensibilité aux AVK, et ce même en présence de polymorphismes *2 du CYP2C9. L'analyse combinée des gènes $V K O R C 1$ et $C Y P 2 C 9$ aide à identifier les sensibilités constitutionnelles aux AVK, même si d'autres gènes comme la g-carboxylase jouent également un rôle. La pharmacogénétique est très utile pour dépister les sensibilités et la résistance aux AVK et mieux prendre en charge le traitement des patients pour maladie thromboembolique.

Mots-clés : VKORC1, CYP2C9, pharmacogénétique.

EP371 / 234. HYPEROXALURIE DE TYPE I : IDENTIFICATION DE NOUVELLES MUTATIONS DU GENE AGXT DANS UNE COHORTE DE 111 MALADES

F. Chevalier-Porst, G. Souche, M.-O. Rolland, I. Maire, R. Froissart

Service Maladies Héréditaires du Métabolisme, Centre de Biologie et Pathologie Est-5 $5^{e}$ étage Groupement Hospitalier Est BRON

Introduction : L'hyperoxalurie de type I (HPI) révélée par des urolithiases et/ou une néphrocalcinose est une maladie transmise selon le mode autosomique récessif. Elle est due à un déficit en Alanine glyoxylate amino transférase (AGT) localisée dans les péroxysomes du foie. Le diagnostic biologique repose sur la mise en évidence d'une excrétion constante d'oxalate associée dans $2 / 3$ des cas à une excrétion de glycolate. Le gène $A G X T$, localisé sur le chromosome 2, comprend 11 exons. Chez la plupart des patients l'identification des mutations permet maintenant d'éviter la ponction d'une biopsie de foie pour mesurer l'activité de l'AGT. 55 altérations de ce gène ont déjà été identifiées; 4 mutations fréquentes représentent environ $40 \%$ des allèles mutés, la plupart des autres mutations sont peu fréquentes ou privées. Le polymorphisme P11L permet d'identifier un allèle majeur $(\mathrm{P} 11)$ et un allèle mineur $(\mathrm{L} 11)$; l'allèle mineur est retrouvé chez plus de $60 \%$ des patients atteint d'HP1 contre $15-20 \%$ dans la population générale. Matériel et Méthode : L'étude mutationnelle du gène AGXT a été réalisée par séquençage des 11 exons et de leur jonctions intron exon chez 111 patients non apparentés. Le bornage de grandes délétions a été effectué par PCR en temps réel ou par études familiales (par séquençage) de zones introniques polymorphes. Résultat et Discussion : Le génotype complet de 96 des 111 patients a été déterminé. Parmi les 52 altérations différentes retrouvées, les mutations les plus fréquentes sont: G170R $(17.6 \%)$ et 1244T $(18 \%)$ toujours associées à l'allèle mineur, c.33_34insC $(7.6 \%)$ toujours associée à l'allèle majeur. Vingt-trois altérations nouvelles, 2 polymorphismes exoniques ont été identifiés. Trois délétions de plusieurs exons ( 3 à 7 exons délétés) ont été bornées. Chez 5 patients, une seule mutation a été identifiée. Le diagnostic d'HP I a pu être confirmé

(1)


par la mise en évidence du déficit enzymatique dans le foie chez trois d'entre eux, dans les deux autres cas, seule la clinique et l'augmentation de l'excrétion urinaire d'oxalate orientent vers le diagnostic. Conclusion : Compte tenu de la petite taille du gène de l'AGXT, le séquençage est une méthode adaptée à la confirmation du diagnostic d'hyperoxalurie de type I. La détermination de l'activité enzymatique ne devient alors indispensable, pour confirmer ou exclure le diagnostic, que dans quelques rares cas. Enfin l'étude mutationnelle permet de proposer un diagnostic prénatal aux couples à risque.

Mots-clés : hyperoxalurie de type I, mutations, génotype.

IP372 / 236. SYNDROME DE TAYBI-LINDER : MAIS OÙ SE CACHE LE GENE ?

A. Labalme (1), P.S. Jouk (2), A. Toutain (3), E. Steichen (4), R. Touraine (5), A. L. Leutenegger (6), E. Genin (6), F. Clerget-Darpoux (6), P. Edery $(1,7)$

(1) Service de Cytogénétique Constitutionnelle, $\mathrm{CHU}$ de Lyon ; (2) Service de Génétique Pédiatrique, CHU de Grenoble; (3) Service de Génétique, CHU de Tours ; (4) Service de Génétique, CHU d'Innsbuck; (5) Laboratoire de Biologie Moléculaire, CHU de Saint-Étienne; (6) Laboratoire d'Épidémiologie Génétique, INSERM U535; (7) Service de Génétique Pédiatrique, $\mathrm{CHU}$ de Lyon

Le Nanisme Primordial Microcéphalique Ostéodysplastique de type I/III (MOPD type I/III, OMIM 210710), ou syndrome de Taybi-Linder, est un syndrome autosomique récessif très rare de cause encore inconnue. Il est caractérisé par un retard de croissance intra-utérin sévère, une hypotrophie, un nanisme et des anomalies osseuses. Les patients présentent également un faciès typique, avec une peau sèche et des cheveux et sourcils épars, une microcéphalie et de sévères malformations cérébrales (Taybi and Linder 1967; Majewski and Spranger 1976). Ils décèdent souvent dans la première année de vie. Nos premiers travaux sur le syndrome de TaybiLinder ont permis de localiser le gène responsable sur le chromosome 2 q14 (lod score Zmax $=3.85$, Leutenegger $\mathrm{et}$ al. Am J Hum Genet 2006). Cependant, notre analyse n'incluait qu'un petit nombre de patients (4 patients appartenant à 3 familles non apparentées) ne permettant pas d'exclure totalement l'hypothèse d'une hétérogénéité génétique de ce syndrome. Aujourd'hui, l'étude d'une nouvelle patiente est en faveur de l'homogénéité génétique du syndrome de Taybi-Linder. Cette patiente, d'origine Marocaine, issue de parents cousins germains présente un phénotype très similaire à celui des autres patients inclus dans notre première étude. Après génotypage fin de cette patiente et de ses parents avec un grand nombre de marqueurs du chromosome $2 \mathrm{q} 14$, l'analyse des haplotypes a révélé une région autozygote sur 9 marqueurs microsatellites consécutifs permettant de réduire notre intervalle candidat à $3.2 \mathrm{Mb}$. Le séquençage direct des séquences codantes et des séquences introniques flanquantes de tous les gènes annotés de cette région (NCBI, Ensembl) s'est avéré normal et n'a donc pas permis, à ce jour, d'identifier le gène responsable. Ces résultats permettent maintenant de proposer un diagnostic prénatal moléculaire indirect de cette affection très sévère, dans les familles étudiées, sur la base de l'étude des haplotypes de la région critique du chromosome 2 q14.

Mots-clés : Taybi-Linder, homogénéité génétique, diagnostic prénatal.

IP373 / 238. BASES MOLÉCULAIRES DES FORMES DOMINANTES ET RÉCESSIVES DES MYOPATHIES CONGÉNITALES STRUCTURALES À CORES ASSOCIÉES AU GÈNE RYRI

N. Monnier (1, 2), I. Marty (2, 3), J. Faure (1, 2, 3), C. Castiglioni (4), S. Sacconi (5), B. Estournet (6), A. Ferreiro (7), N. Romero (7), A. Laquerriere (8), L. Lazaro (9), J.-J. Martin (10), E. Morava (11), A. Rossi (12), A Van der Kooi (13), C. Verschuure (14), J. Lunardi $(1,2,3)$

(1) Laboratoire de Biochimie et Génétique Moléculaire et Centre de Référence des Maladies Neuro-Musculaires, CHU Grenoble, 38043, France; (2) INSERM, U836, Grenoble Institut des Neurosciences, 38043, Grenoble, France ; (3) Université Joseph-Fourier, 38041, Grenoble, France ; (4) Servicio di Neurologia, Clinica Las Condes, Santiago, Chile (5) Fédération des Maladies Neuromusculaires, CHU Nice, France; (6) Hôpital Raymond-Poincaré, Garches, France ; (7) Institut de Myologie et INSERM U582, Paris, France; (8) Service d'Anatomie et Cytologie Pathologique, CHU Rouen, France; (9) Département de Biochimie et Génétique Moléculaire, CHU Rennes, France; (10) Instituut Born-Bunge, Universiteit Antwerpen, Belgium ; (11) Department of Pediatrics, University Children's Hospital, Nijmegen, The Netherlands; (12) CHU-Rouen, France; (13) Academisch Medisch Centrum, Amsterdam, The Netherlands; (14) Clinical Genetics, University Medical Center, Groningen, The Netherlands

Introduction : Les myopathies congénitales structurales à cores regroupent ou à multiminicores $(\mathrm{MmD})$. Ces pathologies sont causées par des mutations du gène codant l'isoforme musculaire du récepteur de la ryanodine (RYRI) responsable d'un dysfonctionnement de l'homéostasie calcique dans la fibre musculaire squelettique. Ces pathologies sont caractérisées par une forte hétérogenéité clinique, histologique et génétique qui rend parfois difficile l'utilisation des critères nosologiques actuels définis essentiellement sur la base des observations morphologiques. Méthodes : Nous avons étudié un panel de 241 propositus originaires de familles indépendantes et inclus sur la base d'un diagnostic de myopathie à cores à l'exclusion des autres pathologies associées au gène RYRI telles qu'hyperthermie maligne, hyperthermie d'effort... Le gène RYRI a été exploré au niveau génomique par analyse ciblée des 15 exons du domaine C-terminal transmembranaire et par séquençage de la totalité du transcrit lorsque cela a été possible ( 68 cas). La présence de la protéine dans le muscle des patients a été évaluée par immunodécoration. Résultats-Conclusions : L'analyse morphologique des biopsies musculaires a montré que les lésions de type cores se distinguaient par une variabilité en terme de localisation, taille, longueur, aspect et nombre. Cent-cnquante-cinq propositus étaient des cas sporadiques tandis que 74 cas se sont révélés être des formes dominantes et que 12 patients appartenaient à des formes récessives. Nous avons identifié une mutation dominante du gène $R Y R I$ chez 65 patients, deux mutations récessives chez 30 patients et une seule mutation chez 20 autres cas de statut $\mathrm{AR}$ ou $\mathrm{AD}$ non déterminé. Les mutations dominantes sont des mutations faux-sens ou des délétions en phase principalement localisées dans la partie Cter de la protéine. Par contraste, les 60 mutations identifiées dans les formes récessives se répartissent tout au long de la séquence codante avec $25 \%$ de mutations nulles. Deux patients présentant une forme néonatale sévère étaient porteurs de deux mutations conduisant à une perte quasi complète de l'expression de RYRI. Douze autres patients étaient porteurs d'une mutation nulle et d'une mutation faux sens. Dans ces situations la sévérité du phénotype clinique est vraisemblablement associée à la nature de la mutation faux-sens. De manière intéressante, nous avons mis en évidence un nouvel exon alternatif présent dans les formes récessives les plus sévères. Ces données seront discutées en terme de relation génotype-phénotype, elles montrent également que la présence d'une seconde mutation doit être recherchée dans les formes sporadiques ou dans les formes dominantes montrant une variabilité clinique intrafamiliale. Les données obtenues montrent clairement l'existence d'un continuum morphologique et clinique entre les formes dominantes classiques de type CCD et les formes récessives de type $\mathrm{MmD}$.

Mots-clés : myopathies congénitales, récepteur de la ryanodine, calcium.

\section{- 374 / 254. PRÉSENCE, DANS UNE LAM4, D'UN VARIANT INHABITUEL ISSU PROBABLEMENT D'UNE FUSION CBFB- MYH11 TYPE A PAR ÉPISSAGE ALTERNATIF SIMULTANÉ DE CBFB ET MYHII}

A.N. Nguyen (1), B. Leotard (1), N. Perrard (1), C. Robquin (1), C. Bonmati (2), B. Witz (2), S. Salignac (3), M.J. Grégoire (1), P. Jonveaux (1) (1) Laboratoire de génétique, CHU Nancy; (2) Hématologie médecine interne, CHU Nancy: (3) Laboratoire d'hématologie-CHU Nancy

Le diagnostic de LAM M4FAB sans composante éosinophile est porté au myélogramme chez une patiente de 71 ans sans antécédent oncologique particulier, à la suite d'un hémogramme hypercytaire (GB 285G/l) largement infiltré par la lignée monocytaire (blastes $25 \%$, monocytes et promonocytes $73 \%$ ). Le caryotype médullaire retrouve la présence d'une inversion du chromosome 16 récurrente dans ces LAM4; L'hybridation in situ confirme la cassure au locus CBFB résultant de cette inversion. L'analyse moléculaire par RT-PCR (BIOMED1) montre la bande attendue dans le réarrangement de type A (418 pb), ainsi qu'une bande supplémentaire plus grande ( $495 \mathrm{pb})$. L'une des séquences correspond au transcrit de fusion CBFB nt $474 /$ MYH11 nt1921 de type A, retrouvé dans $85 \%$ à $90 \%$ des inversions du 16. La séquence du produit de PCR de $495 \mathrm{pb}$ résulte, quant à elle, d'une fusion CBFB nt505/MYH11 nt1874, transcrit non décrit à notre connaissance à ce jour. Le réarrangement sur $M Y H 11$ entre nt1874 et nt1921 (47nt) peut correspondre à un épissage alternatif de ce gène ; pour le passage de nt474 à nt505 de $C B F B$ (3lnt) un tel mécanisme est mis en cause ; il interromprait le cadre de lecture de la protéine CBFB ; celui-ci est rétabli par l'épissage de $M Y H 11$, comme cela est suggéré pour un autre variant inhabituel (van der Reijden BA, blood,1995,86,277-281). Il a été décrit que des variations de la protéine de fusion peuvent perturber l'efficacité de la formation du complexe hétérodimérique avec la protéine AML1 et peut être, son action leucémogène ainsi que la présentation diagnostique de la leucémie. (Schnittger S, Leukemia,2007,21,725-731) Il est à noter que notre patiente présentait une LAM4 de novo, hypercytaire sans composante eosinophile anormale, CD13,CD34,CD33,MPO,CD11c,CD14 
positifs ; elle a bien répondu au traitement d'induction réalisé selon le protocole GOELAMS et est actuellement en rémission cytologique.

Mots-clés : variants CBFB/MYH11, épissage alternatif, inv(16).

\section{IP375 / 258. MISE AU POINT D'UNE PUCE DE RESÉOUENCAGE DE COL3A1 POUR LE DIAGNOSTIC DU SYNDROME D'EHLERS DANLOS VASCULAIRE}

S. Vonwill (1), N. Le Pottier (1), J.C. Haw-King-Chon (2), J. Perdu (1, 3), X. Jeunemaître $(1,3)$

(I) Service de Génétique, Hôpital Européen Georges-Pompidou, Paris ; (2) Plate-forme de génomique fonctionnelle, Institut Curie, Paris; (3) Centre de référence des Maladies Vasculaires Rares, Hôpital Européen Georges-Pompidou, Paris

Introduction: Le Syndrôme d'Ehlers Danlos vasculaire (SEDv, OMIM $\# 130050$ ) est une pathologie héréditaire autosomique dominante du tissu conjonctif liée à des mutations du gène $C O L 3 A 1$, codant pour la chaîne alphal du collagène de type III. Il se caractérise par des complications vasculaires, gastro-intestinales et/ou obstétricales. Compte tenu de la variabilité du phénotype, seule la détection d'une anomalie du collagène III, au niveau biochimique et/ou génétique, certifie le diagnostic. Le test génétique classique consiste en l'analyse du cDNA de COL $3 A 1$ après biopsie de peau et culture de fibroblastes. Cette analyse est relativement lourde et coûteuse. Le délai d'obtention des résultats ( $>3$ mois) et sa sensibilité imparfaite (larges délétions non détectées) justifient le développement d'autres approches diagnostiques. Une stratégie alternative est le séquençage direct de l'ensemble des parties codantes de COL3Al (5490bp). Cette technique est lourde ( $n=51$ exons) et peut aussi manquer d'éventuelles délétions partielles ou des mutations dans les régions ' $5^{\prime}$ et $3^{\prime}$ du gène. Notre projet, financé par le Ministère de la Santé (DHOS, STIC 2006), a pour but d'évaluer et de comparer les performances de ces deux techniques à celle de l'utilisation d'une puce de reséquençage de $C O L 3 A /$ sur matériel Affymetrix. Cette méthodologie repose sur l'analyse directe de l'ensemble du gène $C O L 3 A I$ à partir de sang périphérique. Nous décrivons ici la mise en place de cette technologie et ses premiers résultats pour 24 patients. Méthodes : En collaboration avec le «Chip design » d'Affymetrix, nous avons configuré une puce de reséquençage $(50 \mathrm{~kb})$ à façon, telle que la séquence complète de COL3A1 (ENSG00000168542) soit représentée. Afin d'éviter les hybridations aspécifiques, nous avons écarté les séquences homologues et répétées. Ainsi, 35782 bp de COL $3 A 1$ (dont 115 bases en 5' et 110 en 3'), réparties en 25 séquences indépendantes, peuvent être étudiées. Nous avons ajouté 19 séquences de 25 bp portant des SNPs de COL $3 A 1$. À partir de l'ADN périphérique, des protocoles $\mathrm{PCR}$ «long range » permettent d'obtenir trois amplicons de $10 \mathrm{~kb}$ et un de $8,9 \mathrm{~kb}$, couvrant l'ensemble du gène. Ces amplicons sont traités à la DNasel pour former des fragments de 20 à $200 \mathrm{bp}$, puis hybridés sur la puce une nuit à $49^{\circ} \mathrm{C}$, en conditions équimolaires. Après lavage, l'hybridation est traduite par les logiciels GCOS et GSEQ (Affymetrix) en séquences nucléotidiques. Nous avons développé un programme informatique permettant de réordonner les 25 séquences, afin de lire l'ensemble du gène $C O L 3 A 1$ dans le sens $5^{\prime}-3$ '. Résultats: Nos résultats préliminaires portent sur 7 patients sans mutations connues de COL $3 A$ I . Des données supplémentaires portant sur 16 individus mutés (contrôles positifs) seront présentées. L'analyse a été possible pour 6 patients sur 7. Elle a permis de lire en moyenne 92,6\% ( «Call Rate » ou CR) de la séquence représentée sur la puce et $93,8 \%$ des séquences exoniques. Par ailleurs, 207 SNPs répartis sur tout le gène $C O L 3 A l$ peuvent être étudiés. Après analyse, $10 \mathrm{SNPs}$ sont indéterminés pour les 6 individus; et la moyenne des CR des 207 SNPs est de $89 \%$. Toutefois, l'étude en parallèle des séquences additionnelles, qui renvoient à 19 de ces SNPs, révèle 2 discordances. Conclusion : Les résultats actuels montrent la faisabilité de cette technologie, mais des nécessités de mise au point et des difficultés techniques d'interprétation devront être résolues avant l'utilisation de cette puce en pratique courante. Par ailleurs, les coûts de la technologie seront estimés. En fonction du rapport coût/avantages, et de l'impact financier de la poursuite de la technologie, il sera possible, dans un second temps, d'élargir la population à tester.

Mots-clés : COL3A1, puces ADN, reséquençage.

IP376 / 265. CARACTÉRISATION DE DÉLETIONS DE SOX10 DANS LE SYNDROME DE WAARDENBURG-HIRSCHSPRUNG

N. Bondurand (1), F. Dastot-Le Moal $(1,2)$, V. Baral $(1)$, N. Collot $(1,2)$, I. Giurgea (1, 2), P. Sarda (3), A. Echaieb (4), R. Touraine (5), J. Amiel (6), M. Goossens $(1,2)$, V. Pingault $(1,2)$

(1) INSERM U841, IMRB, Département de génétique, Créteil, France : (2) AP-HP, Groupe Henri-Mondor-Albert-Chenevier, Service de biochimie et génétique, Créteil, France ; (3) Depatment of Medical genetics, CHU Montpellier, France; (4) Chirurgie pediatrique, Hôpital
Pierre-Zobda-Quitman, Fort de France, France ; (5) CHU-Hôpital Nord Service de Génétique Saint-Étienne ; (6) INSERM U781 et département de Génétique, Hôpital Necker, Paris, France

Le facteur de transcription SOX10 joue un rôle essentiel au cours du développement de certains systèmes ou lignages dérivés de la crête neurale, et notamment du système nerveux entérique (SNE), des mélanocytes, et des cellules de Schwann impliquées dans la formation de la myéline du système nerveux périphérique. En accord avec ces observations, une trentaine de mutations de SOX1O ont été décrites depuis 1998, chez des patients présentant une aganglionose intestinale plus ou moins étendue ainsi que des anomalies de pigmentation et une surdité, une association connue sous le nom de syndrome de Waardenburg-Hirschsprung ou syndrome de Waardenburg de type 4 (WS4). Des signes neurologiques associés ont également été rapportés dans certains cas, conduisant à la définition d'un syndrome appelé PCWH (Peripheral demyelinating neuropathy-Central dysmyelinating leukodystrophy-Waardenburg syndrome-Hirschsprung disease). Les mutations identifiées jusqu'à présent sont principalement des mutations ponctuelles tronquantes, et plusieurs arguments indiquent que la plus grande sévérité du phénotype PCWH survient lorsque l'ARNm mutant échappe au mécanisme de surveillance NMD (nonsense mediated decay). En plus de $S O X 10$, des mutations au sein des gènes codant le récepteur à sept domaines transmembranaires EDNRB et son ligand l'endothéline 3 (EDN3) ont été observées chez 20 à $30 \%$ des patients WS4. Cependant, tous les cas n'ont pas trouvé d'explication moléculaire, conduisant à penser que d'autres gènes puissent être impliqués ou que certaines mutations au sein des gènes connus ne sont pas détectées par les méthodes de génotypage usuelles. Nous avons utilisé les techniques de PCR quantitative multiplex fluorescente et d'hybridation in situ fluorescente pour rechercher des délétions de SOX10. Nous avons ainsi identifié les 3 premières délétions hétérozygotes de SOX10 chez des patients présentant un WS4 ou un PCWH. La caractérisation de ces délétions a révélé différents évènements, allant de la délétion d'un seul exon à une délétion de $220 \mathrm{~Kb}$ incluant SOX10. De façon remarquable, l'un des patients présente de plus une substitution d'une valine en leucine à l'état hémizygote (Val92Leu). Les conséquences fonctionnelles de la variation Val92Leu, ainsi que l'influence de la délétion d'autres gènes autour de SOX10 sur la sévérité du phénotype, seront discutées.

Mots-clés : waardenburg-Hirschsprung, SOX10, délétion.

- 377 / 266. VARIATIONS INTRONIQUES À DES POSITIONS PEU CONSERVÉES DANS LES GẼNES USH2A ET MYO7A : QUEL EFFET SUR L'ÉPISSAGE ?

S. Le Guédard-Méreuze (1), C. Vaché (2), J. Vaudaine (1), L. Larrieu (2), V. Faugère (2), F.O. Desmet (1), D. Baux (2), M. Claustres (1, 2), A.F. Roux $(1,2)$, S. Tuffery-Giraud $(1,3)$

(1) INSERM, U827, Montpellier, France ; (2) CHU Montpellier, Montpellier, France ; (3) Université Montpellier 1, Montpellier, France

Introduction: L'interprétation de l'effet pathogène de variations de séquence touchant les sites d'épissage peut être difficile, en particulier lorsqu'elles impliquent des positions moins conservées que les dinucléotides obligatoires AG (site accepteur, 3'ss) et GT (site donneur, 5'ss) qui flanquent la plupart des exons. Cette étude a pour but d'élucider l'impact sur l'épissage de 7 variations introniques non classées identifiées dans le cadre du diagnostic du syndrome de Usher dans les gènes USH2A et MYO7A. Cinq d'entre elles sont situées aux positions $-3,-6,-8,-14$ et -17 du site 3 'ss, et deux à la position +3 du site 5 'ss. Toutes ces variations ont été trouvées en trans de mutations délétères et le séquençage complet du gène impliqué n'a révélé aucune autre variation potentiellement pathogène. Le but de cette étude est de déterminer la pathogénicité de ces variations. Matériels et méthodes: N'ayant pas accès aux transcrits spécifiques des gènes USH2A et MYO7A chez les patients, des tests fonctionnels ont été réalisés afin d'évaluer l'impact sur l'épissage de ces variations. Ces tests sont basés sur l'utilisation d'un minigène rapporteur fluorescent transfecté de façon transitoire dans des cellules HeLa. L'effet sur l'épissage est ensuite visualisé en microscopie à fluorescence et confirmé en RT-PCR et séquençage. L'impact de ces variations sur l'épissage a également été évalué grâce à différents algorithmes (Senapathy et Shapiro (CV), Maximum Entropy (MAXENT), Splice Site Prediction by Neural Network (SSPNN), Splice Sequence finder (SSF) d'une part et Free-Energy (Delta-G) et SD-Score pour les sites 5'ss). Les résultats des tests fonctionnels ont ainsi été comparés aux résultats obtenus in silico. Résultats et discussion: Les tests fonctionnels ont révélé une altération de l'épissage pour chacune des variations étudiées. Certaines sont à l'origine d'un skipping d'exon, d'autres impliquent l'utilisation de sites d'épissage de novo ou cryptiques. Nous avons par ailleurs observé que les algorithmes de prédiction donnant différents types d'informations, l'utilisation de 
plusieurs d'entre eux est souvent nécessaire pour évaluer l'impact sur l'épissage de variations introniques. On constate que si les outils bioinformatiques permettent en général de prédire assez bien une altération de l'épissage par une mutation (défaut d'utilisation du site naturel), seules des études de transcrits (chez le patients ou via des constructions de minigènes) permettent d'en évaluer les conséquences (saut d'exon, utilisation de sites cryptiques...). Conclusion : Cette étude montre une fois de plus la difficulté de prédire in silico l'impact de mutations d'épissage et renforce l'intérêt et la nécessité de réaliser des tests fonctionnels afin d'assigner un caractère pathogène à de telles variations non classées. Nous avons pu confirmer le rôle pathogène de chacune des variations testées, ce qui représente une aide importante pour le diagnostic des patients et la prise en charge des familles.

Mots-clés : mutations d'épissage, tests fonctionnels, syndrome de Usher.

\section{EP378 / 267. MISE EN ÉVIDENCE ET CARACTÉRISATION DE DÉLÉTIONS DE SOX10 DANS LE SYNDROME DE WAARDEN. BURG DE TYPE 2}

L. Stanchina (1), F. Dastot-Le Moal (1, 2), N. Collot (1, 2), V. Baral (1), S. Marlin (3), A. Toutain (4), W. Reardon (5), M. Lackmy-Port-Lis (6), R. Touraine (7), T. Attie-Bitach (8), M. Goossens (1, 2) V. Pingault (1, 2), N. Bondurand (1)

(1) INSERM U841, IMRB, Département de génétique, Créteil, France; (2) AP-HP, Groupe Henri-Mondor-Albert-Chenevier, Service de biochimie et génétique, Créteil, France; (3) Service de Génétique, INSERM U587, Hôpital Armand-Trousseau, APHP ; (4) Centre Hospitalo-Universitaire, Service de Génétique, Tours, France ; (5) Ladys Hospital for Sick Children, Dublin, Ireland; (6) Service de Pédiatrie, CHU de Pointe-à-Pitre, France-; (7) CHU-Hôpital Nord Service de Génétique; (8) Département de Génétique et INSERM U781, Hôpital Necker, Paris, France

Le syndrome de Waardenburg (WS) se caractérise par une surdité neurosensorielle et des anomalies de la pigmentation de la peau et des cheveux. Sur la base de l'association avec d'autres symptômes, le WS est divisé en quatre sous-types, WS1 à 4. L'absence de signes additionnels caractérise le WS2 tandis que l'association avec la maladie de Hirschsprung caractérise le WS4, également appelé syndrome de Waardenburg-Hirschsprung. Des mutations dans les gènes codant $M I T F$ et $S N A I 2$ ont été identifiées dans $15 \%$ des cas de WS2 et des mutations de EDN3, EDNRB et SOX10 ont été mises en évidence dans 60 à $80 \%$ des cas de WS4. Cependant, tous les cas n'ont pas trouvé d'explication moléculaire, laissant penser que d'autres gènes sont impliqués ou que certaines mutations au sein des gènes connus ne sont pas détectées par les méthodes de génotypage usuelles. Le rôle crucial de SOXIO au cours du développement mélanocytaire, et la variabilité phénotypique observée chez les patients présentant des mutations de ce gène, nous ont conduit à rechercher des mutations ou des délétions de SOX10 dans 30 cas non expliqués de WS2. Nous avons identifié 5 délétions hétérozygotes de $S O X 10$, ce qui en fait un nouveau gène impliqué dans le WS2. La caractérisation des délétions a révélé des évènements moléculaires indépendants, allant d'une délétion d'un seul exon à une délétion de plus de $5 \mathrm{Mb}$. Cette étude a des conséquences directes sur le diagnostic moléculaire, et démontre un peu plus la complexité moléculaire et les relations étroites qui lient les différents sous-types du syndrome de Waardenburg.

Mots-clés : WS2, SOX10, délétion.

口P379 / 278. LE DIAGNOSTIC MOLÉCULAIRE DANS LA DYSTROPHIE FACIO-SCAPULO-HUMÉRALE : ÉTUDE D'UNE COHORTE DE 42 PATIENTS

K. Nguyen (1, 2), J. Gallard (1), Cathy Vo Van (1), Rafaelle Bernard (1, 2), P. Malzac (1), B. Chabrol (3), J. Pouget (4), S. Attarian (4), N. Lévy $(1,2)$

(1) Département de Génétique Médicale, laboratoire de génétique moléculaire, CHU Timone Marseille; (2) UMR 910 Génétique Médicale et Génomique Fonctionnelle, faculté de Médecine Marseille; (3) Service de Pédiatrie, CHU Timone-Enfants Marseille; (4) Service de Neurologie, CHU Timone Marseille

Introduction : La dystrophie facio-scapulo-humérale (FSHD) est une dystrophie musculaire d'hérédité autosomique dominante associée à une délétion/contraction d'un nombre variable d'unités répétées (UR) en tandem de $3,3 \mathrm{~Kb}$ (D4Z4) dans la région sub-télomérique du chromosome $4 \mathrm{q} 35$. Le diagnostic de routine de la FSHD est réalisé par une technique de Southern-blot couplé à une électrophorèse linéaire (LGE) ou en champ pulsé (PFGE) utilisant la sonde p13e11 adjacente aux UR D4Z4 et une double digestion enzymatique EcoRI/BInI. Un allèle constitué de 1 à 10 UR pathogène, les allèles normaux étant porteurs de 11 à 100 UR. De plus, il existe un polymorphisme distal par rapport à la région $\mathrm{D} 4 \mathrm{Z} 4$, bi-allélique, avec deux variants $\mathrm{qA}$ et $\mathrm{qB}$. Seules les délétions/contractions couplées au polymorphisme qA sont associées à la FSHD. L'objectif de cette étude était d'explorer sur le plan moléculaire une large cohorte de patients atteints de FSHD et leurs apparentés sains afin de déterminer la taille des fragments D4Z4 et le typage qA/qB. Patients et Méthodes : les échantillons d'ADN de 42 sujets de 34 familles différentes ont été adressés au laboratoire de génétique moléculaire de Marseille pour diagnostic de FSHD. La technique de Southern-blot a utilisé plusieurs protocoles de digestion enzymatique : simple digestion EcoRI, double digestion EcoRI/BInI, simple digestion HindIII et simple digestion XapI, le LGE et le PFGE. Résultats : 4 allèles distincts n'ont pu être visualisés que chez 15 patients sur $42(36 \%)$. la visualisation des 2 allèles du chromosome 4 a été possible chez 33 patients sur $42(80 \%)$ et l'allèle délété a toujours été visualisé. La taille moyenne des allèles $4 \mathrm{q}$ contractés était de 28,69 kb ou 6,14 UR. De plus, nous avons mis en évidence des profils moléculaires atypiques et difficiles à interpréter : deux patients présentant des signes de FSHD avaient un profil normal avec des allèles de haut poids moléculaire $(>50 \mathrm{~kb})$ en simple digestion EcoRI, et un fragment délété à $19 \mathrm{~kb}$ et $13 \mathrm{~kb}$ respectivement après double digestion EcoRI/BlnI, suggérant un remaniement complexe de la région D4Z4 sur un chromosome 4. Chez un patient, les signes de FSHD étaient associés à la présence d'une autre maladie musculaire génétiquement prouvée, la dystrophie myotonique de type I ( 800 répétitions CTG dans le gène DM1). Discussion : Le diagnostic moléculaire de routine de la FSHD est complexe avec un taux d'échec élevé dans notre série, du à des problèmes technniques concernant la manipulation de grands fragments d'ADN. Le LGE reste la technique la plus commune pour le diagnostic de FSHD, suffisante en général pour des allèles de moins de $30 \mathrm{~kb}$ (7 UR). Le PFGE est indispensable pour séparer les allèles de grande taille, limite entre la zone normale et pathologique. De plus, lorsque un allèle délété est mis en évidence, il est difficile de déterminer le variant associé $\mathrm{qA} / \mathrm{qB}$. Les réarrangements complexes de D4Z4 suspectés par les profils atypiques en southern blot mis en évidence chez quelques patients, résultent probablement des échanges chromosomiques entre les régions $4 \mathrm{q}$ and $10 \mathrm{q}$. Avec les techniques de diagnostic classique, il est impossible de déterminer précisément la nature de ces réarrangements complexes de D4Z4. Les profils moléculaires atypiques pourraient apporter des éléments d'explication aux mécanismes physiopathologiques encore inconnus de la FSHD. Mais cela nécessite de nouveaux moyens d'exploration de la région $4 \mathrm{q} 35$, à la fois pour le diagnostic et la recherche fondamentale.

Mots-clés: dystrophie facio-scapulo-humérale, D4Z4, Southern-blot, PFGE.

\section{IP380 / 279. COMMD1 UNE PROTÉINE DE L'INFLAMMATION} ET DE L'ADRESSAGE LIÉ À CFTR

L. Drevillon (1, 2), G. Tanguy $(1,2)$, A. Hinzpeter $(1,2)$, N. Arous $(1,2)$, B. Saubamea (3), P. Fanen $(1,2)$

(1) INSERM U841 Département de Génétique, Institut Mondor de Recherche Biomédicale, Créteil ; (2) Université Paris 12, Créteil ; (3) EA 362-Service de Biologie Cellulaire-Service Commun d'Imagerie Cellulaire et Moléculaire Faculté de Pharmacie, Paris

Chez plus de $90 \%$ des patients, la mucoviscidose se caractérise par une absence quasi totale de la protéine mutée (F508del-CFTR) à la membrane apicale des épithéliums. Les malades présentent une symptomatologie variée, dominée par la gravité de l'atteinte pulmonaire et une réponse inflammatoire inadaptée. Afin d'identifier de nouvelles protéines qui interagissent avec la protéine CFTR durant son processus de maturation, nous avons utilisé la technique du criblage double-hybride de levure avec la troisième boucle cytoplasmique du CFTR comme proie. Nous avons ainsi mis en évidence COMMD1, une protéine impliquée dans le métabolisme du cuivre, comme un nouveau partenaire protéique de CFTR. Cette interaction a été tout d'abord vérifiée par des expériences de co-immunoprécipitation avec la totalité de la protéine CFTR (CFTR-wt ou F508del) exprimée de façon hétérologue dans les cellules HEK293 puis confirmée par la co-immunoprécipitation de CFTR et COMMD1 exprimés de façon endogène dans les cellules HT-29. La localisation sub-cellulaire de la protéine COMMD1 dans des cellules CF et non CF a montré une co-localisation partielle de CFTR avec COMMD1 dans le cytoplasme par des expériences d'immunocytochimie. La répartition de COMMD1 est cependant à la fois nucléaire et cytoplasmique où elle présente un aspect vésiculaire. Dans les cellules F508del, la forme nucléaire de COMMD1 est fortement augmentée par rapport aux cellules sauvages. Cette différence de localisation nucléaire et cytoplasmique dans les cellules CF et non CF a été confirmée par des expériences biochimiques de fractionnement nucléaires et cytoplasmiques. Par conséquent, l'expression de F508del-CFTR induit 
une relocalisation de COMMD1 dans le noyau, suggérant un rôle nucléaire de COMMD1. Par des expériences de biotinylation des protéines de surface nous avons montré que l'adressage à la membrane plasmique du canal CFTR-wt est diminué de moitié lorsque l'expression de la protéine COMMD1 est éteinte de $90 \%$ par ARN interférence. COMMD1 est donc impliquée dans l'adressage voire le recyclage du canal CFTR à la membrane apicale. La caractérisation plus précise de l'étape concernée est en cours d'évaluation à la fois par des méthodes biochimiques et d'immunocytochimie afin de déterminer le rôle de COMMD1 dans ce compartiment vésiculaire cytoplasmique. COMMD1 est le prototype d'une nouvelle famille de protéines inhibant NF-kappaB mais de nouvelles fonctions sont actuellement décrites qui permettent de cataloguer COMMD1 comme une protéine à effet pléiotrope au carrefour de nombreux processus inflammatoires et de régulation de la stabilité des protéines via la voie ubiquitineprotéasome. Nous avons montré que COMMD1 est un nouveau partenaire de la protéine CFTR, qu'il participe à son adressage membranaire et que l'expression de F508del-CFTR induit la redistribution COMMD1 dans le noyau. Nos perspectives visent à : 1) déterminer son rôle dans la réponse inflammatoire particulière à la mucoviscidose via les conséquences fonctionnelles de son expression nucléaire et 2 ) évaluer son rôle dans l'adressage d'autres canaux à la membrane des cellules épithéliales. Une connaissance approfondie de ces mécanismes contribuera à proposer des traitements novateurs pour lutter contre l'inflammation et/ou corriger le routage de CFTR chez les patients atteints de mucoviscidose. Ce travail fait l'objet d'un soutien par l'Association Vaincre la Mucoviscidose

Mots-clés : mucoviscidose, COMMD1, adressage.

- 381 / 281. APPLICATION DE LA CGH-ARRAY DÉDIÉE POUR LA MISE EN ÉVIDENCE DES GRANDS RÉARRANGEMENTS GÉNIQUES

E. Rouleau (1), S. Tozlu (1), C. Andrieu (1), C. Lefol (1), V. Bourdon (2), T. Nogushi (2), C. Nogues (1), I. Bieche (1), S. Olschwang (2), H. Sobol (2), R. Lidereau (1)

(I) Laboratoire d'oncogénétique, centre René-Huguenin, Saint-Cloud, France; (2) Département d'oncogénétique, Institut Paoli-Calmettes, Marseille, France

Introduction : Des grands réarrangements géniques sont observées dans de nombreux gènes (CFTR, DMD, BRCAI, FVIII, HPRT, BMPR2...). Nous avons utilisé notre expertise technique en $\mathrm{CGH}$-array haute résolution pour mettre en place une nouvelle approche dans la détection et la caractérisation rapide des grands réarrangements géniques. Matériel et méthode : Trois puces $11 \mathrm{k}$ de CGH-array Agilent ${ }^{\circ}$ dédiées ont été développées et analysées par le Centre René Huguenin pour les gènes BRCAI (1679 oligonucléotides), BRCA2 (1389), MLH1 (1031), MSH2 (789) et APC (1573). 10 échantillons BRCAI/BRCA2, et 3 échantillons $M L H 1 / M S H 2$ provenaient du Centre René Huguenin. 4 échantillons $A P C$ provenaient de l'Institut Paoli Calmettes. Un système standardisé d'analyse des puces a été développé au sein du Centre René Huguenin. Tous les échantillons ont été validés en MLPA. Résultats : Tous les grands réarrangements détectés par MLPA ont été confirmés par les puces dédiées. La technique de CGH-array a pu mettre en évidence des délétions de $500 \mathrm{pb}$ à plusieurs centaines de $\mathrm{kb}: A P C$ (4 délétions), BRCA1 (7 délétions), MSH2 (2 délétions). Les duplications sont clairement observés : $B R C A 1$ (2 duplications), BRCA2 (1 duplication), MLHI (1 duplication). Nous avons mis ainsi en évidence une délétion avec une insertion et une délétion de l'ensemble du gène $A P C$. La plupart des altérations ont pu être caractérisées par une simple PCR. La résolution de la CGH-array haute résolution a été de $1 \mathrm{~kb}$. Conclusion : L'application de la CGH-array dédiée pour les grands réarrangements géniques semble prometteuse, soit en première approche, soit en confirmation. Elle permet d'avoir une vue panoramique des gènes. La quantité d'information limite les faux-positifs. Elle permet d'étudier les altérations dans les grands exons. Enfin, il est possible de caractériser rapidement les remaniements (délétion ou duplication) par une simple PCR. L'utilisation de cette technique nécessite néamoins une sélection rigoureuse des oligonucléotides et un processus d'analyse dédié.

Mots-clés : CGH-array, grands réarrangements, points de rupture.

IP382 / 288. L'HYPERMÉTHYLATION DU GÈNE DU TISSUE FACTOR PATHWAY INHIBITOR-2 EST ASSOCIÉE À UNE DIMINUTION DE SON EXPRESSION DANS LA PLAQUE D'ATHÉROSCLEROSE CAROTIDIENNE

C. Zawadzki (1), N. Chatelain (1), A. Vincentelli (1), B. Quesnel (2), S. Susen (1), E. Jeanpierre (1), A. Bauters (1), S. Haulon (3), D. Corseaux (1), G. Torpier (4), B. Staels (4), E. Van Belle (1), B. Jude (1)

(1) INSERM ERI-9 et Université de Lille 2 EA-2693, Lille, France; (2) Service des Maladies du Sang, CHRU de Lille, France; (3) Clinique de
Chirurgie Cardiovasculaire, CHRU de Lille, France ; (4) INSERM U545, Institut Pasteur de Lille, France

Introduction : Le Tissue Factor Pathway Inhibitor-2 (TFPI-2) est un inhibiteur de sérine-protéase de type Kunitz exprimé dans la plaque d'athérosclérose par le macrophage, la cellule endothéliale ou la cellule musculaire lisse. Le TFPI- 2 y présenterait un effet stabilisant de la chape fibreuse superficielle en neutralisant l'activité des métalloprotéases matricielles (MMP) responsables de la dégradation de la matrice extracellulaire. Le promoteur du gène du TFPI-2 présente plusieurs ilôts CpG dont l'hyperméthylation est associée à une diminution de son expression. Cette régulation épigénétique de l'expression du TFPI-2 a été mise en évidence dans de nombreuses tumeurs cancéreuses mais n'a pas à ce jour été étudiée dans l'athérosclérose. L'objectif de ce travail a été d'étudier la méthylation du gène du TFPI-2 dans l'athérosclérose avec ses conséquences sur l'expression du transcrit TFPI-2. Le statut de méthylation du gène du TFPI-2 a également été confronté aux données clinico-biologiques des patients. Matériel et méthodes. La méthylation du gène du TFPI-2 a été étudiée par Methylation Specific PCR (MSP) dans l'ADN de 59 plaques d'athérosclérose carotidienne et de 26 artères mammaires contrôles. Trois systèmes de MSP différents ont été développés pour étudier 17 des 29 motifs CpG localisés sur un fragment de $268 \mathrm{pb}$ situé de part et d'autre du site d'initiation de la transcription (MSP1, 2 et 3 situés respectivement de l'extrémité 5' vers 3'). La bisulfitation de l'ADN a été réalisée par CpGenome DNA Modification kit ${ }^{\circ}$, Chemicon. Le transcrit TFPI-2 a été mesuré par PCR en temps réel (Taqman ABI Prism $7000^{\circ}$, Applied Biosystems). Résultats : Treize plaques ( $22 \%$ ) étaient méthylées avec MSP1, aucune avec MSP2 et 3 avec MSP3 (5\%). Aucune artère mammaire n'était retrouvée méthylée dans aucun des 3 systèmes. Ces résultats de MSP ont été validés par clonage et séquençage des ampligènes de MSP. Le niveau de transcrit TFPI-2 est significativement plus bas dans les plaques méthylées que dans les non méthylées et que dans les artères mammaires ( $\mathrm{p}=0,03$ et 0,009 respectivement). Plusieurs $\mathrm{CpG}$ étudiés par le système MSP1 sont localisés dans des motifs Spl et egr-1/Spl en -214 et -118 pb respectivement, impliqués dans l'inhibition d'expression du TFPI-2. La prévalence des facteurs de risque cardiovasculaire et des accidents ischémiques cérébraux n'était pas différente entre les plaques méthylées et non méthylées. Conclusion: Cette étude démontre pour la première fois que l'expression du TFPI-2 peut être régulée par hyperméthylation dans l'athérosclérose. Ce mécanisme de régulation épigénétique est mis en évidence dans environ un quart des plaques testées. L'hyperméthylation du gène du TFPI-2 y est associée à une diminution de l'expression de son transcrit. Son impact sur la régulation des MMP et sur la fragilité de la plaque d'athérosclérose reste à déterminer.

Mots-clés : TFPI-2, méthylation, athérosclérose.

aP383 / 289. ASSOCIATION GÉNÉTIQUE ENTRE DES POLYMORPHISMES DE CANAUX IONIQUES CARDIAQUES ET LA LONGUEUR DE L'INTERVALLE QTC CHEZ DES SUJETS SAINS L. Gouas (1, 2), V. Nicaud (3), S. Chaouch (1), M. Berthet (1), A. Forhan (4) A. Pfeufer (5), L. Tiret (3), B. Balkau (4), P. Guicheney (1) et le groupe d'Étude DESIR

(1) INSERM U582, GH Pitié-Salpêtrière, Université Paris 6, Paris, F-75013, France ; (2) Univ. Clermont I, UFR Médecine, CHU ClermontFerrand, Service de Cytogénétique Médicale, Clermont-Ferrand, F-63000, France ; (3) INSERM UMR S 525, GH Pitié-Salpêtrière-Université Paris 6, Paris, F-75634 France ; (4) INSERM U780, Villejuif, F-94807, France ; (5) Institute of Human Genetics, Munich, Allemagne

Introduction : L'intervalle QT, mesuré sur à l'électrocardiogramme et corrigé sur la fréquence cardiaque $(\mathrm{QTc})$, reflète la durée de la repolarisation ventriculaire cardiaque. La durée de l'intervalle QTc au repos est très variable d'un individu à un autre dans la population (340-440 ms), mais relativement stable chez un même sujet et sous le contrôle de facteurs génétiques comme cela a été montré par des études de jumeaux mono- et dizygotes. Nous avons réalisé des études d'association génétique entre des polymorphismes de gènes codant des sous-unités de canaux ioniques cardiaques potassiques (KCNQ1, KCNE1, KCNH2, KCNE2) et sodique (SCN5A), une protéine régulatrice de la NO-synthase neuronale (NOSIAP), et la durée de l'intervalle QTc. Méthode: Vingt-quatre polymorphismes (11 KCNQ1, 4 KCNE1, 2 KCNH2, 1 KCNE2, 5 SCN5A, 1 NOS1AP) ont été génotypés par F.R.E.T (LightCycler) ou par séquençage dans deux groupes de 200 sujets présentant les intervalles QTc les plus courts (333-365 ms) et les plus longs (394-432 ms) d'une cohorte de 2008 assurés sociaux sains participant à l'étude prospective DESIR (Données Epidémiologiques sur le Syndrome de l'Insulino-Résistance). Résultats : Tous les polymorphismes étudiés étaient en équilibre de Hardy-Weinberg dans les deux groupes, excepté rs 10494366 (NOSIAP) pour lequel on observe 
un excès d'homozygotes GG (allèle mineur) dans le groupe de sujets présentant les QTc longs $(p=0,01)$. Les allèles mineurs rs $1805123 \mathrm{C}$ de $K C N H 2$, et rs1805126 T et IVS24+116 A de SCN5A étaient significativement plus fréquents dans le groupe de sujets présentant les intervalles QTc courts $(\mathrm{OR}[95 \% \mathrm{CI}]=0,64(0,46-0,88), \mathrm{p}=0,0055 ; 0,65(0,48-0,89)$, $\mathrm{p}=0,0063 ; 0,61(0,40-0,92) ; \mathrm{p}=0,018$, respectivement $)$. En revanche, les allèles mineurs rs757092 $\mathrm{G}$ de $K C N Q 1,253 \mathrm{~A}$ de $K C N E 1, \mathrm{rs} 3815459$ A de $K C N H 2$, et rs $1805124 \mathrm{G}$ et IVS9-3 A de SCN5A, et rs10494366 G de NOS1AP étaient significativement plus fréquents dans le groupe de sujets présentant les intervalles QTc longs $(\mathrm{OR}[95 \% \mathrm{CI}]=1,48(1,10-2,01)$, $\mathrm{p}=0,011 ; 4,21(1,17-15,16), \mathrm{p}=0,028 ; 1,48(1,05-2,10) \mathrm{p}=0,026 ; 1,52$ $(1,11-2,10), p=0,010 ; 1,66(1,18-2,35), p=0,0039 ; 1,72(1,28-2,32)$, $\mathrm{p}=0,0004)$ ). Par ailleurs, une interaction entre les polymorphismes rs 1805123 de $K C N H 2$ et $2031+932 \mathrm{~A}>\mathrm{G}$ de $K C N Q 1$ a été mise en évidence. Conclusion: Notre étude montre ou confirme l'existence d'une association génétique de plusieurs polymorphismes de canaux ioniques cardiaques (KCNQ1,KCNE1,KCNH2, SCN5A) et de NOSAPI avec la durée de la repolarisation ventriculaire chez des sujets sains d'origine caucasienne. Ces associations, parfois validées sur de plus larges cohortes, suggèrent que ces polymorphismes pourraient moduler le risque d'arythmies chez des patients atteints d'une pathologie primaire de la repolarisation ventriculaire cardiaque ou d'une cardiomyopathie.

Mots-clés : intervalle QTc, polymorphismes, canaux ioniques cardiaques.

\section{$\square$ P384 / 291. CINQ NOUVELLES MUTATIONS DANS LE GÈNE EFNB1 RESPONSABLES DE DYSPLASIE CRÂNIO-FRONTO-NASALE}

C. Collet (1), V. des Portes (2), P. Edery (3), H. Gaspard (4), M. GerardBlanluet (5), M. Holder (6), J.-M. Launay (1), M. Lenne (1), S. Marlin (7), G. Morin (8), G. Pierquin (9), M. Rajguru (10), D. Renier (11), J.-L. Laplanche (1)

(1) Service de Biochimie et Biologie Moléculaire, Hôpital Lariboisière, Paris, France ; (2) Service de Neuropédiatrie, Hôpital Debrousse, Lyon, France; (3) Service de Génétique, Hôpital Debrousse, Lyon, France ; (4) Institute of Medical Genetics, Schwerzenbach, Switzerland; (5) Service de Génétique Clinique, Hôpital Robert-Debré, Paris, France; (6) Service de Génétique Clinique, Hôpital Jeanne-de-Flandre, Lille, France ; (7) Service de Génétique Clinique, Hôpital Trousseau, Paris, France ; (8) Département de Pédiatrie, Génétique Clinique, Hôpital Nord, Amiens, France: (9) Service de Génétique, CHU de Liège, Belgique: (10) Service de Gynécologie-Obstétrique, Hôpital Bichat-Claude Bernard, Paris, France ; (11) Service de Neurochirurgie pédiatrique, Hôpital Necker, Paris, France

Introduction : La dysplasie crânio-fronto-nasale (DCFN, MIM304110) est une maladie rare liée à 1 'X, qui montre de façon paradoxale une expression plus sévère chez les filles à l'état hétérozygote que chez les garçons à l'état hémizygote. Les signes cliniques majeurs retrouvés chez les filles sont un hypertélorisme, une asymétrie craniofaciale, une craniosténose coronale (inconstante) soit uni- ou bilatérale, un nez bifide, une arête nasale large, des cheveux secs, ondulés et crépus, une implantation basse sur la nuque, des ongles striés longitudinalement, un gros orteil large et un espace entre $1^{\text {er }}$ et $2^{\mathrm{e}}$ orteil et une dysplasie thoracique. La plupart des garçons sont légèrement affectés (hypertélorisme seulement). Cette affection survient chez moins d'un nouveau-né sur 100000 et ce syndrome peut être dû à des mutations du gène $E F N B 1$. Ce gène, composé de 5 exons est localisé en Xq3.1., code pour un des membres de la famille ephrin. La protéine ephrin-BI est une protéine transmembranaire de type I et un ligand pour le récepteur EphB1 tyrosine kinase qui est un des régulateurs de la morphogénèse des tissus embryonnaires, caractérisé par une région intracellulaire contenant de multiples résidus tyrosine et un PDZ domaine (B1). Après un contact cellule-cellule le récepteur EphB1 interagit avec le ligand éphrine-B1. Actuellement une cinquantaine de mutations ont été décrites dans les 5 exons du gène $E F N B 1$ avec une majorité des mutations retrouvées dans les exons 2 et 3 codants pour le domaine extracellulaire de la protéine. Nous avons analysé les 5 exons du gène EFNBI chez 9 patients atteints de DCFN et nous avons identifié 6 mutations dont 5 nouvelles. Méthodes : 9 prélèvements d'ADN de patients âgés de 1 mois à 45 ans atteints d'une DCFN ( 7 filles et 2 garçons) ont été analysés par séquençage direct bidirectionnel. Résultats : 6 mutations sur les 9 patients atteints d'une DCFN ( 3 formes familiales et 6 sporadiques) ont été retrouvées dans le gène EFNBI (c.129-2A>G, p.R66PfsX8, p.L80R, p.C89R, p.N120KfsX11 et p.K345RfsX46). En dehors de la mutation p.C89R, elles sont toutes non décrites. Les mutations sont toutes situées dans l'exon 2 à l'exception de p.K345RfsX46 (exon 5). Discussion : Les mutations amènent majoritairement à un décalage de lecture, voire à une modification de l'épissage et elles sont donc responsables de l'apparition d'un codon stop prématuré. prédominance des mutations privées que ce soit dans les formes familiales et sporadiques. Dans la littérature, une mutation dans le gène EFNBI est retrouvée dans environ $90 \%$ des cas. Les non réponses sont dues : soit à la présence de grandes délétions non détectables par séquençage (en cours d'exploration), soit à des mutations dans des régions régulatrices du gène $E F N B 1$ ou encore à un mosaïcisme.

Mots-clés : dysplasie crânio-fronto-nasale, mutation, EFNB1.

\section{PP385 / 292. BBS12, UNE NOUVELLE PROTÉINE VERTÉBRÉE SPÉCIFIQUE IMPLIQUÉE DANS LE SYNDROME DE BARDET-BIEDL}

C. Stoetzel (1), J. Muller (2), V. Laurier (1), E. Davis (3), P. Sarda (4), C. Hamel (5), T. de Ravel (6), C. Thibault (2), J.M. Danse (1), V. Marion (1), S. Héllé (1), A. Verloes (7), D. Bonneau (8), N. Katsanis (3), O. Poch (2), J.L. Mandel $(2,9)$, H. Dollfus $(1,10)$

(I) Laboratoire de Génétique Médicale EA3949, Université Louis-Pasteur, Faculté de Médecine, Strasbourg ; (2) Institut de Génétique et de Biologie Moléculaire et Cellulaire, INSERM, U596, CNRS, UMR7104, Illkirch, Strasbourg; (3) McKusick-Nathans Institute John Hopkins University, Baltimore, Maryland, USA ; (4) Service de Génétique Médicale, CHU Arnaud-de-Villeneuve, Montpellier ; (5) Institut des Neurosciences, CHU Saint-Éloi, Montpellier ; (6) Centre of Human Genetics, University Hospital Gasthuisberg Belgique; (7) Unité de Génétique Clinique, Hôpital Robert-Debré Paris ; (8) Service de Génétique, CHU d'Angers; (9) Collège de France, chaire de génétique humaine, Illkirch Strasbourg; (10) Service de Génétique Médicale et Centre de Référence pour les Affections Génétiques Ophtalmologiques, CHU, Strasbourg

Introduction: Le syndrome de Bardet-Biedl (BBS) (OMIM 209900) est une maladie autosomique récessive caractérisée principalement par : une rétinopathie pigmentaire précoce, une obésité, une polydactylie, un hypogonadisme, un déficit cognitif variable et des anomalies uro-néphrologiques. À ce jour, douze gènes (BBS1 à 12) ont été identifiés. Nous rapportons ici l'identification du gène $B B S 12$. Méthode: La stratégie d'identification a combiné une approche par cartographie par homozygotie utilisant les puces SNP 10K (Affymétrix) et une approche in silico de génomique comparative et des outils de modélisation moléculaire. Cette approche a été complétée par des tests fonctionnels sur le poisson zèbre inhibant l'expression du gène d'intérêt à l'aide d'oligonucléotides de type morpholino spécifiques. Résultats : L'analyse de l'ADN de deux familles gitanes en puces $10 \mathrm{~K}$ Affymétrix a montré une région d'homozygotie, haplo-identique de $6 \mathrm{Mb}$ sur le chromosome $4 \mathrm{q} 27$. Un codon stop homozygote dans le gène $F L J 35630$ a été identifié pour les patients des deux familles. L'analyse du reste de notre cohorte a révélé que au moins $5 \%$ des patients BBS sont porteurs de mutations dans ce gène. Ce gène dénommé $B B S 12$ code pour une protéine vertébrée spécifique de type chaperonine-like. Les tests fonctionnels dans le poisson-zèbre par inactivation spécifique de $B B S 12$ ont démontré des défauts dosage-dépendant des processus précoces des mouvements de gastrulation similaires aux phénotypes d'inhibition des autres gènes BBS. Discussion : Le gène BBS12 tout comme les gènes $B B S 10$ et 6 code une protéine ressemblant aux chaperonines de type II. Elles pourraient faire partie d'un complexe de type chaperonne qui serait impliqué dans des fonctions ciliaires. Corroborant cette hypothèse, les autres protéines BBS, notamment les BBS1, 2, 4, 5, 7, 8 et 9 forment un complexe multi protéique ne comportant pas les trois protéines chaperonnes. La caractérisation du rôle de BBS12 avec les BBS10 et le BBS6 au niveau cellulaire devrait nous permettre de disséquer la physiopathologie du complexe ciliaire dans ce syndrome

Mots-clés : ciliopathie, syndrome de Bardet Biedl, identification BBS12.

\section{IP386 / 297. BASES MOLÉCULAIRES ET CLINIQUES DU SYN DROME DE PITT-HOPKINS, UNE ENCÉPHALOPATHIE SYN- DROMIQUE SÉVÊRE}

L. De Pontual (1), M. Rio (2), Y. Mathieu (1), N. Boddaert (3), C. Soufflet (4), P. Plouin (4), C. Picard (5), A. Durandy (5), S. Lyonnet (1, 2), A Munnich $(1,2)$, L. Colleaux (2), J. Amiel $(1,2)$

(1) INSERM U-781; (2) Département de génétique ; (3) Service de radiologie pédiatrique et INSERM U-797; (4) Service de neurophysiologie et INSERM U-663; (5) INSERM U-429, Université Paris-Descartes, Faculté de Médecine : AP-HP, Hôpital Necker-Enfant Malades, Paris, France

Le syndrome de Pitt-Hopkins (PHS) se caractérise par un retard psychomoteur sévère, une épilepsie et des accès d'hyperventilation ayant permis d'individualiser ce syndrome en 1978. Il s'y associe un retard de croissance et une microcéphalie post-natale, une dysmorphie faciale reconnaissable, ainsi que des anomalies à l'EEG et à l'IRM cérébrale. Tous les cas rapportés étant sporadiques, nous avons recherché un micro remaniement 
chromosomique par hybridation génomique comparative pan génome chez 4 patients supposés atteints de PHS et identifié une délétion de novo de $1,8 \mathrm{Mb}$ en $18 \mathrm{q} 21.1$ chez une patiente. La délétion incluait 4 gènes dont $T C F 4$, un facteur de transcription à motif basic HLH de classe I. Le séquençage direct a permis d'identifier une mutation héterozygote de novo de TCF4 chez 5 patients. Le mécanisme le plus probable est une perte de fonction par haplo-insuffisance mais un effet dominant-négatif reste possible pour les mutations faux sens du domaine basique. L'étude de l'expression de TCF4 au cours du développement embryonnaire humain révèle une expression ubiquitaire mais avec des niveaux d'expression extrêmement variables. Une étude plus fine du phénotype clinique, immunologique, de l'imagerie cérébrale et de l'EEG des patients atteints de PHS est en cours et sera détaillée. Tcf 4 participe à l'ontogénie des précurseurs lymphoïdes B et les souris $T c f 4 \%$ - présentent une diminution de ces précurseurs de l'ordre de $50 \%$. Ces souris meurent dans les premiers jours de vie sans cause identifiée, le déficit immunitaire ne pouvant être responsable d'une mortalité si précoce. Nous avons entrepris l'étude de ces souris par pléthysmographie et électrophysiologie.

Mots-clés : syndrome de Pitt-Hopkins, encéphalopathie, TCF4.

\section{EP387 / 299. QU'EST CE QUE LE SYNDROME COFS ? ÉTUDE CLINIQUE ET MOLÉCULAIRE DE TROIS NOUVEAUX CAS ET REVUE DE LA LITTÉRATURE}

V. Laugel (1), C. Dalloz (1), M. Durand (1), E. Tobias (2), J. Tolmie (2), D. Martin-Coignard (3), V. Valayannopolous (4), V. Drouin-Garraud (5), H. Dollfus (1)

(1) Laboratoire de Génétique Médicale, EA 3949, HUS, Strasbourg ; (2) Génétique Médicale, Glasgow, UK; (3) Service de Génétique Médicale, CHU du Mans ; (4) Service de Neuropédiatrie, Hôpital Necker, Paris ; (5) Génétique Médicale, $\mathrm{CHU}$ de Rouen

Le syndrome cérébro-oculo-facio-squelettique (COFS) [MIM214150], initialement identifié dans une population d'Indiens du Manitoba, est une maladie génétique autosomique récessive caractérisée par l'association microcéphalie, cataracte, microphtalmie, arthrogrypose et retard de croissance. Certaines de ces caractéristiques rapprochent le syndrome COFS du syndrome de Cockayne [MIM133540-216400] et les deux entités partagent le même défaut biochimique spécifique dans une voie de réparation de l'ADN, la voie TCR (« transcription-coupled repair »). Les patients de la description originale du syndrome COFS portent une même mutation homozygote dans le gène majeur du Cockayne (CSB), mais seulement cinq autres cas de COFS ont été étudiés sur le plan moléculaire en dehors de cet isolat. De nombreuses descriptions cliniques n'ont pas été confirmées sur le plan moléculaire et entretiennent la confusion sur la délimitation du cadre nosologique. Nous présentons les données cliniques et moléculaires de trois nouveaux patients COFS associés à des mutations dans le gène $C S B$. Les trois patients présentent les signes cardinaux du syndrome avec microcéphalie, cataracte congénitale, microphtalmie et arthrogrypose. Ils présentent aussi un retard de croissance essentiellement postnatal, un retard psychomoteur sévère, des difficultés alimentaires néonatales, une hypotonie axiale ainsi qu'une dysmorphie particulière distincte de celle du syndrome de Cockayne. Les fibroblastes issus de ces trois patients présentent une sensibilité particulière aux rayons ultraviolets et le défaut classique de réparation dans la voie TCR. L'étude moléculaire a mis en évidence des mutations dans le gène CSB. En fonction de ces données, nous proposons des critères cliniques de définition du syndrome COFS et nous discutons des liens avec le syndrome de Cockayne et avec d'autres syndromes apparentés.

Mots-clés : COFS, cockayne, CSB.

\section{nP388 / 300. DIAGNOSTIC MOLÉCULAIRE DES ÉPILEPSIES} MYOCLONIQUES PROGRESSIVES (EPM1 ET 2)

C.A. Bourdin (1), N. Boutry (1), P. Ryvlin (2, 3), F. Mauguière (3), P. Genton (4), A. Malafosse (5), A. Calender (1), G. Lesca $(1,2)$

(1) Laboratoire de Génétique, Hôpital Édouard-Herriot, Lyon; (2) CTRSIdée, Lyon; (3) Service d'Épileptologie, Hôpital Neurologique, Bron; (4) Centre Saint-Paul, Marseille ; (5) Division de Neuropsychiatrie, Hôpital Belle-Idée, Genève

Les maladies de Lafora (EPM2) et d'Unverricht-Lundborg (EPM1) font parties des épilepsies myocloniques progressives dont la triade clinique comprend des myoclonies, une épilepsie généralisée de type tonico-clonique ou absence et une atteinte dégénérative du système nerveux central avec démence et ataxie de gravité variable. Elles sont transmises selon un mode autosomique récessif. La maladie de Lafora représente une forme fréquente et particulièrement grave d'EPM. L'âge de début se situe entre 6 et 19 ans et son évolution conduit à une démence en quelques années et dans la plupart des cas, au décès du patient après 2 à 10 ans. Elle est causée par des mutations des gènes EPM $2 A$ et $N H L R C 1 / E P M 2 B$. A l'inverse, la maladie d'Unverricht-Lundborg débute entre 6 et 18 ans et son évolution se fait souvent vers une stabilisation des symptômes à l'âge adulte sans déficit cognitif progressif. Elle est très majoritairement liée à une expansion d'un dodécamère au niveau du promoteur du gène CSTB, bien que plusieurs mutations ponctuelles aient été également décrites. Nous avons mis en place le diagnostic moléculaire des EPM au CHU de Lyon, en collaboration avec le Pr. Alain Malafosse, pour répondre à la demande de la communauté des neurologues et des épileptologues. Le gène CSTB est étudié par Southern Blot pour détecter l'expansion du dodécamère puis par séquençage pour rechercher les mutations ponctuelles. L'étude des gènes EPM $2 A$ et $N H L R C 1 / E P M 2 B$ est effectuée par séquençage, éventuellement complétée par l'étude de l'ADNc à la recherche de délétions des exons 2 ou 3. Dans un premier temps, nous avons complété les analyses de patients français débutées à Genève en séquençant la séquence codante du gène CSTB chez 98 patients et en effectuant également le Southern Blot chez 28 d'entre eux. Nous avons ensuite étudié une série de deux groupes de 22 et 20 cas index présentant, respectivement, un tableau évocateur de maladie d'Unverricht-Lundborg ou de maladie de Lafora. Pour le gène CSTB, nous avons confirmé le diagnostic chez 8 patients dont 6 homozygotes pour l'expansion du dodécamère et 2 hétérozygotes pour cette expansion et porteurs d'une mutation du site accepteur d'épissage de l'exon 2 (c.67-1G>C), sur l'autre allèle. Nous avons confirmé le diagnostic de maladie de Lafora chez 6 patients. Cinq d'entre eux sont porteurs de mutations du gène $E P M P 2 A$, dont 2 porteurs d'une mutation non-sens à l'état homozygote (c. $259 \mathrm{~A}>\mathrm{T}$ et c. $834 \mathrm{C}>\mathrm{A}), 2$ porteurs d'une mutation non-sens et d'une délétion intra-exonique (c.721 C > T + c.102_133del32) ou une impliquant l'exon 2 dans sa totalité (c.721 C > T + c.302- ?_476+ ?del) et un patient dont les mutations sont en cours de caractérisation. Un autre patient est hétérozygote composite pour des mutations du gène NHLRC1/EPM2B (c.940_943dupCTCT+c.1163_1164delAA). Chez un patient présentant des lésions histologiques typiques de la maladie de Lafora, nous n'avons pas mis en évidence de mutation. L'identification des mutations en biologie moléculaire est importante pour la confirmation du diagnostic et le conseil génétique mais également pour le pronostic et l'orientation thérapeutique des EPM, certains traitements pouvant avoir un effet délétère. L'absence de mutation ne remet pas forcément en doute le diagnostic de maladie de Lafora ou d'Unverricht-Lundborg, d'autres gènes étant vraisemblablement impliqués dans ces maladies.

Mots-clés : épilepsie myoclonique progressive, diagnostic, mutations.

\section{EP389 / 315. DÉLÉTION DANS LE DOMAINE 5' UTR DU GÈNE CSB}

C. Dalloz (1), V. Laugel (1), M. Durand (1), A. Stary (2), V. Cormier-Daire (3), I. Desguerre (4), M. Renouil (5), J.M. Egly (6), A. Sarasin (2), H. Dollfus (1)

(1) Laboratoire de Génétique Médicale, EA 3949, HUS, Strasbourg ; (2) FRE 2939, IGR, Villejuif; (3) Département de Génétique Médicale, Hôpital Necker, Paris ; (4) Service de Neuropédiatrie, Hôpital Necker, Paris; (5) Service de Pédiatrie, Hôpital St-Pierre, La Réunion; (6) IGBMC, Illkirch

Situation du sujet : Le syndrome de Cockayne (CS) est une maladie génétique rare de transmission autosomique récessive, caractérisée par un retard de croissance statural associé à une maigreur extrême et progressive. Le tableau clinique comporte également une microcéphalie, un retard mental profond, une rétinopathie, une surdité neuro-sensorielle et une photosensibilité. Les enfants atteints décèdent en moyenne vers l'âge de 12 ans mais il existe une certaine variabilité à l'intérieur de ce syndrome avec des formes cliniques plus ou moins sévères. Les fibroblastes des patients CS présentent une anomalie dans une voie de réparation des lésions de l'ADN induites par les UV (ou voie TCR mis pour « transcription-coupled repair »), associée à des mutations des gènes $C S A$ et $C S B$. Il a été proposé qu'il pourrait exister une corrélation inverse entre la gravité du tableau clinique et la quantité résiduelle de protéine CSB. Nous rapportons ici les données cliniques et moléculaires de deux cas en contradiction avec ce modèle. Approches expérimentales et résultats : Les examens cliniques des deux patients révèlent un retard de croissance sévère, une microcéphalie, un retard mental, une cataracte congénitale et une dégénérescence rétinienne pigmentaire conduisant à un décès prématuré ( 6 et 8 ans). Les expériences d'irradiation aux UV des fibroblastes montrent une anomalie de la voie de réparation de l'ADN couplée à la transcription, caractéristique du phénotype CS. L'analyse par western-blot et par RT-PCR montre l'absence totale de la protéine et du messager de CSB dans les extraits fibroblastiques issus des patients. Le séquençage de l'ADN met en évidence une délétion génomique impliquant le domaine 5'UTR du gène CSB. 
Discussion : Ces patients sont les premiers cas CS décrits présentant un spectre clinique sévère associé à une absence complète du messager et de la protéine CSB suite à une mutation située en dehors de la partie codante du gène. Le seul autre cas connu d'absence complète de la protéine CSB présentait un tableau clinique particulièrement peu sévère. La comparaison de ces études nous permet de remettre en cause l'hypothèse avancée de la corrélation inverse génotype-phénotype et suggère que d'autres voies restent à explorer afin de comprendre les mécanismes moléculaires et cellulaires conduisant à la variété des phénotypes CS observés.

Mots-clés : syndrome de Cockayne, CSB, réparation de l'ADN.

EP390 / 317. IDENTIFICATION D'UNE MUTATION FAUX-SENS DANS LE GÈNE WFS1 IMPLIQUÉE DANS UN DIABĖTE AUTOSOMIQUE DOMINANT ASSOCIÉ À UNE SURDITÉ

S. Bannwarth (1), R. Valéro* (2), A. Figueroa (1), S. Roman (3), B. Vialettes (2) J.-C. Lambert (1), V. Paquis-Flucklinger (1), * Contribution égale (1) Service de Génétique Médicale, Hôpital Archet 2, CHU Nice: (2) Sevice de Nutrition, Maladies métaboliques, Endocrinologie, Hôpital La Timone, CHU Marseille ; (3) Service d'Oto-Rhino-Laryngologie, Hôpital La Timone, CHU Marseille

Introduction : Des mutations dans le gène WFSI sont responsables du syndrome de Wolfram (WS) ou DIDMOAD (Diabetes Insipidus, Diabetes Mellitus, Optic Atrophy and Deafness) (MIM\#222300). Il s'agit d'une affection rare, de transmission autosomique récessive qui se manifeste par l'association d'un diabète juvénile (DM) et d'une atrophie optique (AO). Les patients peuvent également développer un diabète insipide, une surdité neurosensorielle, des troubles urinaires, une atteinte neurologique et des désordres psychiatriques. Le gène WFSI comporte 8 exons et code pour une protéine transmembranaire appelée wolframine, dont le rôle reste à déterminer. Plus d'une centaine de mutations ont déjà été identifiées. La plupart sont dites « inactivatrices " (non sens ou avec décalage du cadre de lecture). Des mutations dans le gène WFS1 sont également responsables de maladies autosomiques dominantes telles que des surdités neurosensorielles sur les basses fréquences (LFSNHI : Low Frequency SensoriNeural Hearing Impairment). Les mutations responsables de LFSNHI different de celles observées dans le DIDMOAD. Ce sont des mutations faux sens, localisées dans la région C-terminale de la wolframine. Récemment, une équipe danoise a montré que la mutation c.2590G > A (E864K) dans WFS1 est responsable d'une association atrophie optique/surdité, de transmission autosomique dominante, dans une famille. Résultats : Nous avons retrouvé la mutation c.2590G >A (E864K) dans le gène WFS1 chez un patient, âgé de 60 ans, qui est atteint de diabète et de surdité, sans atrophie optique. Cette mutation a également été identifiée chez sa mère qui présente un diabète non-insulino dépendant, une surdité et une atrophie optique. Elle est absente chez les sujets non atteints de cette famille. Dans cette étude, nous avons également identifié un nouveau variant (R2280), présent chez le cas index mais absent chez sa mère. Le rôle de ce nouveau variant n'est pas connu et on ne peut exclure qu'il ait un rôle préventif dans le développement de l'atrophie optique. Conclusion: Cette étude démontre l'implication du gène WFS1 dans une nouvelle forme de diabète monogénique de transmission autosomique dominante, pouvant être associé à une surdité neurosensorielle et une atrophie optique. Il est donc fortement recommandé d'étudier systématiquement le gène WFSI chez des patients présentant un diabète associé à une surdité et/ou une atrophie optique, surtout en présence d'antécédents familiaux.

Mots-clés : WFSI, diabète, surdité.

\section{aP391 / 324. CARACTÉRISATION MOLÉCULAIRE DES TUMEURS DE PHÉNOTYPE BASAL-LIKE DE BAS GRADE HISTOPRONOSTIQUE}

M. Kossaï (1, 2, 5), L. Da Silva MD (1, 2), L. Reid (1, 2), P. Simpson (1, 2), P. Keith $(1,2)$, C. Clarke $(1,2)$, S. Manu $(1,2)$, A. Cayre $(5,6)$, S.R. Lakhani $(1,2,3)$, F. Penault-Llorca $(4,5,6)$

(1) Pathologie moléculaire et cellulaire,Université de Queensland, Brisbane, Australie; (2) The Queensland Institute of Medical Research, Brisbane, Australie; (3) The Royal Brisbane \& Women's Hospital, Brisbane, Australie ; (4) Université Clermont 1, Clermont-Ferrand ; (5) Centre JeanPerrin, Département de Pathologie, Clermont-Ferrand; (6) INSERM UMR 484, Clermont-Ferrand

Récemment, la découverte de profils moléculaires spécifiques a révolutionné l'approche diagnostique et thérapeutique du cancer du sein. Nous nous sommes intéressés à l'une des catégories agressives récemment démembrée, les tumeurs basal-like ( $15 \%$ des tumeurs mammaires). $\mathrm{Ce}$ n'exprimant ni les récepteurs hormonaux (récepteur aux œstrogènes RE. récepteur à la progestérone RP), ni l'oncogène HER-2. Ce sont des tumeurs pour lesquelles les ressources thérapeutiques actuelles sont limitées. Si la plupart de ces tumeurs sont de phénotype agressif (haut grade nucléaire, nécrose), certaines tumeurs présentent un phénotype de bas grade, moins agressif. Nous avons voulu évaluer si cela correspondait sur le plan moléculaire à un profil particulier. Le premier temps consistait à la collection des prélèvements, à la microdissection des zones d'intérêt et à la préparation des acides nucléiques à partir de coupes en paraffine. Ensuite, des analyses des profils d'expression des gènes par la technologie DASL, géniques par hybridation génomique comparative et protéiques par immunohistochimie ont été réalisées. Nos résultats préliminaires sont en faveur de profils d'expression différentiels pour les tumeurs basales de bas grade qui les rapprocheraient des tumeurs de bas grade mais non basal-like (REpositives). Ces travaux semblent montrer que le grade histopronostique est primordial dans la classification des tumeurs mammaires et dans le pronostic et qu'il serait plus déterminant que le statut hormonal. Ces travaux sont à confirmer sur une cohorte plus importante avant de poursuivre par des études fonctionnelles in vitro et in vivo.

Mots-clés : cancer du sein, basal-like de bas grade, analyse moléculaire.

EP392 / 328. RETARD MENTAL AVEC HYPOPLASIE CÉRÉBELLEUSE : DESCRIPTION DE 6 NOUVEAUX CAS PRÉSENTANT DES MUTATIONS DU GENE $O P H N I$

C. Lacoste (1), D. Bonneau (2), D. Lacombe (3), J.-M. Pedespan (4), N. Philip (5), N. Levy (1), C. Badens (1)

(1) Laboratoire de Génétique Moléculaire, $\mathrm{CHU}$ de la Timone, Marseille : (2) Service de Génétique, CHU d'Angers ; (3) Service de Génétique Médicale, CHU Pellegrin, Bordeaux; (4) Service de Neuropédiatrie, CHU Pellegrin, Bordeaux; (5) Service de Génétique Clinique, CHU de la Timone, Marseille

Le gène OPHN1 (Oligophrénine 1) localisé en Xq12, code pour une protéine comportant un domaine Rho-GAP et exprimée dans le système nerveux. Des mutations de ce gène entraînent un retard mental modéré à sévère, des anomalies cérébelleuses, une dysmorphie faciale discrète, un strabisme et une épilepsie précoce. Toutes les mutations décrites à ce jour sont des mutations non-sens ou entrainant un décalage du cadre de lecture ou des anomalies d'épissage. Les femmes porteuses hétérozygotes ne présentent pas de biais d'inactivation du chromosome $\mathrm{X}$ et peuvent être symptomatiques. Le diagnostic de mutations dans le gène $O P H N I$ est réalisé par criblage des 24 exons codant et des jonctions exon-intron par HPLC en conditions dénaturantes et séquençage des profils anormaux. Sur 56 cas index explorés récemment dans notre laboratoire, 3 patients présentaient une mutation pathogène dans $O P H N 1$ : une insertion d'un nucléotide (c.1738 dupC; p.Arg580Pro fs X30), une délétion de 11 nucléotides (c.1581 del(ACTTAACACTG) fs) et une mutation faux-sens située sur l'ATG (c.1 A>T ; p.Met1Leu). Dans la famille de ce dernier patient, un frère et un cousin germain étaient également atteints et porteurs de cette mutation. Chez un sixième patient, une mutation faux sens a été identifiée dans l'exon 2, p.A45T, mutation non rapportée dans les bases de données ni retrouvée dans une cohorte de 80 individus témoins, mais dont le caractère pathogène reste à démontrer. $\mathrm{Ce}$ patient présente un retard mental léger, une épilepsie partielle peu sévère, une dysmorphie discrète et une hypoplasie du vermis. Plusieurs polymorphismes ont également été identifiés dont le plus fréquent est p.Val39Ile que nous avons retrouvé dans $6,3 \%$ de la population générale et $10 \%$ de nos patients. Le phénotype des 5 patients présentant les mutations à fort potentiel pathogène est homogène et caractéristique de ce qui a été rapporté précédemment dans la littérature, notamment avec une hypoplasie vermienne, un retard global des acquisitions et une dysmorphie faciale caractéristique. Un strabisme est rapporté dans 2 cas et une épilepsie dans 3 cas sur 5 . Des analyses complémentaires du patient présentant la mutation faux sens A45T et de sa famille permettront peut être d'élargir le phénotype lié à des mutations d'OPHN1 à des formes moins sévères d'hypoplasie cérébelleuse.

Mots-clés : retard mental, oligophrénine, hypoplasie cervelet.

IP393 / 331. NOUVELLES MUTATIONS DANS LE GÈNE CLDN19 CHEZ TROIS FAMILLES AVEC HYPOMAGNÉSÉMIE FAMILIALE AVEC HYPERCALCIURIE ET NÉPHROCALCINOSE

A. Remenieras (1) ; A. Blanchard (2), N. Le Pottier (1), M. Godin (3), P. Houillier (2), X. Jeunemaître (1), R. Vargas-Poussou (1)

(I) Département de Génétique, Hôpital Européen Georges-Pompidou, Paris; (2) Département de Physiologie, Hôpital Européen Georges-Pompidou, Paris; (3) Service de Néphrologie, Hôpital de Bois-Guillaume, Rouen

M/S hors série $n^{\circ} 1$, vol. 24, janvier 2008 
Le syndrome hypomagnésémie familiale avec hypercalciurie et néphrocalcinose (FHHNC OMIM 248250) est une tubulopathie héréditaire à transmission récessive, caractérise par une fuite rénale de calcium et magnésium. Les manifestations cliniques constantes sont l'hypomagnésémie, l'hypercalciurie, la néphrocalcinose et l'évolution vers l'insuffisance rénale mais il peut exister également des lithiases, un retard de croissance, une polyurie, des infections urinaires, une acidose tubulaire et des atteintes oculaires. Cette maladie est due à des mutations du gène de la Claudine 16 (CLDN 16) (OMIM 603959). La claudine 16 est localisée dans les jonctions serrées des cellules de la branche large ascendante de l'anse de Henle (BLAH) où elle assure le passage paracellulaire de cations divalents. La perte de fonction de cette protéine est responsable d'un défaut de réabsorption de calcium et magnésium. Récemment, le gène de la Claudine 19 (CLDN 19) (OMIM 610036), a été impliqué chez des patients ayant le même phénotype rénal mais avec des atteintes oculaires constantes et sévères (myopie, nystagmus, colobome maculaire). La Claudine 19 est exprimée dans les jonctions serrées des cellules de la BLAH et de l'épithélium pigmenté de la rétine. Nous avons analysé le gène de la CLDN 19 dans neuf familles avec un tableau FHHNC pour lesquelles aucune mutation de la $C L D N 16$ n'avait été identifiée. Le tableau phénotypique comprenait l'hypomagnésémie, l'hypercalciurie avec une néphrocalcinose ; ce phénotype était associé chez quelques patients à des troubles oculaires ainsi qu'à d'autres signes tels que la cassure de la courbe de croissance, une polyurie ou encore une acidose tubulaire distale. Des nouvelles altérations moléculaires de la CLDN 19, ont pu être mises en évidence chez trois familles: la délétion des exons 1 à 4 et la mutation non sens p.Q57X, dans des familles consanguines et la mutation faux-sens p.P28L dans une troisième famille non consanguine. Ces mutations sont présentes à l'état homozygote, ce qui est en accord avec la transmission récessive de la maladie. La mutation faux-sens est très probablement pathogène, elle touche une proline localisée dans le premier domaine transmembranaire de la protéine. De plus, cet acide aminé est hautement conservé dans la famille des claudines de différentes espèces. Une atteinte oculaire (myopie sévère et strabisme) est retrouvée dans le cas index de la famille consanguine porteuse de la mutation p.Q57X. Dans les deux autres familles il n'existe pas de manifestations cliniques oculaires mais un examen ophtalmologique approfondi n'a pas été effectué. Cette étude confirme l'implication des gènes $C L D N 16$ et $C L D N 19$ dans l'FHHNC. L'absence de mutation dans six familles ayant un phénotype rénal semblable, est en faveur d'une hétérogénéité génétique supplémentaire.

Mots-clés : hypomagnésémie, claudines, néphrocalcinose.

DP394 / 334. SPECTRE DES MUTATIONS ET CORRÉLATIONS PHÉNOTYPE-GÉNOTYPE ASSOCIÉS À LA PARAPLÉGIE SPASTIQUE SPG11

G. Stevanin $(1,2)$, H. Azzedine $(1,3)$, P. Denora $(1,4)$, A. Boukhris $(1$, 2, 5), M. Tazir (6), A. Hamri (7), C. Goizet (1, 8), S. Forlani (1), M. Anheim (9), C. Depienne (1, 2), J. Truchetto (1), G. Garrigues (10), J. Vale (11), P. Coutinho (12), C. Tallaksen (13), A.L. Rosa (14), M. Tada (15), A. Lossos (16), F. Pasquier (17), F.M. Santorelli (4), C. Mhiri (5), A. Durr $(1,2), A$. Brice $(1,2)$ et le réseau SPATAX

(1) INSERM U679, Paris; (2) Département de Génétique et Cytogénétique, CHU Pitié-Salpêtrière, Paris : (3) Centre de Référence de Neurogénétique, CHU d'Angers; (4) Bambino Gesù Children's Hospital, Rome, Italie; (5) Hôpital Habib Bourguiba, Sfax, Tunisie ; (6) Hôpital Mustapha, Alger, Algérie ; (7) Hôpital Benbadis, Constantine, Algérie ; (8) Hôpital Pellegrin, Bordeaux ; (9) Hopital Civil, Strasbourg ; (10) CHU de Montpellier ; (11) Hospital De Egas Moniz, Lisbonne, Portugal ; (12) Hospital S. Sebastiao, Santa Maria da Feira, Portugal ; (13) Ullevål University Hospital, Oslo, Norvège; (14) Fundacion and Sanatorio Allende, Cordoba, Argentine ; (15) Niigata University, Japon ; (16) Hadassah-Hebrew University Medical Center, Jerusalem, Israel ; (17) CHRU de Lille, France

Introduction : Les paraparésies spastiques héréditaires sont des affections neurologiques cliniquement et génétiquement hétérogènes caractérisées par une faiblesse et une spasticité progressive des membres inférieurs parfois associées à d'autres signes neurologiques. Une entité clinique fréquente parmi les formes autosomiques récessives (PSAR) associe un léger retard mental, une polyneuropathie et une atrophie du corps calleux (PSARACC). La plupart des familles sont liées au locus SPG11 sur le chromosome 15 et nous avons récemment identifié 10 mutations tronquantes dans le gène KIAA1840, associées à ce phénotype. Matériel et méthodes: Afin de déterminer le spectre mutationnel et préciser le phénotype associé, nous avons analysé le gène KIAAI840 par séquençage direct ou analyse de liaison dans une grande cohorte de patients avec PSAR $(n=76)$ associant variablement $\mathrm{ACC}$, retard mental ou troubles cognitifs. Ces patients ont été collectés au sein du réseau européen et méditerranéen SPATAX.
Résultats : Nous avons identifié 22 mutations tronquantes transmises chez $7(21 \%)$ cas apparemment isolés et $13(30 \%)$ familles avec une transmission autosomique récessive. Dix-neuf de ces mutations sont nouvelles et incluent des mutations affectant l'épissage. Parmi les mutations connues, deux sont récurrentes et associées à des haplotypes partiellement conservés, suggérant des effets fondateurs au Portugal et en Afrique du Nord. La fréquence des mutations varie en fonction du phénotype des patients testés. La fréquence la plus élevée a été retrouvée chez les patients présentant une PSAR avec ACC (41\%), comme attendu. Ces mutations sont rares chez les patients avec retard mental sans ACC $(4,5 \%)$. L'âge de début des premiers signes (essentiellement troubles de la marche et/ou retard mental) varie de la première à la troisième décade. Après $14,9 \pm 6,6$ ans d'évolution, le phénotype des 38 cas examinés est sévère puisque $53 \%$ d'entre eux sont en chaise roulante ou confinés au lit. De plus, $80 \%$ des patients présentent une détérioration cognitive et une neuropathie. L'IRM cérébrale montre une ACC chez $95 \%$ des patients, une atrophie corticale chez $81 \%$ d'entre eux et des anomalies de la substance blanche chez $69 \%$. Conclusion: Cette étude montre la fréquence importante des mutations du gène SPGII parmi les patients présentant une ACC avec retard mental ou troubles cognitifs, neuropathie et anomalies de la substance blanche. Bien que plus fréquente chez les cas avec transmission autosomique récessive, SPG11 peut également rendre compte de cas apparemment sporadiques.

Mots-clés : paraplégie spastique, corps calleux, retard mental.

\section{-P395 / 347. DUPLICATIONS D'EXONS DANS LE GENE HSDI7B3 : UN NOUVEAU TYPE DE MUTATION RESPONSABLE DE DÉFICICIT EN 17BÊTA-HSD}

L. Michel-Calemard (1), E. Charmandari (3), J. Acherman (4), N. Skordis (5), Y. Morel $(1,2)$

(1) Hospices Civils de Lyon, Endocrinologie Moléculaire et Maladies Rares, Centre de Biologie et de Pathologie Est, Bron, F69677, France; (2) Université Lyon 1, Faculté de médecine Laënnec, Lyon, F69003, France; (3) The Middlesex Hospital, London, UK ; (4) Paediatric \& Adolescent Endocrinology, Institute of Child Health, London, UK ; (5) Paediatric Endocrine Unit, Makarios Hospital, Nicosia, Cyprus

Le déficit en 17bêta-Hydroxystéroöde déshydrogénase (17bêtaHSDde type 3) est une maladie génétique rare, de transmission autosomique récessive, responsable de « $46, X Y$ DSD (Disorders of Sexual Developement) » par anomalie testiculaire de la dernière étape de la biosynthèse de la testostérone. Seuls les sujets $46, \mathrm{XY}$ sont atteints et présentent une hypo-masculinisation des organes génitaux externes pouvant aller jusqu'à un phénotype féminin. L'hormone anti-mullërienne est normale car les testicules sont bien différenciés. En l'absence de gonadectomie, une virilisation pubertaire survient grâce à la conversion périphérique de la delta4-androstènedione (delta4A) en testostérone $(T)$ par une isoenzyme (17bêtaHSD de type V). Le gène HSD17B3, composé de 11 exons, code pour une enzyme purement testiculaire catalysant la réduction NADPHdépendante de la delta4A en T. Population étudiée: Parmi notre grande cohorte comprenant plus de 500 patients « 46,XY DSD » avec AMH normale, tous les patients présentant un ratio delta $4 / T>1$ après stimulation (à la naissance, la minipuberté ou après hCG), ont bénéficié d'un séquençage du gène HSD17B3. 49 patients présentant un déficit en 17bêtaHSD, ont ainsi été génotypés : 47 portaient 2 mutations (homozygotes ou hétérozygotes composites) et seuls 2 patients étaient simplement hétérozygotes. Matériel et méthodes : Pour l'un des 2 patients ci-dessus, l'ARNm testiculaire a pu être étudié à la suite d'une gonadectomie. Après RT-PCR et séquençage, une duplication hétérozygote de l'exon 2 était suspectée. Etant donné la difficulté d'obtention de l'ARNm, nous avons décidé de développer une technique permettant la détection des larges réarrangements à partir de l'ADN génomique. Une PCR multiplex fluorescente semi quantitative a été mise au point. Les 11 exons du gène HSDI7B3, ainsi que des amplicons contrôles, ont été co-amplifiés et déposés sur un séquenceur capillaire ABI 3100 après 18 cycles de PCR. Un quotient de dosage, comparant la hauteur des pics de tous les exons du patient et d'un sujet contrôle, a été calculé. La duplication de l'exon 2 a ainsi pu être confirmée chez notre patient et retrouvée chez son père. Cette stratégie a été appliquée à notre $2^{\mathrm{c}}$ patient avec une seule mutation identifiée sur son allèle maternel et a permis la détection d'une duplication hétérozygote s'étendant des exons 3 à 10 chez le patient et son père. Conclusion : Il s'agit de la première description de larges lésions du gène HSDI7B3. Leur recherche devrait être proposée chez tous les patients ayant une seule mutation identifiée ou ayant un profil hormonal typique d'un déficit en 17bêtaHSD.

Mots-clés : déficit en 17bêtaHSD, PCR multiplex fluorescente semi-quantitative, duplications d'exons. 
-P396/349. ANALYSE DU GÈNE CACNA1A CHEZ 25 INDEX SUSPECTS D'ATAXIE ÉPISODIQUE DE TYPE 2

\section{F. Riant $(1,2)$, C. Ploton (1), E. Tournier-Lasserve $(1,2)$}

(1) Laboratoire de Génétique Moléculaire, APHP Hôpital Lariboisière, Paris ; (2) INSERM U740, Paris

Introduction: L'Ataxie Episodique est une pathologie rare, hétérogène génétiquement, caractérisée par la survenue de crises paroxystiques avec troubles de l'équilibre. L'Ataxie Episodique de type 2 (EA2) a une transmission autosomique dominante et se caractérise par des crises durant de quelques minutes à plusieurs heures. Un nystagmus intercritique est classiquement présent. Certains patients développent une ataxie progressive et une atrophie vermienne. Les accès sont classiquement sensibles à l'acétazolamide. D'autres manifestations neurologiques paroxystiques (diplopie, épilepsie...) ou permanentes (retard mental...) peuvent s'y associer. Les premiers signes cliniques apparaissent généralement dans l'enfance mais peuvent survenir à l'âge adulte. Le diagnostic, basé sur la description clinique des crises peut être difficile en cas d'atypies. Des mutations ponctuelles du gène CACNAIA, le plus fréquemment de type perte de fonction, ont été identifiées dans de nombreux cas. Cependant, dans la littérature comme dans notre expérience, aucune mutation n'est identifiée chez certains patients malgré un séquençage complet du gène, soulevant l'hypothèse de l'existence de mutations d'une autre nature ou d'une hétérogénéité génétique. Objectifs : Déterminer la localisation et la nature des mutations sur une série de patients consécutifs avec suspicion d'EA2, en incluant la recherche de délétion, et comparer les cas avec et sans mutation. Matériel et méthode : 25 index non apparentés atteints d'EA2 ont été analysés, 8 cas familiaux et 17 cas sporadiques. Le criblage des 47 exons a été fait par séquençage et QMPSF. Cinq items cliniques ont été recueillis : le caractère familial ou sporadique, l'âge de début, la présence de nystagmus intercritique, les signes associés, la réponse à l'acétazolamide. Résultats : 13 mutations différentes ont été identifiées chez 14 index ( 6 familiaux, 8 sporadiques), incluant 12 mutations ponctuelles et 1 délétion emportant les exons 38,39 et 40 ; il s'agit de la première mutation de ce type rapportée dans l'EA2. Parmi les mutations ponctuelles, 9 sont de type perte de fonction, les autres sont 2 mutations faux sens touchant des acides aminés conservés (G13R et E147K) et une délétion de 3 nucléotides (p.A1593_Y1594delinsD). Elles n'ont pas été retrouvées chez 200 chromosomes contrôles. La mutation E147K a déjà été rapportée dans une famille associant épilepsie-absence et épisodes ataxiques. L'analyse clinique des 9 cas avec mutation de type perte de fonction montre i) l'existence d'une non réponse à l'acétazolamide chez plusieurs cas, ii) la présence d'un nystagmus intercritique chez tous les sujets. Aucune atypie n'est présente chez ces cas hormis des épisodes d'hyperammoniémie post crise chez un index et son apparenté muté. L'âge de début moyen est de $24.5 \pm 18.3$ ans chez les cas mutés, $(19,8 \pm 14,4$ pour les cas avec perte de fonction), et $39.5 \pm 25.6$ chez les non mutés. Trois cas sans mutation identifiée sont indistinguables des cas avec mutation perte de fonction, hormis 1 cas avec absence de nystagmus intercritique. La présence d'une atypie de type oscillopsie a été rapportée chez 2 cas non mutés. Conclusion - discussion: La découverte d'une délétion de grande taille rend nécessaire la recherche systématique de ce type de mutation en pratique diagnostique. La fréquence et la récurrence de ces délétions restent à déterminer sur une plus grande série. Une résistance à l'acétazolamide, un début tardif même chez des cas sporadiques peuvent être observés dans des cas avec mutation perte de fonction L'interprétation des mutations faux sens reste difficile à l'exception des cas de novo. Certaines mutations faux sens pourraient être plus souvent associées à un début tardif ou à des signes cliniques associées de type épilepsie-absence. Enfin, il existe des cas typiques sans mutation identifiée, en faveur d'anomalies régulatrices ou d'une hétérogénéité génétique.

Mots-clés : ataxie épisodique CACNAIA délétion.

P397 / 351. SYNDROME D'AARSKOG-SCOTT : ÉTUDE CLINIQUE ET ANALYSE MOLÉCULAIRE CHEZ 11 PATIENTS

N. Ronce (1, 2), A. Paubel (1), B. Doray (3), N. Philip (4), S. Jacquemont (5), A. David (5), H. Journel (6), A. Dieux (7), C. Vincent-Delorme (7, 8), A. Moncla (4), D. Martin-Coignard (9), F. M'Bou (10), V. Biancalana (3), M.-P. Moizard (1, 2), C. Moraine (2), M. Raynaud (1, 2)

(1) CHRU de Tours, Laboratoire de Génétique Moléculaire, Service de Génétique, 37000 Tours, France; (2) INSERM U619, 37000 Tours, France ; (3) CHRU de Strasbourg, Consultation de génétique et Laboratoire de Diagnostic Génétique, 67000 Strasbourg, France ; (4) CHU de Marseille, Département de Génétique Médicale, 13000 Marseille, France ; (5) $\mathrm{CHU}$ de Nantes, Génétique Médicale, 44000 Nantes, France ; (6) $\mathrm{CH}$ de Vannes, Génétique Médicale, 56000 Vannes, France; (7) CHRU de
Génétique Médicale, 62000 Arras, France ; (9) CH de Le Mans, Génétique Médicale, 72000 Le Mans, France: (10) CH du Lamentin, Service de Pédiatrie et Néonatalogie, 97292 Lamentin, Martinique, France

Le syndrome d'Aarskog-Scott ou dysplasie facio-digito-génitale se caractérise par une dysmorphie faciale, des anomalies des extrémités, des anomalies génitales et une petite taille. Ces signes peuvent être associés à un léger déficit intellectuel et/ou des troubles du comportement. Le gène en cause dans la forme liée à l'X (MIM\# 305400), FGDl (faciogenital dysplasia type 1) code une protéine GEF (guanine nucleotide exchange factor) qui active la protéine p21 GTPase Cdc42, un membre de la famille Rho des GTPases. La voie de signalisation FGD1/Cdc42 est impliquée dans l'organisation du cytosquelette d'actine et intervient dans la morphogénèse embryonnaire. La recherche de mutation du gène $F G D I$ a été réalisée dans une population de 25 garçons non apparentés présentant un tableau clinique évocateur. 11 mutations différentes (dont 1 déjà décrite) ont été identifiées : 4 faux-sens, 1 non-sens, 5 petites délétions et 1 mutation d'épissage. 7 des 11 mutations aboutissent à un arrêt de la traduction de la séquence codante et probablement à une perte de fonction de la protéine FGDI. Les données phénotypiques de chaque patient ainsi que les mutations associées sont décrites.

Mots-clés : Syndrome d'Aarskog-Scott, FGD1, Retard mental.

EP398 / 352. DYSTONIE LIÉE AU GÈNE TORIA : BILAN DANS LA POPULATION FRANÇAISE

M.Y. Frédéric (1, 2), F. Clot (3, 4, 5, 6), L. Cif (7), A. Blanchard (1, 2), A. Dürr $(3,4,5,6)$, I. Vuillaume $(8,9)$, G. Lesca $(10,11)$, A. Kreisler $(12,13)$, C. Davin $(1,2), T$. Besnard $(1,2)$, F. Rousset $(1,2)$, D. Thorel (14), C. Saquet (14), D. Mechin (14), L. Ozelius (15), Y. Agid (13, 4, 5, $16,17)$, B. Barroso (18), B. Chabrol (19), V. Chan (20), M. Clanet (21), C. Coubes (22), A. Destee $(12,13), \mathrm{K}$. Nguyen (23), C. Vial (24), M. Vidailhet $(3,4,5,16,17)$, J. Xie (25), B. Sablonnière $(8,9)$, A. Calender $(10,11)$, A. Brice $(3,4,5,6,16,17)$, A. Roubertie (26), P. Coubes (5), M. Claustres $(1,2,14)$, S. Tuffery-Giraud $(1,2)$, G. Collod-Beroud $(1,2)$ (1) INSERM, U827, Montpellier, F-34000 France ; (2) Université MONTPELLIERI, UFR Médecine, Montpellier, F-34000 France ; (3) INSERM, U679, Paris, F-75013 France; (4) Université Pierre-et-Marie-Curie, Paris-6, UMR S679, Hôpital de la Pitie-Salpêtrière, Paris, F-75013 France; (5) Institut Fédératif des Neurosciences (IFR70), Hôpital de la Pitié-Salpêtrière, Paris, France: (6) AP-HP, Groupe Hospitalier PitiéSalpêtrière, Département de Génétique cytogénétique et Embryologie, Paris, F-75013 France ; (7) CHU Montpellier, Hôpital Guy-de-Chauliac, Service de Neurochirurgie, Montpellier, F-34000 France ; (8) CHRU de Lille, Institut de Biochimie et Biologie Moléculaire, Lille, F-59037 France; (9) INSERM, U837, Lille, F-59045 France; (10) Hôpital Édouard-Herriot, service de Génétique Moléculaire et Clinique, Lyon, F-69437 France; (11) Université Claude-Bernard Lyon-1, Villeurbanne, F-69622 France; (12) CHRU de Lille, Service de Neurologie et pathologie du mouvement (EA2683), Lille, F-59037 France ; (13) Université de Lille-2 Droit et Santé, UFR Medecine, Lille, F-59045 France ; (14) CHU Montpellier, Hôpital Arnaud-de-Villeneuve, Laboratoire de Génétique Moléculaire, Montpellier, F-34000 France; (15) Department of Genetics and Genome Sciences, Mount Sinai School of Medicine, New York, NY, United States : (16) AP-HP, Groupe Hospitalier Pitié-Salpêtrière, Fédération de Neurologie, Paris, F-75013 France ; 15: INSERM, U289, Paris, F-75013 France: (17) Université Pierre et Marie Curie, Ecole Médicale de la Pitié-Salpêtrière, Paris, France; (18) CHU de Pau, Service de Neurologie, Pau, F-64046 France; (19) CHU de Marseille, Service de Neuropédiatrie, Marseille, F-13385, France, (20) CH de Valence, Service de Neurologie, Valence, F-26000 France ; (21) CHU de Toulouse, Hôpital Purpan, Service de Neurologie générale et Maladies inflammatoires du système nerveux, Toulouse, F-31059 France, (22) CHU Montpellier, Hôpital Arnaudde-Villeneuve, Service Génétique Clinique. Montpellier, F-34000 France ; (23) CHU de Marseille, Service de Génétique Médicale, Marseille, F-13385, France : (24) Hospices Civils de Lyon. Hôpital Neurologique et Neurochirurgical Pierre-Wertheimer, Service d'Électroneuromyographie et Pathologies Neuromusculaires, Lyon, F-69394, France ; (25) Hospices Civils de Lyon, Hôpital Neurologique et Neurochirurgical Pierre-Wertheimer, Service de Neurologie C, Lyon, F-69394, France ; (26) CHU Montpellier, Hôpital Guy-de-Chauliac, Service de Neuropédiatrie, Montpellier, F-34000 France

La dystonie est une maladie neurologique rare qui se manifeste par des contractions involontaires et soutenues affectant une ou plusieurs parties du corps, et entraînant des mouvements répétitifs de torsion et des postures anormales. Les dystonies primaires ou idiopathiques constituent un groupe de pathologies cliniquement et génétiquement hétérogènes. Elles sont généralement de transmission autosomique dominante avec une pénétrance 
réduite. La forme $D Y T 1$, bien caractérisée, correspond à une forme généralisée de la maladie avec une apparition précoce. Elle débute dans l'enfance en atteignant le plus souvent les membres inférieurs puis progresse jusqu'à la forme généralisée. La particularité de cette pathologie est que la majorité des cas identifiés dans différentes populations est due à une mutation unique et récurrente, correspondant à une délétion de trois paires de bases (c.907delGAG) au sein du gène TORIA localisé sur le chromosome 9. En France, peu de données épidémiologiques sont rapportées dans la littérature quant aux porteurs de cette mutation. Une collaboration entre les 4 centres de référence pour le diagnostic moléculaire de la forme $D Y T l$ en France (Lille, Lyon, Paris et Montpellier) a été mise en place. Elle nous a permis de mettre en place le recensement de la population française porteuse de cette mutation et de caractériser l'haplotype associé à la délétion dans les différentes familles identifiées. Cette collaboration a permis d'identifier 53 cas index. Pour chacun, les données cliniques, moléculaires et l'ADN de l'ensemble des apparentés disponibles pour l'analyse ont été collectés. Au total, 108 individus porteurs de la mutation ont été identifiés dont 24 asymptomatiques. La fréquence de la mutation c.907delGAG a donc pu être estimée à au moins 0,17:100,000 dans la population française. Les haplotypes associés à la mutation ont été construits en analysant 5 microsatellites entourant le gène. L'haplotype particulier associé à un effet fondateur dans la population juive ashkénaze (JA) a été retrouvé dans 12 familles avec ou sans origine JA. Quatre familles présentent un second haplotype identifié dans la population JA. Parmi les 37 autres familles étudiées, seulement 2, sans aucun lien de parenté rapporté, partagent le même haplotype. Cette étude a permis de caractériser les porteurs de la mutation c.907delGAG du gène TORIA responsable de la forme généralisée de dystonie dans la population française. Les résultats confirment l'absence d'effet fondateur et la rareté de cette maladie en France.

Mots-clés : DYTI, dystonie primaire précoce, TORIA.

\section{IP399 / 370. CHORÉE DE HUNTINGTON ASYMPTOMATIQUE} ASSOCIÉE À UNE DÉGÉNÉRESCENCE CORTICO-BASALE

D. Caparros-Lefebvre (1), O. Kerdraon (2), C.M. Dhaenens (3, 4), C.A. Maurage (2), A. Delacourte (4), B. Sablonnière $(3,4)$

(1) Service de Neurologie, $\mathrm{CH}$ de Valenciennes; (2) Laboratoire de Neuropathologie, Centre de Biologie-Pathologie; (3) UF de Neurobiologie, Centre de Biologie-Pathologie, CHRU de Lille ; (4) INSERM U837, Centre de Recherches Jean-Pierre-Aubert, Université de Lille 2

Présentation clinique. Une patiente de 71 ans, sans antécédent personnel ni familial notable, développa une dysarthrie rapidement progressive, associée à une dysphagie en rapport avec une apraxie oro-bucco-linguale. S'y associait un syndrome cérébelleux axial modéré et peu évolutif, avec hypotonie cérébelleuse et embardées au demi-tour. L'évaluation cognitive révélait de discrets troubles praxiques. Après 6 mois d'évolution, apparaissai un syndrome pyramidal qui restait limité aux muscles d'innervation bulbaire. Puis, la dysphagie devint prédominante alors que la patiente étai anarthrique. Dans les dernières semaines de vie, le syndrome pyramidal s'étendait aux membres avec amyotrophie, fasciculations et déficit moteur. L'état cognitif était préservé. Tout au long de la maladie, la patiente n'a jamais présenté de mouvement choréique. Une détresse respiratoire survenait 22 mois après le début des manifestations cliniques et entraînait le décès. L'IRM encéphalique était normale et l'imagerie fonctionnelle par SPECT montrait une hypoperfusion fronto-pariéto-temporale bilatérale. Cliniquement, l'implication du cervelet, du cortex pariétal et des voies pyramidales dans un processus dégénératif rapidement progressif faisai évoquer en premier lieu une dégénérescence cortico-basale, en l'absence de syndrome frontal évident et de trouble de l'oculomotricité. Neuropathologie : Macroscopiquement, le cerveau présentait une atrophie corticosous-corticale modérée prédominant au niveau fronto-temporal et épargnant relativement les noyaux gris centraux. Les lésions microscopiques associaient une perte neuronale, une spongiose laminaire corticale et une gliose astrocytaire, principalement au niveau temporal antérieur. L'immunohistochimie montrait des lésions de Tauopathie au niveau neuronal (dégénérescence neurofibrillaie hippocampique) et glial (corps bobinés), sans dépôt amyloïde, dont la distribution évoquait une dégénérescence cortico-basale. En plus de ces lésions de dégénérescence cortico-basale, les anticorps anti-huntingtine révélaient des agrégats caractéristiques de maladie de Huntington, de grade 1 selon la classification de Vonsattel. Biochimie. La présence de dégénérescence neurofibrillaire fut observée à partir d'homogénats de différentes aires cérébrales (noyau de Meynert, Hippocampe, cortex temporal, pallidum inyerne et noyau sous-thalamique) par la présence d'agrégats de protéines tau pathologiques révélées par l'anticorps AD2. Le profil électrophorétique indiquait la présence d'un doublet de protéines Tau ( 69 et $64 \mathrm{kDa})$, caractéristique du profil observé dans la dégénérescence cortico-basale. Génétique: En présence d'une hypotonie, d'un syndrome cérébelleux associé à quelques mouvements anormaux erratiques, le test génétique de la chorée de Huntington était pratiqué. Une expansion de 40 motifs CAG fut observée. Ce résultat fut confirmé par la mesure de la taille de la répétition du motif CAG à partir d'un extrait de tissu cérébral. Discussion : Cette observation souligne la difficulté posée parfois par le diagnostic de maladies neuro-dégénératives dont la présentation clinique n'oriente pas vers une maladie héréditaire. En l'absence d'histoire familiale de chorée de Huntington dans la famille, cette présentation objective un cas de Huntington quasiment asymptomatique, chez une femme de 71 ans dont la taille de l'expansion correspond à un risque de $50 \%$ de développer les signes cliniques à 60 ans et de $93 \%$ à 70 ans. Il peut s'agir d'une association fortuite de deux maladies neurodégénératives. Cependant l'absence de signes d'appel francs en faveur d'une chorée de Huntington permet de supposer un retard de l'évolution de la pathogénie de I'huntingtine liée à une dégénérescence neurofibrillaire. Ainsi une intrication des mécanismes pathogéniques entre l'huntingtine mutée et les protéines tau n'est pas à exclure, mais reste à démontrer.

Mots-clés : Huntington atypique, dégénérescence neuronale, clinique.

- 400 / 372. COLAA1, UN NOUVEAU GÈNE IMPLIQUE DANS LES MALFORMATIONS D'AXENFELD-RIEGER SYNDROMIQUES

I. Coupry (1), I. Sibon (2), I. Orignac (1), I. Burgelin (1), J.-M. Orgogozo (2), D. Lacombe $(1,3)$, B. Arveiler $(1,3)$, C. Goizet $(1,3)$

(1) Laboratoire de Génétique Humaine, Université Victor-Segalen, Bordeaux 2, 33076 Bordeaux; (2) Fédération de Neurosciences Cliniques : (3) Service de Génétique Médicale, Hôpital Pellegrin, CHU Bordeaux, 33076 Bordeaux

Introduction: Le terme «malformation d'Axenfeld-Rieger » (MAR) s'applique à une constellation de malformations de la chambre antérieure de l'œil appartenant au groupe hétérogène des dysgénésies du segment antérieur. Les MAR peuvent être isolées ou bien associées à d'autres malformations dans un cadre syndromique. À ce jour, seuls trois gènes codant pour des facteurs de transcription (PITX2, PAX6 et FOXC1) avaient été impliqués dans les mécanismes moléculaires à l'origine de MAR Sujets et Méthodes Cinq apparentés, porteurs d'une MAR associée à une leucoencéphalopathie et répartis sur trois générations successives, ont été investigués sur le plan clinique et génétique. Résultats La leucoencéphalopathie traduit l'existence d'une vasculopathie cérébrale chez tous les sujets atteints, pouvant être à l'origine d'accidents vasculaires cérébraux, y compris du sujet jeune, mais pouvant également être asymptomatique. Les analyses de liaison dirigées sur plusieurs loci impliqués dans les MAR ou bien dans les vasculopathies cérébrales ont révélé une association possible avec les loci 11p13 (PAX6) et 13q34 (COLAAI). Le séquençage direct de COLAA1 a révélé une nouvelle mutation faux-sens, p.Gly720Asp. Conclusion: Nous décrivons une nouvelle forme syndromique de MAR. Nos résultats confirment que les mutations de COLAAl peuvent causer des MAR chez l'homme conformément aux observations déjà faites chez la souris.

Mots-clés : Axenfeld-Rieger, COLAA1, leucoencéphalopathie.

घP401 / 374. MALADIE DE MENKES ET SYNDROME DES CORNES OCCIPITALES : IDENTIFICATION DE NOUVELLES MUTATIONS

M.P. Moizard (1, 2), N. Ronce (1, 2), N. Marmin (1), A. Paubel (1), S. Mercier (1), S. Blesson (1), A. Toutain (1), C. Moraine (2), M. Raynaud $(1,2)$

(1) Service de Génétique, CHRU de Tours ; (2) INSERM U 619, Tours

La maladie de Menkes est un trouble sévère du métabolisme du cuivre, de transmission récessive liée à l'X, qui touche un garçon sur 250000 . Elle se caractérise cliniquement dès les premiers mois de vie par un retard de croissance, une détérioration neurologique progressive et une atteinte des tissus conjonctifs associés à un aspect caractéristique de la peau et des cheveux. Les taux de cuivre sérique et de céruloplasmine sont effondrés. Le décès survient dans la majorité des cas avant l'âge de 3 ans. Il existe des formes plus modérées dont le syndrome des cornes occipitales (OHS) qui se manifeste essentiellement par l'atteinte du tissu conjonctif. Ces différentes formes cliniques sont liées à des mutations du gène ATP7A qui comporte 23 exons et qui code pour une ATPase de type P de transport intracellulaire du cuivre de 1500 acides aminés. Un défaut de la protéine entraîne un déficit en cuivre libre et conduit à l'altération de la fonction des enzymes cuivre dépendantes, à l'origine les différents symptômes de la maladie. Les mutations décrites à ce jour sont majoritairement des mutations ponctuelles de tout type et des délétions qui emportent un à plusieurs exons du gène. L'absence de point chaud de mutation implique, pour 
chaque patient, l'analyse de la totalité du gène (séquence codante de 8122 $\mathrm{pb})$. Nous avons développé pour cela deux approches moléculaires complémentaires : l'une sur ADN génomique qui utilise les techniques de PCR séquençage et PCR semi quantitative des 23 exons, l'autre sur ARNm extrait de lignée lymphoblastoide ou de culture de fibroblastes. À ce jour, 39 patients ( 35 cas index dont 1 présentant un phénotype OHS, 2 sujets féminins présentant des signes cliniques et 2 apparentées de sujets masculins décédés, conductrices par la généalogie) ont été étudiés. L'analyse a permis de mettre en évidence une mutation chez 33 patients soit 32 mutations réparties en 3 petites mutations de décalage de phase, 4 mutations non-sens, 5 mutations d'épissage, 9 mutations faux-sens et 11 grands réarrangements exoniques. Pour les familles avec mutation identifiée, ces résultats ont permis de confirmer le diagnostic clinique, de proposer la réalisation d'un diagnostic prénatal par méthode directe (10 ont été réalisés) et de renseigner plusieurs apparentées à risque sur leur statut.

Mots-clés : Menkes, OHS, ATP7A.

\section{P402 / 376. UN ÂGE DE DÉBUT PRÉCOCE EST LE CRITÈRE CLINIQUE MAJEUR DE LA MALADIE DE PARKINSON LIÉE AUX MUTATIONS DU GÈNE PARK2}

C. Cazeneuve (1), C. Sân (1), E. Lohmann (2), E. Leguern (1, 2), A. Dürr $(2,3)$, A. Brice $(1,2,3)$, et le groupe Parkinson Disease Genetics

(I) Dpt de Génétique, UF de Neurogénétique Moléculaire et Cellulaire, Groupe Hospitalier Pitié-Salpêtrière, AP-HP, 75013 Paris ; (2) Inserm U679, Groupe Hospitalier Pitié-Salpêtrière, 75013 Paris; (3) Dpt de Génétique, UF de Génétique Clinique, Groupe Hospitalier Pitié-Salpêtrière, AP-HP, 75013 Paris

Introduction : La forme juvénile autosomique récessive de la maladie de Parkinson (JPD) associe les signes habituels de la maladie de Parkinson (akinésie, rigidité, tremblement de repos) à une bonne réponse à la L-DOPA, une évolution lente et un âge de début précoce ( $<40$ ans). Le gène le plus fréquemment en cause dans la JPD est le gène PARK2. Une recherche des mutations du gène PARK2 est assez souvent proposée aux patients ayant développé la maladie entre 40 et 60 ans et se présentant comme des cas sporadiques ou compatibles avec une transmission autosomique récessive. Patients et Méthodes : Afin de préciser les critères cliniques de la JPD associée à $P A R K 2$, nous présentons ici le résultat de l'analyse moléculaire du gène PARK 2 chez 123 patients (soit 122 familles) ayant un âge de début compris entre 11 et 57 ans et un diagnostic clinique de maladie de Parkinson. La recherche de réarrangement a été réalisée par Multiplex Ligation-dependent Probe Amplification (MLPA kits P051/P052, MRC-Holland; ces kits détectent également la mutation p.Gly2019Ser du gène $L R R K 2$, associée à des formes dominantes avec pénétrance incomplète de la maladie de Parkinson et les réarrangements du gène $S N C A$, également associé à des formes dominantes); les mutations ponctuelles ont été recherchées par séquençage direct des 12 exons du gène et de leurs régions introniques flanquantes. Résultats-Discussion : Au total, $52 \%(23 / 44)$ des mutations identifiées du gène PARK2 sont des réarrangements (délétion ou multiplication d'un ou plusieurs exons); les mutations ponctuelles sont des mutations faux-sens $(n=12)$ ou induisant un décalage de cadre de lecture $(\mathrm{n}=9)$. Vingt patients, dont deux sœurs, portent deux mutations de $P A R K 2$ (patients $P A R K 2$ ), quatre portent une seule mutation et 92 ne portent aucune mutation de $P A R K 2$. Un patient porte une duplication du gène SNCA. La mutation G2019S est retrouvée chez les 6 patients restant dont deux portent également une mutation de $P A R K 2$. La distribution des génotypes PARK2 (2,1 ou 0 mutation) diffère très significativement de la loi de Hardy-Weinberg, le groupe des patients portant une seule mutation identifiée étant très largement sous-représenté. Ce résultat suggère fortement que le phénotype de la très grande majorité des patients chez lesquels aucune mutation n'a pu être identifíe n'est pas lié au gène $P A R K 2$ plutôt que lié à des mutations de $P A R K 2$ siégeant dans des régions non explorées. L'âge de début est inférieur à 40 ans chez tous les patients PARK2, l'âge de début moyen ( 24,5 ans) étant plus précoce que celui des autres patients $(36,1$ ans, $\mathrm{p}<0,001)$. Cependant, parmi les patients dont l'âge de début est inférieur à 40 ans, seuls $24 \%(20 / 83)$ présentent deux mutations identifiées du gène $P A R K 2$, leur âge de début étant également inférieur à celui des autres patients $(31,1$ ans, $\mathrm{p}<0,001)$. En revanche, le profil clinique des patients PARK2 (rigidité, akinésie, tremblement, réponse à la L-DOPA, début asymétrique) n'est pas significativement différent de celui des autres patients. Enfin, la proportion de patients PARK2 est plus élevée dans les familles autosomiques récessives $(54 \%, 7 / 13)$ que pour les cas sporadiques ou aux antécédents familiaux éloignés $(11 \%, 12 / 109)$ mais ces derniers représentent plus de $50 \%$ des patients PARK2. Conclusion Au total, ces résultats montrent qu'un âge de l'analyse moléculaire du gène PARK2 dans les formes de la maladie de Parkinson compatibles avec un mode de transmission autosomique récessif.

Mots-clés : Parkinson, critère clinique, PARK2.

\section{GP403 / 377. RÉDUCTION DE L'INTERVALLE CANDIDAT ET PHÉNOTYPE ASSOCIÉ À LA PARAPLÉGIE SPASTIQUE SPG15: ÉTUDE DE 8 FAMILLES}

P. Denora $(1,2)$, A. Boukhris $(1,3)$, N. Elleuch $(1,3)$, N. Bouslam $(1,4)$, S. Hanein (1), A. Lossos (5), A. Hamri (6), I. Feki (3), E. Denis (7), A. Benomar (4), C. Mhiri (3), C. Goizet (1), F.M. Santorelli (2), A. Brice (1, 7), G. Stevanin $(1,7)$

(1) INSERM/UPMC UMR679; (2) Bambino Gesù Children's Hospital, Rome, Italie; (3) Hôpital Habib Bourguiba, Sfax, Tunisie; (4) Hôpital des Spécialités, Rabat, Maroc; (5) Hadassah-Hebrew University Medical Center, Jerusalem, Israël; (6) Hôpital Benbadis, Constantine, Algérie ; (7) Département de Génétique et Cytogénétique du CHU Pitié-Salpêtrière, Paris

Introduction : La paraplégie spastique héréditaire (PSF) de type 15 est une forme rare de PSF récessive autosomique (RA) complexe, qui présente des caractéristiques phénotypiques très hétérogènes s'intégrant initialement au syndrome de Kjellin. Les signes les plus courants sont : paraplégie spastique de progression lente, retard mental suivi par une détérioration intellectuelle, amyotrophie distale, maculopathie et légers signes cérébelleux. Le locus responsable (SPG15) a été localisé sur le chromosome 14q22-q24, dans un intervalle de $19 \mathrm{~cm}$. Matériels et Méthodes: Huit familles, dont deux sont apparentées, ont été inclues dans notre étude. Ces familles présentaient un phénotype complexe comprenant une atteinte cognitive et certains signes associés au syndrome de Kjellin. Après exclusion par analyse de liaison des autres loci de RA-PSF connus (dont SPG11), nous avons réalisé une cartographie primaire dans trois grandes familles consanguines d'origine Arabe et l'analyse du locus SPG15 a été réalisée directement sur les cinq autres familles d'origine tunisienne et italienne en utilisant des marqueurs microsatellites flanquants. Résultats : Les huit familles se sont avérées liées au locus SPG15 $(2.0>Z \max >4.3)$ et la reconstitution des haplotypes nous a permis de réduire la taille de la région candidate de $\sim 16$ à $\sim 5 \mathrm{Mb}$, entre les markers D14S981 et rs8688. Les vingt individus atteints de ces huit familles montraient une paraplégie spastique (20/20) associée à une détérioration cognitive de progression sévère $(15 / 20)$, des légers signes cérébelleux $(3 / 20)$ et une neuropathie axonale et démyelinisante $(7 / 20)$. Un corps calleux fin n'a été retrouvé que dans quatre familles, et seulement deux patients présentaient des anomalies au fond de l'œil. L'âge de début variait de 8 à 19 ans. Le séquençage direct de deux des 50 gènes candidats contenus dans cette région (GPHN et SLC8A3, qui codent respectivement pour une protéine associée aux microtubules et pour un échangeur $\mathrm{Na}+/ \mathrm{Ca}++)$ n'a mis en évidence aucune mutation dans leurs exons et dans les régions UTRs. Conclusion: Nous rapportons la plus grande cohorte de patients SPG15, qui nous a permis de réduire la taille de l'intervalle candidat et de démontrer que ce locus peut être associé à des formes différentes du syndrome de Kjellin.

Mots-clés : paraplégie spastique, corps calleux, retard mental.

- 404 / 382. LE DIAGNOSTIC GÉNÉTIQUE MOLÉCULAIRE DU SYNDROME DU QT LONG CONGÉNITAL

V. Fressart (1), A. Le Hen (1), N. Benammar (1), I. Denjoy (2), J.M. Lupoglazoff (2), P. Charron (2), M. Berthet (3), P. Guicheney (3), B. Hainque $(1,3)$

(1) UF de Cardiogénétique et Myogénétique, Service de Biochimie Métabolique, Hôpital Pitié-Salpêtrière (AP-HP), Paris ; (2) Centre de Référence pour les Maladies Cardiaques Héréditaires, Paris; (3) INSERM U582, Institut de Myologie, Hôpital Pitié-Salpêtrière (AP-HP), Paris

Introduction : Le syndrome du QT long congénital (QTL) est une pathologie rythmique ventriculaire rare. Ce syndrome se caractérise à l'électrocardiogramme (ECG) de surface par un allongement de l'intervalle QT. La prévalence serait supérieure à 1/5000. Le syndrome du QTL congénital est cliniquement hétérogène : bradycardie sinusale, lipothymies, syncopes et aussi morts subites qui en font toute la gravité. Le diagnostic est porté classiquement chez des sujets jeunes. La transmission est habituellement autosomique dominante. En plus des formes dites congénitales, il existe des formes dites acquises, médicamenteuses ou liées à des désordres hydroélectrolytiques. Aspects génétiques. Le syndrome du QTL congénital se caractérise par une grande hétérogénéité génétique. Ainsi à ce jour, 8 gènes différents sont rapportés dans ce syndrome. Parmi eux, 3 gènes sont plus fréquemment impliqués, les gènes $K C N Q 1$ et $K C N H 2$ qui codent des canaux potassiques et le gène $S C N 5 A$ codant un canal sodique. De rares mutations ont été identifiées dans les gènes $K C N E 1$ et $K C N E 2$ qui codent 
des sous unité \&\#61538; de canaux potassiques. Seul le gène $A N K 2$ ne code pas pour un canal, mais pour une protéine intracellulaire, l'Ankyrine $\mathrm{B}$, dont la fonction serait l'ancrage de protéines à la membrane. Enfin, 2 autres gènes ont plus rarement été impliqués dans le syndrome du QTL. L'allongement de l'intervalle QT est alors associé à des dysmorphies ou des malformations. Il s'agit du gène $K C N J 2$, codant un canal potassique impliqué dans le syndrome d'Andersen et du gène CACNAIC, codant un canal calcique, impliqué dans le syndrome de Thimothy. But de l'étude. Le but de cette étude était d'évaluer l'efficacité de l'analyse des gènes les plus fréquemment impliqués dans le syndrome du QTL congénital. Méthodes: Nous avons reçu au laboratoire 1419 prélèvements dont 416 patients index et 1003 apparentés. Nous avons analysé les patients index sur 1 ou 2 ou 3 des gènes les plus fréquemment impliqués dans le syndrome du QTL. En effet, en l'absence de mutation sur le premier gène étudié, l'analyse se poursuit sur le second puis en dernier lieu sur le troisième (l'ordre de priorité est fonction du tableau clinique). Résultats. Nous avons identifié à ce jour des mutations chez 145 cas index, mais toutes les analyses ne sont pas terminées. 72 familles sont porteuses de 54 mutations dans $K C N Q 1$. Ces mutations sont des mutations faux sens $(72 \%)$, des délétions et/ou insertions $(16 \%)$, des non sens $(8 \%)$ et des mutations d'épissage ( $4 \%$ ). 67 familles sont porteuses de 59 mutations dans $K C N H 2$. Parmi ces mutations, $64 \%$ sont des faux sens, $22 \%$ des délétions et/ou insertions, $12 \%$ des non sens et $2 \%$ des mutations d'épissage. 6 familles présentent une mutation unique dans $S C N 5 A$ ( 5 faux sens et 1 délétion de 3 nucléotides). En plus des cas index, l'analyse des apparentés a permis de rendre le diagnostic génétique à 799 patients (145 cas index et 654 apparentés) dans ces 145 familles. La présence d'une mutation a été mises en évidence chez 394 sujets (221 porteurs de mutation dans $K C N Q 1,146$ mutés dans $K C N H 2$ et 27 mutés SCN5A). Les mutations n'ont pas été retrouvées chez 405 sujets (190 indemnes issus de familles présentant une mutation KCNQ1, 184 sujets issus de familles atteintes de mutation $K C N H 2$ et 31 au sein des familles mutées dans SCN5A). Conclusions : En accord avec la littérature, les mutations causales identifiées dans le cadre du syndrome du QTL, le sont majoritairement dans KCNQI $(50 \%)$ et KCNH2 (46\%). Dans notre cohorte de familles mutées, les mutations dans $S C N 5 A$ sont rares et représentent moins de $5 \%$ des mutations responsables du syndrome du QTL. L'indication de la recherche de mutation dans $S C N 5 A$ doit donc être étayée par des renseignements cliniques suggérant fortement l'implication de ce gène comme des syncopes au repos associées à un allongement marqué du segment ST. Au total, le diagnostic moléculaire a permis de confirmer le diagnostic de syndrome de QTL chez $49 \%$ de notre population.

Mots-clés : syndrome du QTL, diagnostic génétique, arythmie ventriculaire.

\section{- P405 / 385. SPECTRE MUTATIONNEL DE LA MALADIE DE GAUCHER EN TUNISIE}

W. Cherif (1, 2), R. Kefi-Ben Atig (1), H. Ben Turkia (2), N. Tebib (2), O. Amaral (3), F. Ben Rhouma (1), M. S. Abdelmoula (2), H. Azzouz (2), C. Caillaud (3), M.C. Miranda (4), S. Abdelhak (1), M.F. Ben Dridi (2) (1) Unité 26/04 d'Exploration Moléculaire des Maladies Orphelines d'Origine Génétique, Institut Pasteur de Tunis, Tunisie; (2) Unité 25/04 de Dépistage et de Prise en charge des Maladies Héréditaires du Métabolisme, Service de Pédiatrie du CHU la Rabta Tunis, Tunisie ; (3) Laboratoire de Génétique Métabolique, Faculté de Médecine de Cochin, France ; (4) Département de Neurobiologie Génétique, Institut de Biologie Moléculaire et Cellulaire, université de Porto, Portugal

Introduction : La maladie de Gaucher est la maladie métabolique la plus fréquente parmi les affections de surcharge lysosomale. Sa transmission est autosomique récessive. Elle est due à un déficit enzymatique en bétaglucosidase entraînant l'accumulation pathologique du glucosylceramide dans les lysosomes. Depuis la découverte du gène $G B A$ qui code pour la béta-glucosidase, plus de 200 mutations ont été rapportées parmi lesquelles les mutations récurrentes N370S, L444P et C.84insG. Leurs fréquences et leurs distributions sont variables selon les populations étudiées. Objectif : Les maladies héréditaires métaboliques sont des maladies rares mais en Tunisie, elles sont relativement fréquentes à cause du taux élevé de la consanguinité. Notre but est de déterminer le spectre mutationnel de la maladie de Gaucher en Tunisie et de développer un outil moléculaire de diagnostic pour cette pathologie. Matériel et méthodes: Nous avons recherché, par PCR / RFLP et séquençage direct, les mutations N370S, L444P, chez 11 enfants tunisiens, atteints de la maladie de Gaucher, appartenant à 10 familles non apparentées. Résultats : Chez les patients tunisiens, la mutation N370S est la plus fréquente ( $6 / 20$ allèles mutés, $30 \%)$, suivie par l'allèle recombinant (RecNcil) (5/20 allèles mutés, $25 \%)$. La mutation L444P présente une fréquence de l'ordre de $20 \%$ (4/20 allèles mutés). Parmi les patients étudiés, cinq sont hétérozygotes composites N370S/RecNciI. Conclusion : La recherche des mutations N370S et L444P par PCR/RFLP fournit un outil de diagnostic moléculaire simple, non invasif permettant un dépistage des hétérozygotes indispensable pour le conseil génétique.

Mots-clés : maladie de Gaucher, diagnostic moléculaire, gène GBA.

-P406 / 389. IDENTIFICATION D'UN CINQUIÈME GÈNE DE L'HYPERCHOLESTÉROLÉMIE FAMILIALE

M. Marduel, M. Abifadel, A. Marques, J. Bonneau, M. Devillers, D. Erlich, A. Munnich, J.P. Rabès, C. Boileau et M. Varret

INSERM UR781, Paris, France

L'hypercholestérolémie familiale est un facteur de risque majeur de l'athérosclérose dont les complications cardio-vasculaires sont la principale cause de morbidité et de mortalité dans les pays industrialisés. Les facteurs génétiques intervenant dans l'homéostasie du cholestérol sont certainement nombreux, mais l'identification des gènes dont les altérations sont associées à l'Hypercholestérolémie Familiale (HCF) a contribué à une meilleure compréhension des mécanismes de régulation du taux plasmatique de cholestérol. L'hypercholestérolémie à transmission autosomique dominante $(\mathrm{ADH})$ est l'une des maladies génétiques les plus fréquentes, touchant un sujet sur 500 sous sa forme hétérozygote. Elle se traduit par des concentrations élevées en LDL (Low Density Lipoprotein), lipoprotéines riches en cholestérol. L'excès de cholestérol provoque des dépôts extravasculaires entraînant la formation de xanthomes tendineux ou cutanés, de xanthélasmas, d'arcs cornéens, mais aussi des dépôts vasculaires au niveau de l'aorte, des artères coronaires, carotidiennes et fémorales à l'origine des plaques d'athérosclérose et des complications cardiovasculaires. Le premier gène impliqué dans I'HCF fut le gène codant le récepteur des LDL $(L D L R)$, mis en évidence par Brown et Goldstein en 1986. En 1987, les travaux de l'équipe d'Innerarity révélèrent le deuxième gène, $A P O B$, codant l'apolipoprotéine B-100 portée par les LDL et ligand des récepteurs des LDL. Puis, notre équipe a mis en évidence deux locus supplémentaires impliqués dans la maladie. Un troisième gène, PCSK 9 (Proprotein Convertase Subtilisin/kexin type 9), codant une sérine protéase, fut alors découvert en 2003. L'identification du quatrième gène, HCHOLA4, est en cours : il est localisé en $16 \mathrm{q} 22.1$ dans un intervalle de $5,31 \mathrm{~cm}$ et coderait une protéine impliquée dans le catabolisme des LDL. Grâce au Réseau de Recherche National de l'HCF, notre équipe a collecté du matériel génétique d'une grande famille, appelée HC126. Vingt-six échantillons furent obtenus de cette famille multiplexe, dont 16 provenant de sujets atteints, et 10 provenant de sujets normocholestérolémiques. Des analyses par haplotypage et séquençage de la famille $\mathrm{HC} 126$ ont permis d'exclure l'implication des gènes $L D L R, A P O B$ et $P C S K$ 9. De plus, l'étude de 6 marqueurs microsatellites recouvrant l'intervalle $H C H O L A 4$ a permis d'exclure clairement cette région pour la famille $\mathrm{HC126}$. Ces résultats démontrent l'existence d'un cinquième gène de l'HCF : HCHOLA5. Pour évaluer la puissance de la famille $\mathrm{HCl} 26$ pour le linkage, 500 simulations furent effectuées avec les génotypes grâce au logiciel SLINK en utilisant des paramètres compatibles avec l'ADH. L'espérance de LOD score obtenue fut de 2,08 et le maximum de LOD Score attendu fut de 3,96, ce qui signifiait que le seuil statistiquement significatif de 3 pouvait être atteint avec cette famille unique. Le tour du génome effectué en collaboration avec le Centre National de Génotypage a fait ressortir 5 régions à forts LOD score. Sur l'une d'elles, une étude par haplotypage a permis de réduire une zone de 6,4 mégabases comprenant environ 160 gènes, dont un, particulièrement intéressant de part son implication dans le métabolisme du cholestérol, qui est en cours d'étude par séquençage. Pour valider cette région comme contenant effectivement $H C H O L A 5$, nous avons accès à du matériel génétique provenant de petites familles présentant une HCF non liée aux gènes $L D L R, A P O B$ et PCSK9. Nous effectuons l'haplotypage de ces petites familles afin de réduire encore plus la zone d'intérêt. L'identification du gène $H C H O L A 5$ devrait permettre de comprendre encore plus précisément le métabolisme du cholestérol, voire d'en révéler un aspect nouveau, ce qui pourrait permettre le développement de nouvelles méthodes préventives, ou de médicaments mieux ciblés.

Mots-clés : hypercholestérolémie, linkage, gène candidat.

EP407 / 391. PERTURBATIONS DES PROCESSUS BIOLOGIOUES SUITE À L'ACTIVATION CONSTITUTIVE DE FGFR3: ANALYSE TRANSCRIPTOMIQUE DE CULTURES PRIMAIRES DE CHONDROCYTES HUMAINS

L. Schibler (1), L. Gibbs (1), C. Benoist-Lasselin (1), C. Decraene (2), A. Lise Delezoide (3), M. Gonzales (4), J. Martinovic (5), P. Loget (6), A. Munnich(1), L. Legeai-Mallet (1) 
(1) INSERM U781-Département de génétique, Hôpital Necker, Paris ; (2) Plateforme de Génomique Fonctionnelle Curie, Hôpital Saint-Louis Paris ; (3) Service d'Anatomopathologie, Hôpital Robert-Debré, Paris ; (4) Service de Génétique et d'Embryologie Médicale, Hôpital Trousseau, Paris ; (5) Service de Fatopathologie, Hôpital Necker, Paris ; (6) Centre Pluridisciplinaire de Diagnostic Prénatal de Rennes, CHU de Rennes

L'achondroplasie, le nanisme le plus fréquent chez l'homme, fait partie d'un groupe de chondrodysplasies de sévérité variable incluant l'hypochondroplasie, la forme modérée et le nanisme thanatophore, la forme létale. Ces dysplasies squelettiques dont le mode de transmission est autosomique dominant se caractérisent par des membres courts, des anomalies vertébrales et une macrocéphalie plus ou moins marquée. Le gène responsable, FGFR3, est un récepteur transmembranaire à activité tyrosine kinase qui régule négativement la croissance osseuse. Les diverses mutations décrites dans ce gène entraînent son activation constitutive, se traduisant par une maturation accélérée et défectueuse des chondrocytes de la plaque de croissance. À l'heure actuelle, le rôle exact de FGFR3 au niveau moléculaire est encore mal compris. FGFR3 utilise préférentiellement la voie des MAPKinases, le récepteur muté active également la voie de signalisation des STATs. L'activation constitutive du récepteur provoque un arrêt prématuré du cycle cellulaire des cellules prolifératives, qui se différencient précocement en chondrocytes préhypertrophiques. D'autres voies interviennent probablement et différentes molécules telles Ihh, PTHrP ou BMP sont potentiellement impliquées dans le contrôle de la différenciation et la prolifération chondrocytaires. Afin de préciser les mécanismes moléculaires et cellulaires perturbés par les mutations de FGFR3, nous avons analysé le transcriptome de chondrocytes humains fœtaux à l'aide de puces Affymetrix U133-2. L'étude a porté sur une série de 8 cultures chondrocytaires primaires contrôles et 7 pathologiques, obtenues à partir de fœtus interrompus entre 18 et 25 semaines d'aménorrhée. Les chondrocytes pathologiques provenaient de fœtus atteints de nanisme thanatophore, portant les mutations activatrices gain de fonction Y373C, Y371C, R248C, $\mathrm{X} 807 \mathrm{R}$. Les échantillons contrôles ont été obtenus à partir de fœtus sans atteinte osseuse. Une normalisation GC-RMA et une correction des effets série a été réalisée à l'aide de la suite Partek. Les gènes présentant la plus forte variabilité d'expression ont été identifiés par la méthode d'Interquartile Range (IQR). Au total, 760 gènes ont été retenus et le rapport des niveaux d'expression moyens entre les deux groupes d'échantillons a été calculé. L'analyse fonctionnelle de ces gènes à l'aide d'Ingenuity Pathways Analysis est en accord avec les observations d'histologie et d'immunohistochimie montrant une diminution de la prolifération chondrocytaire dans la plaque de croissance pathologique. Elle confirme l'activation de la voie STAT et montre une perturbation de la synthèse de matrice extracellulaire, avec une réduction de l'expression de la plupart des collagènes et une surexpression des gènes de laminine et d'ostéopontine. De façon plus surprenante, nous avons observé une surexpression d'une centaine de gènes impliqués essentiellement dans l'adhésion et la motilité cellulaire. Ces premiers résultats semblent indiquer que la désorganisation de la structure de la plaque de croissance dans cette pathologie résulterait à la fois d'un défaut de synthèse de la matrice extracellulaire et de motilité des chondrocytes.

Mots-clés : FGFR3, achondroplasie, transcriptome.

EP408 / 400. FRÉQUENCE, SPECTRES PHÉNOTYPIQUE ET MUTATIONNEL DE SPG10 ET SPG31, DEUX NOUVELLES FORMES DE PARAPLÉGIE SPASTIQUE DOMINANTE AUTOSOMIQUE

C. Goizet (1), A. Durr (1, 2), E. Mundwiller (1), E. Ferdiko (2), S. Forlan (1), A. Toutain (3), P. Charles (2), D. Hannequin (4), B. Carlander (5), N. Carrière (6), M. Herbreteau (7), I. Le Ber (1), J.-F. Pinel (8), C. Tallaksen (1), J. Yaouanq (8), C. Depienne $(1,2)$, A. Brice $(1,2)$, G. Stevanin (1, 2)

(1) INSERM U679; (2) Département de Génétique et Cytogénétique, Hôpital de la Salpêtrière, 75651 Paris cedex 13 ; (3) Service de Génétique, CHU Tours ; (4) Service de Neurologie, CHU Rouen; (5) Service de Neurologie B, CHU Montpellier; (6) Service de Neurologie B, CHU Clermont-Ferrand; (7) Service de Neurologie, CH Lorient; (8) Services de Neurologie et de Génétique, CHU Rennes

Introduction : Les paraplégies spastiques héréditaires (PS) constituent un groupe hétérogène de maladies neurodégénératives se caractérisant par une spasticité des membres inférieurs de sévérité très variable, souvent associée à une faiblesse musculaire. L'absence ou la présence d'autres signes cliniques distingue les formes pures des formes complexes de PS. L'hétérogénéité génétique est très importante puisque 38 loci ont déjà été identifiés. Dans les formes de transmission autosomique dominante (AD), les muta-
SPG10 et SPG31 sont deux formes de PS AD pure, récemment décrites. Matériel et Méthodes Nous avons recherché des mutations dans les gènes KIF5A (SPG10) et REEPI (SPG31) chez 193 cas index non apparentés par séquençage direct. L'ensemble de la région codante et des jonctions intron-exon a été étudié pour REEPI (7 exons) tandis que l'analyse s'est limitée au 11 premiers exons du gène KIF5A (qui comporte 50 exons), correspondant au domaine moteur où sont essentiellement retrouvées les mutations décrites. L'analyse du gène REEPI est actuellement complétée par la recherche de réarrangements en utilisant la technique MLPA (MRCHolland). Résultats Nous avons identifié 8 mutations dans KIF5A (SPG10) et 5 mutations dans REEPI (SPG31). À l'exception d'une délétion intragénique dans $R E E P I$, la plupart de ces mutations sont toutes des faux-sens touchant des acides aminés très conservés au cours de l'évolution ou bien affectent l'épissage. Cinq mutations (4 pour SPG10 et une pour SPG31) sont associées à des formes complexes de PS essentiellement en raison de la présence d'une neuropathie axonale ou d'un retard mental. Discussion Nos résultats révèlent que SPG10 et SPG31 représentent des formes rares de PS $\mathrm{AD}, 2$ et $1 \%$ respectivement. L'âge de début est variable, de l'enfance à un âge avancé. Si la PS apparaît pure dans la majorité des cas, l'observation d'une forme complexe est possible aussi bien pour SPG10 que pour SPG31. Les mutations sont réparties sur toute la longueur du gène REEPI. Au contraire, un point chaud de mutations est observé dans les exons 8 à 10 du gène KIF5A, particulièrement au niveau du codon R280 (3 mutations faux-sens différentes identifiées). La recherche de réarrangements du gène REEPI, actuellement en cours, pourrait révéler une fréquence plus élevée des mutations de ce gène.

Mots-clés : paraplégies spastiques héréditaires, SPG10, SPG31.

EP409 / 402. ÉVALUATION DE L'IMPLICATION DES DIFFÉRENTS GËNES DU DESMOSOME ET D'AUTRES GÈNES CANDIDATS DANS LA DYSPLASIE ARYTHMOGÈNE VENTRICULAIRE DROITE

V. Fressart (1), F. Simon (1), E. Delacretaz (2), F. Hidden Lucet (1), C. Coirault (3), D. Keller (4), P. Cosnay (5), D. Klug (6), O. Cesari (7), R. Frank (1), B. Hainque (1) P. Charron (1)

(1) Hôpital Pitie-Salpêtrière, Paris, France ; (2) Hôpital de Berne, Switzerland; (3) Hôpital Bicêtre, Paris, France; (4) Hôpital de Bale, Bale, Switzerland; ; (5) Hôpital Bretonneau de Tours, France ; (6) Hôpital cardiologique de Lille, France; (7) Hôpital de Rennes, France

Introduction : La cardiomyopathie (ou dysplasie) arythmogène du ventricule droit (CVDA) est une maladie rare caractérisée par le remplacement du tissu myocardiaque par du tissu fibro adipeux prédominant dans le ventricule droit. C'est une maladie familiale dans $30 \%$ des cas à transmission classiquement autosomique dominante, causée par des mutations dans les gènes codant les protéines du desmosome. Des études préliminaires montrent que les mutations dans la plakophiline2 (PKP2) sont impliquées dans 11 à $47 \%$, dans la desmogleine2 (DSG2) dans $10 \%$ des cas. Les mutations dans la Desmoplakine $(D S P)$ représentent $5-16 \%$ des mutations causales. Les rares mutations (toutes récessives) dans le gène de la Plakoglobine (JUP) ont toutes été rapportées dans la maladie de Naxos (forme rarissime d'atteinte cardiaque et des phanères). But de l'étude : Notre but est d'identifier la fréquence des mutations dans les gènes du desmosome dans une population de patients indépendants présentant une CVDA et d'analyser cette fréquence selon le contexte familial et la sévérité. Notre but était également de tester de nouveaux gènes candidats, Desmine et SCN5A. Méthodes: Nous avons analysé chez 57 patients atteints de forme classique de CVDA par séquençage direct, après extraction de l'ADN, les gènes $P K P 2, D S G 2, D S C 2$, JUP et DSP. Nous avons analysé $S C N 5 A$ chez 17 patients, dont 10 chez qui la recherche de mutation sur les gènes du desmosome s'est avérée négative. L'analyse du gène codant le Desmine a été réalisée sur 11 patients dont la recherche de mutation dans les gènes du desmosme s'était révélée négative. Résultats: Nous avons identifié : 16 mutations dans $P K P 2$ (5 frame shift, 5 délétions, 3 insertions, 2 faux sens) et 3 variants de signification incertaine chez 18 des 57 patients index ; 6 mutations dans DSG2 (une mutation d'épissage, une délétion et une insertion ainsi que 3 mutations faux sens) chez 8 des 57 patients ; 3 mutations dans DSP ( 2 mutations faux sens et une mutation d'épissage) chez 3 patients ; 2 mutations dans DSC2 chez un même patient (une délétion, un variant faux sens); aucune mutation dans JUP. La recherche de mutation dans $S C N 5 A$ et dans la Desmine s'est avérée négative. Conclusions: Nous avons trouvé une fréquence élevée de mutations dans $P K P 2$ et $D S G 2$ parmi les patients atteints de DVDA (respectivement de $32 \%$ et $14 \%$ ). Plus rarement, nous avons mis en évidence des mutations dans le gène de la desmoplakine $(5 \%)$ et dans DSC2 $(2 \%)$. Ces résultats préliminaires montrent que les mutations causales de CVDA sont identifiées dans $50 \%$ des cas dans notre population étudiée, quelque soit 
le contexte (forme familiale ou sporadique). Ceci suggère l'utilité d'un génotypage des patients en routine de façon à améliorer la stratégie diagnostique et le conseil génétique des patients et des apparentés

Mots-clés : CVDA, diagnostic génétique, arythmie ventriculaire.

-1P410 / 409. DÉFICIT ANTEHYPOPHYSAIRE MULTIPLE ASSOCIÉ À UNE SURDITÉ, UN NOUVEAU SYNDROME EN RAP. PORT AVEC DES MUTATIONS DU FACTEUR DE TRANSCRIP. TION $L H X 3$

M.-L. Sobrier (1), C. Heinrichs (2), M.-P. Luton (1), S. Rose (1), S. Amselem (1)

(1) Inserm U654, Hôpital Trousseau, Paris ; (2) Unité d'Endocrinologie Pédiatrique, Hôpital Universitaire des Enfants Reine-Fabiola, Bruxelles

Introduction : $L H X 3$ code un facteur de transcription à domaine LIM jouant un rôle primordial pour le développement pituitaire et la spécification des motoneurones. Seulement 7 mutations de LHX3 ont été rapportées chez l'homme. Toutes ont été trouvées à l'état homozygote chez des patients consanguins présentant un déficit pituitaire combiné (CPHD, déficit en GH, TSH, PRL, FSH/LH, et parfois ACTH) ;à l'exception d'un cas, une rigidité cervicale est retrouvée chez tous ces patients, un trait phénotypique résultant probablement du défaut d'expression de $L H X 3$ au niveau des motoneurones. Résultats: Nous décrivons deux nouvelles mutations du gène $L H X 3$ à l'état hétérozygote composite identifiées chez un patient présentant non seulement un CPHD et une rigidité cervicale, mais également une surdité de perception profonde. Une mutation (c.252-3 C>G) affecte le site accepteur d'épissage de l'exon 3, l'autre, faux-sens (p.C123Y), implique un acide aminé très conservé du domaine LIM2. Dans le but d'étudier l'effet de la mutation c.252-3C > G sur l'épissage des transcrits $L H X 3$, des cellules HEK293 ont été transfectées avec des vecteurs d'expression contenant le minigène $L H X 3$ (s'étendant de l'exon $1 \mathrm{~b}$ à l'exon 5) normal ou muté. Cette mutation est responsable d'un épissage anormal, conduisant à la synthèse d'une protéine extrêmement tronquée. L'étude du retentissement fonctionnel de la mutation faux-sens (p.C123Y), par des tests luciférase sur différents promoteurs pituitaires, montre une perte partielle d'activation transcriptionelle de la protéine LHX3 mutée. Discussion: Ces données démontrent le caractère pathogène de ces deux nouvelles variations moléculaires de $L H X 3$. La surdité décrite chez ce patient non consanguin, nous incite à étudier le rôle possible de LHX3 dans le développement du système auditif chez l'homme.

Mots-clés : déficit pituitaire multiple, $L H X 3$, syndrome.

صP411 / 411. DÉFICIT GH ISOLÉ ET MALFORMATION DE CHIARI I CHEZ UN PATIENT PRÉSENTANT UNE NOUVELLE MUTATION D'ÉPISSAGE DU GHRHR

L. Hilal (1), Y. Hajaji (1), M.-P. Luton (2), Z. Ajaltouni (1), B. Benazzouz (1), M. Chana (3), A. Chraïbi (3), A. Kadiri (3), S. Amselem (2), M.-L. Sobrier (2)

(1) Laboratoire de Génétique et de Physiologie Endocriniennne, Faculté des Sciences, Kenitra, Maroc; (2) Inserm U654, Hopoital Trousseau, Paris; (3) Service d'Endocrinologie, CHU Ibn Sina, Rabat, Maroc

Introduction : Le déficit isolé en hormone de croissance (IGHD) peut être d'origine génétique. Un des rares gènes impliqués dans cette pathologie est celui codant pour le récepteur du GHRH (Growth Hormone Releasing Hormone). Résultats: Deux enfants marocains issus d'une union consanguine présentent un retard de croissance sévère (>-5SD) et une hypoplasie antéhypophysaire. En plus de ce phénotype classique de l'IGHD, un des patients a une malformation de Chiari I, un kyste arachnoïdien et une antéhypophyse de morphologie atypique. Aucune anomalie n'a été trouvée dans le gène $G H-N$. En revanche, le séquençage du gène GHRHR (exons et régions introniques flanquantes) a conduit à l'identification, chez les deux patients, d'une variation homozygote au site consensus d'épissage de l'intron 1 (c.57+2T>G). Par des expériences de transcription in vitro, nous montrons que cette mutation entraîne l'épissage anormal des transcrits du GHRHR, pouvant conduire à la synthèse d'une protéine sévèrement tronquée, délétée des domaines transmembranaires et intracellulaires. Discussion : Les anomalies de développement décrites pour un des patients, suggèrent un rôle possible du GHRHR dans le développement des structures extrapituitaires, par un mécanisme à élucider qui pourrait être direct ou secondaire au déficit sévère en $\mathrm{GH}$.

Mots-clés : mutation GHRHR, malformation Chiari I, épissage.

aP412 / 413. PHÉNOCOPIES DE LA MALADIE DE HUNTINGTON : INTÉRÊT DE L'EXAMEN NEUROPSYCHOLOGIQUE L. Freeman (1), V. Hahn-Barma (2), C. Cazeneuve (1), O. Russaouen (1), S. Humbert (3), P. Charles (1, 2), C.-M. Vincitorio (1), A. Brice $(1,2,4)$, A. Dürr $(1,4)$
(I) AP-HP, Hôpital de la Pitié-Salpêtrière, Département de Génétique et Cytogénétique, Paris, France; (2) AP-HP, Hôpital de la Pitié-Salpêtrière, Fédération des Maladies du Système Nerveux, Paris, France ; (3) Institut Curie, CNRS UMR 146, Orsay, France ; (4) INSERM U679, Paris, France

La maladie de Huntington est une affection de transmission autosomique dominante dont les manifestations cliniques sont dominées par l'existence de mouvements anormaux de type choréique, de troubles du comportement et d'une détérioration cognitive. Dans $90 \%$ des cas, elle est causée par une expansion anormale de triplets CAG dans le gène $I T I 5 / H D I$ situé en 4p16. Cependant, une hétérogénéité génétique existe : HDL1 (PrP), HDL2 (JPH3), SCA17 (TBP) et DRPLA (atrophine). La neuro-acanthocytose de transmission autosomique récessive et certaines affections acquises, comme le neuro-lupus, le syndrome des anti-phospholipides et certains cas de dysthyroidie auto-immune, ont été décrites comme pouvant donner des manifestations identiques. Huit cas index cliniquement diagnostiqués comme ayant une maladie de Huntington par des neurologues et neurogénéticiens spécialisés et chez qui la mutation classique n'a pas été retrouvée ont été sélectionnés. Ils présentaient un tableau clinique associant chorée,et troubles cognitifs d'évolution chronique. Certains présentaient des troubles du caractère et du comportement $(n=5)$, une dystonie $(n=4)$, un syndrome cérébelleux $(n=1)$, un syndrome pseudo-bulbaire $(n=1)$, des troubles sensitifs péri-buccaux, avec dysgueusie et perte de l'odorat $(n=1)$, une abolition des ROT avec amyotrophie distale des membres inférieurs $(n=1)$. Une histoire compatible chez des apparentés au premier degré a été retrouvée chez 3 patients sur 8. L'âge de début des symptômes était de 48 ans \pm 20 ans. Une expansion de triplet dans les gènes JPH3, TBP et DRPLA a été exclue. Un bilan auto-immun comprenant au moins : facteurs anti-nucléaires, anti-DNA natifs, anticardiolipines, ainsi qu'un bilan thyroïdien avec dosage des anticorps anti-thyroïdiens et un frottis sanguin à la recherche d'acanthocytes. Nous avons identifié un patient avec un diagnostic de syndrome primaire des antiphospholipides, amélioré considérablement sous traitement. Des tests neuropsychologiques ont été réalisés chez 6 des 7 cas index restant. Trois patients présentaient un syndrome dysexécutif avec d'importants troubles du maintien attentionnel et de la mémoire de travail correspondant au tableau habituellement décrit dans la maladie de Huntington. Chez 2 patients, le syndrome sous-cortico-frontal semble plus sévère que celui habituellement rencontré dans cette pathologie. Chez une patiente, il a été retrouvé seulement un discret déficit attentionnel, sans éléments de dysfonction exécutive. La réalisation de tests neuropsychologiques a permis d'identifier un sous-groupe de trois patients présentant un profil typique de maladie de Huntington qui correspond à une phénocopie véritable sans la mutation par expansion CAG dans le gène $\mathrm{HD} 1$. Le séquençage complet du gène HD1 est en cours.

Mots-clés: maladie de Huntington, phénocopies, examen neuropsychologique.

- 9413 / 415. NOUVELLES MUTATIONS DE NOVO DE SOX2 CHEZ DES PATIENTS PRÉSENTANT UNE ATTEINTE OPHTALMIQUE SÉVÈRE ASSOCIÉE À D'AUTRES ANOMALIES CÉRÉBRALES DU DÉVELOPPEMENT

M.-P. Vié-Luton (1), M.-L. Sobrier (1), C. de Barace (2), E. Feigerlova (3), S. Rose (1), N. Idres (2), S. Odent (4), M. Tauber (3), S. Amselem (1)

(I) Inserm U654, Hôpital Trousseau, Paris ; (2) Unité Pédiatrique, $\mathrm{CH}$ Saint-Brieuc; (3) Hôpital d'Enfants, Toulouse ; (4) Génétique Médicale, CHU Rennes

Introduction : SOX2 est un facteur de transcription impliqué dans la régulation du développement et de la détermination cellulaire. Les souris hétérozygotes pour l'invalidation de Sox 2 présentent un développement antéhypophysaire anormal avec des taux de GH, LH et TSH diminués, sans aucun défaut oculaire associé. Chez l' homme, les quelques mutations SOX2 hétérozygotes identifiées peuvent provoquer une anophtalmie/microphtalmie bilatérale et/ou un retard de développement, des anomalies du corps calleux, une atrésie œsophagienne, une surdité d'origine neuro-sensorielle. Résultats : Nous avons étudié deux patients indépendants présentant de sévères anomalies oculaires ; le premier a une anophtalmie droite et une hypoplasie du nerf optique gauche ; le second, issu d'une union consanguine, présente une anophtalmie bilatérale ainsi qu'un hypogonadisme hypogonadotrope et une petite taille. L'IRM du premier patient montre un corps calleux mince et, celle du deuxième un kyste du septum-pellucidum. Ces deux patients sont porteurs de nouvelles mutations de novo de SOX2 à l'état hétérozygote : le patient \#1 est porteur d'une mutation faux-sens (p.W51R) impliquant un résidu très conservé au cours de l'évolution situé dans le domaine HMG de la protéine. Une délétion d'une base (c.540delC) a été identifiée chez le patient \#2 entraînant un décalage du cadre de lecture de la protéine SOX2:21 nouveaux acides aminés sont insérés en amont 
d'un codon STOP prématuré à la position 202 , conduisant à une protéine tronquée. Discussion: Des études complémentaires sont en cours afin d'évaluer les conséquences fonctionnelles de ces deux nouveaux défauts de SOX2.

Mots-clés : $S O X 2$, mutations, développement.

IP414 / 420. DES MUTATIONS DU GENE OPA1 SONT RESPONSABLES DE FORMES SYNDROMIQUES D'ATROPHIE OPTIQUE ASSOCIÉES À DES DÉLÉTIONS MULTIPLES DE L'ADN MITOCHONDRIAI

P. Amati-Bonneau (1, 2), P. Labauge (3), P. Marcorelles (4), D. FigarellaBranger (5), A. Furby (6), F. Letournel (7), B. Wissinger (8), C. Verny (9), C. Ayuso (10), R. Garesse (11), G. Lenaers (12), V. Carelli (13), P. Reynier (1, 2), D. Bonneau $(1,2)$

(1) Département de Biochimie et Génétique, Centre Hospitalier Universitaire d'Angers ; (2) INSERM U694, Angers, France ; (3) Service de Neurologie, Centre Hospitalier Universitaire, Nîmes, France; (4) Service d'Anatomie Pathologique, Centre Hospitalier Universitaire, Brest, France (5)Service d'Anatomie Pathologique et Neuropathologie, Centre Hospitalier Universitaire, Hôpital de la Timone, Marseille, France; (6) Service de Neurologie, Centre Hospitalier de Saint-Brieuc, Saint-Brieuc, France; (7) Laboratoire de Neurobiologie et Neuropathologie, Centre Hospitalier Universitaire d'Angers, Angers, France ; (8) Molecular Genetics Laboratory, University Eye Hospital Tuebingen, Germany; (9) Département de Neurologie, Centre Hospitalier Universitaire d'Angers, Angers, France; (10) Departamento de Bioquimica Instituto de Investigaciones Biomedicas Alberto Sols ${ }^{\prime}$ CSIC-UAM, Facultad de Medicina, Universidad Autonome de Madrid, CIBERER, ISCIII,Madrid, Spain; (11) Centro de Investigación, and Servicio de Neurología, Hospital Universitario 12 de Octubre, CIBERER, ISCIII, Madrid, Spain; (12) INSERM U583, Institut des Neurosciences de Montpellier, Universités de Montpellier I et II, Montpellier. France; (13) Dipartimento di Scienze Neurologiche, Università di Bologna, Bologna, Italy

La protéine OPAl est une dynamine-GTPase codée par un gène nucléaire et localisée dans la membrane interne de la mitochondrie. Elle est impliquée dans le processus de fusion des mitochondries, l'organisation des crêtes mitochondriales et le contrôle de l'apoptose. Les mutations du gène $O P A I$ sont responsables de 60 à $70 \%$ des atrophies optiques autosomiques dominantes (AOD). Il s'agit, en général, de mutation tronquantes responsables d'atrophies optiques isolées. Nous rapportons ici des mutations du gène $O P A l$ chez des patients ayant une atrophie optique associée à d'autres signes neurologiques et à une atteinte musculaire avec délétions multiples de l'ADN mitochondrial (ADNmt) suggèrant qu'OPAl est également impliqué dans la maintenance de l'ADNmt. Patients, méthodes et résultats : Huit patients appartenant à six familles ont été étudiés. Les signes cliniques étaient les suivants: atrophie optique $(8 / 8)$, ophtalmoplégie externe $(2 / 8)$, Ptôsis $(2 / 8)$, surdité neurosensorielle $(7 / 8)$, ataxie $(3 / 8)$, signes musculaires cliniques (2/8) et neuropathie axonale (4/6 testés). Des mutations faux sens du gène $O P A l$ ont été mises en évidence à l'état hétérozygote chez tous les patients. Toutes ces mutations entrainaient une substitution d'un acide aminé très conservé dans le domaine GTPase d'OPAI et n'ont pas été retrouvées sur 460 chromosomes contrôles. Une mutation était de novo, les cinq autres étaient héritées. Les biopsies musculaires ont montré la présence de ragged red fibers (RRF) et de fibres Cytochrome c Oxidase (Cox) negatives chez 5 des 6 patients. Le dernier patient présentait des signes de myopathie non spécifique. Des délétions multiples de l'ADNmt ont été identifiées dans le muscle de tous les patients par Southern-blot et/ou par LINK Word.Document.8 C :I WRL2564.tmp.doc OLE_LINK2 la \r PCR longues de l'ADNmt. Le réseau mitochondrial était fragmenté sur les fibroblastes de 4 des patients. Le séquençage des gènes classiquement impliqués dans les délétions multiples de l'ADNmt (POLG1, Twinkle et ANT1) s'est avéré normal. Discussion : Nous montrons que des mutations faux sens du gène $O P A l$ sont associées à une instabilité de l'ADNmt et entraînent des phénotypes cliniques complexes pour lesquels nous proposons le terme d'OPA $I$ « plus ». Ces phénotypes comprennent de façon constante une atrophie optique et atteinte musculaire allant de formes non spécifiques de myopathie jusqu'a des myopathies mitochondriales classiques avec ophtalmoplégie externe, RRF et fibres Cox négatives à l'histologie. À ces signes, sont souvent associées une surdité neurosensorielle, une ataxie cérébelleuse et une neuropathie périphérique axonale. L'anomalie moléculaire la plus marquante est la présence constante de délétions multiples de l'ADNmt dans le muscle. Ces données suggèrent un nouveau rôle pour $O P A I$ dans le maintien de l'intégrité de l'ADNmt faisant de ce gène le cinquième gène responsable de délétions multiples de l'ADNmt après ceux codant pour les et ANT1. Le mécanisme par lequel $O P A I$ intervient dans la maintenance de l'ADNmt est spéculatif. Le fait que l'on observe chez tous nos patients des mutations faux-sens écarte un mécanisme d'haplo-insuffisance souvent impliqué dans les mutations d'OPAI responsables d'atrophie optique isolée. OPA1 est une protéine de la membrane interne de la mitochondrie ayant un rôle crucial dans la conformation des crêtes et du réseau des mitochondries. Il est tentant de spéculer que la désorganisation des crêtes et la fragmentation du réseau mitochondrial (observé sur les fibroblastes de quatre de nos patients) pourraient interagir avec les nucleoïdes qui sont les organelles contenant l'ADNmt. En conclusion, nous rapportons l'implication d'OPAl dans la maintenance de l'ADNmt et dans des manifestations cliniques similaires à ce que l'on observe dans les encéphalo-myopathies mitochondriales classiques.

Mots-clés : mitochondrie, délétion multiple, OPA1.

- 415 / 423. ÉTUDE DU GÈNE SFI CHEZ 25 FILLES ATTEINTES D'AMÉNORRHÉE PRIMAIRE À CARYOTYPE NORMAL

B. Lakhal (1), R. Braham (2), P. Philipert (3), M. Chaieb (2), S. Hidar (4), M. Zaouali (5), C. Sultan (3), A. Saad (1), H. Elghezal (1)

(1) Service de Cytogénétique et de Biologie de Reproduction, Hôpital Farhat-Hached, Sousse, Tunisie ; (2) Service d'Endocrinologie, Hôpital Farhat-Hached, Sousse, Tunisie; (3) Service d'Hormonologie du Développement et de la Reproduction, Hôpital Lapeyronie, Montpellier. France ; (4) Service de Gynécologie Obstétrique, Hôpital Farhat-Hached, Sousse, Tunisie : (5) Service de Physiologie et des Explorations Fonctionnelles, Faculté de Médecine de Sousse, Tunisie

Introduction : SF1 (Steroidogenic factor 1) est un récepteur nucléaire qui régule plusieurs gènes impliqués dans le développement et le fonctionnement des gonades. Des mutations du gène $S F 1$ ont été retrouvées chez des femmes $X Y$ atteintes d'insuffisance gonadique et surrénalienne. Des mutations de ce gène à l'état hétérozygotes sont récemment retrouvées associées à des dysgénésies gonadiques partielle ou totale, sans insuffisance surrénalienne, témoignant de l'hétérogénéité clinique des mutations touchant ce gène. L'objectif de ce travail est de rechercher des éventuelles mutations du gène SFI chez les femmes atteintes aménorrhée primaire en dehors du syndrome de Turner. Matériel et méthodes: 25 aménorrhées primaires, sans auto-immunité personnelle et à caryotype normal, sont explorées par la technique de séquençage direct de la partie codante du gène $S F 1$. Un groupe de 50 femmes témoins a été également étudié par séquençage de I'exon 4 du gène $S F 1$. Résultats : $48 \%$ des patientes présentent le variant G146A au niveau de l'exon 4 du gène $S F 1$. Chez le groupe témoin, ce variant est retrouvé seulement chez $4 \%$ des femmes. Discussion et conclusion Le variant G146A a été retrouvé chez un garçon atteint d'un hypogonadisme périphérique mais non encore décrit associé aux insuffisances ovariennes prématurées. Deux études ont montré que G146A réduit de $20 \%$ la fonction de transactivation de SF1. Notre étude montre que le retentissement phénotypique de ce varient touche aussi les filles et se traduit par un tableau d'insuffisance ovarienne prématurée avec aménorrhée primaire.

Mots-clés : SF1, aménorrhée primaire, variant G146A.

EP416 / 425. MUTATIONS DES GENNES HADHA ET HADHB : L'EXPERIENCE FRANÇAISE À PARTIR D'UNE COHORTE DE 46 PATIENTS

M. Brivet (1), C. Acquaviva (2), A. Boutron (1), C. Vianey-Saban (2), M.T. Zabot (3), A. Legrand (1)

(1) Laboratoire de Biochimie, AP-HP hôpital de Bicêtre, Le KremlinBicêtre; (2) Service des Maladies Métaboliques; (3) Centre de Biotechnologie Cellulaire, Centre de Biologie Est, Lyon

Introduction : Les gènes $H A D H A$ et $H A D H B$ codent les deux sous-unités d'une protéine mitochondriale impliquée dans trois réactions enzymatiques successives de l'oxydation des acides gras à longue chaîne. La sous-unité alpha (HADHA) porte une fonction hydratase et une fonction deshydrogénase (3-hydroxy AcylCoA deshydrogénase ou LCHAD) et la sous-unité béta $(H A D H B)$ une fonction thiolase. Les mutations de HADHA ou $H A D H B$ se transmettent sur le mode autosomique récessif, entraînant chez le sujet homozygote des anomalies sévères et précoces du métabolisme énergétique, avec une intolérance au jeûne prolongé et un dysfonctionnement cardiaque et musculaire. Certaines mutations sont aussi responsables d'une stéatose aigüe gravidique chez les femmes enceintes hétérozygotes porteuses d'un fœtus atteint. Méthodes : L'orientation initiale du diagnostic est biochimique : elle se fait par la mise en évidence de métabolites anormaux qui s'accumulent en amont du bloc enzymatique (acides organiques urinaires ou acylcarnitines sanguines). Le diagnostic enzymatique direct n'est plus réalisable car les réactifs nécessaires ne sont plus 
commercialisés. La recherche de mutations reste donc la seule solution actuelle pour confirmer le diagnostic. Notre stratégie de recherche des mutations est la suivante : nous recherchons d'abord la mutation fréquente du gène $H A D H A$ à partir de l'ADN génomique, puis nous complétons l'étude par le séquençage direct des 2 ADNc $H A D H A$ et $H A D H B$ obtenus par RT-PCR à partir de l'ARN extrait d'une culture de fibroblastes prétraitée par l'émétine. Nous confirmons la présence des mutations ainsi identifiées sur l'ADN génomique du cas index et de ses parents. Le séquençage de l'ADN génomique du cas index en première intention (20 exons pour $H A D H A$ et 16 exons pour $H A D H B$ ) n'est réalisé que si aucun matériel cellulaire ne peut être mis en culture. Patients : La cohorte de patients que nous présentons représente l'ensemble des patients diagnostiqués en France depuis la première description biochimique du déficit en LCHAD en 1989. Résultats et discussion: La mutation fréquente c. $1528 \mathrm{G}>\mathrm{C}$ du gène $H A D H A$ a été retrouvée sur $53 \%$ des allèles étudiés (49/92), 18 fois à l'état homozygote, 13 fois à l'état hétérozygote. Seize autres mutations du gène $H A D H A$ ont été identifiées (dont 12 nouvelles) et 7 mutations du gène $H A D H B$ (dont 6 nouvelles) : mutations faux sens, non-sens, insertions ou délétions de quelques nucléotides, anomalies d'épissage et une grande délétion hétérozygote de $5.9 \mathrm{~Kb}$ éliminant les exons 2 et 3 du gène $H A D H B$ par recombinaison homologue entre 2 séquences Alu. Les mutations des 2 allèles ont été identifiées chez tous les patients étudiés. Il est à noter que 12 mutations sur 23 provoquaient l'apparition directe ou indirecte d'un codon-stop susceptible d'induire la dégradation de l'ARN messager par le phénomène de nonsense mediated mRNA decay (NMD) ; grâce au prétraitement du matériel cellulaire par un inhibiteur de la traduction (émétine) avant d'extraire l'ARN, nous avons néanmoins pu mettre en évidence l'ensemble de ces mutations au niveau de l'ADNc. Conclusion : Le séquençage de l'ADNc n'avait pas encore été utilisé de manière systématique pour rechercher les mutations des gènes HADHA et HADHB. Il nous a permis d'identifier les mutations sur $100 \%$ des allèles étudiés à la différence des séries précédentes de la littérature, explorées à partir de l'ADN génomique. Nous rapportons en particulier pour la première fois, l'existence d'une grande délétion hétérozygote du gène $H A D H B$, qui serait passée inaperçue au séquençage de l'ADN génomique exon par exon.

Mots-clés : nonsense mRNA mediated decay (NMD), émetine, gènes $H A D H A$ et $H A D H B$.

E417 / 431. ÉTUDE GÉNÉTIQUE DE 16 FAMILLES AVEC ÉPILEPSIE AUTOSOMIQUE DOMINANTE DU LOBE TEMPORAL LATÉRAL

E. Chabrol (1), I. Gourfinkel-An (1, 2), P. Couarch (1), O. Trouillard (3),

T. Sanchis (4), M. Baulac (2), E. Le Guern (1, 3), S. Baulac (1)

(1) INSERM, UMR 679, Neurologie \& Thérapeutique Expérimentale, Université Pierre et Marie Curie-Paris 6, Faculté de Médecine, Hôpital de la Pitié-Salpêtrière, Paris; (2) Unité d'Épileptologie, AP-HP, Hôpital de la Pitié-Salpêtrière, Paris ; (3) Laboratoire de Neurogénétique, Departement de Génétique, Cytogénétique et Embryologie, AP-HP, Université Pierre-etMarie-Curie, Paris 6, Faculté de Médecine, Hôpital de la Pitié-Salpêtrière, Paris ; (4) UIB de Génétique, Centre d'Investigations Biologiques (CIB), GH Pitié-Salpêtrière, DRC, AP-HP, Paris

L'épilepsie autosomique dominante du lobe temporal (ADLTE pour autosomal dominant lateral temporal epilepsy) est une épilepsie idiopathique qui débute dans l'adolescence et qui est caractérisée par des crises focales avec des auras auditives (signes auditifs déclencheurs ou hallucinations auditives lors des crises) et/ou des symptômes évoquant un foyer temporal latéral des crises. En 2002, le gène LGII/Epitempine (Leucine-rich, glioma inactivated 1) a été impliqué dans cette épilepsie. À ce jour les mutations dans ce gène sont identifiées chez $\sim 30 \%$ des familles ADLTE; ce qui suggère une hétérogénéité génétique. L'identification de mutations nonsens, de mutations modifiant le cadre de lecture de LGII et de mutations faux sens conduisant à une perte de fonction de la protéine mutée est compatible avec un mécanisme d'haplo-insuffisance. Récemment, le récepteur de LGI1, ADAM22 (A Dinsintegrin And Metalloproteinase Domain 22), une protéine transmembranaire, a été identifié. La liaison de LGIl à ADAM 22 entraîne une potentialisation de la transmission glutamatergique médiée par les récepteurs AMPA. Il a été également démontré que LGI1 se lie à ADAM23, une autre protéine transmembranaire de la famille ADAM. L'objectif de notre étude est de rechercher le gène responsable du syndrome épileptique dans 16 familles avec ADLTE. Nous avons dans un premier temps recherché les mutations dans le gène $L G I I$ et avons identifié deux nouvelles mutations, p.Leu232Pro et $\mathrm{c} .431+1 \mathrm{G}>\mathrm{A}$ chez deux d'entre elles. Les études fonctionnelles ont permis de démontrer l'implication des mutations dans le phénotype et déterminer leurs conséquences : une perte de fonction des protéines mutantes. Nous avons aussi exclu la présence d'un réarrangement du gène LGII (délétions ou duplications exoniques) par dosage génique en Q-PCR et celle d'une mutation dans la région promotrice du gène. Dans les familles atteintes d'ADLTE pour lesquelles aucune mutation de LGI1 n'avait été identifiée. nous avons ensuite séquencé les gènes $A D A M 22$ et $A D A M 23$ par séquençage direct des parties codantes et flanquant les exons et avons montré que ce ne sont pas des gènes majeurs pour les ADLTE.

Mots-clés : LGII, épilepsie, ADAM22.

- 418 / 432. DYSGÉNÉSIE TUBULAIRE RÉNALE AUTOSOMIQUE RÉCESSIVE ET MUTATIONS DES GÈNES DU SYSTÈME RÉNINE-ANGIOTENSINE

O. Gribouval (1), V. Morinière $(1,2)$, A. Pawtowski $(1,2)$, C. Loirat (3), J. Bachetta (4), P. Cochat (4), F. Dijoud (5), R. Bouvier (6), G. Viot (7), J. Tantau (8), T. Neuhaus (9), J. Aziza (10), P. Corvol (11), J.-M. Gasc (11), C. Antignac (1, 2), M.-C. Gubler (1)

(1) Inserm U574, Hôpital Necker-Enfants Malades, Paris ; (2) Service de Génétique, Hôpital Necker-Enfants Malades, Paris; (3) Service de Néphrologie Pédiatrique, Hôpital Robert-Debré, Paris; (4) Service de Néphrologie Pédiatrique, Hôpital Édouard-Herriot, Lyon ; (5) Laboratoire A'Anatomie Pathologique, Hôpital Édouard-Herriot, Lyon; (6) Laboratoire đ'Anatomie Pathologique, Hôpital Debrousse, Lyon ; (7) Service de Génétique Médicale, Hôpital St-Vincent-de-Paul, Paris ; (8) Service d'Anatomie Pathologique, Hôpital St-Vincent-de-Paul, Paris; (9) University Children's Hospital, CH-8032 Zurich; (10) Laboratoire d'Anatomie Pathologique, Hôpital Purpan, Toulouse ; (11) Inserm U833, Collège de France, Paris

La dysgénésie tubulaire rénale (DTR) autosomique récessive est une anomalie sévère du développement rénal. Elle se manifeste par une anurie fœtale persistante responsable d'oligoamnios et le plus souvent, de mort périnatale. Il s'y associe une hypotension réfractaire observée chez les nouveau-nés survivants et un retard d'ossification de la voûte crânienne. La néphropathie est caractérisée histologiquement par l'absence ou la présence d'un nombre très réduit de tubes proximaux différenciés. Les reins sont normaux ou peu modifiés à l'échographie. Nous avons récemment montré, à partir de l'étude de patients appartenant à 9 familles, que cette néphropathie était génétiquement hétérogène et liée à des mutations des gènes $A G T, R E N, A C E$ et $A G T R I$ codant les différentes protéines du système rénine-angiotensine (SRA). Au cours des deux dernières années, 31 nouveaux patients (fœtus, nouveau-nés et jeunes enfants) appartenant à 19 familles nous ont été adressés pour confirmation du diagnostic et étude moléculaire. Globalement, 15 des 28 familles étudiées étaient consanguines et des mutations ont été identifiées dans 26 d'entre elles, permettant de réaliser un diagnostic prénatal dans 3 familles. Des mutations du gène REN étaient présentes dans 8 familles à l'état homozygote ( 6 familles) ou hétérozygote composite ( 2 familles) : mutations perte de fonction dans 5 cas, caractérisées par l'absence de production de rénine; mutations faux-sens dans 3 cas aboutissant à l'hyperproduction de la protéine mutée non fonctionnelle. Des mutations des gènes $A G T, A G T R I$ et $A C E$, ont été détectées dans respectivement 2, 2 et 14 familles. Elles étaient présentes à l'état homozygote dans 9 familles. Leur conséquence est l'absence de génération d'angiotensine II (mutations des gènes codant l'angiotensinogène ou l'enzyme de conversion de l'angiotensine) ou la production d'une angiotensine II inefficace (mutations du gène AGTRI codant le récepteur de type 1 de l'angiotensine II). Toutes étaient associées à une hyperexpression rénale de rénine témoignant de la perte de sa régulation négative par l'angiotensine II. Aucune mutation n'a été détectée dans 2 familles. L'atteinte de fotus de sexe féminin dans chacune de ces familles a permis d'exclure les gènes codant le récepteur de type 2 de l'angiotensine II ou le récepteur de la rénine, localisés sur le chromosome X. Quel que soit le gène muté, l'évolution de la maladie a presque toujours été défavorable : mort in utero, interruption médicale de grossesse du fait de la sévérité et de la précocité ou de la récidive de l'anamnios dans la fratrie, mort rapide post-natale liée à la persistance de l'anurie et à la détresse respiratoire, malgré la prise en charge thérapeutique. Parmi les 4 enfants survivants, un a été transplanté à l'âge de 4 ans, un autre, âgé de 2 ans est dialysé depuis la naissance. Une patiente âgée de 15 , ayant à l'état homozygote une mutation faux-sens de l'AGT, a une insuffisance chronique modérée. Un patient, âgé de 8 ans a une fonction rénale normale. Il est porteur de mutations hétérozygotes composites de l'ACE, l'une d'elles étant une mutation fauxsens. Aucune anomalie n'a été observée chez les parents porteurs de mutations à l'état hétérozygote. En conclusion, la DTR récessive autosomique est une cause significative d'anurie fœtale. Ce diagnostic doit être discuté devant toute anurie fœtale à reins normaux ou peu modifiés à l'examen échographique. Des mutations des gènes codant les différentes protéines du SRA sont mises en évidence dans la majorité des cas. Elles s'accompagnent constamment d'une dérégulation de l'expression rénale et 
systémique de la rénine. Contrairement à nos premiers résultats, les mutations de l'ACE semblent les plus fréquentes. Le phénotype est le même quels que soient le gène muté et l'expression de rénine, montrant qu'il n'existe pas de redondance in vivo dans le SRA.

Mots-clés : Mutations, Système rénine-angiotensine, Dysgénésie tubulaire rénale.

\section{- 1 P419 / 433. ANALYSE DÉTAILLÉE DE LA RÉGION 17P11.2 CHEZ 59 PATIENTS ATTEINTS DE SYNDROME DE SMITH-MAGENIS}

C. Bendavid (2), M. Huizing (1), H. Edwards (1), C. Ciccone (1), M.P. Jones (1), S.C. Chandrasekharappa (1), J. Blancato (3), W.A. Gahl (1), A.C.M. Smith (1)

(1) NHGRI/NIH, Bethesda, MD, USA ; (2) UMR 6061 CNRS, Université de Rennes-1, Rennes, France; (3) Georgetown University, Washington, $D C, U S A$

Le Syndrome de Smith Magenis (SMS) est caractérisé par des anomalies craniofaciales et squelettiques particulières, des retards de langage, de développement psychomoteur et de croissance, un phénotype comportemental avec des troubles chroniques du sommeil et un rythme circadien inversé de sécrétion de la mélatonine. La plupart des cas sont dus à une délétion interstitielle en 17p11.2. Cependant, de rares cas non délétés ont été rapportés à des mutations de RAII. Nous avons effectué une corrélation génotype-phénotype sur 59 patients SMS. Les études phénotypiques ont révélé des caractéristiques cliniques uniques ou inconstantes incluant: déficit auditif, faibles taux d'IgA, cholestérol élevé et anomalies squelettiques. Nous avons employé des marqueurs polymorphes de la région $17 \mathrm{p}$ pour déterminer l'origine parentale de l'allèle délété et mis en évidence plutôt une origine maternelle dans $63 \%$ des cas ce qui n'est pas statistiquement significatif. Des analyses par FISH et PCR quantitative en temps réel (qPCR) ont été réalisées pour déterminer le statut en nombre de copies de la région 17p11.2. Les analyses en qPCR pour six gènes d'intérêt de la région minimale critique du SMS ont été réalisées, incluant $R A I I$ et $R A S D I$ (impliqués dans le rythme circadien), MYO15A (impliqué dans des déficits auditifs), FLII (en relation avec la réponse immunitaire), PEMT (jouant un rôle dans le métabolisme des cholines) et TNFRSF13B (impliqué dans le déficit en $\operatorname{IgA}$ ). La majorité ( $56 \%$ ) des patients avait la délétion la plus commune de $17 \mathrm{p} 11.2$ couvrant $3,5 \mathrm{Mb}$. 11 patients $(19 \%)$ avaient une délétion de taille atypique mais sans différences concernant l'atteinte des 6 gènes d'intérêt. 15 patients $(25 \%)$ ne montraient pas de délétion par FISH ni qPCR, et le séquençage de $R A I I$ n'a à ce jour révélé qu'une mutation (P242L) parmi ce groupe. L'étude en CGH array (Agilent 44K) de ces patients non délétés a permis chez 4 patients d'identifier de possibles réarrangements chromosomiques responsables de "SMS like». Cette étude montre à nouveau la difficulté que l'on peut avoir à établir de fortes corrélations génotype-phénotype dans certaines pathologies génétiques. L'identification plus précise des points de cassure et des groupes de gènes dont le nombre de copies varie permettra peut être à l'avenir d'affiner nos connaissances sur les phénotypes associés.

Mots-clés : syndrome de Smith Magenis, syndrome délétionnel, cytogénétique moléculaire.

WP420 / 449. MUTATION M.10191T>C DE L'ADNMT RESPONSABLE D'UNE ENCÉPHALOPATHIE CHEZ 2 ENFANTS : SURVENUE DE NOVO?

S. Bannwarth (1), K. Fragaki (1), S. Mutz (1), V. Procaccio (2), B. Chabrol (3), D. Figarella-Branger (4), J.-C. Lambert (1), V. Paquis-Flucklinger (1) (1) Service de Génétique Médicale, Centre de Référence des maladies mitochondriales, Hôpital Archet 2, CHU Nice ; (2) Center for Molecular and Mitochondrial Medicine and Genetics, Université de Californie, Irvine, USA ; (3) Service de neuropédiatrie, Hôpital Timone, CHU Marseille: (4) Service d'anatomopathologie et neuropathologie, Hôpital Timone, CHU Marseille

Les déficits isolés en complexe I sont les défauts les plus fréquemment retrouvés dans les pathologies mitochondriales. Ils sont liés dans plus de $90 \%$ des cas à des mutations siégeant dans des gènes nucléaires. Nous décrivons 2 enfants présentant un déficit en complexe I secondaire à une mutation (m.10191T>C) dans le gène ND3 de l'ADNmt. Dans les 2 cas, il n'existe aucun antécédent familial. Le premier enfant est né à 31 SA par césarienne. La grossesse avait été marquée par un RCIU et un oligoamnios. Un retard des acquisitions est noté au cours des premiers mois. La marche est acquise à l'âge de 2 ans. Peu après, survient un épisode de déficit au niveau facial. L'IRM montre des lésions à type de gliose périventriculaire. Une régression apparaît à l'âge de 3 ans avec des difficultés à la marche ataxie, un syndrome pyramidal bilatéral et une hémiparésie droite. L'IRM met alors en évidence des lésions diffuses corticales avec une atteinte des noyaux gris et un pic de lactate en spectroscopie. Le second enfant est né à terme de parents non consanguins. À l'âge de 4 mois, il présente une hypotonie avec un retard des acquisitions et plusieurs épisodes convulsifs. L'IRM cérébrale est en faveur d'un syndrome de Leigh. Chez ces 2 enfants, nous avons retrouvé un déficit isolé en complexe I de la chaîne respiratoire à partir d'une biopsie musculaire. Une étude par Surveyor nous a permis d'identifier la mutation m.10191T >C, à l'état hétéroplasmique, dans le gène $N D 3$ de l'ADNmt. Cette mutation (p.S45P) entraîne le remplacement d'un acide aminé Sérine hydrophile par une Proline hydrophobe dans un domaine très conservé de la protéine. Elle est présente, dans le muscle et les leucocytes de ces 2 enfants, à un haut niveau d'hétéroplasmie. Dans les 2 familles, la mutation m.10191T $>C$ n'a pas été retrouvée à partir de l'ADN extrait des leucocytes chez les mères et chez des apparentés maternels. Ce résultat peut être en faveur d'une survenue de novo, qui a déjà été évoquée dans un petit nombre de cas rapportés dans la littérature. L'étude des ascendants maternels, sur d'autres types cellulaires, est en cours dans la première famille afin de tenter de conforter cette hypothèse. Néanmoins, la discussion insistera sur la nécessité de rester extrêmement prudent en termes de conseil génétique et de conduite à tenir en cas de future grossesse.

Mots-clés : ADNmt Déficit Complexe I Pathologie mitochondriale.

IP421 / 454. ANALYSES MUTATIONNELLE ET TRANSCRIPTIONNELLE DU GÈNE, IMPLIQUÉ DANS CMT4H, UNE FORME DÉMYÉLINISANTE DE CMT À TRANSMISSION AUTOSOMIQUE RÉCESSIVE

C. Baudot (1), Y. Poitelon (1), S. Stora (1), T. Hamadouche (1, 2), I. Boccaccio (1), M. Chaouch (3), N. Kassouri (3), R. Bernard (4), A. Megarbané (5), N. Lévy $(1,4)$, V. Delague (1)

(1) INSERM U491, "Génétique Médicale et Développement ", Université de la Méditerranée, Faculté de Médecine de la Timone, 13385 Marseille, France; (2) Laboratoire de Biologie Moléculaire, Institut Pasteur, Alger, Algérie; (3) Service de Neurologie, Centre Hospitalier Universitaire Ben Aknoun, Algers, Algeria ; (4) AP-HM, Département de Génétique Médicale, Hôpital d'enfants Timone, Marseille, France; (5) Unité de Génétique Médicale, Université Saint-Joseph, Beyrouth, Liban

Introduction : La maladie de Charcot-Marie-Tooth (CMT) constitue un groupe de maladies, hétérogène à la fois sur le plan clinique et génétique. Elles sont divisées en deux groupes : le CMT démyélinisant (CMT1) et le CMT axonal (CMT2). Elles se caractérisent par une faiblesse musculaire, des déformations des pieds et des mains et un abaissement des vitesses de conduction nerveuse. Delague et al. (2007), ont identifié un gène impliqué dans une forme démyélinisante autosomique récessive : CMT4H. Il s'agit du gène FGD4 sur le chromosome 12, qui code pour FGD4/FRABIN (FGD1-related F-Actin Binding protein). Deux mutations ont été identifiées dans deux familles consanguines d'origine Méditerranéenne: une mutation d'épissage c.893T>G (p.298MetfsX8) dans une famille libanaise et une mutation faux-sens c.893T >C (p.Met298Thr) dans une famille algérienne. Par ailleurs, trois autres mutations non-sens ou frame-shift ont été identifiées par une autre équipe (Stendel $e t$ al., 2007). Nous avons mené des études transcriptionnelles, à la fois chez des patients, des individus sains, ainsi que sur différents tissus humains. Par ailleurs, nous avons recherché des mutations dans la séquence codante de FGD4 dans une cohorte de 96 patients atteints de formes sévères de CMT démyélinisant à transmission autosomique récessive ou sporadiques, et pour lesquels aucune mutation dans les gènes connus n'a pu être identifiée Résultats Chez le patient libanais, nous avions caractérisé un transcrit anormal utilisant un site donneur d'épissage dans l'exon 7, dont l'utilisation est favorisée par la mutation C.893T>G, et qui entraîne la délétion de quatre des cinq domaines fonctionnels de la protéine FRABIN, en particulier le domaine $\mathrm{DH}$, responsable de l'activité RhoGEF. L'analyse transcriptionnelle chez des individus sains a permis de montrer l'existence de nombreux autres transcrits alternatifs, dont certains codent des protéines dépourvues de certains domaines fonctionnels. Par exemple, nous avons pu identifier un transcrit délété de l'exon 3, contenant le codon ATG initiateur, qui selon les prédictions, utiliserait un ATG putatif 94 codons en aval, entrânant la production d'une protéine délétée de son domaine de liaison à l'actine. Au total, nous avons identifié 17 transcrits alternatifs différents, dont certains jamais décrits dans les banques de données. Le criblage mutationnel de FGD4 dans notre cohorte de patients a permis d'identifier deux nouveaux variants génomiques chez deux patients issus d'unions consanguines : la variation de séquence c.1698G $>\mathrm{A}$ (p.Met566lle) à l'état homozygote dans l'exon 14 chez un patient libanais et la transition c. $2149 \mathrm{G}>\mathrm{A}$ (p.Val717Met) dans l'exon 17 à l'état hétérozygote chez un patient 
algérien. L'absence de la variation de séquence dans des populations témoins, ainsi que la ségrégation des mutations dans les pedigrees sont en faveur de mutations faux-sens. Chez le patient algérien, il est probable qu'une seconde mutation composite reste à identifier dans les régions UTR, promotrices ou introniques. Discussion : FGD4 vient s'ajouter au large panel de gènes mutés dans la famille des CMT. L'identification de deux nouvelles mutations dans $F G D 4$ porte à 7 le nombre de mutations décrites dans ce gène nouvellement rapporté. Ce résultat confirme que les mutations dans FGD4 sont probablement fréquentes et à rechercher en priorité chez des patients atteints de CMT4. Par ailleurs, il semble que les mutations affectent tous les domaines fonctionnels de la protéine, et pas seulement son domaine à activité catalytique RhoGEF (domaine DH). Ceci est à mettre en relation avec les résultats de nos analyses transcriptionnelles qui montrent que de nombreux transcrits alternatifs existent, codant des protéines similaires, mais présentant des combinaisons différentes des divers domaines fonctionnels. Ces résultats laissent penser que cette protéine pourrait avoir des fonctions et des localisations diverses au sein de la cellule.

Mots-clés : Charcot-Marie-Tooth, mutations, transcriptionnel.

- 422 / 462. MYOPATHIE MYOTUBULAIRE DUE À L'INSERTION D'UNE SÉQUENCE ALU DANS L'EXON 13 DU GÈNE MTM1 P. Hilbert (1), J. Jaumotte (1, 2), P. Van den Bergh (3), Ch. Debauche (4), $\mathrm{Ch}$. Verellen-Dumoulin $(1,3)$

(1) Centre de Génétique Humaine, Institut de Pathologie et de Génétique, Gosselies, Belgique; (2) Département de pédiatrie, Cliniques Universitaires Saint-Luc, Bruxelles, Belgique; (3) Centre neuro-musculaire, Cliniques Universitaires Saint-Luc, Bruxelles, Belgique ; (4) Unité de néonatologie, Cliniques Universitaires Saint-Luc, Bruxelles, Belgique

La myopathie myotubulaire liée au chromosome X (XLMTM) est une myopathie congénitale caractérisée par une faiblesse musculaire modérée à sévère. La forme classique (sévère) observée chez les nouveau-nés est caractérisée par une hypotonie importante conduisant à un décès précoce ou nécessitant une ventilation assistée chronique. La maladie est héritée selon le mode récessif lié à l'X. Le gène responsable code pour une protéine de 603 acides aminés, la myotubularine. Plus de 200 mutations différentes (faux-sens, non-sens, délétions, mutations d'épissage...) ont actuellement été décrites. Nous présentons ici le cas d'un nouveau-né atteint d'une forme sévère de la maladie. Les parents d'origine turque sont consanguins. Un léger hydramnios est constaté en fin de grossesse. L'accouchement a lieu par césarienne à 38 semaines. Le score d'APGAR est 0/0/6. L'enfant est intubé et nécessite ensuite une ventilation assistée avec de multiples essais de sevrage. On observe une hypotonie généralisée majeure avec absence de réflexes ostéo-tendineux. La biopsie musculaire est compatible avec le diagnostic de myopathie myotubulaire. L'enfant décède a 6 mois dans un contexte d'infection respiratoire et de malaises cardiaques récidivants. Lors de l'analyse moléculaire du gène, l'amplification de l'exon 13 donne un fragment de taille anormalement élevée par rapport à la taille attendue. La séquence de l'amplicon montre que l'exon 13 est interrompu par une séquence Alu Ya5. La duplication typique au site d'insertion ainsi que la taille de la queue polyA de la séquence Alu permettent de conclure que cet élément provient d'une rétrotransposition. L'étude du cDNA montre que l'insertion provoque un épissage anormal avec perte complète de l'exon 13 . Nous décrivons ici le premier cas de myopathie myotubulaire dû à l'insertion d'une séquence Alu dans la région codante du gène MTMI.

Mots-clés : MTM1, alu, myopathie myotubulaire.

口423 / 467. ANALYSE DES GÈNES PHD, HIF ET FIH DANS UNE SÉRIE DE PATIENTS ATTEINTS DE POLYGLOBULIE * IDIOPATHIQUE *

R. Carcenac (1), B. Gardie (1), C. Ladroue (1), S. Gad (1), O. Hermine (2), J.D. Rain (3), A. Baruchel (4), P. Boivin (5), A. Hummel (6), F. Fakhouri (6), S. Giraud (7), J. Pouyssegur (8), S. Richard $(1,9)$

(1) Génétique Oncologique EPHE, CNRS FRE-2939, Institut de cancérologie Gustave Roussy, Villejuif, et Faculté de Médecine Paris-Sud, Le Kremlin-Bicêtre, France ; (2) Service d'Hématologie Adultes, Hôpital NeckerEnfants Malades, Paris; (3) Laboratoire d'Hématologie, Hôpital Saint-Louis, Paris ; (4) Service de Pédiatrie Hématologique, Hôpital SaintLouis, Paris; (5) Consultation d'Hématologie Clinique, Hôpital Beaujon, Clichy: (6) Service de Néphrologie, Hôpital Necker-Enfants Malades, Paris ; (7) Laboratoire de Génétique, Hôpital Herriot, Lyon ; (8) Laboratoire de Signalisation, Biologie du développement et cancer, CNRS UMR 6543, Centre A.-Lacassagne, Nice; (9) Consultation d'Oncogénétique Spécialisée AP-HP INCa, Service d'Urologie, CHU de Bicêtre, Le Kremlin-Bicêtre et Service de Néphrologie, Hôpital Necker, Paris, France
Introduction : Le nombre de globules rouges dans le sang est étroitement régulé par un mécanisme de détection du taux d'oxygène circulant. Lorsque le rein perçoit une baisse d'oxygène, il induit une production d'érythropoiétine (EPO) qui augmente la production de globules rouges. Des études sur la régulation de l'EPO ont permis d'identifier le facteur de transcription inductible par cette baisse d'oxygène, HIF (Hypoxia Inducible Factor), qui joue un rôle clé dans la réponse cellulaire à l'hypoxie (érythropoïèse, glycolyse, angiogenèse). En condition de normoxie, la sous-unité 1-alpha de HIF est hydroxylée par les Prolyl Hydroxylases (PHD) au niveau des résidus Proline 402 et 564 sur son domaine sensible à l'oxygène (ODD, Oxygen Degradation Domain). Cette hydroxylation permet sa fixation à la protéine VHL (pVHL) qui va entraîner son ubiquitination. HIF1-alpha est ensuite rapidement dégradé dans le protéasome et sa quantité reste indétectable dans le cytoplasme. HIF1-alpha est également hydroxylé au niveau d'une asparagine (Asn 803) par le facteur FIH (Factor Inhibiting HIF) qui inhibe son activité transcriptionnelle. En situation d'hypoxie, PHD et FIH n'hydroxylent plus HIF1-alpha qui n'est plus reconnu par pVHL, n'est donc pas ubiquitiné et se retrouve stabilisé. Il passe dans le noyau où il se conjugue à HIF1-beta pour former un facteur de transcription actif qui va transactiver les éléments de réponse à l'hypoxie (HRE) présents sur les promoteurs des gènes cibles dont l'EPO. Ainsi HIF peut réguler la transcription de l'EPO et donc sa production, en relation étroite avec la concentration en oxygène. Récemment il a été décrit que des membres d'une famille atteints de polyglobulie due à un excès de production d'EPO présentaient une mutation germinale faux-sens du gène PHD2 (p.Pro317Arg). Cette protéine PHD2 mutée a perdu la capacité d'hydroxyler HIF1-alpha. Des mutations particulières du gène $V H L$ ont également été décrites dans ce même type de pathologie. Des anomalies dans la voie de régulation de HIF peuvent donc entraîner une surproduction d'EPO et une polyglobulie. Nous nous sommes intéressés à tous les acteurs de régulation de la voie HIF dans une série de patients atteints de polyglobulie inexpliquée. Matériel et Méthodes: Nous avons analysé une série de 23 patients suivis en Hématologie dont les prélèvements ont été effectués après recueil du consentement. Des mutations ont été recherchées par séquençage direct des gènes PHD1, PHD2, PHD3, FIH dans leur totalité, et HIFI-alpha (exons 9, 12 et 15) ainsi que HIF2-alpha (exons 9, 12 et 16). Par mutagenèse dirigée nous avons ensuite produit des mutants de PHD2 dont nous avons étudié la fonctionnalité in vitro à l'aide d'un gène rapporteur luciférase sous contrôle du promoteur de l'EPO. Résultats-Discussion: Nous avons identifié 5 variants nucléotidiques dans le gène $P H D 2: c .471 \mathrm{G}>\mathrm{C}$ (p.Gln157His), c.599C >A (p.Pro200Gln), c.760G $>$ C (p.Asp254His), c.1112G $>$ A (p.Arg371His) et c.1192C $>$ T (p.Arg398X) que nous nommerons respectivement Q157H, P200Q, R371H, D254H et R398X. Aucune anomalie n'a été mise en évidence dans les autres gènes analysés. Le test luciférase montre que les protéines PHD2 qui portent la substitution D254H ou le codon stop en R398 ont perdu la capacité de réguler HIF et sont donc clairement délétères. Les codons 254 et 398 étant impliqués dans la stabilisation de PHD2 sous forme d'homotétramère, une mutation à leur niveau entraîne de façon compréhensible une perte de fonction de la protéine. À l'inverse, les mutants Q157H, P200Q et R371H ne semblent pas être délétères car ils entraînent une inactivation de HIF comparable à la protéine PHD2 sauvage avec le test luciférase. Les codons 200 et 371 sont très conservés et il ne s'agit pas de polymorphismes, contrairement au variant Q157H que nous avons retrouvé dans une population contrôle avec une fréquence de $3 \%$. Nous allons donc affiner nos tests de fonctionnalité afin de déterminer ce qui différencie ces mutants.

Mots-clés : polyglobulie, PHD2, mutation.

\section{IP424 / 473. NOUVELLES MUTATIONS DU GÈNE LMNA DANS DES CAS SPORADIQUES OU FAMILIAUX DE SYNDROMES PROGÉROIDES}

V. Esteves-Vieira (1), A. Boyer (1), D. Renard (2), D. Bessis (2), M. Chaouch (3), I. Boccaccio (4), P. Roll (5), C. Navarro (4), A. Munnich (6), M. Lemerrer (6), A. Chauvat (7), P. Bourgeois (1), N. Levy (1, 4), A. De Sandre-Giovannoli $(1,4)$

(1) Laboratoire de Génétique Moléculaire, CHU de la Timone, Marseille ; (2) Département de Neurologie, Hôpital Guy-de-Chauliac, CHU Montpellier ; (3) Neurologie, Hôpital Ben-Aknoun, Alger, Algérie ; (4) UMR910, Faculté de Médecine de Marseille: (5) Service de Biologie Cellulaire, CHU La Conception, Marseille ; (6) Département de Génétique, Hôpital Necker, Paris ; (7) Service de Cardiologie, CH Victor-Dupouy, Argenteuil

Introduction : Différents syndromes progéroides segmentaires ont été inscrits dans le cadre nosologique des laminopathies systémiques au cours des dernières années. Dans le contexte d'un PHRC National visant à étendre le nombre de mutations et de syndromes inscrits dans ce cadre, nous avons testé des patients évoquant cliniquement une laminopathie 
systémique de manière plus ou moins marquée. Nous rapportons l'identification de 3 cas sporadiques ou familiaux, porteurs de nouvelles mutations dans le gène $L M N A$. Matériel et Méthodes : Les parties codantes du gène LMNA ont été amplifiées à partir d'ADN leucocytaire et analysées par dHPLC et/ou séquençage direct. Les mutations sont décrites selon les règles proposées par la Human Genome Variation Society. Résultats : Le patient 1 est un homme de 31 ans, sans facteurs de risque cardiovasculaire, se présentant avec une aphasie et une hémiplégie droite. Il présente une lipoatrophie, des hyperkératoses localisées, et un nez fin et long. Une lésion ischémique cérébrale étendue associée à des calcifications thalamiques, a été documentée. Le père du patient, atteint d'artériopathies oblitérantes des membres inférieurs, était décédé à 52 ans suite à infarctus du myocarde. L'analyse du gène LMNA chez le patient, puis chez le père, a mis en évidence la mutation hétérozygote c.898G $>$ A (p.D300N). Les transcrits n'ont pas montré d'anomalies de l'épissage. Le patient 2 est un homme de 35 ans présentant une petite taille, des acroostéolyses, une lipoatrophie, un nez fin, une peau fine, une hypertriglycéridémie, une stéatose hépatique et une athérosclérose disséminée. La mutation hétérozygote c.1968G >A, prédite pour être synonyme (p.Q656Q) a été identifiée. Au contraire, des transcrits délétés identiques à ceux retrouvés chez les patients atteints de Progeria typique (p.G608G) ont été observées. L'analyse en Western Blot a permis de confirmer la présence de faibles quantités de progérine. Le patient 3 est un enfant de 3 ans issu d'une union consanguine, présentant une alopécie, une peau fine, des acroostéolyses, des déformations thoraciques, des ROT abolis, une insuffisance staturale, une lipoatrophie et une intelligence normale. La mutation homozygote c.1583C $>\mathrm{T}$ (p.T528M) a été observée. L'analyse immunocytochimique de ses fibroblastes montrait des noyaux polylobulés avec parfois des anomalies de marquage des Lamines A/C et de l'emerine. L'analyse en RT n'a pas montré d'anomalies d'épissage et le western blot a confirmé la présence de Lamines A/C conservées en taille et quantités. Discussion : Une différente substitution hétérozygote du codon 300 , localisée au niveau d'une partie très conservée du domaine coiled-coil, avait été rapportée au préalable dans une famille atteinte de progeria cardiocutanée. La mutation p.D300N ségrége avec le phénotype familial Werner-like, seulement partiellement évocateur chez le patient 1. Le patient 2 démontre que des mutations autres que la p.G608G peuvent activer de manière substantielle le site cryptique d'épissage impliqué dans la Progeria. Une production de progérine à des faibles niveaux semble conduire à la forme atténuée de Progéria observée chez le patient. La mutation T528M avait été décrite à l'état hétérozygote composite chez des patients présentant une Progeria ou une lipodystrophie. Le résidu T528 se situe au niveau du domaine Ig-like et est très conservé entre les lamines de type A et B. Sa mutation est supposée avoir des conséquences sur la dynamique de polymérisation des Lamines. Nos résultats montrent une fois de plus l'hétérogénéité clinique et allélique des laminopathies systémiques. Nous souhaitons souligner aussi le risque élevé d'affections ischémiques aiguës chez les patients mutés, en l'absence des facteurs de risque et en présence d'un phénotype clinique seulement partiellement évocateur. La recherche de mutations de LMNA est donc souhaitable devant tout soupçon diagnostique pour conseil génétique et prise en charge précoce.

Mots-clés : LMNA, lamine, progeria.

שP425 / 475. DES MUTATIONS DU GÈNE CODANT LA MYOPALLADINE, UNE PROTÉINE DE LA BANDE Z DU SARCOMÊRE, SONT RESPONSABLES DE CARDIOMYOPATHIE DILATÉE

\section{Duboscq-Bidot (1), P. Xu (2), P. Charron $(1,3)$, N. Neyroud (1), G.} Dilanian (1), A. Millaire (4), M. Komajda $(1,5)$. E. Villard $(1,6)$

(1) INSERM/UPMC, UMR S621, Paris ; (2) Centre National de Génoty. page, Evry; (3) Département de Génétique, Hôpital Pitié-Salpêtrière, Paris. (4)Service de Cardiologie, hôpital cardiologiques, Lille. (5)Département de Cardiologie, Hôpital Pitié-Salpêtrière, Paris; (6) Centre d'Investigation Biomédicale Pitié-Salpêtrière, AP-HP, Paris

Introduction : La cardiomyopathie dilatée (CMD) est une maladie cardiaque caractérisée par une dilatation ventriculaire et une altération de la fonction contractile. C'est une cause majeure d'insuffisance cardiaque et de transplantation. La CMD est d'origine génétique dans environ $30 \%$ des cas et très hétérogène génétiquement avec plus de 10 gènes impliqués dans la maladie identifiés à ce jour et aucun gène majeur. Malgré ce nombre, beaucoup de gènes restent encore à identifiés dans cette pathologie. Du fait de la grande morbidité associée à la CMD et de la petite taille des familles atteintes l'analyse de liaison génétique est peu adaptée. L'identification des gènes est donc le plus souvent basée sur une approche gène candidat et un criblage mutationnel. Parmi les gènes connus à ce jour, la majorité code pour des protéines de structure du cardiomyocyte. Matériel cardiomyocytaire nous a permis d'identifier le gène codant la myopalladine (OMIM 608517), une protéine associée à la bande $\mathrm{Z}$ sarcomérique, comme candidat potentiel. Nous avons criblé, par séquençage direct, l'ensemble des exons et jonctions introns-exons du gène chez 114 sujets indépendants d'origine européenne ( 65 cas familiaux et 49 cas sporadiques) présentant une CMD. Les mutations ont été analysées sur les plans génétique (conservation inter-espèce, fréquence allélique, coségrégation, liaison génétique) et fonctionnel (immunohistochimie, expression cellulaire hétérologue). Résultats: Nous avons identifié 4 mutations à l'état hétérozygote dans deux familles (R1088H et I83fsX105) et 2 cas sporadiques (V1195 et P1112L). Pour les 3 mutations faux-sens, l'acide aminé substitué est conservé entre les espèces. Aucune des mutations n'est retrouvée dans une population de 400 contrôles sains. Les sujets atteints sont tous porteurs d'une des mutations et la pénétrance chez les porteurs est incomplète, une caractéristique fréquente dans cette pathologie. Enfin, dans la plus grande famille (mutation $1088 \mathrm{H})$ un lod-score significatif $(\mathrm{Z}=2,3$; theta $=0)$ est obtenu pour le calcul de liaison génétique entre la mutation considérée comme marqueur unique et la maladie. L'immunomarquage spécifique de la myopalladine dans le cœur transplanté d'un patient porteur de la mutation $\mathrm{R} 1088 \mathrm{H}$ indique une perte de localisation de la protéine à la bande Z sarcomérique, suggérant un défaut d'interaction ou d'adressage de la protéine mutante. L'expression hétérologue des mutations P1112L et V1195M dans le modèle des cardiomyocytes de rats nouveaux-nés en culture primaire induit une désorganisation structurale rapide des sarcomères associées à une mort cellulaire importante et précoce comparé aux cellules exprimant la myopalladine humaine sauvage. Enfin, la quantification de la fuite transcriptionnelle lymphoblastique des $\mathrm{ARNm}$ issus des deux allèles codant la mutation I83fs X105 indiquent une absence presque totale du transcrit porteur de la mutation et suggère un mécanisme d'haploinsuffisance associé à cette mutation. Conclusion : Nos résultats expérimentaux, tant génétiques, histologiques, que fonctionnels, indiquent que des mutations du gène codant la myopalladine sont responsables de cardiomyopathie dilatée. Ce nouveau gène est impliqué dans 3 à $4 \%$ des cas dans une population d'origine européenne.

Mots-clés : cardiomyopathie, myopalladine, mutation.

\section{D426 / 478. IMPLICATION DES RÉGIONS 5' ET 3'UTR DE GJA12 CHEZ LES PATIENTS ATTEINTS DE LA MALADIE DE PELI- ZAEUS-MERZBACHER SANS MUTATION PLPI (PMLD)}

P. Combes (1, 2), H. El-Hajj (1, 2), E. Bertini (3), O. Boespflug-Tanguy $(1,2,4), C$. Vaurs-Barrière $(1,2)$

(I) INSERM, U 384, Faculté de Médecine de Clermont-Ferrand, F-63003 Clermont-Ferrand, France : (2) Univ Clermont 1 UFR Médecine, Génétique Médicale, F-63001 Clermont Ferrand, France ; (3) Department of Molecular Medecine, Ospedale Pediatrico Bambino Gesu, Scientific Institute, IRCCS, Rome, Italy; (4) CHU Clermont-Ferrand, Centre de référence des leucodystrophies, Service de Génétique Médicale, F-63003 Clermont Ferrand, France

La maladie de Pelizaeus-Merzbacher (OMIM 312080) est une leucodystrophie due à un défaut primitif de myélinisation du système nerveux central (SNC) de transmission liée à l'X impliquant des mutations du gène PLPI. Elle se caractérise par l'apparition précoce de troubles du développement moteur (nystagmus, hypotonie, ataxie, spasticité) associés à un allongement des conductions nerveuses intracérébrales et des signes d'hypomyelinisation sur l'IRM cérébrale. Cependant $20 \%$ des patients ne présentent aucune anomalie du gène $P L P I$ et sont définis comme atteints de Pelizaeus-Merzbacher Like Disease (PMLD). Une hétérogénéité génétique a été démontrée avec l'identification de mutations du gène Gap jonction alpha 12 (GJA12), codant pour la connexine 47 dans un phénotype PMLD de transmission autosomique récessive (OMIM 608804) (Uhlenberg et al., 2004). Les connexines sont des protéines à 4 domaines transmembranaires exprimées à la surface de deux cellules voisines où elles participent à la formation de jonctions communicantes permettant l'échange d'ions et de petites molécules. Dans le SNC, la connexine 47 est exprimée par les oligodendrocytes et permet l'échange de molécules entre les cellules gliales ainsi que le transport radial au sein de la myéline compacte. Nous avons montré que seulement $7.7 \%$ des patients PMLD sont mutés pour le gène GJA12 (Combes et al., in press). Suite à l'étude de l'unique exon codant de GJA12 (exon 2), 36 patients porteurs de mutations GJA12 sont décrits dans la littérature : 29 patients sont porteurs d'une mutation à l'état homozygote, 2 patients sont porteurs de deux mutations hétérozygotes (hétérozygotes composites) et 5 patients ne sont porteurs que d'une seule mutation à l'état hétérozygote. Dans l'hypothèse d'un mode de transmission autosomique récessif pour l'ensemble des patients mutés pour GJA12, nous avons séquencé les régions 5' et 3' UTR de GJA12 chez les 5 patients porteurs d'une seule mutation hétérozygote dans 
la séquence codante. Cette analyse a permis de mettre en évidence une variation à l'état hétérozygote dans la région 5'UTR chez deux patients d'origine italienne (G588 et mt3550) porteurs sur l'autre allèle d'une mutation conduisant à un décalage du cadre de lecture (respectivement c.392delC et c.292_293insGTA). Cette nouvelle variation identifiée dans le 5'UTR n'a pas été retrouvée dans une population de 112 individus sains italiens non apparentés et l'analyse de la ségrégation des deux mutations identifiées dans la famille de la patiente G588 est en accord avec un mode de transmission autosomique récessif. Ce changement nucléotidique est localisé dans une région hautement conservée correspondant au site distal de fixation du facteur de transcription SOX10 qui a récemment été impliqué dans l'activation de la transcription de GJA/2 chez la souris. Ceci est à rapprocher des mutations décrites au niveau du site distal de fixation de SOX10 situé sur le promoteur du gène GJBI (codant pour la connexine 32) et responsables de la neuropathie périphérique de Charcot-Marie (CMTX1). Des études fonctionnelles de l'effet de cette mutation sur la transcription de GJA12 sont en cours pour conclure définitivement à l'implication de celle-ci dans l'expression du phénotype PMLD afin de déterminer l'intérêt d'analyser de façon systématique chez les patients PMLD, non seulement la région codante mais également la région 5'UTR de GJAI2.

Mots-clés : GJA12, Pelizaeus-Merzbacher, leucodystrophie.

- P427 / 481. DÉLÉTION DU GÈNE SACS IDENTIFIÉE PAR ARRAY-CGH DANS UN CAS DE SPASTIC ATAXIA DE CHARLEVOIX-SAGUENAY (ARSACS)

J. Breckpot (1), Y. Takiyama (2), B. Thienpont (1), E. Ortibus (3), K. Devriendt (1)

(1) Centre for Human Genetics, K.U.Leuven, Belgique; (2) Division of Neurology, Department of Internal Medicine, Jichi Medical University, Tochigi, Japan ; (3) Department of Pediatrics, University Hospital Leuven, Belgique

Nous rapportons le cas d'un patient Belge présentant une ataxie cérébelleuse précoce, une spasticité progressive, une hypotrophie musculaire des membres inférieurs, des difficultés d'apprentissage et une surdité modérée. Un caryotype moléculaire effectué par Comparative Genomic Hybridisation (aCGH), démontre une délétion de $1.49 \mathrm{Mb}$ au chromosome $13 \mathrm{q} 12.12$, comprenant le gène SACS. La microdélétion 13q12.12 était de novo et d'origine maternelle. Le séquençage du gène $S A C S$ a révélé une nouvelle mutation hemizygote c.10517T $>\mathrm{C}$ dans exon 9, résultant dans une substitution du résidu 3506 phenylalanine par serine (p.F3506S). Le père est porteur de cette mutation, qui est absente chez 100 contrôles belges. Ceci présente le premier cas de la maladie ARSACS avec une délétion de novo du gène SACS. À part des implications pour le diagnostic de cette maladie, cette observation montre la contribution potentielle des microdéletions dans l'origine des maladies autosomiques récessives.

Mots-clés : microdélétion, ataxie spastique, micropuces.

DP28 / 482. SCORES CLINIOUES DE DÉPISTAGE DE L'HYPER. CHOLESTÉROLÉMIE FAMILIALE CHEZ L'ADULTE ET L'ENFANT. INTÉRÊT POUR LE DIAGNOSTIC PAR GÉNÉTIQUE MOLÉCULAIRE DANS LA POPULATION FRANÇAISE

P. Benlian (1), C. Bernard (1), C. Morel (1), K. Belabbas (1), W. Auriol (1), A. Fredenrich (2), M. Krempf (3), P. Moulin (4), Y. Reznik (5), B. Vialettes (6), J.-F. Labarthe (7), F. Feillet (8), F. Carrat (9), J.-P. Girardet (10)

(1) Laboratoire de Référence pour le Diagnostic Génétique des Maladies Rares, APHP, INSERM UMRS 538, UPMC Paris ; (2) Diabétologie-Endocrinologie, $\mathrm{CHU}$ de Nice ; (3) Endocrinologie, CHU de Nantes; (4) Endocrinologie, Hôpital Louis Pradel, Lyon; (5) Endocrinologie, CHU de Caen; (6) Nutrition, Maladies Métaboliques et Endocrinologie, CHU Timone, Marseille : (7) Pédiatrie, CHRU de Tours ; (8) Médecine Infantile, CHU de Nancy ; (9) UMR-S 707 ; INSERM, Université Paris 6; (10) Gastroentérologie et Nutrition Pédiatrique, Hôpital Armand-Trousseau, APHP, Paris

Introduction : L'hypercholestérolémie familiale (HCF) justifie d'un dépistage chez le sujet jeune avant la survenue des premières manifestations cardiovasculaires, souvent mortelles d'emblée. Bien que le risque cardiovasculaire de l'HF hétérozygote soit multiplié par plus de 50, il reste difficile de distinguer en pratique les formes sévères d'HCF de formes d'hypercholestérolémie plus banales ou multifactorielles. De plus, si le diagnostic par génétique moléculaire offre des arguments de certitude par analyse des trois gènes connus ( $L D L R, A P O B, P C S K 9)$, son rendement en pourcentage de porteurs identifiés « mutés » est réduit (de $80 \%$ à $25 \%$ ) par un dépistage clinique peu discriminant. Des scores diagnostiques basés sur des critères clinico-biologiques ont été développés de manière intuitive ou statistique chez l'adulte (Simon Broome, UK ; Dutch Lipid Clinics, NL; Med Ped, USA), sans équivalent pour l'enfant qui est asymptomatique. Objectifs. Valider par la génétique moléculaire chez 300 adultes et élaborer chez 150 enfants hypercholestérolémiques issus de différentes régions françaises, des scores diagnostiques discriminants basés sur un ensemble de critères clinico-biologiques pondérés. Sujets et Méthodes: Les adultes (LDLC $>1,60 \mathrm{~g} / \mathrm{L}$ ) et les enfants (LDLC $>1,30 \mathrm{~g} / \mathrm{L}$ ) hypercholestérolémiques, et/ou présentant des dépôts vasculaires ou extravasculaires de cholestérol et/ou une histoire familiale évocatrice d'HCF ont été génotypés aux loci $L D L R, A P O B$ et $P C S K 9$ par séquençage (Big Dye Terminator, Applera, France) sur produits de PCR (en aval ou non de la DGGE, BioRad). Les gros réarrangements géniques du LDLR ont été recherchés par Southern Blot ou MLPA (Multiple Ligation-dependent Probe Amplification, MRC-Holland). Les séquences ont été analysées en double à l'aide du logiciel SeqScape v2.5 (Applera, France) pour la détection de variants et du logiciel GenSearch v3.34 (Phenosystems, Belgium) pour l'aide à l'interprétation fonctionnelle; les logiciels Gene Mapper v4.0 (Applera, France) et Coffyalyser v2 (MRC-Holland) ont été utilisés pour le dosage allélique. La validation des scores cliniques a été effectuée par analyse de régression logistique multivariée (SAS Institute, USA). Résultats : Le score Dutch Lipid Clinics basé sur 10 critères clinico-biologiques pondérés a été appliqué chez 220 adultes porteurs et 80 adultes non porteurs de mutations causales d'HCF. Les sujets porteurs de mutations causales de formes dominantes d'HCF ont été détectés avec une sensibilité de $84 \%$ au seuil de « Score $>8$ " (aire sous la courbe ROC $=0,82$ ). Le score moyen des adultes non mutés (Score $=8$ ) était très inférieur au score des adultes mutés (Score $=13, \mathrm{p}<0,0001$ ), offrant une précision diagnostique comparable à celle observée dans la population Hollandaise. Un score spécifique a été élaboré chez 50 enfants porteurs et 50 enfants non porteurs de mutations causales sur la base de trois critères indépendants identifiés parmi 80 testés: LDLC avant et après régime, et notion de traitement parental par statine. Ce score a ensuite été appliqué sur un groupe de 50 autres enfants dont le génotype n'était pas connu. La sensibilité de détection des porteurs était de $90 \%$ avec une aire sous la courbe $R O C=0,92$. Les sujets identifiés comme mutés étaient porteurs de mutations ponctuelles du $L D L R$ dans $90 \%$ des cas, de gros réarrangements géniques du $L D L R$ dans $5 \%$ des cas, de mutations de l'APOB dans $4 \%$ des cas et de mutations de PCSK9 dans $1 \%$ des cas. Conclusion : Sachant qu'en pratique l'HCF relève de traitements préventifs efficaces si débutés précocement, ces scores cliniques testés et validés par la génétique moléculaire, offrent aux praticiens un outil décisionnel précis tant chez l'adulte que chez l'enfant encore indemne de toute pathologie cardiovasculaire. De plus, l'efficience du diagnostic moléculaire est augmentée par un dépistage clinique mieux ciblé sur les individus porteurs d'HCF monogéniques à très haut risque.

Mots-clés : hypercholestérolémie familiale, dépistage, MLPA.

\section{EP429 / 483. PRÉVALENCE DE LA MUTATION MITOCHON-} DRIALE A1555G EN FRANCE

D. Feldmann (1), L. Jonard (1), A.F. Roux (2), C. Lemarechal (3), K. Olszewski (1), C. Francannet (4), R. Couderc (1), F. Denoyelle (5), S. Marlin (6)

(I) Laboratoire de Biochimie, INSERM U587, Hôpital Armand-Trousseau, AP-HP, Paris ; (2) Laboratoire de Génétique Moléculaire-IURC, CHU de Montpellier ; (3) Laboratoire de Génétique Moléculaire, Unité INSERM U613, CHU de Brest; (4) Génétique Médicale, Hôtel-Dieu, ClermontFerrand; (5) Service d'ORL et de Chirurgie Cervico-Faciale, Inserm U587, Hôpital Armand-Trousseau, AP-HP, Paris ; (6) Unité de Génétique Médicale, Inserm U587, Centre de Référence des Surdités Génétiques, Hôpital Armand-Trousseau, AP-HP, Paris, France

La surdité est l'handicap neurosensoriel le plus fréquent de notre population. En effet 1 enfant sur 1000 nait avec un déficit auditif. La surdité est largement hétérogène génétiquement. Des mutations de l'ADN mitochondrial sont responsables de surdités isolées ou syndromiques. Parmi ces mutations, la mutation A1555G dans l'ARN12S se caractérise par son aptitude à potentialiser l'effet ototoxique des aminosides, médicaments largement utilisés. Depuis la mise en évidence de la mutation, celle-ci a été identifiée dans de nombreuses familles de patients atteints de surdité non syndromique. Le phénotype associé à A1555G est extrêmement variable même parmi les membres d'une même famille et la surdité peut s'observer en l'absence de traitement par les aminosides. Enfin les études familiales ont montré la présence de porteurs sains. Cependant l'administration d'aminosides chez des porteurs de la mutation A1555G est toujours suivie de surdité. La pénétrance incomplète ainsi que l'expressivité variable du déficit auditif associées à $\mathrm{A} 1555 \mathrm{G}$ suggère qu'en plus des facteurs 
environnementaux comme la prise d'aminosides, des gènes modificateurs nucléaires ou mitochondriaux modulent le phénotype. Cette mutation a été décrite dans de nombreuses populations et des études récentes en Espagne ont montré que cette mutation était présente chez $15 \%$ des patients atteints de surdité non syndromique et chez $61 \%$ des familles où la surdité était transmise par un ascendant maternel. Une fréquence élevée a aussi été observée en Chine (5.4\% des patients atteints de surdité NS). En conséquence une recherche systématique de cette mutation peut être justifiée dans ces populations. La prévalence de A1555G en France n'étant pas connue, nous avons voulu l'établir dans une large cohorte de patients sourds. Parmi une cohorte de 1880 patients atteints de surdité, 156 patients non apparentés avec une surdité ayant un mode de transmission maternelle et 124 cas sporadiques ayant reçu des aminosides, ont été étudiées. La surdité a été évaluée et d'éventuelles atteintes associées ont été éliminées. La mutation A1555G a été analysée par électrophorèse en gradient dénaturant puis séquençage. La mutation A1555G a été observée à l'état homoplasmique dans seulement 7 familles parmi les 156 avec transmission maternelle soit $4,5 \%$ ce qui est statistiquement différent de la population espagnole ( $7 / 156$ versus $65 / 107)$. Aucun des cas sporadiques ne portait la mutation (0/124 versus 5/98). L'origine des patients porteurs de A1555G était variée et jamais française : Chine, Martinique, Arabe/Israël, Portugal, Iran, Madagascar, Espagne. La surdité était de profonde à légère, le début d'apparition de congénital à 30 ans. Le pourcentage de patients atteints parmi les apparentés maternels était de $50 \%$ (51/102). La faible fréquence de la mutation A1555G observée en France ne justifie pas un screening systématique de cette mutation. La recherche de la mutation devra être réservée aux patients avec un ou plusieurs apparentés maternels atteints. aux patients originaires de pays où la mutation a une forte prévalence et aux enfants de recevoir fréquemment des aminosides.

Mots-clés : A1555G, mutation mitochondriale, surdité.

\section{EP430 / 490. ÉVALUATION DES SITES WEB DE PRÉDICTION IN SILICO DES MUTATIONS D'ÉPISSAGE COMME OUTIL D'AIDE DÉCISIONNELLE EN DIAGNOSTIC GÉNÉTIQUE}

D. Michaux*, C. Dehainault*, C. Mattler, V. Caux-Moncoutier, S. PagèsBerhouet, D. Stoppa-Lyonnet, M. Gauthier-Villars, C. Houdayer, *D. Michaux et $\mathrm{C}$. Dehainault ont contribué de façon égale à ce travail Génétique oncologique, Direction des Systèmes d'Information et de l'Informatique Institut Curie, Paris, France

L'épissage est le processus complexe par lequel les cellules eucaryotes produisent un ARN messager (ARNm) mature à partir d'un pré-ARNm. Il nécessite la reconnaissance des exons, l'excision des introns puis la ligation des exons pour former un transcrit mature. Cette reconnaissance est assurée par des séquences génomiques consensus en cis, dont les plus connues sont les sites donneurs, accepteurs d'épissage et le site de branchement. D'autres séquences consensus ont été décrites, principalement les Exonic Splicing Enhancers (ESE) et les Exonic Splicing Silencers (ESS). Ce sont des séquences exoniques qui favoriseraient (ESE) ou réprimeraient (ESS) l'épissage de l'exon qui les contient et ce par interaction avec des facteurs de transcription spécifiques. Il est maintenant établi que toute modification nucléotidique génomique peut être délétère en affectant l'épissage du pré-ARNm par la perte d'une séquence consensus sauvage ou la création d'une séquence consensus cryptique. Cependant, pour des raisons de temps et de coût, il n'est pas envisageable d'effectuer une étude de transcrits pour chaque variant nucléotidique. Par conséquent, un compromis doit être trouvé entre le temps et le coût d'une telle étude et le risque de manquer une mutation délétère. Nous avons donc évalué les performances de certains outils disponibles en ligne qui permettent de prédire in silico l'impact sur l'épissage des modifications nucléotidiques. Trente six patients non apparentés atteints de rétinoblastome, sans mutation délétère identifiée mais porteurs de variants de signification inconnue du gène $R B 1$ ( 28 introniques et 8 exoniques) ont été inclus. Pour ces 36 patients, une étude de transcrits systématique a été réalisée, à partir de lignées cellulaires traitées en puromycine, afin de visualiser les transcrits résultant de mutations tronquantes et instables du fait du phénomène NMD. L'impact sur l'épissage de ces 36 variants de signification inconnue a ensuite été modélisé à l'aide de 5 sites (Splice Site Prediction by Neural Network, Splice Site Finder, MaxEntScan, ESE Finder and RESCUE-ESE) et les résultats des analyses in silico ont été comparés à ceux des études de transcrits. De façon attendue, 18 modifications nucléotidiques modifiant les sites canoniques d'épissage AG/GT ont correctement été caractérisées in silico comme délétères. Sur 18 modifications nucléotidiques introniques situées à $+/-60$ nucléotides d'un site d'épissage physiologique, 16 mènent, in vitro, à un défaut d'épissage et 13 d'entre elles ont été correctement classées, in silico, comme délétère par au moins un des sites testés. En été détecté par aucun des 5 sites et les 2 restantes ont été correctement caractérisées en tant que neutre. Nos résultats sur RB1 montrent que $90 \%$ des UV altérant l'épissage peuvent être détectés par l'utilisation d'une combinaison d'outils de prédiction complémentaires. En effet, la faiblesse d'un outil in silico peut être compensée par la force d'un autre. Bien que les prédictions in silico ne soient pas suffisantes pour classer les UV comme délétères ou neutres, elles peuvent être utilisées en routine diagnostique comme outil d'aide décisionnelle pour guider le généticien vers les études de transcrit pertinentes.

Mots-clés : mutations d'épissage, modélisation in silico, outils en ligne.

- 431 / 492. LA TECHNIQUE MLPA (MULTIPLEX LIGATIONDEPENDENT PROBE AMPLIFICATION) : UNE ALTERNATIVE À LA MÉTHODE DE SOUTHERN POUR LA DÉTECTION DES LARGES RÉARRANGEMENTS DU GÈNE CYP21 IMPLIQUÉS DANS LE DÉFICIT EN 21-HYDROXYLASE

V. Tardy (1, 2), M. Manigand (1), L. Michel-Calemard (1), N. BoutryKryza (1), Y. Morel $(1,2)$

(1) Hospices Civils de Lyon, Endocrinologie Moléculaire et Maladies Rares, Centre de Biologie et de Pathologie Est, Bron, F69677, France; (2) Université Lyon 1, Faculté de médecine Laënnec, Lyon, F69003, France

Le déficit en 21-hydroxylase est dû à des mutations du gène CYP21 situé dans une région $6 \mathrm{p} 21.3$ entre les loci HLA B et HLADR. Le gène CYP21 se trouve dans un fragment de $35 \mathrm{~kb}(C 4 B-C Y P 21-T N X B)$ dupliqué en tandem (C4A-CYP2IP-TNXA) avec son pseudogène CYP2IP homologue à $98 \%$. Cette grande homologie explique la fréquence de réarrangements importants (duplication, délétion, conversion génique) ou d'échanges constants entre CYP21P et CYP21 rendant CYP21 non fonctionnel. Les réarrangements très étudiés dans les années 90 (Southern, électrophorèse en champ pulsé) restent complexes : dus le plus souvent à des crossingover entre les exons 4 et 8 des gènes CYP21 avec perte ou gain d'un fragment de $35 \mathrm{~kb}$, ils donnent un gène hybride CYP2IP/CYP21 non fonctionnel. Le but de ce travail est de remplacer la méthode de référence, le Southern, par la technique MLPA pour rechercher ces larges lésions responsables du déficit en 21-hydroxylase dans $20 \%$ des cas. Méthodes : Le kit Salsa P050 (MRC Holland) est utilisé. Il renferme 14 sondes spécifiques d'exons présents dans les gènes d'intérêt $(5$ pour $C Y P 21,3$ pour $C Y P 21 P$, 1 pour $C 4 A, 1$ pour $C 4 B, 3$ pour $X B$ et 1 pour $C R E B L$ ) et 20 sondes spécifiques de loci contrôles. Etapes de la MLPA : hybridation spécifique des sondes marquées par un fluorochrome, ligation, amplification des produits par PCR grâce à un couple d'amorces universelles, séparation par électrophorèse sur un séquenceur capillaire ABI. Pour chaque sujet testé, la hauteur du pic de chaque exon d'intérêt est comparée à celle de chaque locus contrôle et ce rapport R1 est lui-même comparé à celui $\mathrm{R} 2$ obtenu chez le sujet normal. Si R1/R2 $<0.7$ exon délété, si $0.7<\mathrm{R} 1 / \mathrm{R} 2<1.3$ profil normal, si R1/R2>1.3 exon dupliqué. Depuis 2006, nous avons testé 80 sujets (110 chromosomes) déjà analysés par Southern ainsi que 430 nouveaux sujets (619 chromosomes). La recherche des mutations de CYP21 situées sous les 5 sondes est toujours réalisée (séquençage ou RLFP). Résultats : les mêmes résultats qu'avec le Southern sont obtenus pour les 110 chromosomes témoins sur le nombre de copies de CYP2l et de CYP21P; les délétions de CYP21 sont différenciées des conversions géniques (nombre de copies de CYP21P identique si délétion, 1 copie supplémentaire de $C Y P 2 I P$ si conversion) et le gène résultant hybride CYP21P/CYP21 est bien détecté. Les bornes d'une duplication sont parfois difficiles à définir (problème du rapport R1/R2 à 1.3). En revanche, le nombre de copies de $C 4 A$ ou $C 4 B$ sur 37 chromosomes discorde avec le Southern (sonde unique, crossing-over entre C4A et C4B). Parmi les 619 chromosomes testés : la MLPA a déterminé la nature de la large lésion de CYP21 (délétion, conversion) suspectée sur 92 chromosomes; elle l'a diagnostiquée (7) ou exclu (29) pour 36 chromosomes où le séquençage ne permettait pas de trancher. Sur 29 chromosomes indemnes de toute lésion d'après le phénotype mais porteurs d'une mutation sévère avec un aspect dupliqué au séquençage (allèle normal supérieur à allèle muté), la MLPA a détecté 2 copies de CYP2I confirmant ainsi la non pathogénicité de l'allèle. Chez 3 sujets non apparentés, la MLPA a diagnostiqué une délétion de CYP21 sur un allèle et une duplication de CYP21 sur l'autre allèle, expliquant la non détection de la délétion par séquençage. Enfin, la MLPA a confirmé l'absence de lésions initialement conclue au séquençage (possibles hétérozygotes d'après le test ACTH, conjoints). Conclusion : la technique MLPA est un outil fiable pour diagnostiquer les larges lésions de CYP21, notamment à l'état hétérozygote, à condition d'avoir recherché les mutations de CYP21 sous les sondes par séquençage. Elle peut être proposée en plus du séquençage pour le conseil génétique et apporter une explication aux discordances génotype-phénotype. Ses limites actuelles 
sont dues à la complexité de la région (difficulté à borner une duplication, résultats peu fiables du $\mathrm{C} 4$ ) et nécessitent de reconsidérer le choix des sondes des gènes $C 4$.

Mots-clés : déficit en 21-hydroxylase, larges réarrangements, technique MLPA (Multiplex Ligation-dependent Probe Amplification).

\section{DP32 / 494. LE SYNDROME ATRX : ASPECTS CLINIQUES ET MOLÉCULAIRES}

C. Badens (1), C. Lacoste (1), N. Martini (1), J. Hornung (1), P. Edery (2), B. Thienpont (3), K. Devriendt (3), N. Philip (4), N. Levy (1) et les cliniciens du réseau DHOS sur le retard mental

(1) Laboratoire de Génétique Moléculaire, CHU de la Timone, Marseille ; (2) Service de Cytogénétique, CHU E.-Herriot, Lyon; (3) Center for Human Genetics, University Hospital Leuven, Leuven, Belgique ; (4) Service de Génétique Clinique, CHU de la Timone, Marseille, France

Le syndrome ATRX associe dans sa forme typique, un retard mental sévère, une alpha-thalassémie mineure, des anomalies génitales et une dysmorphie faciale caractéristique. Des descriptions récentes ont permis d'élargir ce phénotype à des formes avec retard mental modéré ou léger, des formes sans alphathalassémie ou sans anomalies génitales. Au final, seule la dysmorphie faciale est constante et spécifique, même si elle peut être discrète ; elle a pour caractéristique un epicanthus, un nez petit, des narines antéversées, une bouche triangulaire. Des signes secondaires sont retrouvés, certains fréquemment comme une épilepsie, d'autres beaucoup plus rarement comme des anomalies cardiaques ou rénales. Des mutations du gène $A T R X$ sont responsables de ce syndrome. Il code pour une protéine dont la fonction n'est pas précisément connue mais qui intervient dans le remodelage chromatinien et comporte au moins 2 domaines fonctionnels : un domaine en doigt de $\mathrm{Zn}$ et un domaine à activité hélicase. Une étude récente montre que l'expression d'ATRX est déterminante pendant le développement puisque, chez les femmes porteuses de mutation à l'état hétérozygote, il s'opère une sélection des cellules exprimant correctement ATRX pendant cette période. Cette sélection aboutit à un biais d'inactivation du chromosome X en faveur de l'X portant l'allèle intact, ce qui explique que les femmes conductrices sont indemnes de l'affection. Le domaine en doigt de $\mathrm{Zn}$ est le premier hot spot de mutations décrit dans ATRX et regroupe $43 \%$ des mutations dans moins de $5 \%$ de la séquence codante. La grande majorité des mutations caractérisées sont des mutations faux sens mais on retrouve aussi des délétions/insertions ou des mutations de sites d'épissage. Une cohorte de 26 patients diagnostiqués dans notre laboratoire, nous a permis de mettre en évidence un second hot spot dans la région à activité hélicase, qui contient $33 \%$ des mutations dans $10 \%$ de la séquence codante. Une étude collaborative nous a également permis de décrire deux patients présentant des duplications intragéniques concernant plusieurs exons, élargissant ainsi le spectre de mutation rencontrée dans le syndrome ATRX. Le diagnostic moléculaire de ce syndrome est donc complexe puisqu'il s'agit d'un gène de grande taille ( 35 exons dont un de $3 \mathrm{~kb}$ ) avec un spectre de mutations très large, incluant des mutations ponctuelles mais aussi des délétions et des duplications. En 2007, nous avons initié la création d'une base de données spécifiques des mutations du gène ATRX, répertoriant toutes les mutations parues dans la littérature (environ 80 mutations). Dans le syndrome ATRX, aucune corrélation génotype-phénotype n'a pu être clairement mise en évidence en dehors des anomalies uro-génitales graves dans les mutations tronquantes de la partie $3^{\prime}$ du gène. Toutefois, à partir des données cliniques des patients caractérisés dans le laboratoire, nous avons pu montrer qu'une sévérité moindre était rattachée aux mutations siégeant dans la région hélicase, en particulier pour le développement moteur et pour les anomalies génitales. Ceci peut s'expliquer par l'existence de 2 transcrits physiologiques: un transcrit correspondant à l'intégralité du gène et un transcrit tronqué ne comportant que le domaine en doigt de $\mathrm{Zn}$. Le second transcrit serait conservé dans les cas de mutations du domaine hélicase ce qui produirait un phénotype atténué. Bien que le syndrome ATRX soit essentiellement rapporté chez des garçons, nous avons décrit un authentique syndrome ATRX chez une fillette porteuse de la mutation récurrente R246C et d'un biais d'inactivation, total mais inversé par rapport aux femmes porteuses indemnes c'est-à-dire en faveur du chromosome X muté. Même si le tableau clinique s'est élargi à des formes atténuées, le syndrome ATRX reste, avec un taux de détection de l'ordre de $20 \%$, un retard syndromique lié à l'X relativement repérable, en particulier du fait de la dysmorphie faciale caractéristique.

Mots-clés : syndrome ATRX, retard mental lié à l'X, biais d'inactivation du chromosome $\mathrm{X}$

口433 / 498. ANALYSE DU GÈNE GDAPI DANS LES NEUROPATHIES PÉRIPHÉRIQUES HÉRÉDITAIRES SENSITIVES ET MOTRICES : CONSEQUENCES EN TERME DE STRATÉGIE D'EXPLORATION MOLÉCULAIRE ET DE CONSEIL GÉNÉTIQUE N. Palot Bonello (1), N. Martini (1), J. Pouget (2), S. Attarian (2), A. Mégarbané (3), N. Lévy $(1,4), \mathrm{R}$. Bernard $(1,4)$
(1) Département de Génétique médicale Laboratoire de Biologie Moléculaire CHU Timone Marseille; (2) Service des maladies neuromusculaires CHU Timone Marseille; (3) Unité de Génétique Médicale, Faculté de Médecine, Université Saint-Joseph de Beyrouth, Paris, France ; (4) Inserm UMR 910 Faculté de médecine Marseille (4)

Introduction: Les neuropathies périphériques héréditaires sensitives et motrices ou maladie de Charcot-Marie-Tooth (CMT) constituent un groupe de pathologies génétiquement très hétérogène. Différents sous-types de CMT sont identifiés en fonction des conclusions de l'examen électrophysiologique et du mode de transmission de la pathologie. Pour chacun de ces sous-types, une stratégie spécifique d'exploration moléculaire a été établie. Le « réseau des Laboratoires de Génétique Moléculaire pour le diagnostic des maladies neuro-musculaires, neuro-dégénératives, neurosensorielles et des retards mentaux » (DHOS réseau ministériel 2003) a évalué entre autres la place du gène GDAPI dans les stratégies d'exploration moléculaire. L'analyse du gène GDAPI est donc à ce jour incluse dans le schéma d'exploration des formes récessives de CMT axonale et démyélinisante et se discute de ce fait chez les cas sporadiques. Matériels et Méthodes: Nous avons réalisé une étude rétrospective sur une cohorte de 135 individus non apparentés tous suspects de CMT chez lesquels l'analyse du gène GDAPI a été effectuée par séquençage direct. Résultats : Nous avons mis en évidence des variations de séquence dans le gène GDAPI chez 9 patients avec la présence de deux variations de séquence ou d'une mutation à l'état homozygote chez seulement 3 d'entre eux. En effet, 6 d'entre eux ne présentent qu'une variation de séquence à l'état hétérozygote ; 5 sont de type faux-sens et 1 non-sens. 5 de ces variations de séquence ne sont rapportées, à ce jour, ni dans la littérature ni dans les bases de données. Discussion : Nous discutons d'une part la pathogénicité des variations de séquence non décrites mises en évidence, sur des arguments en partie issus des analyses in silico. D'autre part, nous avons mis en évidence la mutation c.358C >T (p.Arg120Trp) à l'état hétérozygote chez 3 patients présentant un phénotype sévère. Cette mutation a été rapportée par plusieurs équipes comme responsable de CMT2 en association à d'autres mutations. Par ailleurs, dans la même étude les auteurs rapportent que deux mutations dont cette même variation de séquence (p.Arg120Trp et p.Trp157Pro) seraient potentiellement responsables de formes dominantes de CMT2. Sur la base conjointe de ces données de la littérature et de nos propres résultats, il semblerait donc que des mutations dans le gène GDAPl puissent être responsables de formes dominantes de CMT, avec chez les 3 patients de notre étude un phénotype sévère. Toutefois, la recherche complémentaire dans les autres gènes de CMT reste à discuter dans ces familles, et l'hypothèse d'un mécanisme éventuel de digénisme ne peut être totalement exclue. Conclusion: Cette étude nous a permis de discuter le caractère pathogène des variations de séquence de GDAP1 non rapportées dans la littérature et les bases de données. À l'heure actuelle, les incertitudes d'interprétation pour certaines mutations de ce gène rendent le conseil génétique extrêmement délicat. De plus, la place de l'analyse du gène GDAPl semble devoir être rediscutée et avoir sa place dans les stratégies d'explorations moléculaires de toutes les formes de CMT : formes axonales comme démyélinisantes, et ceci qu'elles soient transmises sur un mode récessif ou dominant.

Mots-clés : Charcot-Marie-Tooth, GDAPI, stratégie d'exploration moléculaire.

P434 / 499. LA FRÉQUENCE DE MUTATIONS C282Y ET H63D DU GENNE HFE DANS LA POPULATION ROUMAINE

P.M. Voicu (1), C. Cojocaru (2), E. Petrescu (1), M. Covic (3), M. Rusu (1)

(1) Département de Biochimie, Université de Médecine et Pharmacie Gr.T. Popa Iasi, Roumanie: (2) Service de Gastro-Entérologie, CHU St.-Spiridon Iasi, Roumanie ; (3) Département de Génétique Humaine, Université de Médecine et Pharmacie Gr.T. Popa Iasi, Roumanie

Objectif : L'hémochromatose HFE ou de type I est la forme la plus fréquente d'hémochromatose héréditaire dans la population caucasienne. Elle se transmet de manière autosomique récessive et atteint entre 1 individu sur 200, et jusqu'à 1 individu sur 400 dans les populations d'origine nordouest européenne. La maladie, caractérisée par une surcharge en fer importante, est associé à deux mutations dans le gène $H F E$ : la mutation $\mathrm{C} 282 \mathrm{Y}$ (substitution d'une cystéine par une tyrosine) situé dans l'exon 4, et la mutation H63D (substitution d'une histidine par une arginine) dans l'exon 2. De nombreuses études ont montré que $60-100 \%$ des malades d'hémochromatose sont homozygotes pour la mutation $\mathrm{C} 282 \mathrm{Y}$ et environ $4 \%$ sont hétérozygotes composites $\mathrm{C} 282 \mathrm{Y} / \mathrm{H} 63 \mathrm{D}$. La fréquence allélique de la C282Y dans les populations européennes diminue selon un gradient nordouest sud-est. Les fréquences le plus importantes ont été retrouvées en Irlande $(14 \%)$ et en Bretagne (7-8\%). Les plus faibles ont été retrouvées 
dans le sud de l'Italie (1\%). L'objectif de cette étude a été de déterminer la fréquence de $\mathrm{C} 282 \mathrm{Y}$ et $\mathrm{H} 63 \mathrm{D}$ dans la population Roumaine, non étudiée à ce jour. Matériel et Méthode : Nous avons cherché la mutation C282Y chez 149 individus et la mutation H63D chez 99 individus originaires des départements du nord-est de la Roumanie par amplification-restriction. L'ADN a été amplifié à partir de sang déposé sur papier buvard en utilisant deux couples d'amorces pour chaque mutation, dans un thérmocycleur Eppendorf. Les séquences des amorces utilisées pour la mutation C282Y ont été : le premier couple 5'-TGGCAAGGGTAAACAGATCC-3'(sens) et 5'- CTCAGGCACTCCTCTCAACC-3' (antisens) et le deuxième couple 5'- ACCTCTTCAGTGACCACTCTACGGTGTCGG-3' (sens) et 5'-TGACTAGGGTGCCAGACGGTGAGGGCTCTA-3' (antisens). Les amorces pour la H63D ont été : le premier couple 5'-ACATGGTTAAGG CCTGTTGC-3' (sens) et 5'-GCCACATCTGGCTTGAAATT-3' (antisens) et le deuxième couple 5'-ACATGGTTAAGGCCTGTTGC-3' (sens) et 5'-GCCACATCTGGCTTGAAATT-3' (antisens). La digestion enzymatique a été réalisée avec les enzymes RsaI pour la C282Y et MboI pour la H63D, la visualisation des fragmentes obtenus étant faite sur gel d'agarose $3 \%$. Résultats : La distribution de génotypes C282Y a été CC- $95,3 \%$, CY-4,02 \% et YY-0,67 \% et les fréquences allèliques de l'allèle C- $97,31 \%$ et Y-2,68\%. Concernant la H63D : la fréquence des génotypes a été HH$76,76 \%$, HD- $20,2 \%$ et DD-3,03 \% et les fréquences allèliques de l'allèle $\mathrm{H}-86,86 \%$ et D-13,13\%. Discussion et Conclusion : La fréquence allélique de $2,68 \%$ de la $\mathrm{C} 282 \mathrm{Y}$ présente en Roumanie s'inscrit dans les valeurs faibles trouvées pour cette mutation dans l'Europe Centrale et de l'Est ( $4 \%$ en Slovenie $3,8 \%$ en Autriche, 3,4 en Hongrie, 3,3 en Croatie, $1,6 \%$ en Serbie et Montenegro, $0 \%$ en en Bulgarie et $0,3 \%$ en Grèce). La valeur de $13,13 \%$ pour la H63D en Roumanie est proche des fréquence de cette mutation dans l'Europe de l'Est : $11,1 \%$ en Autriche, $14,4 \%$ en Hongrie, $14,5 \%$ en Slovenie et Croatie, $14,6 \%$ en Grèce. Nous avons remarqué une différence importante entre la Roumanie et la Bulgarie pour la H63D, de $13,13 \%$ contre $23 \%$, (la Bulgarie étant située à la frontière du sud de la Roumanie). Nous envisageons une amélioration de résultats de cette étude par l'augmentation de nombre des individus analysés et par l'extension de l'étude aux autres régions du pays.

Mots-clés : hémochromatose HFE1, fréquence C282Y, fréquence H63D.

E435 / 500. EXPLORATION MOLÉCULAIRE DE MALADIES ORPHELINES D'ORIGINE GÉNÉTIQUE: CONSÉQUENCES POUR LE DIAGNOSTIC ET LE CONSEIL GÉNÉTIQUE

A. Amouri (1), S. Abdelhak (1), Pour l'Unité de Recherche Exploration Moléculaire de maladies Orphelines d'Origine Génétique (1) et collaborateurs (2)

Unité de recherche EMGOOD : Etude des Maladies Orphelines d'Origine Génétique, Institut Pasteur de Tunis, Tunisie

La population tunisienne est caractérisée par son hétérogénéité génétique et un fort taux de consanguinité. Il est bien établi qu'il peut y avoir une relation de cause à effet entre la consanguinité et la prévalence des affections autosomiques récessives. Avec les progrès de la recherche en biologie moléculaire, de plus en plus de défauts mono ou polygéniques ont été identifiés. Nous rapportons ici, les résultats des explorations moléculaires réalisées dans le cadre d'études de maladies rares telles que l'anémie de Fanconi, l'épidermolyse bulleuse, l'hyperkératose palmoplantaire. Malgré l'hétérogénéité de ces affections, nous avons élaboré une stratégie unique pour leur investigation génétique et moléculaire. Dans un premier temps, une collecte des données cliniques et généalogiques est faite en collaboration avec des cliniciens et des biologistes des différentes spécialités parallèlement à une fouille de données dans les bases de données en biologie. Après le consentement éclairé, et collecte du matériel biologique, le génotype des patients et de leurs familles est déterminé pour des marqueurs polymorphes de type microsatellite encadrant le ou les gènes déjà connus comme étant impliqués dans la maladie qui les affecte. La liaison au gène d'intérêt est déterminée par homozygotie par descendance. La confirmation de la liaison est effectuée par une recherche de mutation dans la région codante du(es) gène(s) considéré(s). Malgré la taille relativement réduite de la population tunisienne, une hétérogénéité clinique et génétique est souvent observée. En tenant compte du contexte, historique, socio-culturel et économique du sud du bassin méditerranéen, nous proposons une stratégie d'investigation des maladies génétiques et des outils moléculaires simples et peu coûteux pour le diagnostic dans les populations consanguines.

Contribution égale selon un ordre alphabétique : Sonia Abdelhak, Abdelmajid Abid, Imène Arfa, Manel Bali, Emna Barkaoui, Mbarka Bchetnia, Héla Ben Abid, Slim Ben Ammar, Nizar Ben Halim, Mariem Ben Rekaya,
Chérif, Ibtissem Chouchane, Imen Dorboz, Anis Ghazel, Haifa Jmel, Selma Kassar, Rym Kéfi, Chokri Maktouf, Olfa Messaoud, Habib Messai, Sonia Nouira, Farah Ouechtati, Houyem Ouragini, Faten Talmoudi, Helmi Guermani, Mohamed Majdi Zorgati. 2 Mourad Mokni, Samir Boubaker, MarieFrançoise Ben Dridi, Koussay Dellagi, Kamel Monastiri, Ahmed Rebai, Fethi Amri, Néji Tebib, Sondes Hadiji, Moez Elloumi, Mounir Frikha, M Hachicha, Tarek Ben Othman, Abdeladhim Ben Abdeladhim.

Mots-clés : Biologie moléculaire, maladies orphelines, Consanguinité.

IP436 / 504. LA MICROFLUIDIQUE, UNE NOUVELLE TECHNOLOGIE POUR L'ANALYSE QUALITATIVE ET QUANTITATIVE DES ACIDES NUCLÉIQUES

D. Mallet (1), C. Lesturgeon (2), L. Michel-Calemard (1), Y. Morel (1), V. Tardy (1)

(I) Hospices Civils de Lyon, Endocrinologie Moléculaire et Maladies Rares, Centre de Biologie et de Pathologie Est, Bron, F69677, France; (2) Caliper Life Sciences, ZAC Paris Nord 2, Roissy CDG, F95911, France

La plateforme de Biologie Moléculaire du Centre de Biologie et Pathologie Est des Hospices Civils de Lyon réalise plus de 60000 réactions de séquences d'ADN par an. La qualité des séquences obtenues est induite par une vérification systématique et une optimisation de chacune des étapes de préparation des réactions de séquençage : $\mathrm{PCR}$ à partir de l'ADN génomique, purification puis normalisation des produits de PCR. Avec l'objectif de rendre l'activité de séquençage plus robuste, plus performante et d'automatiser les étapes en amont, nous avons cherché à améliorer le contrôle qualité des produits de PCR utilisés pour les réactions de séquençage en remplaçant la technique très répandue d'électrophorèse sur gel d'agarose par l'analyse de fragments d'ADN sur l'automate LabChip90 utilisant la technologie de microfluidique «LabChip » développée et commercialisée par Caliper Life Sciences. Cette électrophorèse utilise le Kit « LabChip DNA $5 \mathrm{~K}$ » et permet, en chargeant automatiquement seulement $200 \mathrm{~nL}$ d'échantillon, l'analyse simultanée de la taille en paires de bases et de la concentration en $\mathrm{ng} / \mathrm{microL}$ (limite de détection : $0,25 \mathrm{ng} / \mathrm{microL}$ ) de tous les fragments d'ADN double brin compris entre 50 et 5000 paires de bases contenus dans l'échantillon. Pour adopter cette nouvelle technologie plusieurs paramètres ont été validés : absence de contamination des échantillons chargés automatiquement par le LabChip 90 ; comparaison et corrélation des résultats obtenus sur gels d'agarose et LabChip90; reproductibilité, répétitivité, précision et linéarité des valeurs de concentration et de taille pour des produits de PCR d'environ 370,500, 1400 et 2300 paires de bases largement utilisés dans l'activité de routine de notre laboratoire. Après plusieurs mois d'utilisation, les résultats obtenus sur plus de 5000 échantillons ont démontré que l'utilisation du LabChip90 pour remplacer les gels d'agarose apporte de nombreux avantages sur la qualité des résultats et l'organisation du laboratoire : diminution des risques biologiques pour l'utilisateur et des coûts d'élimination des déchets en supprimant l'utilisation du bromure d'éthidium indispensable à la technique d'électrophorèse sur gel d'agarose ; réduction des temps d'obtention des analyses, avec affichage en temps réel des électrophorèses toutes les 30 secondes et visualisation complète des résultats pour une plaque 96 puits en moins d'une heure ; amélioration de l'appréciation qualitative des résultats de PCR: visualisation des amorces en excès, évaluation de la spécificité permettant une optimisation des conditions de PCR ; quantification précise des produits de PCR purifiés pour normaliser les réactions de séquences; amélioration de la traçabilité, de la sauvegarde et de la gestion des données, avec un transfert sécurisé vers des automates de pipetage, sans nouvelle saisie des identifications des échantillons, s'intégrant dans un processus d'automatisation. Cette étude a montré que le LabChip90 est un outil adapté au contrôle de qualité des produits PCR de notre plateforme de séquençage haut débit mais nécessite une réorganisation du travail pour une utilisation optimale. D'autres applications telles que la recherche de mutations ponctuelles par Restriction Fragment Lenght Polymorphism (RFLP) ou Allele-Specific Oligonucleotide hybridization (ASO) sont en cours de validation.

Mots-clés : analyse qualitative des acides nucléiques, analyse quantitative des acides nucléiques, électrophorèse.

IP437 / 508. MUTATION DU GÈNE LMNB2 DANS UN SYNDROME PROGÉROÏDE AVEC LIPODYSTROPHIE

C. Rouzier (1), V. Esteves-Vieira (1), P. Roll (2), P. Bourgeois (1), A. De Sandre-Giovannoli $(1,3)$, N. Lévy $(1,3)$

(1) Département de génétique médicale, laboratoire de génétique moléculaire-CHU Timone Marseille ; (2) Service de Biologie Cellulaire-CHU La Conception Marseille; (3)Faculté de médecine de Marseille INSERM UMR 910

$M / S$ hors série $n^{\circ} 1$, vol. 24 , janvier 2008 
Introduction : Les laminopathies sont des pathologies dues à des mutations dans les gènes codant pour les protéines de la lamina nucléaire, les lamines, et leurs partenaires fonctionnels. Elles sont caractérisées par un spectre phénotypique très large. À ce jour, on recense une quinzaine de pathologies différentes que l'on peut classer dans quatre groupes principaux : les pathologies du muscle strié, les syndromes lipodystrophiques, les neuropathies périphériques et les syndromes de vieillissement prématuré. La majorité des mutations pathogènes ont été identifiées dans le gène $L M N A$ codant les lamines $\mathrm{A} / \mathrm{C}$. Cependant, des mutations dans les gènes $L M N B 1, E M D$, $L B R, L E M D 3$, codant respectivement pour la lamine B1, l'emerine, le récepteur des lamines B et MAN1 sont également impliquées dans des phénotypes proches ou chevauchants. Récemment, une étude a identifié chez des patients atteints de lipodystrophie partielle acquise (Syndrome de Barraquer-Simons) trois nouveaux variants dans le gène $L M N B 2$. Leur effet pathogène n'a cependant pas été étudié et les auteurs suggèrent plutôt un effet génétique prédisposant dans une pathologie multifactorielle. Le but de notre étude, dans le contexte d'un PHRC national, était d'étendre le spectre phénotypique des laminopathies en identifiant de nouvelles mutations dans des gènes candidats, dont le gène $L M N B 2$, codant les lamines nucléaires B2 et B3. En effet, en plus de leur implication récente dans des cas de lipodystrophie partielle acquise, il a été démontré in vivo dans un modèle murin KO pour lmnbl que les lamines B sont essentielles pour le développement et l'intégrité cellulaire. Matériel et méthode : Nous avons étudié les 12 exons du gène $L M N B 2$ par DHPLC suivi de séquençage chez 60 patients présentant des syndromes progéroïdes segmentaires étiquetés nosologiquement, tels que le syndrome de Werner atypique, ou n'appartenant pas à des catégories nosologiques spécifiques mais porteurs de signes évocateurs de laminopathie systémique. Pour chaque patient, les gènes $L M N A$ et $F A C E 1$ avaient été étudiés au préalable sans mettre en évidence de variation de séquence potentiellement pathogène. Résultats : Nous avons identifié une mutation faux-sens à l'état hétérozygote dans l'exon 8 du gène $L M N B 2, c .1252 \mathrm{C}>\mathrm{T}$ ( $\mathrm{p} . \mathrm{R} 418 \mathrm{~W})$, chez une fillette présentant une lipodystrophie associée à une cataracte, une perte des cheveux, une dysmorphie faciale, des pieds creux et une cytolyse hépatique. L'étude du transcrit par RT-PCR n'a pas permis de détecter d'anomalie d'épissage et a confirmé la mutation p.R418W à l'état hétérozygote. Une prédiction de pathogénicité in silico indiquait « un effet délétère probable». Enfin, cette mutation était absente chez 200 chromosomes étudiés dans une cohorte indépendante de témoins sains. Discussion: À ce jour aucune mutation pathogène n'a été décrite dans le gène $L M N B 2$. Nous décrivons pour la première fois une mutation dans le gène $L M N B 2$ dans un syndrome associant des signes de vieillissement prématuré à une lipodystrophie. Cette mutation est une substitution d'une arginine en tryptophane dans le domaine correspondant au signal de localisation nucléaire, très conservé entre les espèces. Il a été démontré que des mutations dans le domaine fonctionnel correspondant du gène LMNA entraînaient des anomalies d'assemblage des lamines. La localisation de la mutation p.R418W dans un domaine fonctionnel conservé, les résultats obtenus in silico, ainsi que son absence dans un nombre important de chromosomes témoins sont des arguments très en faveur de sa pathogénicité. Pour compléter notre étude des analyses fonctionnelles (Western Blot, immunocytochimie) ainsi qu'une étude familiale sont en cours.

Mots-clés : laminopathies, $L M N B 2$, lipodystrophie.

घP438 / 509. MFN2 EST MUTÉ DANS $11 \%$ DES CAS DE CMT2 : IDENTIFICATION DES ENJEUX DIAGNOSTIQUES SPÉCIFIQUES À PARTIR D'UNE SÉRIE DE 226 PATIENTS NON APPARENTÉS

N. Palot Bonello (1), M. Mayençon (2), R. Bernard (1, 3), C. Pécheux (1), N. Martini (1), K. Amarof (4), J. Pouget (5), S. Attarian (5), P. Latour (2), N. Lévy $(1,3)$

(1) Département de Génétique médicale Laboratoire de Biologie Moléculaire CHU Timone Marseille; (2) Neurogénétique Centre de Biologie Groupement Hospitalier Est CHU Lyon; (3) Inserm UMR 910 Faculté de médecine Marseille ; (4) Unité de neuromyologie Hôpital Pierre ZobdaQuitman CHU Fort de France ; (5) Service des maladies neuromusculaires CHU Timone Marseille

Introduction: La maladie de Charcot-Marie-Tooth (CMT) désigne le groupe des neuropathies périphériques héréditaires sensitives et motrices. Ce groupe de pathologies est caractérisé par son hétérogénéité tant au niveau phénotypique que génotypique. La mise en évidence de mutations dans certains gènes de CMT a permis d'établir certaines corrélations entre le type de CMT et le gène identifié comme muté et responsable de la pathologie. Les mutations dans le gène $M F N 2$ sont responsables de formes autosomiques dominantes de CMT2 (forme axonale) et ont été suspectées dans $20 \%$ de ces cas. Nous avons souhaité explorer les cohortes de patients adressés aux laboratoires de Marseille et de Lyon afin de caractériser ces patients sur le plan moléculaire, d'éprouver et d'optimiser nos stratégies d'exploration moléculaire et d'interpréter les conséquences des variants nouvellement identifiés pour le gène $M F N 2$. Matériels et Méthodes : Nous avons réalisé une étude rétrospective de l'analyse du gène MFN2 sur une cohorte de 226 individus non apparentés suspects de CMT dont 184 individus présentaient une forme familiale de CMT2. L'analyse du gène $M F N 2$ a été réalisée par un criblage initial par DHPLC suivi d'un séquençage des fragments présentant un profil de type hétéroduplex. Résultats : Nous avons mis en évidence des variations de séquence dans le gène $M F N 2$ chez 25 individus dont 23 présentaient une forme dominante de CMT2. Sur ces 25 individus, 24 variations de séquence différentes ont été identifiées dont seulement 8 sont rapportées dans la littérature et les bases de données. Il s'agit de 20 mutations de type faux-sens, 1 non-sens et 3 variations introniques. Notre fréquence de mutations dans cette étude est de $11,1 \%$. Discussion : Des mutations dans le gène $M F N 2$ n'ont été rapportées comme responsables de formes dominantes de CMT2 que depuis 2004. L'exploration moléculaire de ce gène reste donc délicate au niveau de l'interprétation de nouvelles anomalies moléculaires. La mise en évidence d'une variation de séquence non répertoriée chez un individu atteint, pose le problème de sa pathogénicité potentielle mais incertaine. Trancher entre mutation pathogène et polymorphisme rare n'est à ce jour pas possible dans tous les cas, et se pose alors la question de pratiquer ou non les explorations des autres gènes responsables de CMT2, ce qui n'est pas sans conséquences, ni pour les patients, ni en termes de stratégie de laboratoire et de coût. D'autre part si la fréquence des mutations dans ce gène avait initialement été estimée à environ $20 \%$, sur la base des analyses réalisées chez des familles de CMT2 dominant liées au locus 1p36, elle semble être revue à la baisse. Cette discordance peut s'expliquer par le fait que nous avons exploré des familles de taille plus réduite ainsi que des cas sporadiques, en dehors de toute analyse de liaison préalable. Conclusions et perspectives: L'interprétation des anomalies moléculaires non rapportées dans la littérature et les bases de données nécessiterait de pouvoir réaliser plus souvent qu'il n'est possible en pratique une enquête familiale de ségrégation. De plus, des analyses fonctionnelles en RTQ-PCR sont en cours pour les mutations faux-sens ou « à priori silencieuses » ce qui permettrait éventuellement de mettre en évidence des perturbations de l'épissage aussi bien au niveau qualitatif que quantitatif,

Mots-clés : Charcot-Marie-Tooth, MFN2, mutations.

- 9439 / 510. UNE ANALYSE MOLÉCULAIRE SYSTÉMATIQUE DES GENES USH DANS UNE COHORTE DE PATIENTS USHER TYPE I ET II MONTRE L'IMPORTANCE DE L'IMPLICATION DES GËNES MYO7A ET USH2A

M'hamed Grati (1, 2), S. Marlin (1), D. Feldmann (3); L. Jonard (3), C. Koval (3), S. Bahaban (3), A.-F. Grange (3), S. Delmaghani (2), B. Delobel (4), H. Dollfus (5), A. David (6), J. Vigneron (7), D. Bonneau (8), L. Faivre (9), C. Francannet (10), D. Lacombe (11), S. Masmoudi (12), R. Couderc (3), J. Weissenbach (13), C. Petit (2), F. Denoyelle (14) (I) Unité de Génétique Médicale, INSERM U587, Hôpital d'Enfants Armand-Trousseau, AP-HP, Paris, France; (2) Unité de Génétique des Déficits Sensoriels, INSERM U587, Institut Pasteur, Paris, France ; (3) Service de Biochimie et de Biologie Moléculaire, INSERM U587, Hôpital d'Enfants Armand-Trousseau, AP-HP, Paris, France ; (4) Centre de Génétique, Hôpital St-Antoine, Lille, France; (5) Service de Génétique médicale, Hôpital de Hautepierre, Strasbourg, France; (6) Service de Génétique, Hôtel Dieu, Nantes, France; (7) Maternité Régionale Adolphe-Pinard, Nancy, France; (8) Centre de Référence des Maladies Neurogénétiques, Centre Hospitalier Universitaire d'Angers, France ; (9) Unité de Génétique Médicale, Hôpital, Dijon, France; (10) Génétique Médicale, Hôtel-Dieu, Clermont-Ferrand, France; (11) Centre de Génétique, Hôpital Pellegrin, Bordeaux, France; (12) Laboratoire de Génétique Moléculaire Humaine, Sfax, Tunisie; (13) Le Genoscope - Centre National de Séquençage, Évry, France; (14) Service d'ORL et de Chirurgie Cervico-faciale, INSERM U587, Hopital d'Enfants Armand-Trousseau, AP-HP, Université Paris VI, Paris, France

Le syndrome de Usher est une maladie sensorielle rare qui associe une surdité neurosensorielle à une rétinite pigmentaire. Elle se transmet selon un mode autosomique récessif. Le syndrome de Usher est cliniquement et génétiquement hétérogène ( 9 gènes clonés pour 3 sous-types cliniques). Le syndrome de type I (USH1) est le plus sévère et caractérisé par une surdité congénitale profonde bilatérale associée à une aréflexie vestibulaire bilatérale et à une rétinite pigmentaire généralement précoce et sévère. Nous avons recherché des mutations dans les six grands gènes en cause dans les syndromes de Usher de types I et II : MYO7A, USHIC, $P C D H 15$ et $C D H 23$ causant USH1, et USH2A et VLGRI pour USH2, dans une cohorte de 81 
patients, 75 d'origine française, 2 tunisienne et 4 iranienne. Les patients étaient en majorité des cas sporadiques de syndrome de Usher de type I et II. Notre recherche a été fondée sur le séquençage génomique des séquences codantes des six gènes pour tous les patients. Chez ces patients, nous avons identifié 53 variations distinctes potentiellement pathogènes, principalement dans MYO7A ( 38\%) et USH2A ( $37 \%)$, et avec une fréquence moindre dans VLGRI $(\sim 15 \%)$ et PCDH15 $(\sim 8 \%)$. Parmi ces mutations, seulement $\sim 15 \%$ ont été déjà décrites. La plupart des mutations se répartissent aléatoirement dans les gènes. Les nouveaux variants dans MYO7A sont le plus souvent des mutations non-sens $(\mathrm{n}=7)$. Dans USH2A et VLGR1, nous avons détecté plusieurs insertions et délétions d'acides nucléiques qui pourraient conduire à la synthèse de protéines tronquées ( $\mathrm{n}=8$ et 5 , respectivement). Un total de 16 variations faux sens non tolérées ont été trouvées dans MYO7A, USH2A et VLGRI dont la pathogénicité reste à démontrer. Nous avons confirmé l'abondance des mutations p.Asp75fs dans l'exon3 de MYO7A, p.Arg80fs dans l'exon 3 de USHIC et p.Glu767fs dans l'exon 12 de USH2A qu'il serait utile de séquencer systématiquement chez les nouveaux patients. Cependant, le large panel de nouvelles mutations retrouvées dans cette cohorte représente une difficulté à développer une puce de diagnostic génétique chez les patients atteints du syndrome de Usher.

Mots-clés : syndrome de Usher, gènes USH, mutations.

\section{IP440 / 514. SYNDROME CHARGE : UN SITE PRIVILÉGIÉ DE MUTATION DANS L'EXON 8 DU GÈNE $\mathbf{C H D} 7$ ?}

F. Bilan (1), C. Tap (5), C. Sain-Oulhen (3), A.M. Delvaux (1), J. Rivet (1), C. Gohler (3), M. Boissonnot (4), A. Poupeau (1), A. Kitzis (1), B. Gilbert-Dussardier (2)

(1) Laboratoire de Génétique Cellulaire et Moléculaire-CHU de Poitiers ; (2) Service de génétique Médicale, CHU de Poitiers; (3) Service d'ORL, CHU de Poitiers ; Service d'Ophtalmologie, CHU de Poitiers ; (5) CESSA de Larnay-Vienne 86

Introduction : Le syndrome CHARGE est une maladie génétique de type autosomique dominant, généralement sporadique, dont la prévalence varie de1/8 500 à 1/12 000 selon les études. CHARGE est un acronyme anglais désignant les principales manifestations cliniques retrouvées chez les patients souffrant de ce syndrome $(\mathrm{C}$ : Colobome oculaire ; $\mathrm{H}$ : anomalie cardiaques ; A : Atrésie des choanes; R : Retard du développement ; G : hypoplasie Génitale ; E : anomalies de l'oreille externe et/ou interne). Les critères de diagnostic clinique ont été redéfinis par Blake puis Verloes qui propose un système basé sur 3 critères majeurs (règle des $3 \mathrm{C}$ : Colobome, atrésie des Choanes et hypoplasie des Canaux semi-circulaires) et 5 critères mineurs. Un gène responsable de ce syndrome a été identifié par Vissers en 2004 par CGH microarray; il s'agit de $C H D 7$, composé de 38 exons sur une région génomique de $188 \mathrm{kpb}$ qui code pour une protéine de 2997 aa appartenant à la famille des ATPase/Hélicase à chromodomaines. Des anomalies de ce gène ont été retrouvées chez environ $60 \%$ des patients atteints de ce syndrome. Les mutations publiées sont dispersées le long du gène sans accumulation particulière dans un exon ou un domaine particulier de la protéine. Matériel et méthodes : Au CHU de Poitiers, en collaboration avec l'équipe du CESSA (Centre d'Éducation Spécialisée pour Sourds Aveugles), nous avons mené une étude sur 15 patients atteints du syndrome CHARGE sélectionnés selon les critères cliniques définis par Verloes. Afin d'analyser le gène $C H D 7$, nous avons amplifié à l'aide de 40 PCR l'intégralité des régions codantes ainsi que chaque jonction exon/ intron puis analysé chaque fragment PCR par dHPLC. Les réarrangements intragéniques ont été étudiés par la technique de MP/LC (PCR fluorescentes multiplexées semi-quantitatives analysées par dHPLC). Résultats : Nous rapportons la présence d'une mutation récurrente dans l'exon 8 c.2504-2508delATCTT (ou p.Tyr $835 \mathrm{fs} 848 \mathrm{X}$ ) chez deux patients non apparentés. Ces deux patients sont de sexe masculin et âgés de 19 et 27 ans. Ils présentent tous deux une forme sévère du syndrome: surdité de perception bilatérale sévère à profonde (l'agénésie des canaux semi-circulaires n'a pu être documentée que chez l'un des 2), un colobome rétinien (unilatéral pour l'un et bilatéral avec faible acuité visuelle résiduelle pour l'autre), une cardiopathie à révélation néonatale (type tétralogie de Fallot pour l'un et Ebstein pour l'autre), un hypogonadisme avec retard de croissance staturo-pondéral à - 3 DS, retard pubertaire et micropénis, une asymétrie du visage avec paralysie faciale unilatérale, oreilles dysplasiques asymétriques et palais étroit, une scoliose. L'un a présenté des troubles sévères de la déglutition dus à une paralysie laryngée ayant nécessité une gastrostomie jusqu'à l'âge de 4 ans et une trachéotomie jusqu'à l'âge de 16 ans. Il présente en plus des pieds bots. L'atrésie bilatérale des choanes n'était présente que chez l'un, l'autre présentant un simple rétrécissement d'une fosse nasale. L'un peut communiquer par langue des signes et oralisation : l'autre n'a que très peu de communication en raison d'un déficit visuel sévère. Discussion : Exception faite de l'atteinte laryngée chez l'un, ces 2 patients non apparentés présentent une forme clinique similaire en terme de système ou organe atteint, même si le degré d'atteinte au niveau d'un organe peut varier. Des gènes ou des polymorphismes modificateurs pourraient être impliqués. Cette même mutation a été rapportée chez 2 autres patients ce qui suggère qu'il existe au niveau de l'exon 8 de $C H D 7$ des régions sensibles et propices à l'apparition de mutation. L'un de ces patients a une forme sévère du syndrome, comparable à celle de nos 2 patients. Pour l'autre, les informations cliniques ne sont pas disponibles. La présence d'un site privilégié de mutations sur l'exon 8 de $\mathrm{CHD} 7$ doit orienter le choix stratégique d'étude de ce gène.

Mots-clés : CHARGE, diagnostic moléculaire, corrélation génotype/phénotype.

IP441 / 515. HÉMOCHROMATOSE JUVÉNILE TARDIVE ET
HÉTÉROZYGOTIE COMPOSITE: MISE EN EVIDENCE DE
DEUX NOUVELLES MUTATIONS DANS LE GENE CODANT DEUX NOUVELLES

E. Cadet (1), I. Fajardy (2), K. Foulon (1), L. Castera(2), P.M. Danze (2), E. Verlet (3), J. Rochette (1)

(1) Laboratoire de Génétique Moléculaire Médicale, CHU d'Amiens ; (2) Laboratoire de Biochimie, CHRU de Lille; (3) Service de Médecine Interne, $\mathrm{CH}$ de Dunkerque

Introduction: L'hémochromatose de type 2 ou hémochromatose juvénile est une forme sévère de surcharge en fer de transmission autosomique récessive. Elle se caractérise par une apparition précoce des signes cliniques dominés par un tableau d'hypogonadisme et une insuffisance cardiaque avec ou sans troubles du rythme. Il existe une hétérogénéité génétique avec 2 formes notées $2 \mathrm{~A}$ et $2 \mathrm{~B}$ liées respectivement à des mutations dans les gènes codant l'hémojuvéline $(H J V)$ et l'hepcidine $(H A M P)$; la majorité des cas étant due à des mutations du gène $H J V$. Cas clinique : Notre propositus, un homme de 31 ans originaire du Nord de la France, se présente avec une mélanodermie, une asthénie, une cardiomyopathie dilatée avec troubles du rythme ainsi qu'un hypogonadisme hypogonadotrophique. Le bilan ferrique montre une surcharge martiale évidente : ferritine à 6000 microgL-1, coefficient de saturation de la transferrine (CST) à $98 \%$. La biologie hépatique est également perturbée. L'étude par IRM retrouve une importante surcharge hépatique à $300 \mathrm{micromol} \mathrm{de} \mathrm{fer/g} \mathrm{de}$ foie sec $(\mathrm{N}<36)$. Avec les saignées itératives $(60 \mathrm{~g}$ de fer soustrait à ce jour), l'évolution clinique a été satisfaisante : baisse progressive de la ferritine à 20 microgL-1, amélioration très nette de l'état général, normalisation des problèmes cardio-vasculaires et du bilan hépatique. Enquête familiale et moléculaire : Une enquête familiale a été entreprise chez la sæur et les parents du propositus ainsi que chez la grand-mère et les deux oncles côté paternel. Tous, hormis les parents, montrent des signes de surcharge. La sœur ( 25 ans) présente une ferritine à 800 microgL-1 et un CST à $89 \%$. Seule une asthénie est rapportée. Pour les oncles âgés de 52 et 58 ans, l'exploration biochimique montre des valeurs augmentées du CST $(>90 \%)$ et de la ferritinémie (1 630 et 768 microgL-1). À 82 ans, la grand-mère présente un CST à $48 \%$ et une ferritine à 669 microgL-1. Après recueil des consentements écrits, la recherche des mutations C282Y, H63D et S65C du gène HFE a été effectuée par PCR/RFLP. L'étude des autres gènes du métabolisme du fer (HAMP, HJV, RTf2, SLC4OAI) a été réalisée par séquençage. Une simple hétérozygotie H63D est observée chez notre propositus. Aucune anomalie du gène $H F E$ n'est dépistée chez sa sœur. En revanche, l'analyse du gène $H J V$ chez ces deux sujets a permis de révéler l'existence associée de deux nouvelles mutations à l'état hétérozygote. La première, c.581T> $\mathrm{G}$ - exon 3 , est responsable du remplacement d'une Leucine par une Arginine en position 194 de la protéine (L194R). La seconde, c. $1041 \mathrm{C}>\mathrm{A}$ - exon 4 , est une mutation non-sens conduisant à l'apparition prématurée d'un codon stop dans le dernier exon (C347X). Concernant les parents, chacun est porteur à l'état hétérozygote d'une des mutations précédemment citées : L194R pour le père, C347X pour la mère. Une hétérozygotie L194R est également observée chez la grand-mère paternelle qui présente en plus une hétérozygotie composite C282Y/H63D. Pour les oncles paternels, l'analyse a mis en évidence une homozygotie C282Y. Discussion et conclusion: Deux traits phénotypiques sont à souligner dans cette famille : un trait classique d'hémochromatose génétique et un autre d'hémochromatose juvénile expliquée par la présence concomitante de deux mutations non encore décrites. Comparées à d'autres cas déjà rapportés d'hémochromatose juvénile, les manifestations cliniques sont assez tardives. Ce phénotype pourrait d'une part s'expliquer par la position de la mutation $\mathrm{C} 347 \mathrm{X}$. En effet, si l'on prend l'exemple du gène bêta globine, des mutations non-sens présentes dans le dernier exon de celui-ci conduisent à un phénotype d'anémie modérée lié à la présence de taux substantiels d'ARNm. D'autre part, l'homozygotie L194R a été 
associée à des phénotypes tardifs d'hémochromatose juvénile (communication personnelle). Ces résultats illustrent de nouveau l'hétérogénéité des mutations observées dans l'hémochromatose $2 \mathrm{~A}$.

Mots-clés: hémochromatose juvénile, hétérozygotie composite, hémojuvéline.

\section{aP442 / 516. LA COMBINAISON DE POLYMORPHISMES GÉNÉ- TIQUES MODIFIERAIT LE RISQUE DE CANCER DU SEIN ET DE L'OVAIRE}

L. Delort $(1,2)$, N. Chalabi $(1,2)$, S. Satih $(1,2)$, N. Rabiau $(1,2), R$. Bosviel (1, 2), Y.-J. Bignon (1, 2, 3), D. Bernard-Gallon $(1,2)$

(I) Département d'Oncogénétique, Centre Jean-Perrin, CBRV, 28 place Henri-Dunant, BP 38, 63000 Clermont-Ferrand cedex 01; (2) CRNH, 58 rue Montalembert, 63000 Clermont-Ferrand cedex 01; (3) Universite d'Auvergne, 28 place Henri-Dunant, BP 38, 63000 Clermont-Ferrand cedex 01

Les taux d'incidence des cancers du sein et de l'ovaire sont en constante augmentation dans le monde. L'étiologie de ces cancers s'expliquerait en grande partie par la présence de polymorphismes génétiques à l'intérieur de gènes impliqués dans des voies biologiques majeures, gènes qui auraient également la capacité d'interagir avec les facteurs environnementaux et le mode de vie des individus. Notre objectif a été de mener une étude épidémiologique cas-témoins constituée de 934 femmes ayant développé un cancer du sein, 54 femmes ayant développé un cancer des ovaires et de 1000 femmes témoins, dans le but de rechercher les facteurs de risque et de protection génétiques et environnementaux de ces pathologies. Le génotypage de l'ensemble de la population a été effectué pour onze polymorphismes dans sept gènes de faible pénétrance, impliqués dans les métabolismes des xénobiotiques et des œstrogènes (CYPIAI, CYPIBI, NAT2, GSTP1, COMT, ESR, PGR). L'étude des polymorphismes grâce à l'utilisation d'un modèle multigénique a révélé le rôle du gène COMT dans la cancérogenèse mammaire. $C O M T$ semble interagir avec les gènes $C Y P I B I$, $E S R, G S T P 1$ et NAT2 en modifiant le risque ainsi qu'avec le facteur tour de taille/tour de hanche. La présence de polymorphismes dans ces gènes va modifier l'élimination et par conséquent l'exposition de l'organisme aux carcinogènes exogènes et endogènes et bouleverser la susceptibilité de chaque individu face au cancer mammaire. Un rôle plus limité des gènes étudiés a été observé dans l'étude des facteurs de risque des cancers de l'ovaire. La détermination des interactions gène-gène et gène-environnement pourra permettre la mise en place d'une prévention individualisée en identifiant les individus à plus haut risque de cancer.

Mots-clés : cancer du sein et de l'ovaire, polymorphismes génétiques, risque de cancer.

\section{EP443 / 518. CANCER DE LA VESSIE : ÉTUDES DES POLYMOR-} PHISMES GÉNÉTIQUES

L. Delort $(1,2,3)$, L. Joumard (1, 2, 3), N. Chalabi $(1,2,3)$, N. Rabiau $(1,2,3)$, S. Satih (1, 2, 3), R. Bosviel $(1,2,3)$, L. Guy $(1,4)$, J.P. Boiteux $(4,5)$, Y.J. Bignon $(1,2,3,5)$, A. Chamoux $(5,6)$, L. Fontana $(5,6)$, D.J. Bernard-Gallon $(1,2,3)$

(1) Département d'Oncogénétique, Centre Jean-Perrin, CBRV, 28 place Henri-Dunant, BP 38, 63000 Clermont-Ferrand cedex 01; (2) CRNH, 58 rue Montalembert, 63009 Clermont-Ferrand Cedex 01, France; (3) INSERM UMR 484, rue Montalembert, 63005 Clermont-Ferrand Cedex 01 : (4) CHU Clermont-Ferrand, Service d'Urologie, Hôpital G.-Montpied, 58 rue Montalembert, 63003 Clermont-Ferrand Cedex 01 ; (5) Univ. Clermont 1, 28 place Henri-Dunant, 63001 Clermont-Ferrand Cedex 01 ; (6) CHU Clermont-Ferrand, service de Médecine du Travail et des Pathologies Professionnelles, 28 place Henri Dunant, BP 38, 63001 ClermontFerrand Cedex 01

Le cancer de la vessie, sixième rang des cancers en France, fait partie des cancers les plus fréquents. Hormis les rares cas avec une prédisposition génétique et une pénétrance élevée, cette pathologie s'exprime sur un mode multifactoriel. En effet, des polymorphismes dans des gènes de faible pénétrance, c'est-à-dire des gènes en interaction avec les facteurs environnementaux, auraient un rôle dans le développement de cette maladie. Dans ce cas on parle de cancers de la vessie sporadiques. Les gènes les plus étudiés sont ceux qui codent pour les enzymes jouant un rôle dans la détoxification des carcinogènes. La présence de polymorphismes dans ces gènes peut être à l'origine d'une altération fonctionnelle des enzymes et ainsi favoriserait le processus de cancérogenèse. Une étude cas-témoins constituée de 51 cas de cancer de la vessie et 45 témoins a été réalisée. 11 Single Nucleotide polymorphisms (SNP) dans quatre gènes (CYPIA1, CYPIBI, COMT, GSTPI et NAT2) ont été analysés par la technique des sondes MGB (Minor Groove Binder) qui consiste en l'utilisation de la discrimination allélique par la méthode Taqman. Cette étude a permis de mettre en évidence une augmentation du risque de cancer de la vessie en présence des deux polymorphismes étudiés sur le gène GSTPI et sur NAT2-590. En revanche, une diminution du risque est observée pour le SNP NAT2-282. De plus, il est prouvé que le tabac est un facteur de risque de ce cancer mais aucune association n'a été établie entre les polymorphismes étudiés et la consommation tabagique. De ces résultats, seulement des tendances sont données. Pour confirmer, il est nécessaire d'élargir notre étude à un plus grand nombre de cas.

Mots-clés : cancer de la vessie, polymorphismes génétiques, risque de cancer.

IP444 / 521. ANALYSE PROSPECTIVE DE VHL DANS 102 CARCINOMES À CELLULES RÉNALES SPORADIQUES DE L'ADULTE : CORRÉLATIONS AVEC LES FACTEURS HISTOPRONOSTIQUES ET LA SURVIE

P. Fergelot (1, 2), N. Rioux-Leclercq (1, 3), S. Zerrouki (2), F. Jouan (1), N. Collet (1), C. Dubourg (1, 2), M.-C. Malinge (4), D. Masson (1), C. Bendavid (1, 2), M. Denis (1, 2), J.-J. Patard (1, 5)

(1) Institut de génétique et développement de Rennes, Université de Rennes I; (2) Département de biochimie et génétique moléculaire, $\mathrm{CHU}$ de Rennes; ( 3 )Département d'anatomie et cytologie pathologiques, $\mathrm{CHU}$ de Rennes; (4) Service de Génétique, CHU d'Angers ; (5) Service d'urologie, $\mathrm{CHU}$ de Rennes

Introduction : Le cancer du rein représente $3 \%$ des tumeurs malignes de l'adulte et son incidence augmente régulièrement depuis trente ans. D'emblée métastatique dans $30 \%$ des cas, ces tumeurs sont radio et chimiorésistantes. Les formes les plus fréquentes, les carcinomes à cellules rénales de type conventionnel ou à cellules claires (CCRC, $75 \%$ des cancers), se caractérisent par des lésions potentiellement inactivatrices du gène VHL dans plus de la moitié des cas. Les avancées majeures des dernières années tiennent à la description de l'axe $\mathrm{VHL} / \mathrm{HIF} / g e ̀ n e s$ induits par l'hypoxie et à l'introduction des molécules anti-angiogéniques dans l'arsenal thérapeutique. À l'heure actuelle, il est primordial de trouver des marqueurs prédictifs de survie et de réponse à ces nouveaux traitements. Dans cette optique, les données concernant VHL sont contradictoires, du fait de l'hétérogénéité des populations étudiées et des techniques employées. Objectifs : Déterminer le statut VHL des tumeurs opérées au CHU de Rennes et établir des corrélations avec les facteurs histopronostiques et la survie des patients. Matériels et méthodes : Cent deux patients porteurs d'un CCRC opéré entre fin 2002 et fin 2005 ont été inclus dans l'étude. Les tumeurs ont été collectées de façon prospective au Centre de Ressources Biologiques Humaines de Rennes. Les mutations de $V H L$ ont été recherchées sur les 3 exons et les séquences introniques flanquantes par dHPLC (denaturing HPLC), suivie du séquençage de l'exon positif. Les délétions ont été étudiées par MLPA (Methylation Specific Multiplex Ligation-dependent Probe Amplification) et l'hyperméthylation du promoteur du gène a été recherchée par la technique de MS-MLPA (methylation specific MLPA). En parallèle l'expression de la proteine a été détectée par immuno-histochimie sur coupes paraffine. Les résultats ont été comparés aux données cliniques et histologiques réunies dans une base de données dédiée. Résultats : Parmi les 102 patients $34 \%$ présentaient des métastases et/ou une extension ganglionnaire. Soixante-dix mutations ( $68 \%$ des tumeurs), dont 52 de type «perte de fonction », 15 hyperméthylations et 76 délétions ont été retrouvées. Une tumeur présentait les 3 anomalies et 70 tumeurs $(68 \%)$ en présentaient 2 , associant mutation et délétion dans 56 cas et hyperméthylation et délétion dans 14 cas ; 22 tumeurs ne présentaient qu'une seule altération et seulement 10 cas ne montraient aucune anomalie. Parmi ces 32 tumeurs, la protéine était détectée dans $2 / 3$ étaient concordants avec la détection de la protéine. De plus, l'absence d'anomalie du gène $V H L$ associée à la détection de pVHL était corrélée à une survie plus courte (survie sans progression et survie spécifique). Conclusion et perspectives : La stratégie utilisée pour notre étude nous a permis de confirmer la fréquence des altérations de VHL obtenues dans d'autres séries. L'association d'un pronostic très sévère à l'absence d'altération du gène $V H L$ suggère d'autres voies de progression, (voire un nouveau cadre nosologique). La valeur prédictive du statut du gène $V H L$ va être plus largement étudiée par notre équipe au cours du programme de l'INCA « PNES rein » chez des patients traités par les drogues anti-angiogéniques (Sutent ${ }^{\circ}$, Pfizer, Nevaxar ${ }^{\circ}$, Bayer).

Mots-clés : carcinome à cellules rénales, VHL, marqueur moléculaire.

IP445 / 523. L'ÉTUDE MOLÉCULAIRE DU GÈNE WFSI EST INDIQUÉE DANS LES SURDITÉS NON SYNDROMIQUES AVEC COURBE AUDIOMÉTRIQUE ASCENDANTE OU EN U

L. Jonard (1), D. Feldmann (1), S. Marlin (2), C. Koval (1), M. Louha (1), K. Olszewski (1), R. Couderc (1), H. Dollfus (3), V. Drouin-Garraud (4), 
D. Lacombe (5), F. Fellmann (6), C. Francannet (7), G. Lina-Granade (8), C. Petit (9), F. Denoyelle (10)

(1) Laboratoire de Biochimie, Inserm U587, Hôpital Armand-Trousseau, AP-HP, Paris, France; (2) Unité de Génétique Médicale, Inserm U587, Hôpital Armand-Trousseau, AP-HP, Paris, France ; (3) Service de Génétique Médicale, Hôpital de Hautepierre, Strasbourg, France ; (4) Unité de Génétique Clinique, Hôpital Charles-Nicolle, Rouen, France ; (5) Service de Génétique, Hôpital Pellegrin, Bordeaux, France ; (6) Service de Génétique, Hôpital Saint-Jacques, Besançon, France ; (7) Génétique Médicale, Hôtel-Dieu, Clermont-Ferrand, France; (8) Service ORL, Hôpital Edouard Herriot, Lyon, France ; (9) Inserm U587, Unité de Génétique des Déficits Sensoriels, Institut Pasteur, Paris, France; (10) Service d\&\#8217; ORL et de Chirurgie Cervico-Faciale, Inserm U587, Hôpital Armand-Trousseau, AP-HP, Paris, France

Introduction: Le syndrome de Wolfram ou DIDMOAD (pour Diabetes Insipidus, Diabetes Mellitus, Optic Atrophy and Deafness) est une surdité syndromique de transmission autosomique récessive causée par des mutations du gène WFS1. Parallèlement à son implication dans le déterminisme du syndrome de Wolfram, des mutations dominantes de WFS1, siégeant principalement dans l'exon 8 du gène et affectant l'extrémité C-terminale de la protéine, wolframine, sont retrouvées dans des formes non syndromiques de surdité avec atteinte préférentielle des fréquences graves (surdité DFNA6/14/38). Méthodes : Nous avons analysé l'exon 8 du gène WFS1 dans une cohorte de 64 cas sporadiques ou familiaux de surdité non syndromique dont 23 avec courbe audiométrique ascendante (atteinte préférentielle des fréquences graves) et 41 avec courbe en U. Résultats : Une mutation hétérozygote a été identifiée dans 4 cas familiaux : c.923C $>\mathrm{G}$ (p.Ser308Cys), c.1079G >A (p.Cys360Tyr), c.2141A $>$ G (p.Asn714Ser) et c.2421C $>$ A (p.Ser807Arg). En effet, ces variations du gène WFS1, non décrites à ce jour, (i), ségrègent parfaitement avec la surdité, (ii), sont des faux-sens affectant des résidus très conservés de la protéine et décrits comme pathogènes par des modèles prédictifs in silico et (iii), n'ont pas été retrouvées sur 100 chromosomes témoins. Ces 4 cas représentent des formes dominantes multigénérationnelles (jusqu'à 5 générations), une même mutation ségrégeant avec la surdité pouvant, au sein d'une même famille, rendre compte d'une perte auditive avec courbe audiométrique de forme ascendante, ou en U. De plus, des cas d'évolution d'une courbe ascendante vers une courbe en U chez un même individu porteur de la mutation ont été documentés. Conclusion : L'étude moléculaire du gène du syndrome de Wolfram, WFSI, trouve sa pertinence dans le diagnostic génétique des surdités non syndromiques, sporadiques ou familiales, avec courbe audiométrique ascendante ou en U.

Mots-clés: surdité non syndromique dominante, WFS1, courbe audiométrique.

\section{P446 / 530. ÉTUDE DES MUTATIONS DU GÈNE VHL SUR TISSU INCLUS EN PARAFFINE DANS LE CANCER RÉNAL SPORA- DIQUE DE L'ADULTE: COMPARAISON AVEC LE TISSU CONGELÉ}

G. Verhoest (1), J.-J. Patard (1, 2), F. Jouan (1), S. Zerrouki $(1,3)$, S. Dréano (1), S. Mottier (1), M. Denis (1, 3), N. Rioux-Leclercq $(1,4)$, P. Fergelot $(1,3)$

(1) Institut de génétique et développement de Rennes, Université de Rennes-1; (2) Département de biochimie et génétique moléculaire, $\mathrm{CHU}$ de Rennes ; (3) Département d'anatomie et cytologie pathologiques, $\mathrm{CHU}$ de Rennes; (2) Service d'urologie, CHU de Rennes

Introduction : Le cancer du rein représente $3 \%$ des tumeurs malignes de l'adulte. D'emblée métastatique dans $30 \%$ des cas, son incidence augmente régulièrement depuis trente ans. Les formes les plus fréquentes, les carcinomes à cellules rénales de type conventionnel ou à cellules claires (CCRC, $75 \%$ des tumeurs) se caractérisent par des lésions potentiellement inactivatrices du gène $V H L$ dans plus de la moitié des cas. L'introduction des molécules anti-angiogéniques dans l'arsenal thérapeutique nécessite de définir des facteurs prédictifs de survie et de réponse à ces nouveaux traitements. Les données sur la valeur prédictive de VHL sont contradictoires, du fait de l'hétérogénéité des populations étudiées et des techniques employées. La fréquence des mutations retrouvées varie également en fonction du type de tissu étudié, allant de $29 \%$ à partir du tissu inclus en paraffine à $71 \%$ à partir du tissu congelé. Objectif : Comparer les mutations du gène $V H L$ retrouvées dans les tumeurs incluses en paraffine aux mutations mises en évidence sur tissu congelé, afin d'évaluer la fiabilité de l'analyse ADN sur tissus fixés pour son application en pratique courante. Matériel et Méthodes : 76 patients opérés d'un CRCC entre 2002 et 2005 au CHU de Rennes, et pour lesquels une analyse du gène VHL sur tissu congelé avait été réalisée, ont été inclus : 73 tumeurs étaient mutées et 3 non mutées. L'ADN extrait des blocs de paraffine (kit RecoverAll(tm),
Ambion) a été soumis à une électrophorèse en gel d'agarose afin d'évaluer sa qualité. Une double amplification par PCR a été réalisée. Les échantillons amplifiés étaient ensuite séquencés sur ABI PRISM 3130 Genetic Analyser. Les résultats du séquençage étaient comparés à ceux obtenus à partir du tissu congelé, ainsi qu'aux données clinico-biologiques contenues dans une base de données. Résultats: L'ADN a pu être extrait des 76 tumeurs. L'ADN était très dégradé dans $34,2 \%$ des cas, avec un taux d'amplification significativement inférieur. Parmi ces ADN très dégradés, $57,7 \%$ n'étaient pas séquençables et $42,3 \%$ étaient discordants avec le tissu congelé (mutation non retrouvée et/ou néo-mutation). Soixante-douze pour cent des échantillons étaient séquençables, mettant ainsi en évidence un taux de mutation global sur tissu paraffine de $52,1 \%$ (38/73). Si l'on ne considérait que les échantillons séquençables, ce taux était de $69,1 \%$. Seulement la moitié de ces mutations étaient concordantes avec le congelé. Sont apparues $10,5 \%(8 / 76)$ de néo-mutations de type faux sens $(40 \%)$ ou silencieuses $(60 \%)$, et $28,9 \%$ (22/76) des mutations connues en congelé avaient disparu. Quarante pour cent des mutations mises en évidence sur tissus fixés étaient de type faux sens, alors que ce sont les insertions/ délétions avec décalage du cadre de lecture qui prédominaient en tissu congelé $(42,7 \%)$. Parmi les facteurs cliniques et biologiques analysés, seule la qualité de l'ADN extrait influençait de manière significative l'amplification en PCR et le séquençage. Conclusion : L'analyse de l'ADN tumoral à partir des tissus inclus en paraffine permet la mise en évidence de mutations au niveau du gène $V H L$, mais avec une sensibilité moindre que pour les tissus congelés. De plus, dans cette étude un tiers des cas ne concordait pas avec le tissu congelé.

Mots-clés : VHL, carcinome à cellules rénales, mode de conservation.

DP47 / 531. CARACTÉRISATION D'UNE DÉLÉTION GERMI-
NALE DE 7,6 MB ASSOCIÉE À UNE FORME SÉVĖRE DE SYN-
DROME NEUROFIBROMATOSE-NOONAN ET COMPRENANT
LE GENNE NFI AINSI QU'UNE CENTAINE D'AUTRES GÈNES
TE GENE NFI AINSI QU'UNE CENTAINE D'AUTRES GENES É. Pasmant (1), A. Dusordet (2), A. Coeslier-Dieux (3), I. Laurendeau (1), B. Parfait (1), M. Vidaud (1, 2), D. Vidaud $(1,2)$, I. Bièche $(1,4)$ (1) UMR745 INSERM, Faculté des Sciences Pharmaceutiques et Biologiques Paris-Descartes, 4 avenue de l'Observatoire, 75006, Paris, France ; (2) Service de Biochimie et de Génétique Moléculaire, Hôpital Beaujon AP-HP, 100 Boulevard du Général Leclerc, 92110, Clichy, France; (3) Service de Génétique Clinique, Hôpital Jeanne de Flandre, Avenue Oscar Lambret, 59037 Lille, France +(33)(0)320445962; (4) Laboratoire d'Oncogénétique, INSERM U735, Centre René-Huguenin, St-Cloud, France

Une grande délétion germinale du locus NF1 a été mise en évidence par l'hémizygotie de marqueurs microsatellites chez une patiente atteinte d'une forme sévère du syndrome Neurofibromatose-Noonan (NFNS). Le propositus, une jeune fille de 8 ans, présente à la fois des symptômes de neurofibromatose de type 1 (NF1) et de syndrome de Noonan (NS). La présente étude a pour objectifs de cloner précisément les points de cassure de cette grande délétion et d'évaluer le rôle des gènes délétés dans ce phénotype particulièrement sévère de NFNS. Une technique de cartographie par dosage génique en PCR temps réel de 45 STS (sequence tagged site) suivie d'une XL-PCR et d'un séquençage permis de caractériser les points de cassure. La délétion mesure exactement $7.586 .986 \mathrm{pb}$ et est située en $17 \mathrm{q}$ entre les nucléotides 24.405 .358 et 31.992.345 (NCBI Build 36.1). Elle comprend l'ensemble du locus NF1 ainsi qu'une centaine d'autres gènes parmi lesquels des nombreuses chémokines (CCL1, 2, 3, 5, 7, 8, 11, 12, $13,15,16,18$ et 23 ) et 4 gènes de microRNAs (has-mir-423, -193a, -365-2 et -632). Il est à noter que les points de cassure sont situés dans 2 éléments de type SINE. Le point de cassure centromérique est localisé dans l'intron 4 du gène PIPOX (pipecolic acid oxidase NM_016518) et le point de cassure télomérique dans l'exon 5 du gène ZNF403 (zinc finger protein 403 NM_024835 aussi dénommé LCRG1: Laryngeal carcinoma related gene) codant un facteur de transcription. Comme PIPOX et ZNF403 présentent la même orientation transcriptionnelle, nous avons fait l'hypothèse et démontré l'existence d'un transcrit chimérique. Les contributions respectives au phénotype particulièrement sévère de NFNS, des nombreux gènes délétés (outre NFI) et du transcrit chimérique et/ou d'une possible protéine de fusion restent à explorer.

Mots-clés : syndrome neurofibromatose-Noonan, délétion, transcrit chimérique.

P448 / 542. ANALYSE MUTATIONNELLE DU GÈNE DE LA DYSFERLINE DANS UNE GRANDE COHORTE DE PATIENTS : IDENTIFICATION DE STRATÉGIES THÉRAPEUTIQUES CIBLÉES

M. Krahn $(1,2,3)$, N. Wein $(1,2)$, M. Bartoli $(3), K$. Nguyen $(1,2)$, V. Labelle (1), A. Borges (1), S. Courrier (4), G. Bassez (5), 
D. Figarella-Branger (6), J. Pouget (7), E. Salvo (1), H. Hammouda (8), B. Eymard (5), J.A. Urtizberea (9), P. Cau (2), I. Richard (3), C. Béroud (10), F. Leturcq (11), N. Lévy $(1,2)$, et le Réseau Français d'étude des Dystrophies musculaires de Ceintures

(I) Département de Génétique Médicale, Hôpital d'enfants de la Timone, Marseille, France; (2) INSERM U910, "Génétique médicale et génomique fonctionnelle ", Faculté de Médecine de Marseille, France; (3) Genethon, CNRS FRE 3018, Evry, France ;(4) Centre d'Enseignement et de Recherche en Génétique médicale, Faculté de Médecine de Marseille, France; (5) Institut de Myologie, Groupe Hospitalier Pitié-Salpêtrière, Paris, France; (6) Laboratoire d'Anatomopathologie, Hôpital Timone, Marseille, France ; (7) Service de Neurologie, Hôpital Timone, Marseille, France ; (8) AFM et Généthon, Evry, France ; (9) Hôpital Marin, APHP, Hendaye, France; (10) Laboratoire de Génétique Moléculaire, Institut de Génétique Humaine, Montpellier, France; (11) Laboratoire de Biochimie Génétique, Hôpital Cochin, Paris, France

Introduction : Les mutations dans le gène codant la dysferline (DYSF) causent différents phénotypes, principalement la dystrophie musculaire des ceintures de type2B (Limb Girdle Muscular Dystrophy type 2B,LGMD2B) et la myopathie de Miyoshi (MM), de transmission autosomique récessive. En raison de l'hétérogénéité clinique, une analyse protéique initiale de la dysferline sur échantillons de biopsie musculaire, est nécessaire pour orienter le diagnostic. Le diagnostic de certitude est basé sur l'analyse mutationnelle du gène DYSF, qui est rendue difficile en routine hospitalière par la grande taille du gène DYSF (> $150 \mathrm{kbp}, 55$ exons). Les méthodes de précriblage mutationnel sont par conséquent des outils de choix. Patients et Méthodes : 135 patients présentant un déficit en dysferline identifié sur biopsie musculaire ont été inclus pour analyse du gène $D Y S F$. Le criblage mutationnel a été effectué dans sur le plan génomique par DHPLC, suivi du séquençage direct des variants identifiés. Résultats et Discussion : Pour 102 patients $(75 \%)$ deux mutations constitutionnelles délétères ont été identifiées, permettant une confirmation du diagnostic sur le plan génétique. Un seul allèle délétère a été identifié chez 27 patients $(20 \%)$. Nous développons actuellement des analyses complémentaires, qui seront indiquées en cas d'identification d'un seul, ou d'aucun allèle pathogène après criblage mutationnel génomique. Parmi les mutations identifiées, 37 n'ont pas été rapportées dans la littérature, ce qui correspond à plus de $10 \%$ des mutations identifiées dans le gène $D Y S F$ à ce jour. Il s'agit principalement de mutations faux-sens, ou de mutations causant un décalage du cadre de lecture, mais nous rapportons également une proportion important de mutations non-sens en comparaison à d'autres séries. Nous avons crée une base de données locus-spécifique, UMD-DYSF (Universal Mutation Database for Dysferlin), dans laquelle sont saisies toutes les données cliniques et moléculaires de notre série, et de la littérature. Un avantage majeur de cette base de données est l'intégration d'outils d'analyse bioinformatique, permettant d'effectuer des analyses statistiques, et d'évaluer la pathogénicité de variants nouvellement identifiés (faux-sens, isosémantiques ou introniques) ce qui en fait un outil précieux d'aide au diagnostic moléculaire. Cette base de données est par ailleurs un outil central pour déterminer des approches thérapeutiques ciblées. En effet, la grande taille du gène et son large spectre mutationnel ne permettent pas d'envisager une approche thérapeutique unique. L'interrogation de la base UMD-DYSF permet de déterminer des groupes de mutations candidates à une approche thérapeutique donnée. La proportion importante de mutations non-sens (environ 20\%) permet par exemple d'envisager les stratégies de translecture de codons stop, ou d'exonskipping. Nous illustrons par un cas particulier l'identification d'approches thérapeutiques éventuelles à partir des données mutationnelles de patients : chez une patiente présentant un phénotype atypique/modéré de dysferlinopathie, nous avons caractérisé la première grande délétion génomique décrite dans le gène DYSF. Cette délétion multi-exonique homozygote permet néanmoins le maintien de certains domaines essentiels de la protéine, conduisant à une molécule «mini-dysferline». Les monocytes exprimant en condition physiologique la dysferline, nous avons utilisé ce modèle cellulaire humain pour étudier la protéine tronquée. D'autre part, la taille du transcrit délété étant compatible avec un transfert génique efficace, par vecteur dérivé de virus adéno-associé (A.A.V.), nous évaluons actuellement la fonctionnalité de cette « mini-dysferline » dans un modèle murin déficient en dysferline. Conclusion: Nous rapportons la plus grande cohorte d'analyse mutationnelle du gène $D Y S F$ décrite à ce jour. Les données mutationnelles sont analysées par la base UMD-DYSF pour développer des stratégies thérapeutiques ciblées.

Mots-clés : LGMD, dysferline, thérapeutique.

口P449 / 544. ÉTUDE DU RÔLE DU GÈNE SPRED1 CHEZ DES PATIENTS PRÉSENTANT UN PHÉNOTYPE APPARENTÉ À LA NEUROFIBROMATOSE DE TYPE 1

E. Pasmant (1, 2), A. Sabbagh (1, 2), J. Masliah (2), B. Parfait (1, 2), P. Wolkenstein (3), D. Vidaud $(1,2), M$. Vidaud $(1,2)$, Réseau NF France
(1) UMR745 INSERM, Faculté des Sciences Pharmaceutiques et Biologiques Paris-Descartes, 4 av. de l'Observatoire, 75006, Paris, France; (2) Service de Biochimie et de Génétique Moléculaire, Hôpital Beaujon AP-HP, 100 Bd du Gal Leclerc, 92110, Clichy, France; (3) Service de Dermatologie, Hôpital Henri-Mondor-APHP, 51 av. du Mal-de-Lattre-deTassigny, 94000 Créteil, France

Des mutations germinales de gènes codant des protéines de la voie RAS MAPK (mitogen-activated protein kinase) ont été récemment identifiées dans plusieurs syndromes héréditaires aux phénotypes chevauchants transmis sur un mode autosomique dominant chez l'homme (Bentires-Alj et al., 2006). Cette découverte a permis l'identification d'un mécanisme unificateur, la dérégulation de la voie RAS MAPK, à l'origine des syndromes neuro-cardiofacio-cutanés (NCFC) qui regroupent : la neurofibromatose de type 1 (NF1, MIM \#162200), le syndrome de Noonan (NS, MIM \#163950), le syndrome LEOPARD (LS, MIM \#151100), le syndrome cardio-facio-cutané (CFCS, MIM \#115150) et le syndrome de Costello (CS, MIM \#218040). Une étude récente décrit des mutations du gène SPREDI (sprouty-related, EVH1 domain containing 1) chez des patients diagnostiqués NF1 selon les critères du NIH mais présentant un phénotype particulier (présence de multiples taches café au lait et de lentigines mais absence de neurofibromes, de nodules de Lisch) et pour lesquels aucune mutation de NF1 n'était retrouvée (Brems et al., 2007). La présente étude a pour objectif de confirmer l'implication du gène SPREDI chez 12 patients présentant ce phénotype particulier et pour lesquels la recherche de mutations du gène NF1 avait été négative. Deux mutations du gène $S P R E D l$ ont été identifiées après séquençage des régions codantes : une mutation non sens déjà connue (190C $>$ T R64X) et une mutation non sens jamais décrite (637C $>$ T Q213X). Nos résultats confirment l'implication du gène SPREDI qui code une protéine de régulation négative de la voie RAS MAPK, dans un nouveau syndrome NCFC. Il apparait maintenant nécessaire de préciser la fréquence des mutations et le spectre phénotypique de ce nouveau syndrome ainsi que l'existence d'éventuelles complications. Les critères diagnostiques de NF1 établis par le NIH doivent prendre en compte ces nouvelles données afin de permettre la mise en place d'un conseil génétique spécifique et adapté, différent de celui de la NF1.

Mots-clés : neurofibromatose de type 1, SPRED1, conseil génétique.

\section{שP450 / 547. DÉTECTION RAPIDE DES MUTATIONS DU GÈNE CFTR PAR ANALYSE DE COURBES DE FUSION HAUTE RÉSOLUTION}

M.P. Audrezet, A. D'abricot, C. Le Marechal, C. Ferec

Laboratoire de Génétique Moléculaire CHU, INSERM U613, Brest, France

La mucoviscidose est la maladie génétique grave de l'enfant la plus fréquente dans les populations d'origine caucasienne. Le gène responsable, appelé CFTR (Cystic Fibrosis Transmembrane conductance Regulator) a été cloné en 1989. Il est composé de 27 exons et code protéine transmembranaire. La mutation majoritaire, une délétion de 3 nucléotides dans l'exon 10 du gène, aboutissant à la perte d'une phénylalanine en position 508 de la protéine, représente environ $70 \%$ des allèles $\mathrm{CF}$, mais ce pourcentage varie en fonction de l'origine géographico-ethnique des patients. 18 ans après la découverte du gène, plus de 1500 anomalies différentes ont été rapportées au sein du Consortium International d'Etudes des mutations du gène CFTR, la majorité d'entre elles étant des mutations ponctuelles ou de courtes insertions-délétions. La connaissance précise des mutations en cause a des implications importantes pour le diagnostic prénatal, pour le dépistage en cascade dans les familles ainsi que pour la compréhension des relations génotype-phénotype.De nombreux kits permettent la détection des mutations les plus fréquentes du gène, mais dans certaines situations, telles que la recherche de mutations rares chez des individus atteints, l'étude chez les conjoints de patients atteints de mucoviscidose, ou le repérage pendant la grossesse d'un intestin hyperéchogène chez le fœtus..., il est parfois nécessaire d'analyser rapidement la totalité de la séquence codante du gène. Pour cela les laboratoires doivent avoir recours à des techniques rapides, efficaces et économiques. C'est pourquoi nous avons choisi de développer une technique d'étude des 27 exons du gène CFTR par l'analyse de courbes de fusion haute résolution sur plateforme Lightscanner ${ }^{\circ} .30$ couples d'amorces ont été choisis et modifiés, en fonction des courbes de fusion des fragments à analyser de façon à amplifier dans les mêmes conditions les 27 exons du gène ainsi que les jonctions introns-exons. Les méthodes d'analyse de chaque amplicon ont été mises au point à partir de témoins exempts de mutations et validées à partir d'échantillons porteurs de plus de 200 anomalies différentes. En conclusion, cette technique est simple, rapide, efficace, économique et automatisable pour une utilisation en routine pour le scanning du gène CFTR.

Mots-clés : CFTR, HRM, mucoviscidose. 

GP51 / 561. GESTION DU LABORATOIRE DE GÉNÉTIQUE
SOUS FILEMAKER PRO

A. Lauge, V. Caux-Moncoutier, C. Dehainault, C. Dubois D'Enghien, D. Michaux, S. Pagès-Berhouet, C. Houdayer, D. Stoppa-Lyonnet Génétique oncologique, Institut Curie, Paris, France

On assiste aujourd'hui à une augmentation considérable de l'activité de nombreux laboratoires de Génétique. Le développement des techniques et l'automatisation permettent de répondre en partie à cet accroissement d'activité mais la gestion de ces nouveaux flux de données ne peut être réalisée sans le développement d'un système informatique dédié. Le laboratoire de Génétique constitutionnelle de l'Institut Curie illustre bien cette problématique. Il a développé une activité de criblage des gènes de prédisposition aux cancers du sein et de l'ovaire (BRCAl et 2), du rétinoblastome $(R B 1)$ et de certaines maladies cassantes de l'ADN (ATM, FANCA, FANCG, NBS1, MRE11, RAD50). Ainsi, en 5 ans et sur les seuls gènes $B R C A I$ et $B R C A 2$, le nombre annuel de cas index analysés est passé de 168 à 466 cas et les tests des apparentés de 86 tests annuels à 429 . Au total, sur ces 5 dernières années, le nombre total d'amplicons analysés a bondi d'environ 23000 à $73000(+320 \%$ d'activité, hors activité de recherche) pour une équipe passée de 5 à 8 personnes sur la même période. Cette évolution a nécessité l'abandon de la gestion des données sous Excel pour migrer vers une base de données relationnelles à même de gérer l'analyse depuis l'arrivée des prélèvements au laboratoire jusqu'à l'impression du compte-rendu. La mise en place de cette base a débuté par la rédaction d'un cahier des charges qui exprimait les besoins principaux suivants : centralisation des données, gestion et suivi des différentes étapes des analyses, partage des informations au sein du laboratoire, évolutions possibles afin de s'adapter aux changements d'activité, interrogation des données via des requêtes complexes et import des données antérieures. Suite à l'appel d'offres, nous avons éliminé les progiciels présents sur le marché en raison de leur rigidité, coût et faible capacité d'évolution sans intervention extérieure. En revanche, nous avons retenu une solution flexible et conviviale sous FileMaker Pro ${ }^{\circ}$, proposée par un développeur indépendant «Carlo Baldassara, eConseil ». Les atouts majeurs de FileMaker Pro ${ }^{6}$ résident dans i) la possibilité de développer une base de données « à façon » ce qui permet une appropriation rapide par l'utilisateur ii) son évolutivité qui permet de s'adapter aux nouveaux gènes étudiés, aux nouvelles techniques d'analyses et ainsi gagner en réactivité. De la rédaction du cahier des charges jusqu'à la mise en production sous la forme d'une architecture client/serveur de la base il s'est écoulé moins d'un an. Notre base gère les données du prélèvement (date d'arrivée, lieu et forme du stockage, disponibilité), l'avancée des analyses, les étapes de validation technique et biologique et l'édition du compte rendu. D'autres fonctionnalités, comme les conditions d'analyses des gènes ont été importées. Les possibilités d'évolution ont permis d'implémenter de nouveaux modules d'analyses pour les nouveaux gènes étudiés. L'augmentation de notre activité n'aurait pu se faire sans cette base de données. Elle apporte un gain de temps au quotidien, une aide dans la gestion des analyses et une source d'information qui permet de répondre à différentes demandes (rapport d'activité, disponibilité de matériel, liste de mutation...). Nous pensons donc qu'une solution développée sous FileMaker Pro ${ }^{\circ}$ est à même de répondre aux besoins de nombreux laboratoires. Pour un bon fonctionnement, son implémentation et son développement doivent se faire en partenariat avec le service informatique, qui autorise les interactions de la base avec les autres bases de données de l'hôpital.

Mots-clés : base de données, FileMaker Pro, analyses.

DP452 / 569. DÉFICITS HYPOPHYSAIRE SYNDROMIQUES SANS RETARD MENTAL ET MUTATIONS DU GENE SOX3

I. Giurgea (1, 3), K. Machinis (1), M.-P. Vié-Luton (1), G. Pinto (4), B. Mignot (5), A.-M. Bertrand (5), C. Naud-Saureau (6), S. Rose (1), F. Kurtz (7), M. Legendre (1, 2), M.-L. Sobrier (1), J. Léger (8), P. Czernichow (8), S. Amselem $(1,2)$

(1) INSERM U654, Hôpital Armand-Trousseau, Paris, France; (2) AP-HP, Hôpital Armand-Trousseau, Service de Génétique et d'Embryologie médicales, Paris, France : (3) AP-HP, Groupe Henri Mondor-Albert Chenevier. Service de Biochimie et Génétique, Créteil, France; (4) AP-HP, Hopital Necker-Enfants-Malades, Service d'Endocrinologie, Paris, France; (5) Hôpital Saint Jacques, Service d'Endocrinologie, Besançon, France ; (6) Hôpital de Lorient, Service de Pédiatrie, Lorient, France; (7) Hôpital de Saint-Avold, Service de Pédiatrie, Saint-Avold, France; (8) AP-HP, Hôpital Robert-Debré, Service d'Endocrinologie, Paris, France

Introduction: Le phénotype de la souris dont le gène Sox3 a été invalidé a montré un défaut de la fonction hypophysaire, des anomalies de la mor- l'homme, des mutations du gène SOX3, localisé en Xq27.1, ont été identifiées chez de rares patients avec un retard de croissance en rapport avec un déficit en hormone de croissance (GH) et/ou un retard mental. Afin de mieux définir le spectre phénotypique de cette pathologie, nous avons étudié le gène SOX 3 chez des patients présentant un déficit hypophysaire syndromique. Patients et Méthodes Nous avons étudié 50 patients indépendants, de sexe masculin présentant un déficit en GH isolé ou combiné à d'autres déficits endocriniens de l'antéhypophyse et des anomalies de la ligne médiane. L'étude moléculaire du gène SOX3 a été réalisée par PCRséquençage direct. Résultats : Des mutations du gène SOX3 ont été identifiées chez 6 patients indépendants et chez deux de leurs frères présentant également un déficit antéhypophysaire. Des expansions de polyalanines $(+8$ et +11 Ala) ont été identifiées chez deux patients et chez chacun de leur frère atteint, tous les quatre ayant un déficit isolé en hormone de croissance et trois d'entre eux un strabisme. Une contraction de polyalanine ( -9 Ala) a été identifiée chez un patient présentant un déficit combiné en hormone de croissance, corticotrope, thyréotrope et gonadotrope associé à une anomalie du corps calleux. Des mutations faux-sens ont été observées chez trois patients : p.Ala102Ser chez un patient présentant une dysplasie septo-optique et des anomalies sévères de la ligne médiane; p.Ala5Pro chez un patient avec déficit isolé en hormone de croissance et troubles du comportement ; et p.Pro3Ser chez un patient avec déficit combiné en hormone de croissance, corticotrope et thyréotrope. Tous les patients avaient des anomalies morphologiques de l'hypophyse : antéhypophyse hypoplasique $(7 / 8)$, anomalies de la tige pituitaire $(5 / 7)$, et posthypophyse ectopique (4/8). En revanche, aucun d'entre eux ne présentait de retard mental. Conclusion : Nous rapportons 6 mutations (dont quatre nouvelles) du gène SOX3 chez des patients présentant un déficit hypophysaire syndromique dont les caractéristiques élargissent le spectre phénotypique de la pathologie nouvellement décrite associée aux mutations du gène SOX3.

Mots-clés : déficit hypophysaire, $S O X 3$, anomalies de la ligne médiane.

\section{- 453 / 578. ANALYSE FONCTIONNELLE DE VARIANTS DU GÊNE SPINKI IMPLIQUÉS DANS LES PANCRÉATITES HÉRÉDITAIRES}

A. Boulling $(1,2)$, C. Le Maréchal $(1,2,3,4)$, P. Trouvé $(1,2)$, O. Raguénès $(1,4)$, J.M. Chen $(1,3)$, C. Férec $(1,2,3,4)$

(1) Inserm U613, Brest; (2) Université de Bretagne Occidentale, Brest ; (3) Établissement Français du sang, Brest ; (4) Centre Hospitalier Universitaire, Brest

Introduction : Depuis maintenant plusieurs années, il est démontré que les variations observées sur le gène SPINKI codant le peptide PSTI (Pancreatic Secretory Trypsin Inhibitor) sont associées avec les pancréatites chroniques héréditaires. Ces altérations sont connues pour entraîner une baisse de l'activité ou de l'expression de PSTI. Nous avons récemment déterminé les conséquences fonctionnelles de trois variants situés sur le peptide signal (p.L12F, p.L14P, p.L14R), ainsi que celles de sept variants situés sur le peptide mature de PSTI (p.N34S, p.G48E, p.D50E, p.Y54H, p.P55S, p.R65Q, p.R67C). Matériels et méthodes: Les effets des différents variants ont été caractérisés par des western blot réalisés sur le type sauvage et les variants de PSTI, exprimés dans les cellules CHO. L'expression de ces variants a également été évaluée en mesurant leur niveau de transcription par RT-PCR. Résultats : Les altérations du peptide signal p.L14P et p.L14R entraînent une dégradation intracellulaire rapide de PSTI, empêchant ainsi sa sécrétion, tandis que p.L12F ne présente pas d'effet délétère. Les effets provoqués par les différents variants situés sur le peptide mature permettent de les classer en trois catégories. La première catégorie comprend les polymorphismes p.N34S et p.P55S, qui impliquent une conservation des propriétés physico-chimiques des acides aminés, et n'entrainnent pas de réduction de l'expression de PSTI. La deuxième catégorie contient uniquement la mutation faux-sens p.R65Q, substituant un acide aminé chargé positivement par un acide aminé neutre, et causant une réduction d'environ $60 \%$ de l'expression de la protéine. La dernière catégorie regroupent les mutants p.G48E, p.D50E, p.Y54H et p.R67C, impliquant des changements de charge des acides aminés et causant une inhibition complète ou presque complète de la sécrétion de PSTI. Les quantités d'ARNm mesurées pour le type sauvage et les mutants sont similaires, ce qui exclut la possibilité que les variations d'expression observées résultent d'une réduction de la transcription ou d'une baisse de la stabilité de l'ARNm. Conclusion: À la lumière de ces observations, il semble donc que ces mutations faux-sens entraînent une baisse de sécrétion de PSTI en perturbant les mécanismes de transports cellulaires de ce peptide. Travail de thèse financé par la région île de France, dans le cadre du projet PICRI en partenariat avec l'Association des Pancréatites Chroniques Héréditaires.

Mots-clés : Pancréatites chroniques, SPINKI, Analyse fonctionnelle. 


\section{-P454 / 590. GRANDS RÉARRANGEMENTS ET GÈNE DE LA FIBRILLINE DE TYPE 1 (FBN1): IMPLICATION NON NÉGLI- GEABLE CHEZ LES SUJETS PRÉSENTANT UN SYNDROME DE MARFAN INCOMPLET}

P. Khau Van Kien (1), N. Pallares-Ruiz (1), A. Plancke (1), L. OlivierFaivre (2), P. Sarda (3), C. Coubes (3), M. Claustres (1)

(1) Laboratoire de Génétique Moléculaire, CHU de Montpellier/INSERM U827; (2) Centre de Génétique, CHU de Dijon ; (3) Service de Génétique Médicale, $\mathrm{CHU}$ de Montpellier

Introduction : La précocité du diagnostic de syndrome de Marfan (établi grâce aux critères de Gand), constitue un réel défi car la prévention du risque aortique est d'autant plus efficace que celle-ci est débutée précocément (gain global de survie de l'ordre de 25 à 30 ans chez les patients correctement diagnostiqués et pris en charge). Les tests génétiques permettent d'évaluer indépendamment de l'âge un des critères majeurs de la classification de Gand. Malgré cet intérêt indéniable, le diagnostic moléculaire du syndrome de Marfan est un réel challenge et manque de sensibilité (rapportée selon les équipes entre 64 et $93 \%$ chez les sujets critères de Gand réunis et entre 42 et $60 \%$ chez les sujets - 1 critère). Débutée en Janvier 2005, la mise en place dans notre laboratoire d'une stratégie reposant sur le séquençage systématique et semi robotisé de la totalité des exons et bordures introniques des gènes $F B N 1, T G F B R 1, T G F B R 2$ a permit dans ses 2 premières années d'apporter une réponse positive à $86 \%$ des patients réunissant les critères de Gand $(n=57)$ et $61 \%$ des patients -1 critère de Gand $(n=62)$. Nous rapportons ici les résultats de la recherche complémentaire de grandes délétions et/ou duplications du gène FBN1, chez les sujets jusqu'alors négatifs. Matériels et méthodes : L'étude a porté sur 9 patients réunissant les critères de Gand et 24 patients - 1 critère, exhaustivement étudiés par séquençage double brin. La technique MLPA (Multiplex Ligation-dependent probe amplification) permettant d'étudier les 7 exons du gène TGFBR2 et 53 des 65 exons du gène $F B N 1$ a été utilisée en dépistage. Une validation a été systématiquement réalisée par PCR multiplex semi quantitative en utilisant 1) des amorces fluorescentes avec migration sur séquenceur $\mathrm{ABI} 3130 \mathrm{xl}$ et analyse des fragments à l'aide du logiciel GeneMapper 2) des amorces non fluorescentes et analyse par dHPLC (module fluorescent) à l'aide du logiciel Navigator. Résultats : 5 patients sur les 33 patients étudiés (1/9 patient réunissant les critères de Gand et $4 / 24$ patients Critères -1 ), présentent un grand réarrangement du gène $F B N 1$ : trois délétions et deux duplications ont été identifiées. Aucun réarrangement dans le gène TGFBR2 n'a été détecté. La sensibilité (taux de détection) du diagnostic moléculaire est désormais dans notre série de $88 \%$ chez les sujets réunissant les critères de Gand et de $68 \%$ chez les patients Critères -1 . Discussion: Dans notre série les grandes délétions et/ou duplications représentent $4,2 \%$ des anomalies du gène $F B N I$. À ce jour et à notre connaissance, aucune grande duplication n'est rapportée dans la littérature internationale et moins de 10 grandes délétions sur plus de 800 mutations sont décrites. La recherche de délétion/duplication permet d'augmenter le taux de détection de $2 \%$ pour les patients réunissant les critères de Gand et de $7 \%$ pour les patients Critères -1. Ces constatations, confrontées aux corrélations génotype/phénotype récemment mises en évidence dans le syndrome de Marfan, suggèrent que les grands réarrangements du gène $F B N I$, plus volontiers prédictives d'une haploinsuffisance que d'un effet dominant négatif, pourraient être plus fréquents chez les sujets présentant un syndrome de Marfan incomplet. Nous rappelons que pour ce groupe de sujets, l'identification d'un critère majeur supplémentaire selon la classification de Gand (notamment du critère génétique) peut permettre la mise en place d'une prévention efficace vis-à-vis de complications potentiellement mortelles.

Mots-clés : Marfan, FBNI, grands réarrangements.

\section{IP455 / 595. NOUVELLES MUTATIONS DE ZMPSTE24 (FACE1) IMPLIQUÉES DANS LA DERMOPATHIE RESTRICTIVE ET POINT SUR LA LITTÉRATURE}

C. Navarro (1), V. Esteves-Vieira (2), A. Boyer (2), S. Courrier (1), C. Beroud (3), C. Badens (1, 2), M. Wehnert (4), A. De Sandre-Giovannoli $(1,2)$, N. Lévy $(1,2)$

(1) UMR910, Faculté de Médecine de Marseille, France ; (2) Laboratoire de Génétique Moléculaire, CHU de la Timone, Marseille, France; (3) U827, IURC, Institut Universitaire de Recherche Clinique, Montpellier ; (4) Institute of Human Genetics, University of Greifswald, Germany

Introduction : La Dermopathie Restrictive (DR) est un syndrome progéroïde parmi les plus sévères, habituellement létale en période néonatale. Les principaux signes cliniques sont une peau épaisse et rigide, de multiples contractures articulaires, une diminution généralisée de la densité osseuse et une insuffisance pulmonaire causant le décès. Nous avons récemment identifié la DR, comme étant une maladie récessive liée à des mutations dans ZMPSTE24 (FACE1). Ce gène code pour une métalloprotéase spécifique de la maturation post-traductionnelle de la lamine A. Toutes les mutations trouvées dans ZMPSTE24 causant la DR sont des mutations nulles entraînant, par conséquent, l'accumulation de la lamine A sous la forme de son précurseur, appelé Prélamine A, à l'état farnésylé. Le même mécanisme physiopathologique, impliquant un précurseur tronqué de Prélamine A, est également en cause dans la Progéria de Hutchinson-Gilford (HGPS). Resultats : Nous rapportons ici 6 nouvelles mutations récessives, identifiées chez 5 patients atteints de DR classique, incluant une mutation faux sens, 2 délétions et 3 mutations affectant directement les sites consensus d'épissage. Nous avons également identifié 6 nouvelles familles avec un ou deux enfants atteints, portant la mutation récurrente c.1085_1086dupT, localisée dans l'exon 9 du gène. Egalement, nous avons mis en évidence plusieurs nouvelles variations de séquence associés ou non à une pathologie, au cours d'une large étude de patients atteints de syndromes progéroïdes. Toutes les mutations et variations de séquences inclues dans nos études et toutes celles qui ont été publiées par ailleurs ont été regroupées dans une base de données UMD-ZMPSTE24, bientôt accessible on-line à toute la communauté scientifique. Pour chaque mutation, les caractéristiques moléculaires, protéiques et les informations cliniques sont spécifiées. Cette base de données constituera un outil de travail précieux, aidant les future recherches effectuées autour de ce gène et des pathologies associées. Conclusion: Les mutations rapportées ici, dans 11 nouvelles familles présentant un ou plusieurs enfants atteints de $\mathrm{DR}$, démontrent clairement que le gène ZMSPTE24 est le gène à cibler en cas de diagnostic de DR, bien qu'il puisse être impliqué par ailleurs, dans d'autres syndromes progéroîdes moins sévères.

Mots-clés : progeroide, ZMSPTE24, dermopathie.

\section{IP456 / 596. EFFET DES VARIATIONS DE PCSK9 SUR LES TAUX DE CHOLESTÉROL PLASMATIQUE}

M. Abifadel (1, 2), J. Bonneau (1), A. Marques (1), M. Marduel (1), M. Devillers (1), D. Erlich (1), C. Junien (1, 3), A. Munnich (1), J.-P. Rabès $(1,3)$, M. Varret $(1), C$. Boileau $(1,3)$

(1) INSERM U781, Hôpital Necker, Paris; (2) Faculté de Pharmacie, Université Saint-Joseph, Beyrouth, Liban; (3) Laboratoire de Biochimie, d'Hormonologie et de Génétique Moléculaire, AP-HP, Ambroise-Paré, Boulogne, France

L'hypercholestérolémie à transmission autosomique dominante (ADH) est l'une des maladies génétiques les plus fréquentes. Les gènes codant le récepteur des lipoprotéines de faible densité (LDLR) et son ligand l'apolipoprotéine B ont été longtemps les seuls incriminés dans l'ADH. Nos travaux ont permis d'identifier en 2003 , le $3^{e}$ gène impliqué dans cette maladie: PCSK9, (Proprotéine Convertase Subtilisine Kexine 9). Plusieurs mutations hypercholestérolémiantes de PCSK9 (p.S127R, p.F216L, p.D374Y...) entrainent un gain de fonction provoquant une diminution des taux de récepteurs des LDL. Tandis que d'autres variations sont hypocholestérolémiantes et associées à une diminution de l'incidence des accidents cardiovasculaires: p.Y142X, p.C679X (retrouvées chez $2 \%$ des afro-américains) et p.R46L (fréquence de $3,2 \%$ chez les blancs). Les variations de $P C S K 9$ sont des facteurs majeurs modifiant les taux de cholestérol sanguin, mais certaines présentent une répartition géographique assez hétérogène. Nous avons étudié la fréquence des variations de PCSK 9 chez 600 sujets caucasiens ( 400 hypercholestérolémiques et 200 normolipémiques). La variation p.R46L a été uniquement retrouvée dans la population normolipémique, à une fréquence de $2 \%$. Nous avions décrit la variation p.A443T pour la première fois chez une patiente hypercholestérolémique française de 50 ans. Cette variation n'était pas retrouvée chez 340 témoins caucasiens. Elle semble cependant être un polymorphisme rare chez les blancs, plus fréquent chez les noirs, associé à des taux bas de LDL-cholestérol mais retrouvé aussi bien chez des sujets hyper qu'hypocholestérolémiques dans l'étude de Dallas. Enfin, la variation p.R237W que nous avions initialement décrite chez une patiente canadienne hypercholestérolémique, ne semble retrouvée que chez des sujets hypercholestérolémiques dans l'étude de Dallas bien qu'elle ait été associée à une diminution des taux de LDL-cholestérol par Berge et al. La caractérisation des variations de PCSK9 est primordiale pour une meilleure compréhension du rôle de cette enzyme dont l'impact sur la cholestérolémie est majeur. Les inhibiteurs éventuels de PCSK9 ouvriraient la voie vers de nouvelles cibles thérapeutiques de l'hypercholestérolémie, potentialisant les effets des statines.

Mots-clés : hypercholestérolémie, PCSK9, maladies cardiovasculaires. 


\section{-1P457 / 600. MISE EN PLACE DU DIAGNOSTIC MOLÉCULAIRE} DE LA MALADIE DE FANCONI

C. Dubois d'Enghien (1), A. Laugé (1), D. Chamousset (2), J. Soulier (2), D. Stoppa-Lyonnet (1)

(1) Institut Curie Laboratoire de génétique constitutionnelle ; (2) Laboratoire d'Hématologie et Centre de Référence « Aplasies Médullaires Constitutionnelles », Hôpital St Louis APHP

La maladie de Fanconi est caractérisée par un syndrome malformatif congénital variable, une insuffisance médullaire d'apparition retardée et un risque très élevé de leucémie aiguë myéloïde et de carcinomes. Le diagnostic est rarement fait à un stade précoce et peut être difficile devant une insuffisance médullaire isolée. C'est une maladie génétique transmise selon le mode récessif, très hétérogène : 13 groupes de complémentation cellulaire et les gènes correspondants ont été identifiés. Cependant trois gènes rendent compte de $90 \%$ des cas en France : FANCA, FANCC et FANCG. Le diagnostic, suspecté devant une insuffisance médullaire ou un syndrome malformatif, conduit à l'indication d'un caryotype réalisé en présence d'agents pontants (mitomycine $\mathrm{C}$, diépoxy-butane ou mechlorethamine). L'analyse de l'ubiquitination de la protéine FANCD2 confirme le diagnostic, permet de classer les patients selon le niveau de l'atteinte de la voie FA/BRCA et oriente l'analyse moléculaire. Aujourd'hui, le diagnostic et la caractérisation de la maladie de Fanconi reposent sur les analyses cytogénétiques et fonctionnelles et sur l'identification du gène responsable par complémentation rétrovirale et/ou l'analyse directe des gènes les plus fréquemment impliqués (FANCA, FANCG). L'étude moléculaire est réalisée depuis 2006 après validation clinique et biologique de l'indication. Le gène FANCA est composé de 43 exons codants soit 1456 acides aminés, il a été étudié en séquençage direct pour les mutations ponctuelles et en MLPA $^{\circ}$ (Multiplex Ligation dependant Probe Amplification) pour les réarrangements de grande taille. Le gène FANCA a été étudié chez 88 patients à partir d'ADNs extraits de sang circulant et/ou de fibroblastes : 134 mutations inactivatrices ont été identifiées chez 73 patients. Parmi ces mutations, $47(35 \%)$ sont des délétions de 1 à 43 exons, $39(29 \%)$ sont des mutations non-sens, des insertions ou délétions de quelques nucléotides, $22(16 \%)$ touchent un site d'épissage, $17(13 \%)$ sont des délétions respectant le cadre de lecture et $9(7 \%)$ sont des mutations faux-sens rapportées comme délétères dans la base du Rockfeller center (http://www.rockefeller.edu//fanconi/mutate/). Parmi les 73 patients porteurs d'au moins une mutation FANCA, 62 présentent les 2 mutations attendues et 11 , une seule. Notons cependant que dans 10 de ces 11 cas, un variant de signification inconnue a également été identifié. Pour 5 patients, les 2 mutations FANCA n'étaient présentes qu'au niveau des fibroblastes, indiquant la survenue d'une réversion au niveau médullaire. Aucune mutation n'a été détectée chez 15 patients : 7 se sont révélés secondairement appartenir aux groupes C ou G, 6 n'ont pas été documentés par complémentation, et 2 appartenant au groupe A présentent des variants de signification inconnue. Des études complémentaires sont en cours sur ces variants. Chez 6 des 8 derniers patients, l'analyse a été faite à partir de fibroblastes éliminant ainsi une non détection de mutation au niveau lymphocytaire secondaire à une réversion médullaire. Le gène FANCG comprend 14 exons codants soit 623 acides aminés étudiés aujourd'hui en séquençage direct uniquement. Cinq patients ont été étudiés sur le gène $F A N C G: 2$ mutations inactivatrices (introduisant un codon stop) ont été détectées chez chaque patient. Le taux de mutations identifiées dans les gènes FANCA et FANCG est élevé. L'approche moléculaire devrait faciliter le diagnostic prénatal et le diagnostic pré-implantatoire pour les parents ayant un enfant atteint. Elle permet aussi, lorsque le diagnostic est incertain, de confirmer le diagnostic de maladie de Fanconi et par là de mieux prendre en charge l'enfant. Médecins ayant collaboré à l'étude : E Gluckman, T Leblanc, A Baruchel, L Degos, F Bernaudin, Y Bertrand, O Boespflug-Tanguy, H Esperou, C Francannet, O Hermine, V Mialou, B Nelken, C Pondarré, P Rohrlich, G Socié, C Thauvin, P Turlure.

Mots-clés : FANCA, FANCG, mutations.

QP458 / 605. TRANSMISSION RÉCESSIVE D'UN DÉFICIT ISOLÉ EN HORMONE DE CROISSANCE ASSOCIÉ À DEUX NOU. VELLES MUTATIONS DU RÉCEPTEUR DE LA GHRÉLINE (GHSR)

M. Legendre (1), J. Pantel (1), S. Nivot-Adamiak (2), S. Morisset (3), M.-P. Vie-Luton (1), Y. le Bouc (4), J. Epelbaum (5), S. Amselem (1) (1) INSERM U654, Hôpital Trousseau, Paris, France ; (2) Service d'Endocrinologie pédiatrique, CHR de Rennes, Paris, France ; (3) INSERM U573, Centre Paul-Broca, Paris, France; (4) Service d'Explorations fonctionnelles endocriniennes, Hôpital Trousseau, Paris, France; (5) INSERM
Introduction : La ghréline est une hormone pléiotrope initialement décrite pour ses propriétés sécrétrices d'hormone de croissance. La ghréline constitue également la première hormone orexigène décrite à ce jour. Elle exerce ces propriétés via l'isoforme la du Growth Hormone Secretagogue Receptor (GHSR). Le GHSR est un récepteur à sept segments transmembranaires couplé aux protéines $\mathrm{G}$ qui présente une forte activité constitutive. Nous avons récemment impliqué le GHSR en pathologie humaine chez des patients présentant une petite taille familiale. Au sein des deux familles identifiées, la mutation p.Ala204Glu ségrège avec la petite taille selon un mode dominant. Nous décrivons ici l'implication du gène GHSR dans un cas de déficit isolé en hormone de croissance de transmission autosomique récessive, à la lumière de l'analyse fonctionnelle des variants impliqués. Patients : Le cas index présente une petite taille $(-3,0$ D.S.) associée à un déficit isolé en hormone de croissance, ainsi qu'un Indice de Masse Corporelle au 3 e percentile. L'analyse génétique du GHSR a révélé que ce patient est hétérozygote composite [p.Trp2X ; p.Arg237Trp]. Ses parents, respectivement hétérozygotes pour la mutation non-sens précoce p.Trp $2 X$ et la mutation faux-sens p.Arg237Trp, présentent une taille et un poids compris dans les valeurs normales. Méthodes : L'analyse fonctionnelle in vitro a été effectuée dans des lignées cellulaires exprimant transitoirement le GHSR ou ses variants dotés d'un tag HA. L'affinité des récepteurs a été évaluée par la mesure de la liaison de la ghréline radiomarquée (membranes de cellules COS-7). La capacité des récepteurs à répondre à la ghréline par la voie de la phospholipase $\mathrm{C}$ a été évaluée grâce à un rapporteur calcique fluorescent (cellules HEK 293). Leur activité transcriptionnelle a été mesurée par des expériences de co-transfection avec un plasmide contenant un rapporteur Luciférase en aval de sites Serum Response Element (cellules HEK 293). L'expression des récepteurs à la surface cellulaire a été mesurée par des tests ELISA (cellules HEK 293). Résultats - Le variant p.Trp $2 X$ du GHSR présente une activité constitutive et une réponse à la ghréline effondrées. Le variant p.Arg237Trp montre une perte partielle de son activité constitutive alors que sa réponse à la ghréline est conservée. Cette altération partielle de l'activité constitutive du récepteur est un phénomène intrinsèque puisque l'expression à la surface de la cellule du variant p.Arg237Trp est comparable à celle du GHSR sauvage. Conclusion - Nous rapportons le premier cas de déficit en hormone de croissance associé au GHSR de transmission autosomique récessive. L'analyse fonctionnelle des variants confirme l'importance du GHSR dans la croissance somatique chez l'homme et souligne le rôle crucial de l'activité constitutive de ce récepteur.

Mots-clés: Croissance, Récepteur ghréline, Déficit en hormone de croissance.

\section{D459 / 619. ISODISOMIE UNIPARENTALE PARTIELLE DU} CHROMOSOME 17P DANS UN CAS DE CYSTINOSE INFANTILE A.S. Lebre (1), V. Morinière (1), O. Dunand (3), N. Morichon (1), C. Antignac $(1,2)$

(1) Département de Génétique, Hôpital Necker-Enfants Malades, Paris, France; (2) Inserm U574, Hôpital Necker-Enfants Malades, Paris, France; (3) Hôpital Trousseau, Paris, France

La cystinose est une maladie autosomique récessive caractérisée par une accumulation de cystine intra-lysosomale due à un défaut du transport de la cystine à travers la membrane lysosomale. Trois formes cliniques de cystinose (infantile, juvénile et forme oculaire de cystinose) ont été identifiées en se basant sur la sévérité des symptômes et l'âge de début de la maladie. Le gène en cause est le gène CTNS situé sur le chromosome 17 en $17 \mathrm{p} 13$. Ce gène couvre $23 \mathrm{~kb}$ de séquence génomique et code pour le transporteur lysosomal de la cystine, ou cystinosine. Des mutations du gène CTNS ont été détectées dans une série de patients présentant toutes les formes de cystinose. La mutation la plus fréquente est une grande délétion de $57 \mathrm{~kb}$ emportant les exons 1 à 10 du gène CTNS. Cette délétion a été détectée chez 60 à $70 \%$ des patients d'Europe du nord. Nous décrivons ici une isodisomie uniparentale partielle d'origine maternelle du chromosome 17 (mat UPD17) chez une fillette de 30 mois présentant une forme isolée de cystinose infantile. Une délétion homozygote de $57 \mathrm{~kb}$ a été identifiée dans le gène CTNS. La mère de cette fillette est hétérozygote pour la délétion mais de façon étonnante, son père n'est pas porteur de la délétion. L'étude des marqueurs microsatellites couvrant le chromosome 17 démontre l'absence de contribution paternelle pour tous les marqueurs du chromosome 17 . Une seule contribution maternelle a été identifiée pour le bras court du chromosome 17. Une délétion 17p n'étant pas viable, ces résultats suggèrent la co-existence d'une disomie uniparentale d'origine maternelle avec hétérodisomie $17 \mathrm{q}$ et isodisomie $17 \mathrm{p}$. Il s'agit du premier cas d'isodisomie uniparentale partielle du chromosome $17 \mathrm{p}$.

Mots-clés : isodisomie uniparentale UDP17, cystinose, gène CTNS. 
IP460 / 623. SÉQUENCAGE DE L'ADN MITOCHONDRIAL À L'AIDE DU KIT DE RESÉQUENCAGE MITOSEQR ${ }^{\circ}$ DANS LE CADRE DU DIAGNOSTIC MOLECULAIRE DES MALADIES MITOCHONDRIALES

C. Jardel (1, 3), F. Delbos (1), S. Filaut (1), I. Lemiere (1), P. Laforêt (2, 3), A. Lombès (3), B. Hainque $(1,3)$

(1) Service de Biochimie, UF de cardio et myogénétique; (2) Centre de référence des maladies neuromusculaires; (3) INSERM U5823, Groupe hospitalier Pitié-Salpêtrière-APHP-75013-Paris

Les maladies mitochondriales constituent un ensemble très hétérogène de pathologies dâ à un dysfonctionnement de la chaîne respiratoire. À ce jour, moins de $20 \%$ des patients atteints bénéficient du diagnostic moléculaire; ceci tient à 2 raisons : 1) les gènes nucléaires responsables de ces maladies sont potentiellement très nombreux $(>1000) 2$ ) l'analyse exhaustive de l'ADN mitochondrial ( $16,5 \mathrm{~Kb}$ ) est rarement réalisée. Pour réaliser celle-ci, la technique utilisée doit permettre de détecter à la fois les mutations homoplasmiques et hétéroplasmiques quelqu'en soit le taux. Le but de ce travai a été de tester le kit de resequençage MitoSEQr ${ }^{6}$ Applera, pour apprécier son intérêt en pratique diagnostique. Matériel et méthodes : le kit MitoSEQr ${ }^{* 0}$ consiste en un set de 46 paires de primers choisis pour permettre le séquençage de tout l'ADN mitochondrial de façon optimisée (conditions uniques de PCR et l'utilisation de queues M13). Le séquençage a été réalisé sur AbiPrism 3700, selon les recommandations du fabricant et les séquences analysées à l'aide du logiciel SeqScape et du projet MitoSEQr ${ }^{\circ}$ Le génome mitochondrial entier a été analysé pour 8 patients atteints d'un déficit de la chaîne respiratoire. La détection de l'hétéroplasmie a été évaluée sur huit échantillons de patients porteurs de mutations m.3243A>G (mutation MELAS) et m.8344A $>\mathrm{G}$ (mutation MERRF) à des taux d'hétéroplasmie variables. Résultats : les difficultés de purification des produits de PCR dus aux petits volumes préconisés nous ont amenés à modifier notre protocole et à employer l'exo-sap ${ }^{\circ}$. L'utilisation du kit s'est avérée par la suite satisfaisante et a permis l'obtention de réactions de séquence de qualité dans plus de $95 \%$ des cas. La mutation m.3243A $>\mathrm{G}$ a été détectée pour les taux d'hétéroplasmie de $20 \%, 50 \%$ et $60 \%$; par contre la mutation n'est pas détectée pour un taux inférieur à $10 \%$. La mutation $\mathrm{m} .8344 \mathrm{~A}>\mathrm{G}$ a été détectée pour les taux d'hétéroplasmie de $25 \%$ et $75 \%$. Ces résultats techniques sont satisfaisants même si le seuil de détection reste inférieur à celui de la DGGE. L'estimation théorique du coût et du délai d'obtention des résultats du séquençage de tout l'ADN mitochondrial est respectivement de $750 €$ et 72 heures. Par contre, au niveau du proje MitoSEQr ${ }^{B}$, nous avons mis en évidence un certain nombre d'erreurs dans la séquence de référence, dans le sens de lecture de certains gènes et dans la numérotation des amorces ; celles ci ont en partie été corrigées mais des améliorations sont souhaitables pour une utilisation plus agréable et plus facile en pratique diagnostique.

Mots-clés : ADN mitochondrial, reséquençage, diagnostic.

\section{P461 / 625. ALAMUT, ASSISTANT INFORMATIQUE DU GÉNÉ-} TICIEN MOLÉCULAIRE

A. Blavier, S. Guennou, F. Wolinski

Interactive Biosoftware

La validation médicale par le généticien moléculaire d'une analyse génétique, basée sur la séquence, nécessite l'interprétation de la variation détectée. Le problème de l'interprétation de la variation est particulièrement important pour les mutations faux-sens et nécessite de disposer des informations relatives à la fréquence allélique des variants, à la conservation phylogénétique des acides aminés touchés et l'impact sur la structure de la protéine. De très nombreuses données moléculaires, potentiellement utiles à l'interprétation de tels variants et au diagnostic moléculaire des maladies géniques, sont disponibles sur les serveurs publics. Néanmoins, leur dissémination, leurs modalités d'accès et de représentation sont très variables et ne permettent pas de les utiliser en pratique régulière. L'objectif a donc été de créer un système logiciel jouant le rôle de véritable assistant informatique du généticien moléculaire, après la réalisation de l'analyse génétique. Si la vocation essentielle de ce système est l'interprétation de mutations, il a été conçu avec deux objectifs supplémentaires, d'une part l'adaptation progressive de méthodes de calcul prédictive et d'autre part la prise en compte des règles de nomenclature de l'HGVS afin de faciliter l'édition d'un compte-rendu optimisé de génétique moléculaire. En étroite coopération avec le laboratoire de génétique moléculaire du CHU de Rouen, nous avons donc réalisé un système logiciel d'intégration et de visualisation des données moléculaires facilitant l'interprétation des mutations. Son principe central est de rassembler, dans un outil cohérent et productif, un ensemble d'informations facilement exploitables dans la démarche diagnostique. Ces informations s'appuient sur les grandes bases des données génomiques et post-génomiques publiques. Le système est constitué de 2 composants principaux : un logiciel graphique interactif de représentation et de manipulation des données et un serveur de données intégratif. Ces 2 composants communiquent entre eux par le protocole standard http, ce qui permet un déploiement simple du logiciel graphique dans les laboratoires de diagnostic. Ce logiciel est un «navigateur de gènes » incorporant des capacités de prédiction sur les conséquences calculables des mutations, et intègre un ensemble de fonctionnalités génériques pour la manipulation des séquences et une connaissance détaillée de la nomenclature des mutations. Le logiciel Alamut a été élaboré à contrepied du « tout web " qu'offrent la plupart des outils de présentation des données dans le domaine, tels que les navigateurs de génomes. Si Alamut utilise effectivement les technologies du web, son architecture et son interactivité lui permettent de dépasser les limites des browser généralistes qui n'ont pas la capacité de manipuler efficacement les données spécialisées. Ce logiciel, commercialisé depuis juin 2007, a déjà été diffusé dans une dizaine de laboratoires de diagnostic moléculaire en Europe. Le positionnement de ce logiciel dans le processus diagnostique en aval de l'analyse de séquences permet de systématiser et de faciliter une phase du diagnostic jusqu'alors très peu assistée. Ce logiciel devrait faciliter et améliorer la qualité du diagnostic moléculaire des maladies géniques.

Mots-clés : diagnostic, faux-sens, logiciel.

1962 / 630. MUTATIONS DU GENE MFN2 CHEZ DES PATIENTS ATTEINTS DE FORMES AUTOSOMIQUES RÉCESSIVES DE MALADIE DE CHARCOT-MARIE-TOOTH AXONALE

C. Magdelaine (1), G. Nicholson (2), P. De Mas (3), D. Zhu (2), M. Ryan (4), F. Sturtz (1), J.-M. Vallat (5), R. Ouvrier (6), B. Funalot (5)

(1) Service de biochimie et génétique moléculaire, CHU de Limoges; (2) Molecular Medicine Laboratory \& ANZAC Research Institute, Concord Hospital, Sidney NSW, Australie ; (3) service de génétique médicale, CHU de Toulouse; (4) Neurosciences Department, Royal Children's Hospital, Murdoch Children's Research Institute, Victoria, Australie ; (5) Service et laboratoire de neurologie, CHU de Limoges; (6) Institute for Neuromuscular Research, The Children's Hospital at Westmead, Westmead, Australie

Introduction: Les neuropathies sensitivo-motrices axonales à début précoce constituent un groupe hétérogène de neuropathies périphériques. Chez certains patients, des mutations hétérozygotes du gène MFN2 ont été identifiées. Il s'agit la plupart du temps de cas sporadiques porteurs de mutations dominantes de novo, classés comme des formes sévères à début précoce de maladie de Charcot-Marie-Tooth de type 2, sous-type A2 (CMT2A2). L'analyse moléculaire du gène MFN2 chez quatre patients atteints d'une telle forme axonale à début précoce a en fait montré la présence non pas d'une seule mutation à l'état hétérozygote mais celle de deux mutations hétérozygotes composites ( 3 cas) ou de la même mutation à l'état homozygote $(1$ cas $)$. Les patients appartenaient à quatre familles (trois familles australiennes et une famille française), avec dans trois cas un seul enfant atteint et dans un cas trois enfants atteints. Objectif : Le but de la présente étude est d'étudier le mode de transmission de la maladie dans ces quatre familles comportant six cas de neuropathie axonale sévère à début précoce et porteurs de mutations homozygotes ou hétérozygotes composites du gène $M F N 2$. Methodes: Les données cliniques, paracliniques et moléculaires des six patients et de leurs huit parents ont été étudiées. Resultats : Les six patients sont porteurs d'une mutation sur chacun des allèles du gène $M F N 2$. L'âge de début de la maladie est compris entre 2 et 3 ans chez tous les cas index. Deux d'entre eux présentent une surdité modérée. Chacun des huit parents est porteur hétérozygote d'une des mutations présentes chez son (ses) enfant(s) atteint(s). Tous les parents sont asymptomatiques à l'interrogatoire mais l'examen clinique approfondi met en évidence chez certains d'entre eux des signes cliniques en faveur d'une dysfonction modérée des nerfs périphériques (pieds creux, déficit moteur et/ou sensitif distal léger). Trois d'entre eux ont des problèmes d'audition. Conclusion: Des mutations homozygotes ou hétérozygotes composites du gène $M F N 2$ peuvent être responsables de formes autosomiques récessives de maladie de Charcot-Marie-Tooth axonale (ARCMT2) à début précoce. Un caractère semi-dominant de certaines des mutations peut cependant être discuté du fait de l'existence de signes cliniques légers chez plusieurs des parents.

Mots-clés : Charcot-Marie-Tooth, mitofusine 2, MFN2.

IP463 / 639. LE CRIBLAGE DU GENE APC PAR LA TECHNIQUE CSGE DANS LA POPULATION IRANIENS ATTEINTS DE POLY. POSE ADÉNOMATEUSE FAMILIALE

N. Vasli (1), M. Noruzinia (2)*, M. Azmi (3), M. Omrani (3), A. Saremi (4) 
(1) Department of genetics, School of Sciences, Azad Olum Tahghighat University, Tehran, Iran; (2) Department of Medical Genetics, School of Medical Sciences, Tarbiat Modarres University, Tehran, Iran ; (3) Institute Cancer of Tehran University ; (4) Sarem Community Hospital and Medical Center ; *Corresponding author : mehrnoruzinia@yahoo.fr

Introduction : La Polypose recto-colique familiale est une maladie héréditaire à transmission autosomique dominante. Des centaines voire des milliers de polypes coliques apparaissent vers l'âge de 16 ans en moyenne (fourchette de valeurs 7-36 ans) qui en l'absence de colectomie (ablation du colon) dégénèrent systématiquement en cancer qui apparaissent vers l'âge de 39 ans (fourchette de valeurs 34-43 ans). Le gène responsable est $A P C$. Le gène $A P C$ possède 15 exons et code pour une protéine de 2843 acides aminés. Une mutation germinale a été identifiée dans plus des $2 / 3$ des malades atteints, inactivant les fonctions de transcription du gène. La plupart des mutations du gène APC sont situées en MCR (Mutation Cluster Region). Nous avons réalisé une techniques simple de criblage de mutation sur ce gène. Nous avons étudié la prévalence de mutations de ce gène dans une population de malades atteints de forme classique de FAP. Matériaux et Méthode : nous avons fait la mise au point de technique CSGE pour le gene $A P C$. L'ensemble de la région codante du gène $A P C$ a été amplifié dans 26 réactions de PCR. Après « l'hétéroduplexing » les fragments ont été migrés sur un gel dénaturant de « l'acryl-BAP ». la technique originale a été modifiée pour avoir une meilleure résolution et un moindre temps de migration. Cette technique a été appliquée sur 10 patients iraniens atteints de FAP et 5 patients contrôles positifs. Le séquençage direct a été utilisé pour vérifier les résultats de CSGE. Résultats : nous avons pu identifier la mutation délétère dans 8 patients et toutes les 5 mutations délétères dans les contrôles positifs. Seulement $20 \%$ des mutations sont situées sur la région MCR Conclusion : Conformation que la Sensitive Gel Electrophoresis est une technique accessible pour le criblage du gène $A P C$. Dans les pays où le séquençage direct n'est accessible à tous les laboratoires un criblage de mutations basé sur la technique de CSGE semble accessible. Notre résultat montre une différence dans la répartition des mutations sur le gène $A P C$.

Mots-clés : APC, FAP, CSGE.

\section{IP464 / 647. SYNDROME D'OMENN : PREMIÈRE OBSERVA- TION MAROCAINE AVEC UNE MUTATION DELT631 DU GENE RAGI}

I. Cherkaoui Jaouad (1), K. Ouldim (1), S. Chafaï Elalaoui (1), Y. Krioule (2), A. Villa (3), A. Sefiani (1)

(1) Département de Génétique Médicale, Institut National d'Hygiène, Rabat, Maroc, Laboratoire International Associé INSERM ; (2) Service de pédiatrie II, CHU Ibn Sina, Rabat, Maroc ; (3) CNR-ITB, Institut de Technologie et de Biomédecine, Milan, Italie

Le syndrome d'Omenn est un déficit immunitaire combiné sévère qui apparaît chez l'enfant dès les premiers mois de la vie, caractérisé par une hyperéosinophilie massive avec hyperleucocytose. Il se manifeste par une éruption de vésicules rouges sur la peau et les muqueuses, une tuméfaction des ganglions lymphatiques, de la rate et du foie, associée à des infections de la peau et des poumons. Cette maladie rare, de transmission autosomique récessive, est liée à des mutations hypomorphes des gènes de recombinaison RAG1 et RAG2 (Recombinating activating Genes) et du gène ARTEMIS et plus récemment, des mutations des gènes IL7RA et RMRP ont été incriminées dans ce déficit immunitaire. Nous rapportons la première observation marocaine, d'un nourrisson âgé de quatre mois issu d'un mariage consanguin, présentant des signes en faveur du syndrome d'Omenn. L'étude moléculaire nous a permis de confirmer ce diagnostic en mettant en évidence une délétion delT631 (c.631delT) du gène RAG1. Cette mutation a été déjà rapportée chez un enfant d'origine algérienne ; ce qui est en faveur d'un effet fondateur au Maghreb.

Mots-clés : syndrome d'Omenn, gène $R A G$, effet fondateur.

EP465 / 648. ENHANCED MISMATCH MUTATION ANALYSIS (EMMA), UNE NOUVELLE MÉTHODE DE DÉTECTION SIMULTANÉE ET À HAUT DÉBIT DE MUTATIONS PONCTUELLES ET DE RÉARRANGEMENTS DE GRANDE TAILLE

J. Weber (1), J. Champ (2, 3), S. Berhouet (2), V. Moncoutier (2), D. Michaux (2), B. Gastaldello (4), F. Bonnet (4), N. Sévenet (4), C. Houdayer (2), D. Stoppa-Lyonnet (2), J.-L. Viovy (3)

(1) Fluigent, Paris ; (2) Service de génétique oncologique, Institut Curie, Paris ; (3) Laboratoire de Physico-Chimie-Institut Curie, Paris ; (4) Laboratoire de génétique moléculaire, Institut Bergonié, Bordeaux

Introduction et rationnel : Le criblage de mutations géniques peut s'avérer nombreux variants polymorphes. Le séquençage direct considéré comme méthode de référence reste coûteux et ne présente pas une sensibilité absolue. Ainsi, de nombreux laboratoires préferent utiliser une stratégie de pré criblage des variants suivi de leur séquençage. Parmi les techniques de criblage, la dHPLC était, jusqu'à récemment, la technique semblant offrir le meilleur compromis entre sensibilité, robustesse et coût malgré son faible débit et la nécessité d'une expertise technique. Ainsi, la mise en cuvre de méthodes rapides, fiables, combinant la détection de mutations de petite et de grande taille et nécessitant peu de mise au point permettrait une économie en temps et en coût pour les laboratoires de diagnostic moléculaire, qui font face à une augmentation de la demande de tests génétiques. Nous proposons ici une méthode, EMMA (Enhanced Mismatch Mutation Analysis), permettant de détecter simultanément les mutations ponctuelles et les réarrangements de grande taille (RGT) avec un débit et une productivité très supérieurs à la dHPLC. Matériel et méthodes: La technologie EMMA est basée sur l'analyse d'hétéroduplexes en électrophorèse capillaire. Le développement d'une matrice de séparation spécifique comprenant des additifs permettant d'accroître la résolution a permis d'obtenir une sensibilité compatible avec une utilisation en diagnostic. Une condition unique de séparation permet d'étudier tous les types de fragments de PCR de taille comprise entre 200 et $550 \mathrm{pb}$. L'utilisation du multiplexage en taille, dès l'étape de PCR permet d'analyser environ 1500 fragments/jour sur un séquenceur comprenant 16 capillaires et de diminuer considérablement le nombre de PCR. Un logiciel dédié permet une analyse rapide et semi-automatisée des résultats. Enfin, EMMA est compatible avec une analyse semi-quantitative permettant de détecter les RGT simultanément aux mutations ponctuelles. Les résultats sont présentés pour le gène $B R C A 1$, dont le criblage par EMMA repose sur l'analyse de 31 fragments répartis en 9 multiplex (au lieu de 35 PCR pour le criblage par dHPLC), avec pour chacun un fragment de référence essentiel à la détection des RGT. Résultats et Discussion: Une validation en aveugle, comparativement à la dHPLC (et à la QMPSF pour les RGT), a été réalisée sur l'ADN constitutionnel de 279 patientes de l'Institut Curie, générant 2511 réactions d'amplification en PCR multiplex (8649 fragments). Cette analyse a été réalisée par une personne en 6 semaines, soit une économie de temps d'analyse d'un facteur supérieur à 3. La totalité des mutations (ponctuelles et RGT) a été détectée. L'analyse des 8649 fragments a entrainé moins de $5 \%$ de vérifications en EMMA, $1,3 \%$ en séquençage et un taux de faux-positifs inférieur à $0,04 \%$. Par ailleurs la technique a été implantée avec succès dans un autre laboratoire, le laboratoire de génétique moléculaire (Institut Bergonié) permettant le criblage de la base des témoins positifs BRCAl ( 65 mutations ponctuelles et 8 RGT) en 4 jours par une seule personne (288 multiplex ; 992 fragments). De nouveau, toutes les mutations ont été détectées. Le design de cette étude a permis l'élaboration d'une bibliothèque de profils de référence des fragments mutés pour $B R C A I$, confirmant la reproductibilité de cette technique EMMA. Il est à noter que l'adaptabilité de la technologie EMMA a été facilitée par l'utilisation d'un kit commercial de réactifs de PCR multiplex. Les différentes études de validation menées à l'Institut Curie ou à l'Institut Bergonié sur $B R C A I$ démontrent la grande sensibilité, le haut débit et la facilité d'adaptation de la technologie EMMA. Au même titre que la dHPLC dans les années 97-98, la technologie EMMA pourrait devenir une méthode de référence de criblage des mutations géniques dans les laboratoires de diagnostic moléculaire.

Mots-clés : technique de criblage, diagnostic, $B R C A$.

\section{IP466 / 658. IDENTIFICATION D'UNE MUTATION STOP DANS LE GENE UPF3B ASSOCIÉE À UNE DÉFICIENCE MENTALE NON SPÉCIFIQUE LIÉE AU CHROMOSOME X}

F. Laumonnier (1), C. Cherpi-Antar (1), S. Blesson (2), C. Moraine (1, 2), M. Raynaud $(1,2)$, The European XLMR consortium

(1) INSERM U619, CHRU Bretonneau, Université François-Rabelais, Tours ; (2) Service de Génétique, CHRU Bretonneau, Tours

La déficience mentale (DM) constitue un problème majeur de santé publique car cette pathologie concerne 1 à $2 \%$ de la population générale. De récentes avancées ont permis de mieux comprendre les bases physiopathologiques associées aux formes génétiques de DM. Plus de 60 gènes de DM liée au chromosome X (DMX) ont maintenant été identifiés, et les protéines en cause sont impliquées dans de très nombreux processus intracellulaires différents. Parmi les gènes récemment caractérisés dans les $\mathrm{DMX}$, le gène $U P F 3 B$, localisé en Xq25, a été impliqué dans 4 familles, sur les 250 familles testées par une équipe anglaise (Tarpey et al., 2007). Trois mutations tronquantes différentes ont été identifiées dans 2 familles de syndrome de Lujan-Fryns et 1 famille de syndrome FG, une mutation faux-sens a été mise en évidence dans une famille de DMX non spécifique. Des troubles autistiques étaient associés chez certains patients. Le gène 
$U P F 3 B$, qui code pour une protéine impliquée dans le complexe intervenant dans la dégradation des ARNs comportant un codon stop prématuré (Nonsense Mediated Decay, NMD), semble avoir ainsi un impact majeur dans les fonctions cognitives. Afin d'évaluer l'implication plus large d'UPF3B dans les DMX, des mutations de ce gène ont été recherchées au sein de la cohorte de familles collectée par le « European XLMR consortium ". Nous avons sélectionné les familles du consortium pour lesquelles le territoire de liaison génétique contenait la région $\mathrm{Xq} 25$, soit 32 familles. Il s'agissait à la fois de familles de DM syndromique et de familles de DM non spécifique. L'amplification par PCR et le séquençage systématique de chaque exon codant du gène UPF3B ont été effectués. Dans le cas de l'identification d'une mutation, une RT-PCR quantitative est pratiquée afin de vérifier l'impact potentiel sur l'expression du transcrit. Dans une famille française comprenant 9 garçons atteints de DM légère à moyenne sans autre signe associé, nous avons pu identifier une mutation (c. $1083 \mathrm{C}>\mathrm{T}$ ) localisée dans le $10^{\circ}$ exon qui aboutit à la formation d'un codon stop prématuré en position 361 (Arg361X), alors que la protéine normale comporte 483 acides aminés. L'analyse de l'ADN de 48 membres de cette famille indique que cette mutation co-ségrège avec la pathologie dans la famille. Le profil d'inactivation du chromosome $\mathrm{X}$ chez les femmes conductrices indique une déviation modérée à complète, de la même manière que dans les familles décrites par Tarpey et al. Afin de vérifier le caractère délétère de la mutation, des expériences de RT-PCR quantitative et de Western-blots sont en cours. De façon intéressante, des observations à partir d'analyses en SiRNA ont montré qu'UPF3B régulait l'expression de protéines postsynaptiques comme GRIK2 et SHANK3, elles-mêmes impliquées dans la DM et l'autisme (Chan et al., 2007). Notre étude, basée sur l'analyse de 32 familles, a confirmé l'implication de ce gène dans les DMX et met en évidence une mutation tronquante dans une famille de DM non spécifique. La poursuite de l'analyse de toutes les familles du consortium (350) permettra d'estimer l'importance de ce gène dans les causes des DMX. Tarpey et al. (2007) Nat Genet 39, 1127-1133. Chan WK et al. (2007) EMBO J 26, 1820-1830.

Mots-clés : déficience mentale, chromosome X, perte de fonction.

P467 / 670. MUTATIONS DU GÈNE DU RÉCEPTEUR DE LA PROKINETICINE (PROKR2) DANS L'ANOSMIE CONGÉNITALE ISOLÉE

L. Tavernier (1), L. Villoing (2), P. Bonfils (3), N. de Roux $(2,4)$

(1) Service ORL, CHU de Besançon: (2) Laboratoire de Biochimie et Hormonologie, Hôpital Robert-Debré, Paris ; (3) Service ORL. HEGP. Paris ; (4) INSERM U690, Hôpital Robert-Debré, Paris

L'anosmie congénitale est définie par l'absence de tout souvenir olfactif dont la plus connue est celle du syndrome de Kallmann qui associe déficit gonadotrope et anosmie. Le lien physiopathologique entre déficit gonadotrope et anosmie est expliqué par l'agénésie des bulbes olfactifs observée dans ce syndrome, agénésie responsable de la perte de l'odorat et du défaut de migration des neurones a GnRH de la placode olfactive vers l'hypothalamus. Des mutations perte de fonction de plusieurs gènes (FGFRI, ProK2 et ProKR2) sont maintenant décrites dans le syndrome de Kallmann avec transmission autosomale dominante alors que les cas sporadiques d'anosmie congénitale isolée avec agénésie du bulbe olfactif sans défici gonadotrope reste inexpliquée. Nous avons testé l'hypothèse que des mutations des gènes FGFR1, ProKR2 et ProK2 pourraient être responsables de cette forme d'anosmie congénitale. 16 cas d'anosmie congénitale diagnostiquée chez des adultes ont été inclus dans cette étude. Aucun patient n'avait connaissance de retard pubertaire ou déficit gonadotrope dans sa famille. Le bilan hormonal était normal chez tous les patients. Les exons des gènes FGFR1, ProK2 et ProkR2 ont été séquencés et comparés à une population témoin. Aucune mutation n'a été trouvée dans le gène FGFRI. Deux mutations ponctuelles homozygotes de ProKR2 ont été trouvées chez deux patients. L'analyse familiale suggère une transmission récessive. ProkR2 est le récepteur de la prokineticine. Il appartient à la super famille des récepteurs couplés aux protéines $\mathrm{G}$. L'analyse fonctionnelle des mutations est en cours. Ces résultats indiquent qu'il faut analyser les gènes du syndrome de Kallmann en cas d'anosmie sporadique isolée sans déficit gonadotrope connu chez le patient ou dans sa famille. Ils suggèrent de nouvelles hypothèses sur les mécanismes de migration des neurones à GnRH durant l'embryogénèse.

Mots-clés : anosmie, ProKR2, neurones GnRH

IP468 / 673. CARACTÉRISATION FONCTIONNELLE DE TROIS NOUVELLES MUTATIONS FAUX-SENS DU GENE SLC40A1 (HÉMOCHROMATOSE DE TYPE 4)

E. Létocart (1), C. Ka (1), G. Isabelle (1), H. Fierens (2), W. Wuyts (2), S. Majore (3), G. Le Gac (1), C. Férec (1)
(1) Inserm, U613, France ; (2) Hôpital universitaire, Antwerp, Belgique ; (3) Hôpital Camillo-Forlanini, Rome, Italie

Introduction: La connaissance des bases génétiques et physiopathologiques de l'hémochromatose (HC) a fortement évoluée au cours des sept dernières années. Au-delà de la description d'une hétérogénéité génétique, qui associe une forme principale liée au gène $H F E$ ( $\mathrm{HC}$ de type 1) et quatre formes rares liées aux gènes HJV ( $\mathrm{HC}$ de type $2 \mathrm{~A}$ ), HAMP (HC de type 2B), TFR2 (HC de type 3) et SLC4OAI (HC de type 4), on reconnaît aujourd'hui l'existence d'une convergence fonctionnelle. Celle-ci s'articule autour de la production hépatique de l'hepcidine et de la capacité de ce peptide circulant à limiter la quantité de fer plasmatique. Le gène SLC4OAl code la ferroportine, une protéine membranaire qui permet d'exporter le fer contenu dans les macrophages (fer issu du catabolisme des globules rouges), les entérocytes (du fer d'origine alimentaire) et les hépatocytes (fer mis en réserve) vers le compartiment sanguin. En cas d'excès de fer, l'activité de cette protéine est inhibée par l'hepcidine selon un mécanisme d'internalisation et de dégradation lysosomale. De façon remarquable, les mutations du gène $S L C 4 O A I$ permettent de définir deux classes fonctionnelles. Elles correspondent respectivement à une perte ou à un gain de fonction (resistance à l'hepcidine) et permettent de rendre compte d'une part importante de la variabilité phénotypique observée. But de l'étude : Il s'agit de démontrer l'association de trois nouvelles variations du gène SLC4OAl avec des phénotypes de surcharge en fer au travers d'analyses fonctionnelles. Patients et méthodes: Les trois variations analysées sont privées. Elles ont respectivement été identifiées chez un patient belge, un patient italien et une patiente originaire de Côte d'Ivoire. Les tests fonctionnels ont porté sur des techniques de localisation cellulaire (par fragmentations membranaires et western-blottings) et de mesure des flux de fer en présence et en absence d'hépcidine (de manière directe, en utilisant du $55 \mathrm{Fe}$, et indirecte, en dosant les quantités de ferritine intracellulaire). Résultats: Les premières expériences réalisées permettent de conclure que les trois protéines mutantes testées sont correctement adressées à la membrane plasmique et qu'elles sont capables d'exporter le fer intracellulaire de façon comparable à la protéine de type sauvage. Nous testons actuellement la proportion de ces protéines à résister à l'action de l'hepcidine. Conclusion: Toutes les mutations décrites sur le gène SLC4OAI sont rares ou privées et une majorité de ces mutations conduisent à des changements d'acides aminés. En developpant des tests fonctionnels notre objectif à court terme était de démontrer la causalité de trois variations originales. À plus long terme, d'autres variations seront analysées et de nouveaux tests seront mis en cuvre. L'ensemble pourrait apporter de nouveaux éclairages sur la relation structure/fonction qui implique l'hepcidine et la ferroportine et qui constitue un élément clé dans le maintien de l'homéostasie du fer.

Mots-clés : hemochromatose, ferroportine, hepcidine.

E469 / 678. OPTIMISATION DES CONDITIONS PRÉ-ANALYTIQUES ET ANALYTIQUES POUR LA DÉTERMINATION PAR PCR QUANTITATIVE DU GÉNOTYPE RHÉSUS FETAL SUR L'ADN CIRCULANT DANS LE SANG MATERNEL

C. Petit (1), F.Gehin (1), S. Cristinelli (2), L. Zimbris (2), V. Petit (1) (1) Laboratoire LEFAURE \& PETIT, Epinal ; (2) Maternité Arc-en-Ciel, Épinal

Introduction: La prévention systématique de la maladie hémolytique du nouveau-né propose désormais, pour toute femme enceinte rhésus négatif, une injection systématique d'immunoglobuline anti-D au $8^{\circ}$ mois. Dans cette stratégie, la détection du génotype rhésus fơtal par amplification du gène $R H D$ à partir de l'ADN fætal circulant dans le sang maternel peut permettre d'éviter une injection qui s'avère en fait inutile si le fœetus est rhésus négatif, soit $40 \%$ des cas. Si la plupart des études converge maintenant en faveur d'une amplification des exons 4, 5, 7 et 10 du gène $R H D$ afin de garantir une sensibilité de détection de $100 \%$, plusieurs études ont également cherché à limiter la lyse des cellules maternelles non seulement par un protocole de centrifugation progressive mais aussi par l'ajout de formaldéhyde. L'ajout de formaldéhyde pourrait en effet, en fixant les cellules sanguines maternelles, éviter la rupture des membranes plasmiques avant et pendant la centrifugation, améliorant ainsi le pourcentage d'ADN fæetal jusqu'à $50 \%$. Nous avons cherché dans cette étude à optimiser les conditions préanalytiques permettant de limiter les contaminations maternelles: outre l'intérêt de l'ajout du formaldéhyde qui nécessitait d'être confirmé, nous avons évalué les possibilités de limiter la lyse des éventuelles cellules maternelles résiduelles dans le surnageant après la centrifugation progressive en éliminant l'étape de digestion enzymatique par la protéinase K lors l'extraction de l'ADN. Matériel et méthode : L'optimisation de l'extraction d'ADN fotal a été réalisée par amplification des gènes $S R Y$ et $B G L O B$ sur du sang de 58 femmes enceintes de garçon 
prélevé juste avant la naissance avec consentement de la patiente (Âge gestationnel moyen : $39,8 \mathrm{~s}$ ). Après centrifugation progressive, l'extraction d'ADN a été réalisée à partir de $400 \mu \mathrm{L}$ de plasma maternel sur colonne QIAamp ${ }^{\circ}$ DNA Mini (Quiagen). Le protocole de PCR quantitative du gène RHD a été évalué à partir de l'ADN foetal circulant dans le sang de 49 patientes enceintes Rh-négatif à différentes semaines d'aménorrhée avec une amplification à l'aide de sondes Taqman ${ }^{*}$ de quatre exons du gène RHD: l'exon 4, 5, 7 et 10. La corrélation des résultats des génotypes rhésus fœtaux a pu être vérifiée avec le phénotype rhésus déterminé lors du groupe sanguin effectué à la naissance au laboratoire. (Âge gestationnel moyen : $30,2 \mathrm{~s}$ ). Résultats : Le nombre de copies du gène de la $\beta$-globuline est clairement diminué par l'ajout d'une solution de formaldéhyde $0.1 \%$ avec une diminution de près de $15 \%$ du taux de contamination (B-GLOB/ $\mathrm{SRY}$ ). En revanche, l'élimination de la protéase $\mathrm{K}$ apporte certes une diminution des copies de BGLOB, mais le nombre de copies du gène fotal détecté diminue également fortement (chute de sensibilité de $40 \%$ ). Contrairement aux exons 7 et 10 parfaitement spécifiques, une amplification des exons 4 et 5 est observée respectivement dans $88 \%$ et $92 \%$ des patients Rh négatif du fait de l'importante homologie entre le gène $R H C E$ et $R H D$. En revanche, la sensibilité respective des exons 7 et 10 de $83,7 \%$ et $73,5 \%$ impose une amplification conjointe de ces deux exons pour éviter tout faux négatif. Le génotype fœtal a ainsi pu être déterminé pour les 49 femmes testées, jusqu'à un âge gestationnel minimum observé de 12,5 semaines, avec une corrélation de $100 \%$ au phénotype à la naissance. Conclusion : Notre étude confirme l'intérêt de l'ajout de formaldéhyde dès le prélèvement sanguin. En revanche, l'élimination de la digestion à la PK lors de l'extraction diminue certes la contamination maternelle mais diminue également l'accessibilité de l'ADN fotal pour l'amplification. $\mathrm{Si}$ les deux exons 4 et 5 ne présentent pas d'intérêt pour le génotypage Rh D en raison de leur manque de spécificité, la sensibilité des exons 7 et 10 n'est pas de $100 \%$ et leur amplification concomitante apparaît indispensable, avec en outre une vérification à distance en cas de résultat négatif.

Mots-clés : rhésus, ADN fœtal, PCR.

E470 I, 689. LA GÉNÉTIQUE DE L'ALBINISME OCULOCUTANÉ : RÉSULTATS MOLECULAIRES DANS UNE COHORTE EUROPÉENNE ET AFRICAINE

C. Rooryck (1, 2), F. Morice (2, 3), S. Naudion (2), V. Bubien (2), C. Plaisant (2), D. Lacombe (1, 2), A. Taieb (3), B. Arveiler (1, 2)

(1) Laboratoire de Génétique Humaine, Université Victor-Segalen, Bordeaux-2, Bordeaux; (2) Service de Génétique Médicale, Hôpital Universitaire Pellegrin-Enfants, Bordeaux; (3) Service de Dermatologie Pédiatrique, Hôpital Universitaire Pellegrin-Enfants, Bordeaux

Introduction : L'albinisme oculo-cutané (AOC) est une maladie de transmission autosomique récessive caractérisée par une hypopigmentation de la peau, des phanères, et des yeux (iris, rétine). Elle entraîne des troubles ophtalmologiques à type de nystagmus, baisse d'acuité visuelle, photophobie, strabisme, et une prédisposition de la peau aux cancers cutanés. Sa prévalence générale mondiale est de 1/20 000. Quatre gènes sont impliqués dans les quatre types connus d'AOC non syndromique: TYR en $11 \mathrm{q} 14.3$ dans I'AOC1, OCA2 en $15 \mathrm{q} 11.2$ dans 1'AOC2, TYRPI en 9p23 dans l'AOC3, et SLC45A2 (MATP) en 5p13.3 dans l'AOC4. Matériel et Méthodes: Nous avons étudié une cohorte de 62 patients atteints de différents types d'AOC et provenant de plusieurs pays européens et africains. L'analyse moléculaire des quatre gènes de l'AOC comprend la recherche de délétion intragénique par PCR semi-quantitative (QMF-PCR) et la recherche de mutation ponctuelle par séquençage direct (TYR) ou par DHPLC suivie du séquençage des variants (OCA2, TYRPI, SLC45A2). Résultats: Nous avons identifié de nouvelles mutations dans les quatre gènes : 8 mutations ponctuelles dans TYR, 10 dans $O C A 2,4$ dans TYRPI et 4 dans $S L C 45 A 2$. Des délétions ont également été identifiées : une délétion hétérozygote englobant tout le gène $T Y R$, de nouvelles délétions intragéniques homozygotes ou hétérozygotes englobant un ou plusieurs exons du gène $O C A 2$, une délétion hétérozygote d'un exon du gène MATP. Une délétion comprenant $O C A 2$ et $U B E 3 A$ a été identifiée chez un patient turc présentant un AOC et un syndrome d'Angelman. Nous avons détecté deux mutations de TYRPI chez un patient Caucasien, alors que des mutations de ce gène étaient jusqu'alors seulement identifiées chez des Africains. Discussion : Cette étude nous a permis de réévaluer la fréquence mondiale des mutations pour chaque gène d'AOC. Parmi les allèles mutés, les mutations dans TYR étaient prédominantes ( $44 \%)$, puis OCA2 $(38 \%)$ MATP (13\%) et TYRPl $(5 \%)$. L'étude systématique de MATP a permis de mettre en évidence une prévalence plus élevée des mutations dans ce gène par rapport aux données actuelles de la littérature. $70 \%$ de patients sont homozygotes ou hétérozygotes composites, $24 \%$ sont porteurs d'une seule muta- deux hypothèses : soit les mutations non identifiées sont dans des régions encore non explorées des gènes (tels que les introns ou les éléments régulateurs) soit il existe d'autres gènes d'AOC non connus à ce jour. Enfin, deux patients présentant trois mutations dans deux gènes différents nous font suggérer une possible hérédité triallélique.

Mots-clés : albinisme oculocutané, tyrosinase, hypopigmentation.

\section{Modèles cellulaires et animaux}

EP471 / 244. UTILISATION DU MODÈLE CANIN POUR RECHERCHER DE NOUVEAUX GÊNES IMPLIQUÉS DANS L'ÉPILEPSIE

A. Grall (1), L. Herbin (1), B. Hédan (1), M. Beldame (1), C. Hitte (1), A. Thomas (2), C. Escriou (3), F. Galibert (1), C. André (1)

(1) Laboratoire de Génétique et Développement, UMR 6061 CNRS/Université de Rennes1; (2) Antagene, Laboratoire de Génétique Animale, Limonest; (3) Unité de Médecine, Neurologie, École Nationale Vétérinaire de Lyon

Le chien, par le grand nombre de races distinctes se comportant chacune comme un isolat génétique, représente un puissant modèle pour identifier les gènes et les allèles responsables d'affections génétiques homologues à celles de l'homme. En effet, la plupart des maladies génétiques canines ont leur équivalent chez l'homme, elles sont spécifiques de race et ségrégent avec une incidence élevée. L'épilepsie représente le désordre neurologique le plus répandu dans l'espèce canine avec $5 \%$ de chiens atteints, répartis dans plus de cinquante races. La forte prévalence de l'épilepsie dans certaines races, atteignant des fréquences supérieures à $20 \%$, laisse supposer une origine génétique. L'hypothèse est que chaque race ou petit groupe de races développe une forme d'épilepsie liée à une cause génétique spécifique provenant d'une mutation fondatrice au moment de la création de la race. Ainsi, l'étude des bases moléculaires de l'épilepsie dans ces différentes races canines permettrait d'enrichir le nombre de gènes candidats pour des épilepsies humaines dont les bases génétiques sont encore inconnues. La découverte récente de l'implication du gène $E P M 2 B$ dans la maladie de Lafora chez l'homme (forme d'épilepsie myoclonique progressive) et dans l'épilepsie affectant le Teckel miniature à poil dur démontre l'intérêt de ce modèle. Dans ce but, nous collectons l'ADN de chiens épileptiques de plusieurs races et avons constitué une famille de plus de 100 Grands Bouviers Suisses comportant 11 chiens atteints d'une forme d'épilepsie primaire de type " grand mal » à déclaration précoce (entre 2 et 4 ans). La physiopathologie et l'épidémiologie de cette affection chez le Grand Bouvier Suisse est en cours d'étude, ainsi que le mode de ségrégation qui semble monogénique récessif. Disposer d'une grande famille avec des fratries complètes comportant des chiens sains et atteints a permis d'entreprendre une analyse de liaison génétique avec 300 marqueurs microsatellites polymorphes, répartis selon un intervalle de $10 \mathrm{Mb}$ sur la totalité des chromosomes canins. En parallèle, les gènes déjà identifiés dans différentes épilepsies chez l'homme ont été répertoriés de façon à corréler, le cas échéant, leur localisation avec le ou les locus candidats de cette étude. En complément, un criblage génomique d'une cinquantaine de chiens de cette famille est en projet, grâce à l'utilisation de puces SNP permettant simultanément l'analyse allélique de 44000 marqueurs canins de type SNP (Single Nucleotide Polymorphism). Ces deux approches complémentaires permettront de déterminer, puis de réduire significativement le locus candidat jusqu'à l'identification du gène et ou de la mutation causale.

Mots-clés : modèle canin, liaison génétique, épilepsie.

\section{IP472 / 314. EXPLORATION DU MODÈLE MURIN KNOCK-IN LMNA R298C/R298C, ET PERSPECTIVES SUR LA PRÉSENCE D'UN DOMAINE LEUCINE-ZIPPER DANS LES LAMINES}

Y. Poitelon (1), T. Hamadouche (1), C. Baubot (1), M. Jamon (1), V. Andrés (2), N. Levy $(1,3)$, V. Delague (1)

(1) UMR910, Génétique Médicale et Génomique Fonctionnelle, Faculté de Médecine de la Timone, Marseille, France ; (2) Laboratory of Vascular Biology, Instituto de Biomedicina de Valencia, Valencia, Spain; (3) Département de Génétique Médicale, Hôpital d'enfants de la Timone, Marseille, France

Le gène $L M N A$ code pour les Lamines de type $\mathrm{A}$, dont les deux isoformes majeures sont les Lamines A et C. Ces protéines ubiquitaires sont les principaux constituants de la lamina nucléaire, réseau filamenteux accolé à la face interne de la membrane nucléaire. Onze pathologies ont été associées à des mutations du gène $L M N A$. Elles sont regroupées sous le terme de laminopathies, et peuvent être des affections systémiques ou bien spécifiques d'un seul tissu. La maladie de Charcot-Marie-Tooth de type 2B1, 